\title{
Drinfeld Realization of Affine Quantum Algebras: the Relations
}

\author{
by
}

Ilaria DAMIANI

\author{
To women. \\ Especially to those who do not have \\ even the opportunity to imagine \\ how much they would like mathematics \\ and to those who are forced to forget it
}

\begin{abstract}
The structure of the Drinfeld realization $\mathcal{U}_{q}^{\mathrm{Dr}}$ of affine quantum algebras (both untwisted and twisted) is described in detail, and its defining relations are studied and simplified. As an application, a homomorphism $\psi$ from this realization to the Drinfeld and Jimbo presentation $\mathcal{U}_{q}^{\mathrm{DJ}}$ is provided, and proved to be surjective.
\end{abstract}

2010 Mathematics Subject Classification: Primary 17B37.

Keywords: quantum groups.

\section{$\S 0$. Introduction}

Let $X_{\tilde{n}}^{(k)}$ be a Dynkin diagram of affine type, $\mathcal{U}_{q}^{\mathrm{DJ}}=\mathcal{U}_{q}^{\mathrm{DJ}}\left(X_{\tilde{n}}^{(k)}\right)$ the quantum algebra introduced by Drinfeld and Jimbo (see [Dr2] and [Jm]), and $\mathcal{U}_{q}^{\operatorname{Dr}}=\mathcal{U}_{q}^{\operatorname{Dr}}\left(X_{\tilde{n}}^{(k)}\right)$ its Drinfeld realization (see [Dr1]).

This paper has two main goals: describing in detail the structure of the Drinfeld realization $\mathcal{U}_{q}^{\mathrm{Dr}}$ with sharply simplified defining relations; and constructing a (surjective) homomorphism $\psi$ from this realization to the Drinfeld and Jimbo presentation $\mathcal{U}_{q}^{\mathrm{DJ}}$, as a step towards a complete proof that $\mathcal{U}_{q}^{\mathrm{DJ}}$ and $\mathcal{U}_{q}^{\mathrm{Dr}}$ are isomorphic, so that they are indeed different presentations of the same $\mathbb{C}(q)$-algebra $\mathcal{U}_{q}=\mathcal{U}_{q}\left(X_{\tilde{n}}^{(k)}\right)($ see $[\operatorname{Dr} 1])$.

Communicated by H. Nakajima. Received June 25, 2011. Revised November 17, 2011.

I. Damiani: Department of Mathematics, University of Rome "Tor Vergata", 00133 Roma, Italy; e-mail: damiani@mat.uniroma2.it

(C) 2012 Research Institute for Mathematical Sciences, Kyoto University. All rights reserved. 
Understanding the isomorphism between $\mathcal{U}_{q}^{\mathrm{DJ}}$ and $\mathcal{U}_{q}^{\mathrm{Dr}}$ stated by Drinfeld in [Dr1] has important applications in the study of the representation theory of affine quantum algebras: using this result, the finite-dimensional irreducible representations of affine quantum algebras are classified in [CP1], [CP2] and [CP3]; and a geometrical realization (through the quiver varieties) of finite-dimensional representations is constructed in $[\mathrm{N}]$ for the untwisted simply laced cases.

The interest of the twisted case resides not only in that it is a generalization of the untwisted frame. Actually twisted algebras appear quite naturally while studying the untwisted setting, due to the fact that transposition of matrices establishes a duality among the affine Cartan matrices through which untwisted Cartan matrices can correspond to twisted ones; more precisely simply laced untwisted matrices and matrices of type $A_{2 n}^{(2)}$ are self-dual, while transposition operates on the remaining affine Cartan matrices by interchanging untwisted and twisted ones. This observation is important and concrete because of results like those in [CP4], where the quantum symmetry group of the affine Toda field theory associated to an untwisted affine Kac-Moody algebra is proved to be the quantum algebra associated to the dual Kac-Moody algebra; and in [FH], where the authors conjecture in general, and prove for the Kirillov-Reshetikhin modules, that there exists a duality between representations of an untwisted affine quantum algebra and those of the dual quantum algebra.

Much work has already been done in the direction of understanding Drinfeld's theorem. In [Be] all the relations are proved in the untwisted case. Notice that this does not yet imply that $\psi$ is an isomorphism: indeed, the argument for the injectivity should be completed with the proof of the existence of a basis of the integer form, necessary to conclude that the injectivity at 1 implies the injectivity at level $q$; this point is not discussed and as far as I understand it is non-trivial.

For the twisted case there are several partial results. In [A] the author studies case $A_{2}^{(2)}$, constructing $\psi$ following [Be], but the proof that it is well defined is incomplete; a contribution to this proof is given in $[\mathrm{H}]$.

In [Jn], [JZ2] and [JZ1], the authors construct a homomorphism from $\mathcal{U}_{q}^{\mathrm{DJ}}\left(X_{\tilde{n}}^{(k)}\right)$ to $\mathcal{U}_{q}^{\mathrm{Dr}}\left(X_{\tilde{n}}^{(k)}\right)$ (the inverse of $\psi$ ) following the theorem stated by Drinfeld in [Dr1], that is, by means of $q$-commutators. In [Jn] the author gives some details in the untwisted case, sketching the proof of the relations $\left[E_{0}, F_{i}\right]=0$ $\left(i \in I_{0}\right)$ in case $A_{3}^{(1)}$, of the Serre relation $E_{0} E_{1}^{2}-\left(q+q^{-1}\right) E_{1} E_{0} E_{1}+E_{1}^{2} E_{0}=0$ in case $A_{n}^{(1)}$ (noticing that the Serre relations involving just indices in $I_{0}$ are trivial, but the other Serre relations involving $E_{0}$ are not studied, for instance $E_{1} E_{0}^{2}-\left(q+q^{-1}\right) E_{0} E_{1} E_{0}+E_{0}^{2} E_{1}=0$ is missing $)$ and of the relations $\left[E_{0}, F_{0}\right]=$ $\frac{K_{0}-K_{0}^{-1}}{q_{0}-q_{0}^{-1}}$ in cases $A_{n}^{(1)}$ and $C_{2}^{(1)}$; but a strategy for generalizing these arguments 
is not presented, and the twisted case is just stated to be similar. In [JZ2] the authors concentrate on the twisted case, but their work is again incomplete since the Serre relations involving indices $i \neq j \in I_{0}$ are treated, erroneously, as in the untwisted case, and for the other relations the authors present some examples: the commutation between $E_{0}$ and $F_{i}(i \in I)$ is studied in cases $A_{2}^{(2)}$ and $D_{4}^{(3)}$; some Serre relations (but not all of them) involving $E_{0}$ are studied in cases $A_{2 n-1}^{(2)}$ and $D_{4}^{(3)}$; and again a strategy for generalizing these computations is not shown. Also in [JZ2] there is a mistake in the connection between the data of a finite Dynkin diagram and its non-trivial automorphism on one hand and the twisted affine Dynkin diagram on the other hand, which has consequences in the following paper [JZ1]. Finally in [JZ1] the authors want to fill the gap about the Serre relations involving the indices $i, j \in I_{0}$ such that $a_{i j}<-1$ (in the twisted case), and they use a case by case approach; but the Drinfeld relations are misunderstood, and stated to imply relations not holding in this algebra.

These difficulties suggest the need to better understand the Drinfeld realization, which is the aim of the present paper; the definition of the homomorphism $\psi$ from the Drinfeld realization to the Drinfeld and Jimbo presentation of affine quantum algebras then becomes a simple consequence of this analysis, and it is also proved that $\psi$ is surjective.

In $\S 1$ and $\S 2$ we recall the notions of Dynkin diagram, Weyl group and root system, and their properties needed in the arguments of the following sections; in particular it is recalled how untwisted and twisted affine Dynkin diagrams, Weyl groups and root systems are connected to finite ones, together with their classification and basic properties.

In $\S 3$ some preliminary material about the presentation $\mathcal{U}_{q}^{\mathrm{DJ}}$ of Drinfeld and Jimbo of the affine quantum algebras is summarized.

In Definitions 3.2 and 3.3 and in Remark 3.4 we recall the definition of $\mathcal{U}_{q}^{\mathrm{DJ}}$, its main structures ( $Q$-gradation, triangular decomposition, antiautomorphisms $\Omega$ and $\Xi$, braid group action, embedding of the finite quantum algebra in the affine one, root vectors $E_{\alpha}$ ) and properties (commutation of (anti)automorphisms, connection between the braid group action and root vectors, Poincaré-BirkhoffWitt basis, Levendorskii-Soibelman formula).

We also recall the embeddings $\varphi_{i}$ of the rank 1 quantum algebras $\mathcal{U}_{q}^{\mathrm{DJ}}\left(A_{1}^{(1)}\right)$ and $\mathcal{U}_{q}^{\mathrm{DJ}}\left(A_{2}^{(2)}\right)$ in the general quantum algebra $\mathcal{U}_{q}^{\mathrm{DJ}}\left(X_{\tilde{n}}^{(k)}\right)$ and their properties of commutation and injectivity (Definition 3.6 and Remark 3.7). They will play a role in the comparison between the Drinfeld realization and the Drinfeld-Jimbo presentation in $\S 12$ (Theorem 12.7). 
In $\S 4$ we give the definition of the Drinfeld realization of affine quantum algebras (both untwisted and twisted, see [Dr1]), discussing and translating the relations into a more explicit form, easier for the purpose of this paper. Even if it is just a reformulation, it seems useful to give the details, since they are not always clear in the literature.

In $\S 5$ some notation is fixed in order to simplify the analysis of the relations. Also some relations are reformulated in terms of $q$-commutators, and some new relations, including the Serre relations $\left(S^{ \pm}\right)$and other similar ones $\left(\left(T 2^{ \pm}\right)\right.$and $\left.\left(T 3^{ \pm}\right)\right)$, are introduced, which will play an important role in $\S 10$ and $\S 11$.

In $\S 6$ the main structures on $\mathcal{U}_{q}^{\mathrm{Dr}}$ are introduced: the $Q$-gradation; the homomorphisms $\phi_{i}$, underlining the role of the two affine Drinfeld realizations of rank one, $A_{1}^{(1)}$ and $A_{2}^{(2)}$, which embed in any other Drinfeld realization, each embedding depending on the choice of a vertex of the ("finite part" of the) Dynkin diagram; the antiautomorphism $\Omega$, describing the correspondence between "positive" and "negative" vectors $X_{i, r}^{ \pm}$; the automorphisms $\Theta$ and $t_{i}$ (for each $i \in I_{0}$ ), which summarize several symmetries (reflection about zero and translations) among the "positive" vectors. Actually these structures are defined on the algebra $\overline{\mathcal{U}}_{q}^{\text {Dr }}$ (which is also defined in this section), of which the Drinfeld realization is a quotient, and the proof that they induce analogous structures on $\mathcal{U}_{q}^{\mathrm{Dr}}$ is quickly concluded in $\S 8$, through the discussion of $\S 7$.

In $\S 7$ the algebra $\tilde{\mathcal{U}}_{q}^{\mathrm{Dr}}$, which is an algebra (already introduced in the previous section) intermediate between $\overline{\mathcal{U}}_{q}^{\mathrm{Dr}}$ and $\mathcal{U}_{q}^{\mathrm{Dr}}$, is studied in detail. In particular a first set of relations is simplified: the most important remarks are that the relations $\left(H X^{ \pm}\right)$can be replaced by the much easier $\left(H X L^{ \pm}\right)$(see Proposition 7.15 ; they are much easier not only because they are a smaller set of relations, but mainly because they can be expressed just in terms of $q$-commutation of the generators $X_{i, r}^{ \pm}$of $\overline{\mathcal{U}}_{q}^{\text {Dr }}$, without using the $H_{i, r}$ 's, see Remark 7.18 ); and that the relations $(H H)$ are also redundant (see Proposition 7.16). But also the other relations are studied and interpreted while discussing how the structures on $\overline{\mathcal{U}}_{q}^{\mathrm{Dr}}$ (see $\S 6$ ) induce analogous structures on $\tilde{\mathcal{U}}_{q}^{\mathrm{Dr}}$ (see Remarks 7.7 and 7.9).

$\S 8$ is a short and simple section where the structures defined on $\overline{\mathcal{U}}_{q}^{\mathrm{Dr}}$ and induced on $\tilde{\mathcal{U}}_{q}^{\text {Dr }}$ are proved to pass also to $\mathcal{U}_{q}^{\text {Dr }}$; this simple analysis is carried out explicitly, fixing some notation, in order to use it in further considerations, especially in $\S 9$.

In $\S 9$ it is now possible to start concentrating on the simplification of the relations defining $\mathcal{U}_{q}^{\mathrm{Dr}}$ over $\tilde{\mathcal{U}}_{q}^{\text {Dr }}$; these are the relations involving just the $X_{i, r}^{+}$'s or just the $X_{i, r}^{-}$'s, and there is a correspondence between the two cases thanks to the action of $\tilde{\Omega}$. The main result of this section is that the dependence of these relations on parameters $\left(r_{1}, \ldots, r_{l}\right) \in \mathbb{Z}^{l}\left(l \in \mathbb{Z}_{+}\right)$is redundant: we can indeed just 
restrict to the same relations indexed by $(r, \ldots, r) \in \mathbb{Z}^{l}$ where $r \in \mathbb{Z}$ (the "constant parameter" relations), so that the dependence on $\mathbb{Z}^{l}$ is reduced to a dependence on an integer $r$ (see Lemmas 9.12 and 9.14, Proposition 9.15 and Corollary 9.19); on the other hand, thanks to the action of the $\tilde{t}_{i}$ 's, this situation can be again simplified by just analyzing the relations relative to $(0, \ldots, 0)$ (see Remark 9.8).

Thanks to the results of $\S 9$ the study of the relations defining $\mathcal{U}_{q}^{\mathrm{Dr}}$ can be pushed forward: in $\S 10$ further dependences among the relations are proved (Propositions 10.1 and 10.4, Corollary 10.6 and Remark 10.7). These results are summarized in Theorem 10.8 and in Corollary 10.9, where a "minimal" set of relations is provided.

The last step of this analysis is the study of the Serre relations, performed in $\S 11$; here the relations $\left(X D^{ \pm}\right)-\left(S 3^{ \pm}\right)$are proved to depend, in the case of rank greater than 1, on the ("constant parameter") Serre relations, and these are vice versa proved to depend on the relations $\left(X D^{ \pm}\right)-\left(S 3^{ \pm}\right)$also in the cases in which this is not tautologically evident $\left(k>1, a_{i j}<-1\right)$. Theorem 11.18 and Corollary 11.19 state the final result of this study, and are the main tool for constructing the homomorphism $\psi$ and for proving that it is well defined (see $\S 12$ ).

$\S 12$ is devoted to constructing a homomorphism $\psi$ from $\mathcal{U}_{q}^{\mathrm{Dr}}$ to $\mathcal{U}_{q}^{\mathrm{DJ}}$ and to proving that it is well defined and surjective.

In Definition 12.3, $\tilde{\psi}: \tilde{\mathcal{U}}_{q}^{\mathrm{Dr}} \rightarrow \mathcal{U}_{q}^{\mathrm{DJ}}$ is defined, following [Be]. It just requires some care in the determination of the sign $o$ (Notation 12.1 and Remark 12.2).

The results of $\S 11$ and the correspondence, described in Proposition 12.4, between the (anti)automorphisms constructed on $\mathcal{U}_{q}^{\mathrm{Dr}}$ and those already known on $\mathcal{U}_{q}^{\text {DJ }}$ make the goal of proving that $\tilde{\psi}$ induces $\psi$ on $\mathcal{U}_{q}^{\text {Dr }}$ trivial in the cases of rank greater than 1 , that is, in all cases different from $A_{1}^{(1)}$ and $A_{2}^{(2)}$ (Theorem 12.5).

We give two different arguments to solve the cases of rank one (Theorem 12.7). The first one is based on the direct computation of the simple commutation relation between $E_{1}$ and $E_{\delta+\alpha_{1}}$ in $\mathcal{U}_{q}^{\mathrm{DJ}}\left(A_{1}^{(1)}\right)$ and $\mathcal{U}_{q}^{\mathrm{DJ}}\left(A_{2}^{(2)}\right)$ (Lemma 12.6). The second one is a straightforward corollary of the result in the case of rank greater than 1, once one recalls the embeddings (see Remark 3.7) of rank 1 quantum algebras in general quantum algebras.

A proof that $\psi$ is surjective is provided in Theorem 12.11: it makes use of the correspondence between the automorphisms $t_{i}$ on $\mathcal{U}_{q}^{\mathrm{Dr}}$ and the automorphisms $T_{\lambda_{i}}$ on $\mathcal{U}_{q}^{\mathrm{DJ}}$ and among the $\Omega$ 's (Remark 12.8), and of the braid group action on $\mathcal{U}_{q}^{\mathrm{DJ}}$.

Theorem 12.11 would also suggest how to define the inverse of $\psi$.

An index of notation used in the paper is in the appendix (§13). 
I am deeply grateful to David Hernandez for proposing me to work again on the twisted affine quantum algebras: I abandoned them too many years ago, and would have neither planned nor dared to approach them again if he had not encouraged and motivated me.

I take this occasion to make explicit my gratitude to Corrado De Concini, my maestro: for his always caring presence (even when he did not approve my choices) in the vicissitudes of my relationship with mathematics, and for his belief (undeserved yet helpful) he made me always feel. Not accidentally, the idea of this work was born at a conference for his $60^{\text {th }}$ birthday.

To Andrea Maffei I owe much: because we have been sharing reflections and projects about mathematics and our work since we were students till our adult life; because he is a rare, precious intellectual; and because he is (and has been on this occasion) ready to listen to and help with big and small problems, however specific they can be. But I owe him even more: his always personal points of view and his friendship.

Eleonora Ciriza is for me more than a colleague, than a mathematician, than an unreplaceable friend: she is all this together. Her support and advice are deeprooted in a way of being in the world that opened my mind and my life beyond the borders of my own experience.

I do not know if I would have ever arrived at the end of this paper without Salvatore, who had the difficult role of indicating me the purpose of concluding this work as a priority. In particular during the drawing up of the paper, he had to fight hard against my resistance to cut the myriad of other "priorities" which absorb much of my concentration and time, and against my delaying attitude of, like Penelope, always undoing what I have done. I thank him for believing in the importance of my work in my and our life.

\section{$\S 1$ Preliminaries: Dynkin diagrams}

For the preliminary material in this section see $[\mathrm{Bo}]$ and $[\mathrm{K}]$.

A Dynkin diagram $\Gamma$ of finite or affine type is the datum $(I, A)$ of its set of indices $I$ and its Cartan matrix $A=\left(a_{i j}\right)_{i, j \in I} \in \mathcal{M}_{n \times n}(\mathbb{Z})$ with the following properties:

(i) $a_{i i}=2$ for all $i \in I$;

(ii) $a_{i j} \leq 0$ for all $i \neq j \in I$;

(iii) $a_{i j}=0 \Leftrightarrow a_{j i}=0$;

(iv) the determinants of all the proper principal minors of $A$ are positive, and $\operatorname{det}(A) \geq 0$ ( $\Gamma$ is of finite type if $\operatorname{det}(A)>0$ and of affine type if $\operatorname{det}(A)=0)$; 
$\Gamma$ is said to be indecomposable if furthermore:

(v) if $I=I^{\prime} \cup I^{\prime \prime}$ with $I^{\prime} \cap I^{\prime \prime}=\emptyset$ and $I^{\prime}, I^{\prime \prime} \neq \emptyset$ then there exist $i^{\prime} \in I^{\prime}$ and $i^{\prime \prime} \in I^{\prime \prime}$ such that $a_{i^{\prime} i^{\prime \prime}} \neq 0$.

Between the vertices $i \neq j \in I$ there are $\max \left\{\left|a_{i j}\right|,\left|a_{j i}\right|\right\}$ edges, with an arrow pointing to $i$ if $\left|a_{i j}\right|>\left|a_{j i}\right|$; vertices, edges and arrows uniquely determine $\Gamma$.

A Dynkin diagram automorphism of $\Gamma$ is a map $\chi: I \rightarrow I$ such that $a_{\chi(i) \chi(j)}=a_{i j}$ for all $i, j \in I$.

It is universally known that these data are classified (see [Bo]); the type of the indecomposable finite data is denoted by $X_{\# I}(X=A, B, C, D, E, F, G)$.

In this preliminary section we recall the construction and classification of the indecomposable Dynkin diagrams of affine type due to Kac (see $[\mathrm{K}]$ ) and fix the general notation used in the paper.

Let $\tilde{\Gamma}$ be an indecomposable Dynkin diagram of finite type, with set of vertices $\tilde{I}(\# \tilde{I}=\tilde{n})$ and Cartan matrix $\tilde{A}=\left(\tilde{a}_{i^{\prime} j^{\prime}}\right)_{i^{\prime}, j^{\prime} \in \tilde{I}}$. To $X_{\tilde{n}}$ there are attached:

(a) the root lattice $\tilde{Q}=\bigoplus_{i^{\prime} \in \tilde{I}} \mathbb{Z} \tilde{\alpha}_{i^{\prime}}$;

(b) the Weyl group $\tilde{W} \subseteq \operatorname{Aut}(\tilde{Q})$ generated by the reflections $\left\{\tilde{s}_{i^{\prime}} \mid i^{\prime} \in \tilde{I}\right\}$ where $\tilde{s}_{i^{\prime}}$ is defined by $\tilde{s}_{i^{\prime}}\left(\tilde{\alpha}_{j^{\prime}}\right)=\tilde{\alpha}_{j^{\prime}}-\tilde{a}_{i^{\prime} j^{\prime}} \tilde{\alpha}_{i^{\prime}}\left(i^{\prime}, j^{\prime} \in \tilde{I}\right)$;

(c) the (uniquely determined up to a scalar factor) $\tilde{W}$-invariant bilinear form $(\cdot \mid \cdot)$ on $\tilde{Q}$, which induces a positive definite scalar product on $\mathbb{R} \otimes_{\mathbb{Z}} \tilde{Q}=\bigoplus_{i^{\prime} \in \tilde{I}} \mathbb{R} \tilde{\alpha}_{i^{\prime}}$;

(d) the root system $\tilde{\Phi} \subseteq \tilde{Q}$, which is the $\tilde{W}$-orbit of the set $\left\{\tilde{\alpha}_{i^{\prime}} \mid i^{\prime} \in \tilde{I}\right\}$ and is also characterized by $\tilde{\Phi}=\left\{\tilde{\alpha} \in \tilde{Q} \mid \exists i^{\prime} \in \tilde{I}\right.$ such that $\left.(\tilde{\alpha} \mid \tilde{\alpha})=\left(\tilde{\alpha}_{i^{\prime}} \mid \tilde{\alpha}_{i^{\prime}}\right)\right\}$.

A Dynkin diagram automorphism $\chi$ induces an orthogonal transformation $\chi$ of $(\tilde{Q},(\cdot \mid \cdot))\left(\chi\left(\tilde{\alpha}_{i^{\prime}}\right)=\tilde{\alpha}_{\chi\left(i^{\prime}\right)}\right)$, and we have $\chi \circ \tilde{s}_{i^{\prime}}=\tilde{s}_{\chi\left(i^{\prime}\right)} \circ \chi, \chi(\tilde{\Phi})=\tilde{\Phi}$.

Consider the datum $\left(X_{\tilde{n}}, \chi\right)$ with $\chi$ a Dynkin diagram automorphism of $X_{\tilde{n}}$, and let $k$ be the order of $\chi$. It is well known (see $[K]$ ) that to this datum it is possible to attach an indecomposable Dynkin diagram $\Gamma$ of affine type and an indecomposable subdiagram $\Gamma_{0} \hookrightarrow \Gamma$ of finite type with the following properties:

(I) the sets of vertices $I$ of $\Gamma$ and $I_{0}$ of $\Gamma_{0}$ are related by $I_{0}=\tilde{I} / \chi$ (the set of $\chi$-orbits in $\tilde{I}$; for $i^{\prime} \in \tilde{I}$ denote by $\overline{i^{\prime}} \in I_{0}$ the $\chi$-orbit of $\left.i^{\prime}\right)$ and $I=I_{0} \cup\{0\}$; we shall denote by $n$ the cardinality of $I_{0}$ and by $\{1, \ldots, n\}$ the set $I_{0}$ (so that $I=\{0,1, \ldots, n\})$;

(II) the Cartan matrix $A_{0}$ of $\Gamma_{0}$ is connected with $\tilde{A}$ through the relation

$$
a_{\overline{i^{\prime} j^{\prime}}}=2 \frac{\sum_{u \in \mathbb{Z} / k \mathbb{Z}} \tilde{a}_{\chi^{u}\left(i^{\prime}\right) j^{\prime}}}{\sum_{u \in \mathbb{Z} / k \mathbb{Z}} \tilde{a}_{\chi^{u}\left(i^{\prime}\right) i^{\prime}}} ;
$$

note in particular that if $k=1$ we have $I_{0}=\tilde{I}$ and $A_{0}=\tilde{A}$, hence $\Gamma_{0}=\tilde{\Gamma}$; 
(III) the root lattice $Q_{0}=\bigoplus_{i \in I_{0}} \mathbb{Z} \alpha_{i}$ of $\Gamma_{0}$ naturally embeds in the root lattice $Q=\bigoplus_{i \in I} \mathbb{Z} \alpha_{i}$ of $\Gamma$; their positive subsets are $Q_{0,+}=\sum_{i \in I_{0}} \mathbb{N} \alpha_{i}$ and $Q_{+}=\sum_{i \in I} \mathbb{N} \alpha_{i}$

(IV) the highest root $\vartheta_{0}$ of $\Gamma_{0}$ is characterized by the properties that $\vartheta_{0} \in \Phi_{0}$ (the root system of $\left.\Gamma_{0}\right)$ and $\vartheta_{0}-\alpha \in Q_{0,+}$ for all $\alpha \in \Phi_{0} ;$ moreover $\left(\vartheta_{0} \mid \vartheta_{0}\right) \geq(\alpha \mid \alpha)$ for all $\alpha \in \Phi_{0}$;

(V) the highest shortest root $\vartheta_{0}^{(s)}$ of $\Gamma_{0}$ is characterized by the properties that $\vartheta_{0}^{(s)} \in \Phi_{0},\left(\vartheta_{0}^{(s)} \mid \vartheta_{0}^{(s)}\right) \leq(\alpha \mid \alpha)$ for all $\alpha \in \Phi_{0}$, and $\vartheta_{0}^{(s)}-\alpha \in Q_{0,+}$ for all $\alpha \in \Phi_{0}$ such that $(\alpha \mid \alpha)=\left(\vartheta_{0}^{(s)} \mid \vartheta_{0}^{(s)}\right)$;

(VI) the Cartan matrix $A$ of $\Gamma$ extends $A_{0}: A=\left(a_{i j}\right)_{i, j \in I}$, with

$$
a_{00}=2, \quad \forall i \in I_{0}, \quad a_{0 i}=-2 \frac{\left(\theta \mid \alpha_{i}\right)}{(\theta \mid \theta)}, \quad a_{i 0}=-2 \frac{\left(\alpha_{i} \mid \theta\right)}{\left(\alpha_{i} \mid \alpha_{i}\right)},
$$

where

$$
\theta= \begin{cases}\vartheta_{0} & \text { if } k=1 \\ 2 \vartheta_{0}^{(s)} & \text { if } X_{\tilde{n}}=A_{2 n} \text { and } \chi \neq \mathrm{id} \\ \vartheta_{0}^{(s)} & \text { otherwise. }\end{cases}
$$

The type of the Dynkin diagram $\Gamma$ thus constructed is denoted by $X_{\tilde{n}}^{(k)}$ (indeed it does not depend on $\chi$ but just on $k$ ), and it is well known (see $[\mathrm{K}]$ ) that this construction provides a classification of the indecomposable affine Dynkin diagrams, which we list in the following table.

The labels under the vertices fix an identification between $I$ and $\{0,1, \ldots, n\}$ such that $I_{0}$ corresponds to $\{1, \ldots, n\}$. For each type we also recall the coefficients $r_{i}$ (for $i \in I_{0}$ ) in the expression $\theta=\sum_{i \in I_{0}} r_{i} \alpha_{i}$ (note that we correct here a

\begin{tabular}{|c|c|c|c|}
\hline$X_{\tilde{n}=\tilde{n}(n)}^{(k)}$ & $n$ & $(\Gamma, I)$ & $\left(r_{1}, \ldots, r_{n}\right)$ \\
\hline$A_{1}^{(1)}$ & 1 & $\stackrel{\circ}{=}{ }_{1}^{\circ}$ & (1) \\
\hline$A_{n}^{(1)}$ & $>1$ & ${ }_{1}^{\circ}-O_{2}^{0} \cdot n_{n-1}^{\circ}$ & $(1, \ldots, 1)$ \\
\hline$B_{n}^{(1)}$ & $>2$ & ${ }_{1}^{\circ}<=_{2}^{\circ}-o_{3}^{\circ} \cdots \stackrel{\circ-2 n-1}{\circ}{ }_{n}^{\circ}$ & $(2, \ldots, 2,1)$ \\
\hline$C_{n}^{(1)}$ & $>1$ & $\stackrel{\circ}{=}>_{2}-{ }_{3}^{\circ}$. & $(1,2, \ldots, 2)$ \\
\hline
\end{tabular}
misprint in [Da]: the coefficient $r_{n}$ for case $A_{2 n-1}^{(2)}$ ). 


\begin{tabular}{|c|c|c|c|}
\hline$D_{n}^{(1)}$ & $>3$ & 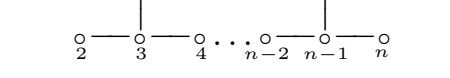 & $(1,1,2, \ldots, 2,1)$ \\
\hline$E_{6}^{(1)}$ & 6 & $\stackrel{\circ}{\circ}-{ }_{3}^{\circ}-\bigcirc_{4}^{1}-{ }_{5}^{\circ}-\circ$ & $(2,1,2,3,2,1)$ \\
\hline$E_{7}^{(1)}$ & 7 & $\stackrel{\circ}{\circ}-{ }_{2}^{\circ}-{ }_{3}^{\circ}-{ }_{4}^{\circ}-{ }_{5}^{\circ}-\underset{6}{\circ}-{ }_{7}^{\circ}$ & $(2,2,3,4,3,2,1)$ \\
\hline$E_{8}^{(1)}$ & 8 & ${ }_{2}^{\circ}-\mathrm{o}_{3}-\mathrm{o}_{4}^{\circ}-{ }_{5}^{\circ}-{ }_{6}^{\circ}-{ }_{7}^{\circ}-{ }_{8}^{\circ}$ & $(3,2,4,6,5,4,3,2)$ \\
\hline$F_{4}^{(1)}$ & 4 & $\stackrel{\circ}{\circ}-\stackrel{\circ}{\circ}<=\stackrel{\circ}{\circ}-\stackrel{\circ}{\circ}-\stackrel{\circ}{\circ}$ & $(2,4,3,2)$ \\
\hline$G_{2}^{(1)}$ & 2 & $\stackrel{\circ}{1}<\equiv \equiv_{2}^{\circ}-\underset{0}{\circ}$ & $(3,2)$ \\
\hline$A_{2}^{(2)}$ & 1 & $\circ<\equiv{ }_{0}$ & $(2)$ \\
\hline$A_{2 n}^{(2)}$ & $>1$ & ${ }_{1}^{\circ}<=\underset{2}{\circ}-{ }_{3}^{\circ} \ldots \underset{n-1}{\circ}{ }_{n}^{\circ}<=$ & $(2, \ldots, 2)$ \\
\hline$A_{2 n-1}^{(2)}$ & $>2$ & $\stackrel{\circ}{=}>>\circ-{ }_{2}^{\circ} \ldots \stackrel{\circ-2 n-1}{\circ}-\stackrel{\circ}{\circ}$ & $(1,2, \ldots, 2,1)$ \\
\hline$D_{n+1}^{(2)}$ & $>1$ & ${ }_{1}^{\circ}<={ }_{2}^{\circ}-o_{3}^{\circ} \cdots{ }_{n-1}^{\circ}{ }_{n}^{\circ}=>>_{0}^{\circ}$ & $(1, \ldots, 1)$ \\
\hline$E_{6}^{(2)}$ & 4 & $\stackrel{\circ}{\circ}-\stackrel{\circ}{\circ}-\underset{2}{\circ}<=\stackrel{\circ}{\circ}-{ }_{4}^{\circ}$ & $(2,3,2,1)$ \\
\hline$D_{4}^{(3)}$ & 2 & $\circ-\circ<\equiv{ }_{0}^{\circ}$ & $(2,1)$ \\
\hline
\end{tabular}

\section{§2. Preliminaries: Weyl group and root system}

The following structures of the affine Weyl group and root system (see [Bo], [IM], $[\mathrm{K}],[\mathrm{M}])$ will be used in the paper:

(i) the Weyl group $W_{0}=\left\langle s_{i} \mid i \in I_{0}\right\rangle \subseteq \operatorname{Aut}\left(Q_{0}\right)$ of $\Gamma_{0}$ acts on $Q$ by $s_{i}\left(\alpha_{j}\right)=$ $\alpha_{j}-a_{i j} \alpha_{i}$ for $i \in I_{0}, j \in I$ and this action extends to the Weyl group $W=\left\langle s_{i} \mid i \in I\right\rangle \subseteq \operatorname{Aut}(Q)$ of $\Gamma$ by $s_{0}\left(\alpha_{i}\right)=\alpha_{i}-a_{0 i} \alpha_{0}$ for $i \in I ;$

(ii) the $W$-invariant bilinear form $(\cdot \mid \cdot)$ on $Q$ induces a positive semidefinite symmetric bilinear form on $\mathbb{R} \otimes_{\mathbb{Z}} Q$ : it is obviously positive definite on $\mathbb{R} \otimes_{\mathbb{Z}} Q_{0}$, 
and has kernel generated by $\delta=\alpha_{0}+\theta=\sum_{i \in I} r_{i} \alpha_{i} \in Q$ where $r_{0}=1$ always;

(iii) $(\cdot \mid \cdot)$ can be uniquely normalized in such a way that there is a diagonal matrix $D=\operatorname{diag}\left(d_{i} \mid i \in I\right)$ with $1 \in\left\{d_{i} \mid i \in I_{0}\right\} \subseteq\left\{d_{i} \mid i \in I\right\} \subseteq \mathbb{Z}_{+}$and $\left(\alpha_{i} \mid \alpha_{j}\right)=d_{i} a_{i j}$ for all $i, j \in I$; for $i \in I, w \in W$ set $d_{w\left(\alpha_{i}\right)}=d_{i}$;

(iv) for $i \in I_{0}$ define $\tilde{d}_{i}= \begin{cases}1 & \text { if } k=1 \text { or } X_{\tilde{n}}^{(k)}=A_{2 n}^{(2)}, \\ d_{i} & \text { otherwise; }\end{cases}$

(v) the weight lattice $\hat{P} \subseteq \mathbb{R} \otimes_{Z} Q_{0}$ is $\hat{P}=\bigoplus_{i \in I_{0}} \mathbb{Z} \lambda_{i}$, where for all $i \in I_{0}$, $\lambda_{i} \in \mathbb{R} \otimes_{\mathbb{Z}} Q_{0}$ is defined by $\left(\lambda_{i} \mid \alpha_{j}\right)=\tilde{d}_{i} \delta_{i j}$ for all $j \in I_{0} ; Q_{0}$ naturally embeds in $\hat{P}$, which provides a $W$-invariant action on $Q$ by $x(\alpha)=\alpha-(x \mid \alpha) \delta$ for $x \in \hat{P}$ and $\alpha \in Q$;

(vi) as subgroups of $\operatorname{Aut}(Q)$ we have $W \leq \hat{P} \rtimes W_{0} ; \hat{W}=\hat{P} \rtimes W_{0}$ is called the extended Weyl group of $\Gamma$ and we also have $\hat{W}=W \rtimes \mathcal{T}$, where $\mathcal{T}=$ $\operatorname{Aut}(\Gamma) \cap \hat{W}$

(vii) the extended braid group $\hat{\mathcal{B}}$ is the group generated by $\left\{T_{w} \mid w \in \hat{W}\right\}$ with relations $T_{w} T_{w^{\prime}}=T_{w w^{\prime}}$ whenever $l\left(w w^{\prime}\right)=l(w) l\left(w^{\prime}\right)$, where $l: \hat{W} \rightarrow \mathbb{N}$ is defined by

$l(w)=\min \left\{r \in \mathbb{N} \mid \exists i_{1}, \ldots, i_{r} \in I\right.$ and $\tau \in \mathcal{T}$ such that $\left.w=s_{i_{1}} \cdot \ldots \cdot s_{i_{r}} \tau\right\}$

set $T_{i}=T_{s_{i}}$ for $i \in I$; recall that $l\left(\sum_{i \in I_{0}} m_{i} \lambda_{i}\right)=\sum_{i \in I_{0}} m_{i} l\left(\lambda_{i}\right)$ if $m_{i} \in \mathbb{N}$ for all $i \in I_{0}$;

(viii) the root system $\Phi$ of $\Gamma$ decomposes into the union of the sets $\Phi^{\text {re }}$ of real roots and $\Phi^{\mathrm{im}}$ of imaginary roots, where $\Phi^{\mathrm{re}}$ is the $W$-orbit in $Q$ of the set $\left\{\alpha_{i} \mid i \in I\right\}$ and $\Phi^{\mathrm{im}}=\{m \delta \mid m \in \mathbb{Z} \backslash\{0\}\}$; the set of positive roots is $\Phi_{+}=\Phi \cap Q_{+} ;$

(ix) the multiplicity of the root $\alpha \in \Phi$ is 1 if $\alpha$ is real and $\#\left\{i \in I_{0}\left|\tilde{d}_{i}\right| m\right\}$ if $\alpha=m \delta(m \in \mathbb{Z} \backslash\{0\})$; the set $\hat{\Phi}$ of roots with multiplicities is $\hat{\Phi}=\Phi^{\mathrm{re}} \cup \hat{\Phi}^{\mathrm{im}}$ where $\left.\hat{\Phi}^{\mathrm{im}}=\left\{(m \delta, i) \mid i \in I_{0}, m \in \mathbb{Z} \backslash\{0\}\right\}, \tilde{d}_{i} \mid m\right\}$; the set of positive roots with multiplicities is $\hat{\Phi}_{+}=\Phi_{+}^{\mathrm{re}} \cup \hat{\Phi}_{+}^{\mathrm{im}}=\left(\Phi_{+} \cap \Phi^{\mathrm{re}}\right) \cup\{(m \delta, i) \in \hat{\Phi} \mid m>0\}$;

(x) choose a sequence $\iota: \mathbb{Z} \ni r \mapsto \iota_{r} \in I$ such that $s_{\iota_{1}} \cdot \ldots \cdot s_{\iota_{N_{i}}} \tau_{i}=\sum_{j=1}^{i} \lambda_{j}$ for all $i \in I_{0}$ and $\iota_{r+N_{n}}=\tau_{n}\left(\iota_{r}\right)$ for all $r \in \mathbb{Z}$, where $N_{i}=\sum_{j=1}^{i} l\left(\lambda_{j}\right)$ and $\tau_{i} \in \mathcal{T}$; then $\iota$ induces a map

$$
\mathbb{Z} \ni r \mapsto w_{r} \in W \quad \text { defined by } \quad w_{r}= \begin{cases}s_{\iota_{1}} \cdot \ldots \cdot s_{\iota_{r-1}} & \text { if } r \geq 1, \\ s_{\iota_{0}} \cdot \ldots \cdot s_{\iota_{r+1}} & \text { if } r \leq 0,\end{cases}
$$

and a bijection

$$
\mathbb{Z} \ni r \mapsto \beta_{r}=w_{r}\left(\alpha_{\iota_{r}}\right) \in \Phi_{+}^{\mathrm{re}} ;
$$


(xi) the total ordering $\preceq$ of $\hat{\Phi}_{+}$defined by

$$
\begin{gathered}
\beta_{r} \preceq \beta_{r-1} \preceq(\tilde{m} \delta, i) \preceq(m \delta, j) \preceq(m \delta, i) \preceq \beta_{s+1} \preceq \beta_{s} \\
\forall r \leq 0, s \geq 1, \tilde{m}>m>0, j \leq i \in I_{0}
\end{gathered}
$$

induces on $\Phi_{+}$a convex ordering: if $\alpha=\sum_{r=1}^{M} \gamma_{r}$ with $M>1, \gamma_{1} \preceq \cdots \preceq \gamma_{M}$ and $\alpha, \gamma_{r} \in \Phi_{+}$for all $r=1, \ldots, M$, then either $\gamma_{1} \prec \alpha$ or $\gamma_{r} \in \Phi^{\text {im }}$ for all $r=1, \ldots, M$.

\section{§3. Preliminaries: the Drinfeld-Jimbo presentation $\mathcal{U}_{q}$}

In this section we recall the definition of the quantum algebra $\mathcal{U}_{q}$ introduced by Drinfeld and Jimbo (see [Dr2] and [Jm]), and the structures and results (see [Be], $[\mathrm{Da}],[\mathrm{LS}],[\mathrm{L}])$ needed in $\S 12$. First of all recall some notation.

Notation 3.1. (i) For all $i \in I_{0}$ we denote by $q_{i}$ the element $q_{i}=q^{d_{i}} \in \mathbb{C}(q)$.

(ii) Consider the ring $\mathbb{Z}\left[x, x^{-1}\right]$. Then for all $m, r \in \mathbb{Z}$ the elements $[m]_{x},[m]_{x}$ ! $(m \geq 0)$ and $\left[\begin{array}{c}m \\ r\end{array}\right]_{x}(m \geq r \geq 0)$ of $\mathbb{Z}\left[x, x^{-1}\right]$ are defined by $[m]_{x}=\frac{x^{m}-x^{-m}}{x-x^{-1}}$, $[m]_{x} !=\prod_{s=1}^{m}[s]_{x}$ and $\left[\begin{array}{c}m \\ r\end{array}\right]_{x}=\frac{[m]_{x} !}{[r]_{x} ![m-r]_{x} !}$.

(iii) Consider the field $\mathbb{C}(q)$ and, given $v \in \mathbb{C}(q) \backslash\{0\}$, the natural homomorphism $\mathbb{Z}\left[x, x^{-1}\right] \rightarrow \mathbb{C}(q)$ determined by the condition $x \mapsto v$; then for all $m, r \in \mathbb{Z}$ $[m]_{v},[m]_{v} !(m \geq 0)$ and $\left[\begin{array}{c}m \\ r\end{array}\right]_{v}(m \geq r \geq 0)$ denote the images in $\mathbb{C}(q)$ of $[m]_{x}$, $[m]_{x}$ ! and $\left[\begin{array}{c}m \\ r\end{array}\right]_{x}$ respectively.

Definition 3.2. Let $\Gamma=(I, A)$ be a Dynkin diagram of finite or affine type.

(i) The (Drinfeld-Jimbo) quantum algebra of type $\Gamma$ is the $\mathbb{C}(q)$-algebra $\mathcal{U}_{q}=$ $\mathcal{U}_{q}(\Gamma)$ generated by

$$
\left\{E_{i}, F_{i}, K_{i}^{ \pm 1} \mid i \in I\right\}
$$

with relations

$$
\begin{aligned}
& K_{i} K_{i}^{-1}=1=K_{i}^{-1} K_{i}, \quad K_{i} K_{j}=K_{j} K_{i} \quad \forall i, j \in I, \\
& K_{i} E_{j}=q_{i}^{a_{i j}} E_{j} K_{i}, \quad K_{i} F_{j}=q_{i}^{-a_{i j}} F_{j} K_{i} \quad \forall i, j \in I, \\
& {\left[E_{i}, F_{j}\right]=\delta_{i j} \frac{K_{i}-K_{i}^{-1}}{q_{i}-q_{i}^{-1}} \quad \forall i, j \in I,} \\
& \sum_{u=0}^{1-a_{i j}}\left[\begin{array}{c}
1-a_{i j} \\
u
\end{array}\right]_{q_{i}} E_{i}^{u} E_{j} E_{i}^{1-a_{i j}-u}=0 \quad \forall i \neq j \in I, \\
& \sum_{u=0}^{1-a_{i j}}\left[\begin{array}{c}
1-a_{i j} \\
u
\end{array}\right]_{q_{i}} F_{i}^{u} F_{j} F_{i}^{1-a_{i j}-u} \quad \forall i \neq j \in I ;
\end{aligned}
$$

the last two sets of relations are called the Serre relations. 
If $\Gamma$ is affine of type $X_{\tilde{n}}^{(k)}$ we also set:

(ii) $\mathcal{U}_{q}^{\mathrm{DJ}}=\mathcal{U}_{q}^{\mathrm{DJ}}\left(X_{\tilde{n}}^{(k)}\right)=\mathcal{U}_{q}(\Gamma)$, to stress the distinction of this affine quantum algebra from its Drinfeld realization;

(iii) $\mathcal{U}_{q}^{\mathrm{fin}}=\mathcal{U}_{q}^{\mathrm{fin}}\left(X_{\tilde{n}}^{(k)}\right)=\mathcal{U}_{q}\left(\Gamma_{0}\right)(\operatorname{see} \S 1(\mathrm{I}))$

Definition 3.3. Recall that $\mathcal{U}_{q}$ is endowed with the following structures:

(i) the $Q$-gradation $\mathcal{U}_{q}=\bigoplus_{\alpha \in Q} \mathcal{U}_{q, \alpha}$ determined by the conditions:

$$
\begin{aligned}
& E_{i} \in \mathcal{U}_{q, \alpha_{i}}, \quad F_{i} \in \mathcal{U}_{q,-\alpha_{i}}, \quad K_{i}^{ \pm 1} \in \mathcal{U}_{q, 0} \quad \forall i \in I \\
& \mathcal{U}_{q, \alpha} \mathcal{U}_{q, \beta} \subseteq \mathcal{U}_{q, \alpha+\beta} \quad \forall \alpha, \beta \in Q ;
\end{aligned}
$$

(ii) the triangular decomposition: $\mathcal{U}_{q} \cong \mathcal{U}_{q}^{-} \otimes \mathcal{U}_{q}^{0} \otimes \mathcal{U}_{q}^{+}$, where $\mathcal{U}_{q}^{-}, \mathcal{U}_{q}^{0}$ and $\mathcal{U}_{q}^{+}$are the subalgebras of $\mathcal{U}_{q}$ generated respectively by $\left\{E_{i} \mid i \in I\right\},\left\{K_{i}^{ \pm 1} \mid i \in I\right\}$ and $\left\{F_{i} \mid i \in I\right\}$; in particular

$$
\mathcal{U}_{q, \alpha} \cong \bigoplus_{\beta, \gamma \in Q_{+}: \gamma-\beta=\alpha} \mathcal{U}_{q,-\beta}^{-} \otimes \mathcal{U}_{q}^{0} \otimes \mathcal{U}_{q, \gamma}^{+} \quad \forall \alpha \in Q
$$

where $\mathcal{U}_{q, \alpha}^{ \pm}=\mathcal{U}_{q, \alpha} \cap \mathcal{U}_{q}^{ \pm}$

(iii) the $\mathbb{C}$-antilinear antiinvolution $\Omega: \mathcal{U}_{q} \rightarrow \mathcal{U}_{q}$ defined by

$$
\Omega(q)=q^{-1} ; \quad \Omega\left(E_{i}\right)=F_{i}, \quad \Omega\left(F_{i}\right)=E_{i}, \quad \Omega\left(K_{i}\right)=K_{i}^{-1} \quad \forall i \in I ;
$$

(iv) the $\mathbb{C}(q)$-linear antiinvolution $\Xi: \mathcal{U}_{q} \rightarrow \mathcal{U}_{q}$ defined by

$$
\Xi\left(E_{i}\right)=E_{i}, \quad \Xi\left(F_{i}\right)=F_{i}, \quad \Xi\left(K_{i}\right)=K_{i}^{-1} \quad \forall i \in I_{;}
$$

(v) the braid group action defined by

$$
\begin{gathered}
T_{i}\left(K_{j}\right)=K_{j} K_{i}^{-a_{i j}} \quad \forall i, j \in I, \\
T_{i}\left(E_{i}\right)=-F_{i} K_{i}, \quad T_{i}\left(F_{i}\right)=-K_{i}^{-1} E_{i} \quad \forall i \in I, \\
T_{i}\left(E_{j}\right)=\sum_{r=0}^{-a_{i j}}(-1)^{r-a_{i j}} q_{i}^{-r} E_{i}^{\left(-a_{i j}-r\right)} E_{j} E_{i}^{(r)}, T_{i}\left(F_{j}\right)=\Omega\left(T_{i}\left(E_{j}\right)\right) \forall i \neq j \in I
\end{gathered}
$$

where $E_{i}^{(m)}=E_{i}^{m} /[m]_{q_{i}}$ ! for $m \in \mathbb{N}$;

(vi) a natural Aut( $\Gamma)$-action: $\tau\left(K_{i}\right)=K_{\tau(i)}, \tau\left(E_{i}\right)=E_{\tau(i)}, \tau\left(F_{i}\right)=F_{\tau(i)}$ for all $\tau \in \operatorname{Aut}(\Gamma)$ and $i \in I_{0}$; if $\Gamma$ is affine then setting $T_{\tau}=\tau$ extends the braid group action to an extended braid group action;

(vii) if $\Gamma \hookrightarrow \Gamma^{\prime}$ is a Dynkin diagram embedding then the $\mathbb{C}$-homomorphism

$$
\varphi_{\Gamma, \Gamma^{\prime}}: \mathcal{U}_{q}(\Gamma) \rightarrow \mathcal{U}_{q}\left(\Gamma^{\prime}\right)
$$


is naturally defined by

$$
q \mapsto q^{\min \left\{d_{i}^{\prime} \mid i \in I\right\}}, \quad K_{i}^{ \pm 1} \mapsto K_{i}^{ \pm 1}, \quad E_{i} \mapsto E_{i}, \quad F_{i} \mapsto F_{i} \quad(i \in I) ;
$$

in particular if $\Gamma$ is of affine type, $\varphi=\varphi_{\Gamma_{0}, \Gamma}: \mathcal{U}_{q}^{\text {fin }} \rightarrow \mathcal{U}_{q}^{\text {DJ }}$ is a $\mathbb{C}(q)$ homomorphism;

(viii) positive and negative root vectors $E_{\alpha} \in \mathcal{U}_{q, \alpha}^{\mathrm{DJ},+}$ and $F_{\alpha}=\Omega\left(E_{\alpha}\right) \in \mathcal{U}_{q,-\alpha}^{\mathrm{DJ},-}$ ( $\left.\alpha \in \hat{\Phi}_{+}\right)$such that if $\Gamma$ is of affine type then $E_{\beta_{r}}=T_{w_{r}}\left(E_{\iota_{r}}\right)$ for $r \geq 1$, $E_{\beta_{r}}=T_{w_{r}^{-1}}^{-1}\left(E_{\iota_{r}}\right)$ for $r \leq 0$, and $E_{\left(\tilde{d}_{i} r \delta, i\right)}=-E_{\tilde{d}_{i} r \delta-\alpha_{i}} E_{i}+q_{i}^{-2} E_{i} E_{\tilde{d}_{i} r \delta-\alpha_{i}}$ for $r>0$ and $i \in I_{0}$.

Remark 3.4. (i) $\Omega \Xi=\Xi \Omega, \Omega T_{i}=T_{i} \Omega$ for all $i \in I$ and $\Omega \tau=\tau \Omega$ for all $\tau \in \mathcal{T}$;

(ii) $\Xi T_{i}=T_{i}^{-1} \Xi$ for all $i \in I$ and $\Xi \tau=\tau \Xi$ for all $\tau \in \mathcal{T}$;

moreover if $\Gamma$ is of affine type:

(iii) $\varphi$ commutes with $\Omega, \Xi$ and $T_{i}\left(i \in I_{0}\right)$;

(iv) in cases $A_{1}^{(1)}$ and $A_{2}^{(2)}, \Xi T_{1} T_{\lambda_{1}}=T_{\lambda_{1}}^{-1} \Xi T_{1}$ (recall that $T_{\lambda_{1}}=T_{0} T_{\tau}=T_{\tau} T_{1}$, where $\langle\tau\rangle=\operatorname{Aut}(\Gamma)$ in case $A_{1}^{(1)}$, and $T_{\lambda_{1}}=T_{0} T_{1}$ in case $\left.A_{2}^{(2)}\right)$;

(v) $T_{w}\left(\mathcal{U}_{q, \alpha}^{\mathrm{DJ}}\right)=\mathcal{U}_{q, w(\alpha)}^{\mathrm{DJ}}$ for all $w \in \hat{W}, \alpha \in Q$;

(vi) $T_{w}\left(E_{i}\right) \in \mathcal{U}_{q, w\left(\alpha_{i}\right)}^{\mathrm{DJ},+}$ if $w \in \hat{W}$ and $i \in I$ are such that $w\left(\alpha_{i}\right) \in Q_{+}$(i.e. $\left.l\left(w s_{i}\right)>l(w)\right)$

(vii) $E_{m \tilde{d}_{i} \delta+\alpha_{i}}=T_{\lambda_{i}}^{-m}\left(E_{i}\right)$ and $F_{m \tilde{d}_{i} \delta+\alpha_{i}}=T_{\lambda_{i}}^{-m}\left(F_{i}\right)$ for all $m \in \mathbb{N}$ and $i \in I_{0}$;

(viii) $\left\{K_{\alpha} \mid \alpha \in Q\right\}$ is a basis of $\mathcal{U}_{q}^{\mathrm{DJ}, 0}$, where $K_{\alpha}=\prod_{i \in I} K_{i}^{m_{i}}$ if $\alpha=\sum_{i \in I} m_{i} \alpha_{i}$ $\in Q$

(ix) $\left\{E(\gamma)=E_{\gamma_{1}} \cdot \ldots \cdot E_{\gamma_{M}} \mid M \in \mathbb{N}, \gamma=\left(\gamma_{1} \preceq \cdots \preceq \gamma_{M}\right), \gamma_{h} \in \hat{\Phi}_{+}\right.$ $\forall h=1, \ldots, M\}$ is a basis of $\mathcal{U}_{q}^{\mathrm{DJ},+}$;

(x) $\left\{E(\gamma) K_{\alpha} \Omega\left(E\left(\gamma^{\prime}\right)\right) \mid \alpha \in Q, \gamma=\left(\gamma_{1} \preceq \cdots \preceq \gamma_{M}\right) \in \hat{\Phi}_{+}^{M}, \gamma^{\prime}=\left(\gamma_{1}^{\prime} \preceq \cdots \preceq \gamma_{M^{\prime}}^{\prime}\right)\right.$ $\left.\in \hat{\Phi}_{+}^{M^{\prime}}, M, M^{\prime} \in \mathbb{N}\right\}$ is a basis of $\mathcal{U}_{q}^{\mathrm{DJ}}$, called the $P B W$-basis;

(xi) for all $\alpha \prec \beta \in \hat{\Phi}_{+} E_{\beta} E_{\alpha}-q^{(\alpha \mid \beta)} E_{\alpha} E_{\beta}$ is a linear combination of $\{E(\gamma) \mid \gamma=$ $\left.\left(\gamma_{1} \preceq \cdots \preceq \gamma_{M}\right) \in \hat{\Phi}_{+}^{M}, M \in \mathbb{N}, \alpha \prec \gamma_{1}\right\}$ (Levendorskii-Soibelman formula).

Remark 3.5. If $\Gamma$ is affine Remark 3.4(ix) implies that $\operatorname{dim} \mathcal{U}_{q, \alpha}^{\mathrm{DJ},+}=\operatorname{dim} \mathcal{U}_{q, \alpha}^{\mathrm{fin},+}$ for all $\alpha \in Q_{0,+}$. In particular $\varphi$ is injective.

Definition 3.6. If $\Gamma$ is affine, for $i \in I_{0}$ let

$$
\varphi_{i}: \begin{cases}\mathcal{U}_{q}^{\mathrm{DJ}}\left(A_{1}^{(1)}\right) \rightarrow \mathcal{U}_{q}^{\mathrm{DJ}}\left(X_{\tilde{n}}^{(k)}\right) & \text { if }\left(X_{\tilde{n}}^{(k)}, i\right) \neq\left(A_{2 n}^{(2)}, 1\right), \\ \mathcal{U}_{q}^{\mathrm{DJ}}\left(A_{2}^{(2)}\right) \rightarrow \mathcal{U}_{q}^{\mathrm{DJ}}\left(X_{\tilde{n}}^{(k)}\right) & \text { if }\left(X_{\tilde{n}}^{(k)}, i\right)=\left(A_{2 n}^{(2)}, 1\right),\end{cases}
$$

be the $\mathbb{C}$-homomorphisms defined on the generators as follows:

$$
q \mapsto q_{i}, \quad K_{1}^{ \pm 1} \mapsto K_{i}^{ \pm 1}, \quad E_{1} \mapsto E_{i}, \quad F_{1} \mapsto F_{i}
$$


and

$$
\begin{array}{llll}
K_{0} \mapsto K_{\tilde{d}_{i} \delta-\alpha_{i}}, & E_{0} \mapsto E_{\tilde{d}_{i} \delta-\alpha_{i}}, & F_{0} \mapsto F_{\tilde{d}_{i} \delta-\alpha_{i}} & \text { if }\left(X_{\tilde{n}}^{(k)}, i\right) \neq\left(A_{2 n}^{(2)}, 1\right), \\
K_{0} \mapsto K_{\delta-2 \alpha_{1}}, & E_{0} \mapsto E_{\delta-2 \alpha_{1}}, & F_{0} \mapsto F_{\delta-2 \alpha_{1}} & \text { if }\left(X_{\tilde{n}}^{(k)}, i\right)=\left(A_{2 n}^{(2)}, 1\right) .
\end{array}
$$

Remark 3.7. (i) $\varphi_{i} \Omega=\Omega \varphi_{i}, \varphi_{i} T_{1}=T_{i} \varphi_{i}$ and $\varphi_{i} T_{\lambda_{1}}=T_{\lambda_{i}} \varphi_{i}$ for all $i \in I_{0}$;

(ii) $\varphi_{i}\left(i \in I_{0}\right)$ is injective (thanks to the PBW-bases).

\section{$\S 4$. The Drinfeld realization $\mathcal{U}_{q}^{\mathrm{Dr}}$ : definition}

In this section the definition of the Drinfeld realization $\mathcal{U}_{q}^{\mathrm{Dr}}\left(X_{\tilde{n}}^{(k)}\right)$ of the affine quantum algebra of type $X_{\tilde{n}}^{(k)}$ is presented; the definition is discussed and reformulated using the set $I_{0} \times \mathbb{Z}$ as index set for the generators instead of the set $\tilde{I} \times \mathbb{Z}$ used in [Dr1] and followed in the literature (see for instance [CP3], [Jn], [JZ2]), because the relations translated from $\tilde{I} \times \mathbb{Z}$ to $I_{0} \times \mathbb{Z}$ seem simpler to handle, even though they lose the immediate connection with the datum $(\tilde{I}, \chi)$. This reformulation, which is useful if one aims to compare the Drinfeld realization with the Drinfeld--Jimbo presentation, is not difficult, but it is presented with some care in order to avoid any ambiguity.

Notation 4.1. (i) $\omega$ denotes a primitive $k^{\text {th }}$ root of 1 .

(ii) Fix the normalization of the $\tilde{W}$-invariant bilinear form $(\cdot \mid \cdot)$ on $\tilde{Q}$ such that $\min \left\{\sum_{u \in \mathbb{Z} / k \mathbb{Z}}\left(\tilde{\alpha}_{i^{\prime}} \mid \tilde{\alpha}_{\chi^{u}\left(i^{\prime}\right)}\right) \mid i^{\prime} \in \tilde{I}\right\}=2$.

(iii) Denote $\tilde{d}=\max \left\{\tilde{d}_{i} \mid i \in I_{0}\right\}$ (in case $A_{2 n}^{(2)}, \tilde{d}=1$, otherwise $\tilde{d}=k$ ).

(iv) Let $Y$ be a function from $\mathbb{Z}^{l}(l \in \mathbb{N})$ to any algebra; given $\sigma \in \mathcal{S}_{l}$ and $p=\left(p_{1}, \ldots, p_{l}\right) \in \mathbb{Z}^{l}$ set $\sigma \cdot(Y(p))=Y(\sigma \cdot p)=Y\left(p_{\sigma^{-1}(1)}, \ldots, p_{\sigma^{-1}(l)}\right)$.

(v) Analogously if $f \in \mathbb{C}(q)\left[\left[u_{1}^{ \pm 1}, \ldots, u_{l}^{ \pm 1}\right]\right]$ and $u=\left(u_{1}, \ldots, u_{l}\right)$ define $\sigma \cdot(f(u))$ by $\sigma \cdot(f(u))=f\left(u_{\sigma^{-1}(1)}, \ldots, u_{\sigma^{-1}(l)}\right)$ for all $\sigma \in \mathcal{S}_{l}$.

(vi) By " $\left(R^{ \pm}\right)$is the relation $S^{ \pm}=0$ " is meant that " $\left(R^{+}\right)$is the relation $S^{+}=0$ and $\left(R^{-}\right)$is the relation $S^{-}=0$ ".

(vii) More generally " $A^{ \pm}$has property $P^{ \pm}$" means " $A^{+}$has property $P^{+}$and $A^{-}$ has property $P^{-"}$.

For the definition of the Drinfeld realization of affine quantum algebras, which we recall here, see [Dr1].

Definition 4.2. Let $X_{\tilde{n}}^{(k)}$ be a Dynkin diagram of affine type; the Drinfeld realization of the quantum algebra of type $X_{\tilde{n}}^{(k)}$ is the $\mathbb{C}(q)$-algebra $\mathcal{U}_{q}^{\operatorname{Dr}}\left(X_{\tilde{n}}^{(k)}\right)=\mathcal{U}_{q}^{\operatorname{Dr}}$ generated by

(G) $\quad \mathcal{C}^{ \pm 1}, \quad \mathcal{K}_{i^{\prime}}^{ \pm 1}\left(i^{\prime} \in \tilde{I}\right), \quad \mathcal{X}_{i^{\prime}, r}^{ \pm}\left(\left(i^{\prime}, r\right) \in \tilde{I} \times \mathbb{Z}\right), \quad \mathcal{H}_{i^{\prime}, r}\left(\left(i^{\prime}, r\right) \in \tilde{I} \times(\mathbb{Z} \backslash\{0\})\right)$, 
with the following relations $(\mathcal{D} \mathcal{R})$ :

$$
\begin{gathered}
\mathcal{K}_{\chi\left(i^{\prime}\right)}=\mathcal{K}_{i^{\prime}}, \quad \mathcal{H}_{\chi\left(i^{\prime}\right), r}=\omega^{r} \mathcal{H}_{i^{\prime}, r} \quad\left(i^{\prime} \in \tilde{I}, r \in \mathbb{Z} \backslash\{0\}\right), \\
\mathcal{X}_{\chi\left(i^{\prime}\right), r}^{ \pm}=\omega^{r} \mathcal{X}_{i^{\prime}, r}^{ \pm} \quad\left(\left(i^{\prime}, r\right) \in \tilde{I} \times \mathbb{Z}\right), \\
\mathcal{C C}^{-1}=1, \quad[\mathcal{C}, x]=0 \quad \forall x,
\end{gathered}
$$

$\left(\mathcal{K} \mathcal{X}^{ \pm}\right) \quad \mathcal{K}_{i^{\prime}} \mathcal{X}_{j^{\prime}, r}^{ \pm}=q^{ \pm \sum_{u \in \mathbb{Z} / k \mathbb{Z}}\left(\tilde{\alpha}_{i^{\prime}} \mid \tilde{\alpha}_{\chi^{u}\left(j^{\prime}\right)}\right)} \mathcal{X}_{j^{\prime}, r}^{ \pm} \mathcal{K}_{i^{\prime}} \quad\left(i^{\prime}, j^{\prime} \in \tilde{I}, r \in \mathbb{Z}\right)$,

$$
\left[\mathcal{K}_{i^{\prime}}, \mathcal{H}_{j^{\prime}, r}\right]=0 \quad\left(i^{\prime}, j^{\prime} \in \tilde{I}, r \in \mathbb{Z} \backslash\{0\}\right),
$$

$$
\begin{array}{r}
{\left[\mathcal{X}_{i^{\prime}, r}^{+}, \mathcal{X}_{j^{\prime}, s}^{-}\right]=\frac{\sum_{u=0}^{k-1} \delta_{\chi^{u}\left(i^{\prime}\right), j^{\prime}} \omega^{u s}}{\sum_{u=0}^{k-1} \delta_{\chi^{u}\left(i^{\prime}\right), i^{\prime}}} \cdot \frac{\mathcal{C}^{-s} \mathcal{K}_{i^{\prime}} \tilde{\mathcal{H}}_{i^{\prime}, r+s}^{+}-\mathcal{C}^{-r} \mathcal{K}_{i^{\prime}}^{-1} \tilde{\mathcal{H}}_{i^{\prime}, r+s}^{-}}{\left(q-q^{-1}\right)\left[\frac{1}{2} \sum_{u \in \mathbb{Z} / k \mathbb{Z}}\left(\tilde{\alpha}_{\chi^{u}\left(i^{\prime}\right)} \mid \tilde{\alpha}_{i^{\prime}}\right)\right]_{q}}} \\
\left(\left(i^{\prime}, r\right),\left(j^{\prime}, s\right) \in \tilde{I} \times \mathbb{Z}\right),
\end{array}
$$

$\left(\mathcal{H} \mathcal{X}^{ \pm}\right)$

$$
\left[\mathcal{H}_{i^{\prime}, r}, \mathcal{X}_{j^{\prime}, s}^{ \pm}\right]= \pm \tilde{b}_{i^{\prime} j^{\prime} r} \mathcal{C}^{\frac{r \mp|r|}{2}} \mathcal{X}_{j^{\prime}, r+s}^{ \pm} \quad\left(\left(i^{\prime}, r\right) \in \tilde{I} \times(\mathbb{Z} \backslash\{0\}),\left(j^{\prime}, s\right) \in \tilde{I} \times \mathbb{Z}\right),
$$

$$
\begin{array}{r}
{\left[\mathcal{H}_{i^{\prime}, r}, \mathcal{H}_{j^{\prime}, s}\right]=\delta_{r+s, 0} \tilde{b}_{i^{\prime} j^{\prime} r} \frac{C^{r}-C^{-r}}{\left.\left(q-q^{-1}\right)\left[\frac{1}{2} \sum_{u \in \mathbb{Z} / k \mathbb{Z}}\left(\tilde{\alpha}_{\chi^{u}\left(j^{\prime}\right)}\right) \tilde{\alpha}_{j^{\prime}}\right)\right]_{q}}} \\
\left(\left(i^{\prime}, r\right),\left(j^{\prime}, s\right) \in \tilde{I} \times(\mathbb{Z} \backslash\{0\})\right),
\end{array}
$$

$\left(\mathcal{X} \mathcal{F} \mathcal{G}^{ \pm}\right) \quad F_{i^{\prime} j^{\prime}}^{ \pm}\left(u_{1}, u_{2}\right) \mathcal{X}_{i^{\prime}}^{ \pm}\left(u_{1}\right) \mathcal{X}_{j^{\prime}}^{ \pm}\left(u_{2}\right)=G_{i^{\prime} j^{\prime}}^{ \pm}\left(u_{1}, u_{2}\right) \mathcal{X}_{j^{\prime}}^{ \pm}\left(u_{2}\right) \mathcal{X}_{i^{\prime}}^{ \pm}\left(u_{1}\right)\left(i^{\prime}, j^{\prime} \in \tilde{I}\right)$,

$\left(\mathcal{X} 3^{\varepsilon, \pm}\right) \sum_{\sigma \in \mathcal{S}_{3}} \sigma \cdot\left(\left(q^{-3 \varepsilon} u_{1}^{ \pm \varepsilon}-\left(q+q^{-1}\right) u_{2}^{ \pm \varepsilon}+q^{3 \varepsilon} u_{3}^{ \pm \varepsilon}\right) \mathcal{X}_{i^{\prime}}^{ \pm}\left(u_{1}\right) \mathcal{X}_{i^{\prime}}^{ \pm}\left(u_{2}\right) \mathcal{X}_{i^{\prime}}^{ \pm}\left(u_{3}\right)\right)=0$

$$
\begin{aligned}
& \sum_{\sigma \in \mathcal{S}_{1-a_{i j}}} \sigma \cdot \sum_{u=0}^{1-a_{i j}}(-1)^{u}\left[\begin{array}{c}
1-a_{i j} \\
u
\end{array}\right]_{q_{i}} \mathcal{X}_{i^{\prime}, p_{1}}^{ \pm} \cdot \ldots \cdot \mathcal{X}_{i^{\prime}, p_{u}}^{ \pm} \mathcal{X}_{j^{\prime}, v}^{ \pm} \mathcal{X}_{i^{\prime}, p_{u+1}}^{ \pm} \ldots \cdot \mathcal{X}_{i^{\prime}, p_{1-a_{i j}}}^{ \pm}=0 \\
& \left(k=1, i^{\prime}, j^{\prime} \in \tilde{I}, i^{\prime} \neq j^{\prime}\right), \\
& \left(\mathcal{X} \mathcal{P}^{ \pm}\right) \quad \sum_{\sigma \in \mathcal{S}_{2}} \sigma \cdot\left(P _ { i ^ { \prime } j ^ { \prime } } ^ { \pm } ( u _ { 1 } , u _ { 2 } ) \left(\mathcal{X}_{j^{\prime}}^{ \pm}(v) \mathcal{X}_{i^{\prime}}^{ \pm}\left(u_{1}\right) \mathcal{X}_{i^{\prime}}^{ \pm}\left(u_{2}\right)\right.\right. \\
& \begin{array}{r}
\left.\left.-[2]_{q^{m_{i^{\prime} j^{\prime}}}} \mathcal{X}_{i^{\prime}}^{ \pm}\left(u_{1}\right) \mathcal{X}_{j^{\prime}}^{ \pm}(v) \mathcal{X}_{i^{\prime}}^{ \pm}\left(u_{2}\right)+\mathcal{X}_{i^{\prime}}^{ \pm}\left(u_{1}\right) \mathcal{X}_{i^{\prime}}^{ \pm}\left(u_{2}\right) \mathcal{X}_{j^{\prime}}^{ \pm}(v)\right)\right)=0 \\
\left(k>1, i^{\prime}, j^{\prime} \in \tilde{I}, \chi\left(i^{\prime}\right) \neq j^{\prime}, \tilde{a}_{i^{\prime} j^{\prime}}<0\right),
\end{array}
\end{aligned}
$$

where $\tilde{\mathcal{H}}_{i^{\prime}, r}^{ \pm}, \tilde{b}_{i^{\prime} j^{\prime} r}, \mathcal{X}_{i^{\prime}}^{ \pm}(u), F_{i^{\prime} j^{\prime}}^{ \pm}\left(u_{1}, u_{2}\right), G_{i^{\prime} j^{\prime}}^{ \pm}\left(u_{1}, u_{2}\right), \varepsilon, P_{i^{\prime} j^{\prime}}^{ \pm}\left(u_{1}, u_{2}\right)$ and $m_{i^{\prime} j^{\prime}}$ are defined as follows:

$$
\sum_{r \in \mathbb{Z}} \tilde{\mathcal{H}}_{i^{\prime}, \pm r}^{ \pm} u^{r}=\exp \left( \pm\left(q-q^{-1}\right)\left[\frac{1}{2} \sum_{u \in \mathbb{Z} / k \mathbb{Z}}\left(\tilde{\alpha}_{\chi^{u}\left(i^{\prime}\right)} \mid \tilde{\alpha}_{i^{\prime}}\right)\right] \sum_{q>0} \mathcal{H}_{i^{\prime}, \pm r} u^{r}\right)
$$




$$
\begin{aligned}
& \tilde{b}_{i^{\prime} j^{\prime} r}=\frac{\sum_{u=0}^{k-1}\left[r\left(\tilde{\alpha}_{i^{\prime}} \mid \tilde{\alpha}_{\chi^{u}\left(j^{\prime}\right)}\right)\right]_{q} \omega^{r u}}{r\left[\frac{1}{2} \sum_{u \in \mathbb{Z} / k \mathbb{Z}}\left(\tilde{\alpha}_{i^{\prime}} \mid \tilde{\alpha}_{\chi^{u}\left(i^{\prime}\right)}\right)\right]_{q}} ; \\
& \mathcal{X}_{i^{\prime}}^{ \pm}(u)=\sum_{r \in \mathbb{Z}} \mathcal{X}_{i^{\prime}, r}^{ \pm} u^{-r} ; \\
& F_{i^{\prime} j^{\prime}}^{ \pm}\left(u_{1}, u_{2}\right)=\prod_{\substack{v \in \mathbb{Z} / k \mathbb{Z} \\
\tilde{a}_{i^{\prime}}, \chi^{v}\left(j^{\prime}\right) \neq 0}}\left(u_{1}-\omega^{v} q^{ \pm\left(\tilde{\alpha}_{i^{\prime}} \mid \tilde{\alpha}_{\chi^{v}\left(j^{\prime}\right)}\right)} u_{2}\right) ; \\
& G_{i^{\prime} j^{\prime}}^{ \pm}\left(u_{1}, u_{2}\right)=\prod_{\substack{v \in \mathbb{Z} / k \mathbb{Z} \\
\tilde{a}_{i^{\prime}}, \chi^{v}\left(j^{\prime}\right) \neq 0}}\left(q^{ \pm\left(\tilde{\alpha}_{i^{\prime}} \mid \tilde{\alpha}_{\chi^{v}\left(j^{\prime}\right)}\right)} u_{1}-\omega^{v} u_{2}\right) ; \\
& \varepsilon= \pm 1 \text {; } \\
& P_{i^{\prime} j^{\prime}}^{ \pm}\left(u_{1}, u_{2}\right)= \begin{cases}1 & \text { if } \tilde{a}_{i^{\prime}, \chi\left(i^{\prime}\right)}=0 \text { and } \chi\left(j^{\prime}\right) \neq j^{\prime}, \text { or } \chi\left(i^{\prime}\right)=i^{\prime} \\
\frac{q^{ \pm 2 k} u_{1}^{k}-u_{2}^{k}}{q^{ \pm 2} u_{1}-u_{2}} & \text { otherwise }\end{cases} \\
& m_{i^{\prime} j^{\prime}}= \begin{cases}\frac{k}{\tilde{d}} \sum_{u \in \mathbb{Z} / k \mathbb{Z}} \delta_{i^{\prime} \chi \chi^{u}\left(i^{\prime}\right)} & \text { if } \tilde{a}_{i^{\prime}, \chi\left(i^{\prime}\right)}=0 \text { and } \chi\left(j^{\prime}\right) \neq j^{\prime}, \text { or } \chi\left(i^{\prime}\right)=i^{\prime}, \\
k & \text { otherwise. }\end{cases}
\end{aligned}
$$

Remark 4.3. In [Dr1] not all the relations $\left(\mathcal{X} 3^{\varepsilon, \pm}\right)$ appear, but just $\left(\mathcal{X} 3^{1,+}\right)$ and $\left(\mathcal{X} 3^{-1,-}\right)$; the relations $\left(\mathcal{X} 3^{-1,+}\right)$ and $\left(\mathcal{X} 3^{1,-}\right)$ are introduced in [CP3] as consequences of $(\mathcal{Z})-\left(\mathcal{X} \mathcal{F} \mathcal{G}^{ \pm}\right),\left(\mathcal{X} 3^{1,+}\right),\left(\mathcal{X} 3^{-1,-}\right),\left(\mathcal{S}^{ \pm}\right),\left(\mathcal{X} \mathcal{P}^{ \pm}\right)$, since their use simplifies some calculations, making evident some symmetries (the stability of the relations under the antiautomorphism $\tilde{\Omega}$ and the automorphism $\tilde{\Theta})$. Here we use the relations $\left(\mathcal{X} 3^{\varepsilon, \pm}\right)$ for the same reasons of simplification (see Remarks 8.3 and 8.5), proving in Proposition 10.1 the equivalence stated in [CP3].

Remark 4.4. (i) For all $r \in \mathbb{Z}$, the algebra generated by $\left\{\mathcal{Y}_{i^{\prime}} \mid i^{\prime} \in \tilde{I}\right\}$ with relations $\left\{\mathcal{Y}_{\chi\left(i^{\prime}\right)}=\omega^{r} \mathcal{Y}_{i^{\prime}} \mid i^{\prime} \in \tilde{I}\right\}$ is isomorphic to the algebra generated by $\left\{Y_{i} \mid i \in I_{0}\right\}$ with relations $\left\{Y_{i}=\omega^{r \#\left\{i^{\prime} \in \tilde{I} \mid \bar{i}^{\prime}=i\right\}} Y_{i} \mid i \in I_{0}\right\}$, where a section $\sim I_{0} \rightarrow \tilde{I}$ induces an isomorphism $Y_{i} \mapsto \mathcal{Y}_{\hat{i}}$.

(ii) Consider $i^{\prime} \in \tilde{I}$ and let $i \in I_{0}=\tilde{I} / \chi$ be the $\chi$-orbit of $i^{\prime}$. Notice that $\chi\left(i^{\prime}\right)=i^{\prime}$ $\Leftrightarrow k \mid \tilde{d}_{i}$; more precisely $\sum_{u \in \mathbb{Z} / k \mathbb{Z}} \delta_{i^{\prime}, \chi^{u}\left(i^{\prime}\right)}=\tilde{d}_{i}$ and $\tilde{d}_{i} \#\left\{i^{\prime} \in \tilde{I} \mid \bar{i}^{\prime}=i\right\}=k$.

(iii) For all $r \in \mathbb{Z}$, the algebra generated by $\left\{Y_{i} \mid i \in I_{0}\right\}$ with relations $\left\{Y_{i}=\omega^{k r / \tilde{d}_{i}} Y_{i} \mid i \in I_{0}\right\}$ is trivially isomorphic to the algebra generated by $\left\{Y_{i} \mid i \in I_{0}\right\}$ with relations $\left\{Y_{i}=0 \mid \tilde{d}_{i} \nmid r\right\}$, which is trivially isomorphic to the free algebra generated by $\left\{Y_{i}\left|i \in I_{0}, \tilde{d}_{i}\right| r\right\}$.

(iv) Hence, for all $r \in \mathbb{Z}$, the algebra generated by $\left\{\mathcal{Y}_{i^{\prime}} \mid i^{\prime} \in \tilde{I}\right\}$ with relations $\left\{\mathcal{Y}_{\chi\left(i^{\prime}\right)}=\omega^{r} \mathcal{Y}_{i^{\prime}} \mid i^{\prime} \in \tilde{I}\right\}$ is isomorphic to the algebra generated by $\left\{Y_{i} \mid i \in I_{0}\right\}$ 
with relations $\left\{Y_{i}=0 \mid \tilde{d}_{i} \nmid r\right\}$, where a section $\sim: I_{0} \rightarrow \tilde{I}$ induces an isomorphism $Y_{i} \mapsto \mathcal{Y}_{\hat{i}}$.

(v) Finally, the algebra generated by $\left\{\mathcal{Y}_{i^{\prime}, r} \mid i^{\prime} \in \tilde{I}, r \in \mathbb{Z}\right\}$ with relations $\left\{\mathcal{Y}_{\chi\left(i^{\prime}\right), r}=\omega^{r} \mathcal{Y}_{i^{\prime}, r} \mid i^{\prime} \in \tilde{I}, r \in \mathbb{Z}\right\}$ is isomorphic to the algebra generated by $\left\{Y_{i, r} \mid i \in I_{0}, r \in \mathbb{Z}\right\}$ with relations $\left\{Y_{i, r}=0 \mid \tilde{d}_{i} \nmid r\right\}$, or equivalently to the free algebra generated by $\left\{Y_{i, r}\left|i \in I_{0}, r \in \mathbb{Z}, \tilde{d}_{i}\right| r\right\}$.

Notation 4.5. Set $I_{\mathbb{Z}}=\left\{(i, r) \in I_{0} \times \mathbb{Z}\left|\tilde{d}_{i}\right| r\right\}$.

Corollary 4.6. (i) $\mathcal{U}_{q}^{\mathrm{Dr}}$ is (isomorphic to) an algebra generated by

(G) $C^{ \pm 1}, \quad k_{i}^{ \pm 1}\left(i \in I_{0}\right), \quad X_{i, r}^{ \pm}\left((i, r) \in I_{0} \times \mathbb{Z}\right), \quad H_{i, r}\left((i, r) \in I_{0} \times(\mathbb{Z} \backslash\{0\})\right) ;$

the relations

$$
\begin{array}{ll}
X_{i, r}^{ \pm}=0 & \forall(i, r) \in\left(I_{0} \times \mathbb{Z}\right) \backslash I_{\mathbb{Z}}, \\
H_{i, r}=0 & \forall(i, r) \in\left(I_{0} \times \mathbb{Z}\right) \backslash I_{\mathbb{Z}}
\end{array}
$$

hold in $\mathcal{U}_{q}^{\mathrm{Dr}}$.

(ii) $\mathcal{U}_{q}^{\mathrm{Dr}}$ is generated by

$\left(G^{\prime}\right) C^{ \pm 1}, \quad k_{i}^{ \pm 1}\left(i \in I_{0}\right), \quad X_{i, r}^{ \pm}\left((i, r) \in I_{\mathbb{Z}}\right), \quad H_{i, r}\left((i, r) \in I_{\mathbb{Z}} \backslash\left(I_{0} \times\{0\}\right)\right)$.

Remark 4.7. The relations $\left(\mathcal{Z X}^{ \pm}\right)$are equivalent to the condition $\mathcal{X}_{\chi\left(i^{\prime}\right)}^{ \pm}(u)=$ $\mathcal{X}_{i^{\prime}}^{ \pm}\left(\omega^{-1} u\right)$ for all $i^{\prime} \in \tilde{I}$.

Notation 4.8. Given $i, j \in I_{0}$ we set $\tilde{d}_{i j}=\max \left\{\tilde{d}_{i}, \tilde{d}_{j}\right\}$.

Remark 4.9. (i) If $\tilde{\alpha}_{i^{\prime}}\left(i^{\prime} \in \tilde{I}\right)$ is a short root then $\left(\tilde{\alpha}_{i^{\prime}} \mid \tilde{\alpha}_{i^{\prime}}\right)=2 k / \tilde{d}$;

(ii) for all $i^{\prime} \in \tilde{I}$ we have $\sum_{u \in \mathbb{Z} / k \mathbb{Z}}\left(\tilde{\alpha}_{i^{\prime}} \mid \tilde{\alpha}_{\chi^{u}\left(i^{\prime}\right)}\right)=2 d_{\bar{i}^{\prime}}$.

Remark 4.10. (i) Note that there exists a section ${ }^{\sim}: I_{0} \rightarrow \tilde{I}$ such that given $i, j \in I_{0}$ we have $a_{i j} \neq 0 \Rightarrow \tilde{a}_{\tilde{i} \tilde{j}} \neq 0$ (of course it is always true that $\tilde{a}_{i^{\prime} j^{\prime}} \neq 0$ $\left.\Rightarrow a_{\bar{i}^{\prime} \bar{j}^{\prime}} \neq 0\right)$;

(ii) let $\sim$ be a section as in (i); then, if $k>1, d_{i} a_{i j}=\max \left\{d_{i}, d_{j}\right\} \tilde{a}_{\tilde{i} \tilde{j}}$.

Remark 4.11. (i) The relations $(\mathcal{K K}),\left(\mathcal{K} \mathcal{X}^{ \pm}\right)$and $(\mathcal{K H})$ are compatible with $(\mathcal{Z})$ and $\left(\mathcal{Z} \mathcal{X}^{ \pm}\right)$, in the sense that for all $i^{\prime}, j^{\prime} \in \tilde{I}, r \in \mathbb{Z}, s \in \mathbb{Z} \backslash\{0\}$,

$$
\begin{gathered}
(\mathcal{K K})_{\chi\left(i^{\prime}\right), j^{\prime}}=(\mathcal{K K})_{i^{\prime}, j^{\prime}}=(\mathcal{K K})_{i^{\prime}, \chi\left(j^{\prime}\right)}, \\
\left(\mathcal{K} \mathcal{X}^{ \pm}\right)_{\chi\left(i^{\prime}\right), j^{\prime}, r}=\left(\mathcal{K} \mathcal{X}^{ \pm}\right)_{i^{\prime}, j^{\prime}, r}, \quad\left(\mathcal{K} \mathcal{X}^{ \pm}\right)_{i^{\prime}, \chi\left(j^{\prime}\right), r}=\omega^{r}\left(\mathcal{K} \mathcal{X}^{ \pm}\right)_{i^{\prime}, j^{\prime}, r}, \\
(\mathcal{K H})_{\chi\left(i^{\prime}\right), j^{\prime}, s}=(\mathcal{K} \mathcal{H})_{i^{\prime}, j^{\prime}, s}, \quad(\mathcal{K H})_{i^{\prime}, \chi\left(j^{\prime}\right), s}=\omega^{s}(\mathcal{K H})_{i^{\prime}, j^{\prime}, s}
\end{gathered}
$$


(ii) if $i, j \in I_{0}$ are such that $\overline{i^{\prime}}=i, \overline{j^{\prime}}=j$, then $\left(\mathcal{K} \mathcal{X}^{ \pm}\right)_{i^{\prime}, j^{\prime}}$ is equivalent to

$$
\mathcal{K}_{i^{\prime}} \mathcal{X}_{j^{\prime}, r}^{ \pm}=q_{i}^{ \pm a_{i j}} \mathcal{X}_{j^{\prime}, r}^{ \pm} \mathcal{K}_{i^{\prime}}
$$

(see §1(II), Remark 4.9(ii) and Notation 3.1(i)).

Remark 4.12. (i) If we apply $\chi$ to the expression $\sum_{r>0} \mathcal{H}_{i^{\prime}, \pm r} u^{r}\left(i^{\prime} \in \tilde{I}\right)$ we get $($ see $(\mathcal{Z}))$

$$
\sum_{r>0} \mathcal{H}_{\chi\left(i^{\prime}\right), \pm r} u^{r}=\sum_{r>0} \mathcal{H}_{i^{\prime}, \pm r}\left(\omega^{ \pm 1} u\right)^{r}
$$

(ii) From (i) and from Definition 4.2 we get

$$
\sum_{r \in \mathbb{Z}} \tilde{\mathcal{H}}_{\chi\left(i^{\prime}\right), \pm r}^{ \pm} u^{r}=\sum_{r \in \mathbb{Z}} \tilde{\mathcal{H}}_{i^{\prime}, \pm r}^{ \pm}\left(\omega^{ \pm 1} u\right)^{r} \quad \forall i^{\prime} \in \tilde{I}
$$

that is, $\tilde{\mathcal{H}}_{\chi\left(i^{\prime}\right), r}^{ \pm}=\omega^{r} \tilde{\mathcal{H}}_{i^{\prime}, r}^{ \pm}$for all $\left(i^{\prime}, r\right) \in \tilde{I} \times(\mathbb{Z} \backslash\{0\})$.

(iii) The relations $(\mathcal{X X})$ are compatible with $(\mathcal{Z})$ and $\left(\mathcal{Z X}^{ \pm}\right)$:

$$
(\mathcal{X X})_{\chi\left(i^{\prime}\right), r ; j^{\prime}, s}=\omega^{r}(\mathcal{X X})_{i^{\prime}, r ; j^{\prime}, s}, \quad(\mathcal{X X})_{i^{\prime}, r ; \chi\left(j^{\prime}\right), s}=\omega^{s}(\mathcal{X} \mathcal{X})_{i^{\prime}, r ; j^{\prime}, s} .
$$

(iv) If $\sim$ is as in Remark 4.10 and $i, j \in I_{0}$, then $(\mathcal{X X})_{\tilde{i}, r ; \tilde{j}, s}$ is equivalent to

$$
\left[\mathcal{X}_{\tilde{i}, r}^{+}, \mathcal{X}_{\tilde{j}, s}^{-}\right]= \begin{cases}\delta_{i j} \frac{\mathcal{C}^{-s} \mathcal{K}_{\tilde{i}} \tilde{\mathcal{H}}_{\tilde{i}, r+s}^{+}-\mathcal{C}^{-r} \mathcal{K}_{\tilde{i}}^{-1} \tilde{\mathcal{H}}_{\tilde{i}, r+s}^{-}}{\left(q_{i}-q_{i}^{-1}\right)} & \text { if } \tilde{d}_{j} \mid s \\ 0 & \text { otherwise }\end{cases}
$$

(see Remark 4.4(ii)).

Remark 4.13. Let $i^{\prime}, j^{\prime} \in \tilde{I}, r \in \mathbb{Z}$; then:

(i) $\tilde{b}_{i^{\prime} j^{\prime} r}=\frac{1}{r} \sum_{u=0}^{k-1}\left[\frac{r\left(\tilde{\alpha}_{i^{\prime}} \mid \tilde{\alpha}_{\chi^{u}\left(j^{\prime}\right)}\right)}{d_{\bar{i}^{\prime}}}\right]_{q_{\bar{i}^{\prime}}} \omega^{r u}$;

(ii) $\tilde{b}_{\chi\left(i^{\prime}\right) j^{\prime} r}=\omega^{r} \tilde{b}_{i^{\prime} j^{\prime} r}$ and $\tilde{b}_{i^{\prime} \chi\left(j^{\prime}\right) r}=\omega^{-r} \tilde{b}_{i^{\prime} j^{\prime} r}$;

(iii) the relations $\left(\mathcal{H X}^{ \pm}\right)$and $(\mathcal{H H})$ are compatible with $(\mathcal{Z})$ and $\left(\mathcal{Z X}^{ \pm}\right)$:

$$
\begin{aligned}
\left(\mathcal{H} \mathcal{X}^{ \pm}\right)_{\chi\left(i^{\prime}\right), r ; j^{\prime}, s} & =\omega^{r}\left(\mathcal{H} \mathcal{X}^{ \pm}\right)_{i^{\prime}, r ; j^{\prime}, s}, & \left(\mathcal{H} \mathcal{X}^{ \pm}\right)_{i^{\prime}, r ; \chi\left(j^{\prime}\right), s} & =\omega^{s}\left(\mathcal{H} \mathcal{X}^{ \pm}\right)_{i^{\prime}, r ; j^{\prime}, s} \\
(\mathcal{H H})_{\chi\left(i^{\prime}\right), r ; j^{\prime}, s} & =\omega^{r}(\mathcal{H} \mathcal{H})_{i^{\prime}, r ; j^{\prime}, s}, & (\mathcal{H} \mathcal{H})_{i^{\prime}, r ; \chi\left(j^{\prime}\right), s} & =\omega^{s}(\mathcal{H H})_{i^{\prime}, r ; j^{\prime}, s}
\end{aligned}
$$

Notation 4.14. Let $i, j \in I_{0}$ and $r \in \mathbb{Z}$; then set

$$
b_{i j r}= \begin{cases}0 & \text { if } \tilde{d}_{i, j} \nmid r, \\ {[2 r]_{q}\left(q^{2 r}+(-1)^{r-1}+q^{-2 r}\right) / r} & \text { if }\left(X_{\tilde{n}}^{(k)}, i, j\right)=\left(A_{2 n}^{(2)}, 1,1\right), \\ {\left[\tilde{r} a_{i j}\right]_{q_{i}} / \tilde{r}} & \text { otherwise, with } \tilde{r}=r / \tilde{d}_{i, j} .\end{cases}
$$


Proposition 4.15. If $\sim$ is as in Remark 4.10, then $\tilde{b}_{\tilde{i} \tilde{j} r}=b_{i j r}$ for all $i, j \in I_{0}$ and $r \in \mathbb{Z}$. In particular $(\mathcal{H} \mathcal{X})_{\tilde{i}, r ; \tilde{j}, s}$ and $(\mathcal{H} \mathcal{H})_{\tilde{i}, r ; \tilde{j}, s}$ are equivalent to

$$
\left[\mathcal{H}_{\tilde{i}, r}, \mathcal{X}_{\tilde{j}, s}^{ \pm}\right]= \pm b_{i j r} \mathcal{C}^{\frac{r \mp|r|}{2}} \mathcal{X}_{\tilde{j}, r+s}^{ \pm} \quad \text { and } \quad\left[\mathcal{H}_{\tilde{i}, r}, \mathcal{H}_{\tilde{j}, s}\right]=\delta_{r+s, 0} b_{i j r} \frac{C^{r}-C^{-r}}{q_{j}-q_{j}^{-1}}
$$

Proof. If $k=1$ the claim is trivial. Suppose now $k>1$ (so that $X_{\tilde{n}}$ is simply laced) and notice that (see Remark 4.9(i))

$$
\tilde{b}_{\tilde{i} \tilde{j} r}=\frac{1}{r} \sum_{u=0}^{k-1}\left[\frac{r k \tilde{a}_{\tilde{i} \chi^{u}(\tilde{j})}}{\tilde{d} d_{i}}\right]_{q_{i}} \omega^{r u}
$$

moreover if $\tilde{d}_{i j}=k$ then either $\chi(\tilde{i})=\tilde{i}$ or $\chi(\tilde{j})=\tilde{j}$ so that $\tilde{a}_{\tilde{i} \chi^{u}(\tilde{j})}=\tilde{a}_{\tilde{i} \tilde{j}}$ for all $u$, $\tilde{r}=r / k, \tilde{d}_{h}=d_{h}$ for all $h \in I_{0}, \tilde{d}=k$ and, thanks to Remark 4.10(ii),

$$
\tilde{b}_{\tilde{i} \tilde{j} r}=\frac{1}{r}\left[\frac{r k \tilde{a}_{\tilde{i} \tilde{j}}}{\tilde{d} d_{i}}\right]_{q_{i}} \sum_{u=0}^{k-1} \omega^{r u}= \begin{cases}0 & \text { if } k \nmid r, \\ \frac{1}{\tilde{r}}\left[\frac{r a_{i j}}{\tilde{d}}\right]_{q_{i}}=\frac{\left[\tilde{r} a_{i j}\right]_{q_{i}}}{\tilde{r}} & \text { if } k \mid r .\end{cases}
$$

If $\left(X_{\tilde{n}}^{(k)}, i, j\right)=\left(A_{2 n}^{(2)}, 1,1\right)$ then

$$
\tilde{b}_{\tilde{i} \tilde{j} r}=\frac{1}{r}\left([4 r]_{q}+(-1)^{r}[-2 r]_{q}\right)=\frac{1}{r}[2 r]_{q}\left(q^{2 r}+(-1)^{r-1}+q^{-2 r}\right) .
$$

In the remaining cases $\tilde{a}_{\tilde{i} \chi^{u}(\tilde{j})}=0$ when $k \nmid u, \tilde{r}=r$ and $\max \left\{d_{i}, d_{j}\right\}=k / \tilde{d}$, hence

$$
\tilde{b}_{\tilde{i} \tilde{j} r}=\frac{1}{r}\left[\frac{k r \tilde{a}_{\tilde{i} \tilde{j}}}{\tilde{d} d_{i}}\right]_{q_{i}}=\frac{1}{r}\left[r a_{i j}\right]_{q_{i}}=\frac{1}{\tilde{r}}\left[\tilde{r} a_{i j}\right]_{q_{i}} .
$$

In the next remarks as well as in all the paper the $q$-commutators play a fundamental role in simplifying the description of the elements and in the computations. We recall here their definition and simple properties (see also [Jn]).

Notation 4.16. Given $v \in \mathbb{C}(q) \backslash\{0\}$ and $a, b$ elements of a $\mathbb{C}(q)$-algebra, the element $[a, b]_{v}$ is defined by $[a, b]_{v}=a b-v b a$.

Remark 4.17. Let $a, b, c \in \tilde{\mathcal{U}}_{q}^{\mathrm{Dr}}$ and $u, v, w \in \mathbb{C}(q) \backslash\{0\}$. Then:

(i) $[a, b]_{u}=-u[b, a]_{u^{-1}}$;

(ii) $\left[[a, b]_{u}, b\right]_{v}=\left[[a, b]_{v}, b\right]_{u}=a b^{2}-(u+v) b a b+u v b^{2} a$;

(iii) $\left[[a, b]_{u}, c\right]_{v}=\left[a,[b, c]_{v / w}\right]_{u w}-u\left[b,[a, c]_{w}\right]_{v / u w}$.

If moreover $a \in \mathcal{U}_{q, \alpha}^{\mathrm{Dr}}, b \in \mathcal{U}_{q, \beta}^{\mathrm{Dr}}$ and $i \in I_{0}$ then:

(iv) $\left[k_{i} a, b\right]_{u}=k_{i}[a, b]_{q^{-\left(\alpha_{i} \mid \beta\right)} u}$; 
(v) $\left[a, k_{i} b\right]_{u}=q^{-\left(\alpha \mid \alpha_{i}\right)} k_{i}[a, b]_{q^{\left(\alpha_{i} \mid \alpha\right)} u}$.

Remark 4.18. Let $i^{\prime}, j^{\prime} \in \tilde{I}$; then:

(i) $F_{i^{\prime} j^{\prime}}^{ \pm}\left(u_{1}, u_{2}\right)$ and $G_{i^{\prime} j^{\prime}}^{ \pm}\left(u_{1}, u_{2}\right)$ are homogeneous polynomials of the same degree $d$;

(ii) $F_{\chi\left(i^{\prime}\right) j^{\prime}}^{ \pm}\left(u_{1}, u_{2}\right)=\omega^{d} F_{i^{\prime} j^{\prime}}^{ \pm}\left(\omega^{-1} u_{1}, u_{2}\right), F_{i^{\prime} \chi\left(j^{\prime}\right)}^{ \pm}\left(u_{1}, u_{2}\right)=F_{i^{\prime} j^{\prime}}^{ \pm}\left(u_{1}, \omega^{-1} u_{2}\right)$;

(iii) $G_{\chi\left(i^{\prime}\right) j^{\prime}}^{ \pm}\left(u_{1}, u_{2}\right)=\omega^{d} G_{i^{\prime} j^{\prime}}^{ \pm}\left(\omega^{-1} u_{1}, u_{2}\right), G_{i^{\prime} \chi\left(j^{\prime}\right)}^{ \pm}\left(u_{1}, u_{2}\right)=G_{i^{\prime} j^{\prime}}^{ \pm}\left(u_{1}, \omega^{-1} u_{2}\right)$;

(iv) the relations $\left(\mathcal{X} \mathcal{F G}^{ \pm}\right)$are compatible with $\left(\mathcal{Z X}^{ \pm}\right)$:

$$
\begin{aligned}
& \left(\mathcal{X} \mathcal{F} \mathcal{G}^{ \pm}\right)_{\chi\left(i^{\prime}\right), j^{\prime}}\left(u_{1}, u_{2}\right)=\omega^{d}\left(\mathcal{X} \mathcal{F} \mathcal{G}^{ \pm}\right)_{i^{\prime}, j^{\prime}}\left(\omega^{-1} u_{1}, u_{2}\right), \\
& \left(\mathcal{X} \mathcal{F} \mathcal{G}^{ \pm}\right)_{i^{\prime}, \chi\left(j^{\prime}\right)}\left(u_{1}, u_{2}\right)=\left(\mathcal{X} \mathcal{F} \mathcal{G}^{ \pm}\right)_{i^{\prime}, j^{\prime}}\left(u_{1}, \omega^{-1} u_{2}\right) .
\end{aligned}
$$

Remark 4.19. Let $i^{\prime}, j^{\prime} \in \tilde{I}$ be such that $\tilde{a}_{i^{\prime}, \chi^{r}\left(j^{\prime}\right)}=0$ for all $r \in \mathbb{Z}$; this is equivalent to the condition $a_{\bar{i}^{\prime} \bar{j}^{\prime}}=0$. Then:

(i) $F_{i^{\prime} j^{\prime}}^{ \pm}\left(u_{1}, u_{2}\right)=G_{i^{\prime} j^{\prime}}^{ \pm}\left(u_{1}, u_{2}\right)=1$;

(ii) the relation $\left(\mathcal{X} \mathcal{F} \mathcal{G}^{ \pm}\right)_{i^{\prime}, j^{\prime}}$ is equivalent to $\left[\mathcal{X}_{i^{\prime}}^{ \pm}\left(u_{1}\right), \mathcal{X}_{j^{\prime}}^{ \pm}\left(u_{2}\right)\right]=0$, that is, to

$$
\left[\mathcal{X}_{i^{\prime}, r}^{ \pm}, \mathcal{X}_{j^{\prime}, s}^{ \pm}\right]=0 \quad \forall r, s \in \mathbb{Z} .
$$

Remark 4.20. Let $i^{\prime}, j^{\prime} \in \tilde{I}$.

(i) The condition $\left(\tilde{\alpha}_{i^{\prime}} \mid \tilde{\alpha}_{\chi^{r}\left(j^{\prime}\right)}\right)=\left(\tilde{\alpha}_{i^{\prime}} \mid \tilde{\alpha}_{j^{\prime}}\right) \neq 0$ for all $r \in \mathbb{Z}$ is equivalent to the

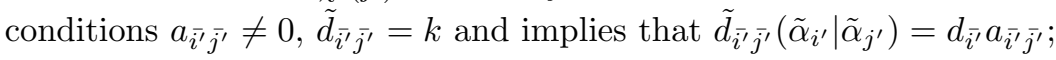

(ii) the condition that there is a unique $r \in \mathbb{Z} / k \mathbb{Z}$ such that $\left(\tilde{\alpha}_{i^{\prime}} \mid \tilde{\alpha}_{\chi^{r}\left(j^{\prime}\right)}\right) \neq 0$ is equivalent to the conditions $a_{\bar{i}^{\prime} \bar{j}^{\prime}} \neq 0, \tilde{d}_{\bar{i}^{\prime} \bar{j}^{\prime}}=1,\left(X_{\tilde{n}}^{(k)},{\overline{i^{\prime}}}^{\prime}, \bar{j}^{\prime}\right) \neq\left(A_{2 n}^{(2)}, 1,1\right)$; this condition implies that $\left(\tilde{\alpha}_{i^{\prime}} \mid \tilde{\alpha}_{\chi^{r}\left(j^{\prime}\right)}\right)=d_{{\overline{i^{\prime}}}^{\prime}} a_{\bar{i}^{\prime} j^{\prime}}$.

Let $i, j \in I_{0}$ and choose ${ }^{\sim}$ as in Remark 4.10 .

(iii) If $a_{i j} \neq 0$ and $\left(X_{\tilde{n}}^{(k)}, i, j\right) \neq\left(A_{2 n}^{(2)}, 1,1\right)$ (that is, $\tilde{i}, \tilde{j}$ satisfy the conditions of (i) or (ii) with $r=0$ ) then

$$
F_{\tilde{i} \tilde{j}}^{ \pm}\left(u_{1}, u_{2}\right)=u_{1}^{\tilde{d}_{i j}}-q_{i}^{ \pm a_{i j}} u_{2}^{\tilde{d}_{i j}}, \quad G_{\tilde{i} \tilde{j}}^{ \pm}\left(u_{1}, u_{2}\right)=q_{i}^{ \pm a_{i j}} u_{1}^{\tilde{d}_{i j}}-u_{2}^{\tilde{d}_{i j}},
$$

and the relation $\left(\mathcal{X F \mathcal { G } ^ { \pm }}\right)_{\tilde{i}, \tilde{j}}$ is equivalent to

$$
\left[u_{1}^{\tilde{d}_{i j}} \mathcal{X}_{\tilde{i}}^{ \pm}\left(u_{1}\right), \mathcal{X}_{\tilde{j}}^{ \pm}\left(u_{2}\right)\right]_{q_{i}^{ \pm a_{i j}}}+\left[u_{2}^{\tilde{d}_{i j}} \mathcal{X}_{\tilde{j}}^{ \pm}\left(u_{2}\right), \mathcal{X}_{\tilde{i}}^{ \pm}\left(u_{1}\right)\right]_{q_{i}^{ \pm a_{i j}}}=0,
$$

that is, to

$$
\left[\mathcal{X}_{\tilde{i}, r+\tilde{d}_{i j}}^{ \pm}, \mathcal{X}_{\tilde{j}, s}^{ \pm}\right]_{q_{i} \pm a_{i j}}+\left[\mathcal{X}_{\tilde{j}, s+\tilde{d}_{i j}}^{ \pm}, \mathcal{X}_{\tilde{i}, r}^{ \pm}\right]_{q_{i}^{ \pm a_{i j}}}=0 \quad \forall r, s \in \mathbb{Z} .
$$


Notice that

$$
\left[\mathcal{X}_{\tilde{i}, r+\tilde{d}_{i j}}^{-}, \mathcal{X}_{\tilde{j}, s}^{-}\right]_{q_{i}^{-a_{i j}}}=-q_{i}^{-a_{i j}}\left[\mathcal{X}_{\tilde{j},\left(s+\tilde{d}_{i j}\right)-\tilde{d}_{i j}}^{-}, \mathcal{X}_{\tilde{i}, r+\tilde{d}_{i j}}^{-}\right]_{q_{i}{ }^{a_{i j}}}
$$

so that $\left(\mathcal{X} \mathcal{F} \mathcal{G}^{ \pm}\right)_{\tilde{i}, \tilde{j}}$ is equivalent to

$$
\left[\mathcal{X}_{\tilde{i}, r \pm \tilde{d}_{i j}}^{ \pm}, \mathcal{X}_{\tilde{j}, s}^{ \pm}\right]_{q_{i} a_{i j}}+\left[\mathcal{X}_{\tilde{j}, s \pm \tilde{d}_{i j}}^{ \pm}, \mathcal{X}_{\tilde{i}, r}^{ \pm}\right]_{q_{i} a_{i j}}=0 \quad \forall r, s \in \mathbb{Z}
$$

Remark 4.21. Let $\left(X_{\tilde{n}}^{(k)}, i, j\right)=\left(A_{2 n}^{(2)}, 1,1\right)$; then if is as in Remark 4.10:

(i) $F_{\tilde{i} \tilde{j}}^{ \pm}\left(u_{1}, u_{2}\right)=\left(u_{1}-q^{ \pm 4} u_{2}\right)\left(u_{1}+q^{\mp 2} u_{2}\right)=u_{1}^{2}-\left(q^{ \pm 4}-q^{\mp 2}\right) u_{1} u_{2}-q^{ \pm 2} u_{2}^{2}$, $G_{\tilde{i} \tilde{j}}^{ \pm}\left(u_{1}, u_{2}\right)=q^{ \pm 2} u_{1}^{2}-\left(q^{ \pm 4}-q^{\mp 2}\right) u_{1} u_{2}-u_{2}^{2}$

(ii) the relation $\left(\mathcal{X} \mathcal{F} \mathcal{G}^{ \pm}\right)_{\tilde{i} \tilde{j}}$ is equivalent to

$$
\begin{aligned}
& {\left[u_{1}^{2} \mathcal{X}_{\tilde{1}}^{ \pm}\left(u_{1}\right), \mathcal{X}_{\tilde{1}}^{ \pm}\left(u_{2}\right)\right]_{q^{ \pm 2}}+\left[u_{2}^{2} \mathcal{X}_{\tilde{1}}^{ \pm}\left(u_{2}\right), \mathcal{X}_{\tilde{1}}^{ \pm}\left(u_{1}\right)\right]_{q^{ \pm 2}}} \\
& \quad-\left(q^{ \pm 4}-q^{\mp 2}\right)\left(u_{1} \mathcal{X}_{\tilde{1}}^{ \pm}\left(u_{1}\right) u_{2} \mathcal{X}_{\tilde{1}}^{ \pm}\left(u_{2}\right)+u_{2} \mathcal{X}_{\tilde{1}}^{ \pm}\left(u_{2}\right) u_{1} \mathcal{X}_{\tilde{1}}^{ \pm}\left(u_{1}\right)\right)=0
\end{aligned}
$$

that is, to

$$
\begin{aligned}
{\left[\mathcal{X}_{\tilde{1}, r+2}^{ \pm},\right.} & \left.\mathcal{X}_{\tilde{1}, s}^{ \pm}\right]_{q^{ \pm 2}}-q^{ \pm 4}\left[\mathcal{X}_{\tilde{1}, r+1}^{ \pm}, \mathcal{X}_{\tilde{1}, s+1}^{ \pm}\right]_{q^{\mp 6}} \\
& +\left[\mathcal{X}_{\tilde{1}, s+2}^{ \pm}, \mathcal{X}_{\tilde{1}, r}^{ \pm}\right]_{q^{ \pm 2}}-q^{ \pm 4}\left[\mathcal{X}_{\tilde{1}, s+1}^{ \pm}, \mathcal{X}_{\tilde{1}, r+1}^{ \pm}\right]_{q^{\mp 6}}=0 \quad \forall r, s \in \mathbb{Z} .
\end{aligned}
$$

As in Remark 4.20 notice that in this case $\left(\mathcal{X F} \mathcal{G}^{ \pm}\right)_{\tilde{i} \tilde{j}}$ is equivalent to

$$
\begin{aligned}
& {\left[\mathcal{X}_{\tilde{1}, r \pm 2}^{ \pm}, \mathcal{X}_{\tilde{1}, s}^{ \pm}\right]_{q^{2}}-q^{4}\left[\mathcal{X}_{\tilde{1}, r \pm 1}^{ \pm}, \mathcal{X}_{\tilde{1}, s \pm 1}^{ \pm}\right]_{q^{-6}}} \\
& \quad+\left[\mathcal{X}_{\tilde{1}, s \pm 2}^{ \pm}, \mathcal{X}_{\tilde{1}, r}^{ \pm}\right]_{q^{2}}-q^{4}\left[\mathcal{X}_{\tilde{1}, s \pm 1}^{ \pm}, \mathcal{X}_{\tilde{1}, r \pm 1}^{ \pm}\right]_{q^{-6}}=0 \quad \forall r, s \in \mathbb{Z} .
\end{aligned}
$$

Remark 4.22. Let $i^{\prime} \in \tilde{I}$; then

(i) the relations $\left(\mathcal{X} 3^{\varepsilon, \pm}\right)$ are compatible with $\left(\mathcal{Z} \mathcal{X}^{ \pm}\right)$:

$$
\left(\mathcal{X} 3^{\varepsilon, \pm}\right)_{\chi\left(i^{\prime}\right)}\left(u_{1}, u_{2}, u_{3}\right)=\left(\mathcal{X} 3^{\varepsilon, \pm}\right)_{i^{\prime}}\left(\omega^{-1} u_{1}, \omega^{-1} u_{2}, \omega^{-1} u_{3}\right)
$$

(ii) the condition $\tilde{a}_{\chi\left(i^{\prime}\right) i^{\prime}}=-1$ is equivalent to $\left(X_{\tilde{n}}^{(k)}, \bar{i}^{\prime}\right)=\left(A_{2 n}^{(k)}, 1\right)$;

(iii) the relations $\left(\mathcal{X} 3^{\varepsilon, \pm}\right)$ are equivalent to

$$
\begin{aligned}
\sum_{\sigma \in \mathcal{S}_{3}} \sigma \cdot\left(q^{-3 \varepsilon} u_{1}^{ \pm \varepsilon} \mathcal{X}_{i^{\prime}}^{ \pm}\left(u_{1}\right) \mathcal{X}_{i^{\prime}}^{ \pm}\left(u_{2}\right) \mathcal{X}_{i^{\prime}}^{ \pm}\left(u_{3}\right)-q^{-\varepsilon} \mathcal{X}_{i^{\prime}}^{ \pm}\left(u_{2}\right) u_{1}^{ \pm \varepsilon} \mathcal{X}_{i^{\prime}}^{ \pm}\left(u_{1}\right) \mathcal{X}_{i^{\prime}}^{ \pm}\left(u_{3}\right)\right. \\
\left.-q^{\varepsilon} \mathcal{X}_{i^{\prime}}^{ \pm}\left(u_{3}\right) u_{1}^{ \pm \varepsilon} \mathcal{X}_{i^{\prime}}^{ \pm}\left(u_{1}\right) \mathcal{X}_{i^{\prime}}^{ \pm}\left(u_{2}\right)+q^{3 \varepsilon} \mathcal{X}_{i^{\prime}}^{ \pm}\left(u_{3}\right) \mathcal{X}_{i^{\prime}}^{ \pm}\left(u_{2}\right) u_{1}^{ \pm \varepsilon} \mathcal{X}_{i^{\prime}}^{ \pm}\left(u_{1}\right)\right)=0
\end{aligned}
$$

which is

$$
q^{-3 \varepsilon} \sum_{\sigma \in \mathcal{S}_{3}} \sigma \cdot\left[\left[u_{1}^{ \pm \varepsilon} \mathcal{X}_{i^{\prime}}^{ \pm}\left(u_{1}\right), \mathcal{X}_{i^{\prime}}^{ \pm}\left(u_{2}\right)\right]_{q^{2 \varepsilon}}, \mathcal{X}_{i^{\prime}}^{ \pm}\left(u_{3}\right)\right]_{q^{4 \varepsilon}}=0
$$


or equivalently

$$
\sum_{\sigma \in \mathcal{S}_{3}} \sigma \cdot\left[\left[\mathcal{X}_{i^{\prime}, r_{1} \pm \varepsilon}^{ \pm}, \mathcal{X}_{i^{\prime}, r_{2}}^{ \pm}\right]_{q^{2 \varepsilon}}, \mathcal{X}_{i^{\prime}, r_{3}}^{ \pm}\right]_{q^{4 \varepsilon}}=0 \quad \forall r_{1}, r_{2}, r_{3} \in \mathbb{Z}
$$

Remark 4.23. The relations $\left(\mathcal{S}^{ \pm}\right)$are compatible with $\left(\mathcal{Z} \mathcal{X}^{ \pm}\right)$.

Remark 4.24. Let $k>1, i^{\prime}, j^{\prime} \in \tilde{I}$ be such that $\chi\left(i^{\prime}\right) \neq j^{\prime}, \tilde{a}_{i^{\prime} j^{\prime}}<0$ (this is equivalent to $\left.k>1, a_{\bar{i}^{\prime} \bar{j}^{\prime}}<0\right)$. It is immediate to see that:

(i) $P_{\chi\left(i^{\prime}\right) j^{\prime}}^{ \pm}\left(u_{1}, u_{2}\right)=P_{i^{\prime} j^{\prime}}^{ \pm}\left(u_{1}, u_{2}\right)=P_{i^{\prime} \chi\left(j^{\prime}\right)}^{ \pm}\left(u_{1}, u_{2}\right)$;

(ii) $m_{\chi\left(i^{\prime}\right) j^{\prime}}=m_{i^{\prime} j^{\prime}}=m_{i^{\prime} \chi\left(j^{\prime}\right)}$;

(iii) $P_{i^{\prime} j^{\prime}}^{ \pm}\left(u_{1}, u_{2}\right)$ is homogeneous (of some degree $d$ );

(iv) the relations $\left(\mathcal{X} \mathcal{P}^{ \pm}\right)$are compatible with $\left(\mathcal{Z} \mathcal{X}^{ \pm}\right)$:

$$
\begin{aligned}
\left(\mathcal{X} \mathcal{P}^{ \pm}\right)_{\chi\left(i^{\prime}\right), j^{\prime}}\left(u_{1}, u_{2} ; v\right) & =\omega^{d}\left(\mathcal{X} \mathcal{P}^{ \pm}\right)_{i^{\prime}, j^{\prime}}\left(\omega^{-1} u_{1}, \omega^{-1} u_{2} ; v\right) \\
\left(\mathcal{X}^{ \pm}\right)_{\chi\left(i^{\prime}\right), j^{\prime}}\left(u_{1}, u_{2} ; \omega^{-1} v\right) & =\left(\mathcal{X P}^{ \pm}\right)_{i^{\prime}, j^{\prime}}\left(u_{1}, u_{2} ; v\right)
\end{aligned}
$$

Moreover if $i, j \in I_{0}$ are such that $i=\bar{i}^{\prime}, j=\bar{j}^{\prime}$ then:

(v) the condition $\tilde{a}_{i^{\prime}, \chi\left(i^{\prime}\right)}=0$ and $\chi\left(j^{\prime}\right) \neq j^{\prime}$, or $\chi\left(i^{\prime}\right)=i^{\prime}$ is equivalent to $a_{i j}=-1$;

(vi) $m_{i^{\prime} j^{\prime}}= \begin{cases}d_{i} & \text { if } a_{i j}=-1, \\ k\left(=-a_{i j}\right) & \text { otherwise; }\end{cases}$

(vii) the relation $\left(\mathcal{X} \mathcal{P}^{ \pm}\right)_{i^{\prime}, j^{\prime}}$ is equivalent to

$$
\begin{aligned}
\sum_{\sigma \in \mathcal{S}_{2}} \sigma \cdot & \sum_{\substack{r, s \geq 0 \\
r+s=-1}} q^{ \pm 2 s}\left(\mathcal{X}_{j^{\prime}}^{ \pm}(v) u_{1}^{s} \mathcal{X}_{i^{\prime}}^{ \pm}\left(u_{1}\right) u_{2}^{r} \mathcal{X}_{i^{\prime}}^{ \pm}\left(u_{2}\right)\right. \\
& \left.-[2]_{q}{ }_{m_{i^{\prime} j^{\prime}}} u_{1}^{s} \mathcal{X}_{i^{\prime}}^{ \pm}\left(u_{1}\right) \mathcal{X}_{j^{\prime}}^{ \pm}(v) u_{2}^{r} \mathcal{X}_{i^{\prime}}^{ \pm}\left(u_{2}\right)+u_{1}^{s} \mathcal{X}_{i^{\prime}}^{ \pm}\left(u_{1}\right) u_{2}^{r} \mathcal{X}_{i^{\prime}}^{ \pm}\left(u_{2}\right) \mathcal{X}_{j^{\prime}}^{ \pm}(v)\right)=0
\end{aligned}
$$

that is,

$$
\begin{aligned}
\sum_{\sigma \in \mathcal{S}_{2}} \sigma . & \sum_{\substack{r, s \geq 0 \\
r+s=-1-a_{i j}}} q^{ \pm 2 s}\left(\mathcal{X}_{j^{\prime}, v}^{ \pm} \mathcal{X}_{i^{\prime}, p_{1}+s}^{ \pm} \mathcal{X}_{i^{\prime}, p_{2}+r}^{ \pm}\right. \\
& \left.-[2]_{q^{m_{i^{\prime} j^{\prime}}}} \mathcal{X}_{i^{\prime}, p_{1}+s}^{ \pm} \mathcal{X}_{j^{\prime}, v}^{ \pm} \mathcal{X}_{i^{\prime}, p_{2}+r}^{ \pm}+\mathcal{X}_{i^{\prime}, p_{1}+s}^{ \pm} \mathcal{X}_{i^{\prime}, p_{2}+r}^{ \pm} \mathcal{X}_{j^{\prime}, v}^{ \pm}\right)=0
\end{aligned}
$$

or equivalently

$$
\begin{aligned}
\sum_{\sigma \in \mathcal{S}_{2}} \sigma \cdot \sum_{\substack{r, s \geq 0 \\
r+s=-1-a_{i j}}} q^{2 s}\left(\mathcal{X}_{j^{\prime}, v}^{ \pm} \mathcal{X}_{i^{\prime}, p_{1} \pm s}^{ \pm} \mathcal{X}_{i^{\prime}, p_{2} \pm r}^{ \pm}\right. & \\
& \left.-[2]_{q^{m_{i^{\prime} j^{\prime}}}} \mathcal{X}_{i^{\prime}, p_{1} \pm s}^{ \pm} \mathcal{X}_{j^{\prime}, v}^{ \pm} \mathcal{X}_{i^{\prime}, p_{2} \pm r}^{ \pm}+\mathcal{X}_{i^{\prime}, p_{1} \pm s}^{ \pm} \mathcal{X}_{i^{\prime}, p_{2} \pm r}^{ \pm} \mathcal{X}_{j^{\prime}, v}^{ \pm}\right)=0
\end{aligned}
$$


We are now ready to write down an equivalent definition of $\mathcal{U}_{q}^{\operatorname{Dr}}\left(X_{\tilde{n}}^{(k)}\right)$, using the generators $(G)$.

Proposition 4.25. $\mathcal{U}_{q}^{\mathrm{Dr}}\left(X_{\tilde{n}}^{(k)}\right)$ is (isomorphic to) the $\mathbb{C}(q)$-algebra generated by $(G) \quad C^{ \pm 1}, \quad k_{i}^{ \pm 1}\left(i \in I_{0}\right), \quad X_{i, r}^{ \pm}\left((i, r) \in I_{0} \times \mathbb{Z}\right), \quad H_{i, r}\left((i, r) \in I_{0} \times(\mathbb{Z} \backslash\{0\})\right)$, with the following relations $(D R)$ :

$$
\begin{array}{ll}
X_{i, r}^{ \pm}=0 & \forall(i, r) \in\left(I_{0} \times \mathbb{Z}\right) \backslash I_{\mathbb{Z}}, \\
H_{i, r}=0 & \forall(i, r) \in\left(I_{0} \times \mathbb{Z}\right) \backslash I_{\mathbb{Z}},
\end{array}
$$

$$
[C, x]=0 \quad \forall x, \quad k_{i} k_{j}=k_{j} k_{i} \quad\left(i, j \in I_{0}\right),
$$$$
C C^{-1}=1, \quad k_{i} k_{i}^{-1}=1=k_{i}^{-1} k_{i} \quad\left(i \in I_{0}\right),
$$$$
k_{i} X_{j, r}^{ \pm}=q_{i}^{ \pm a_{i j}} X_{j, r}^{ \pm} k_{i} \quad\left(i \in I_{0},(j, r) \in I_{0} \times \mathbb{Z}\right),
$$

$$
\left[k_{i}, H_{j, s}\right]=0 \quad\left(i \in I_{0},(j, s) \in I_{0} \times(\mathbb{Z} \backslash\{0\})\right),
$$

$$
\left[X_{i, r}^{+}, X_{j, s}^{-}\right]= \begin{cases}\delta_{i, j} \frac{C^{-s} k_{i} \tilde{H}_{i, r+s}^{+}-C^{-r} k_{i}^{-1} \tilde{H}_{i, r+s}^{-}}{q_{i}-q_{i}^{-1}} & \text { if } \tilde{d}_{j} \mid s \\ 0 & \text { otherwise }\end{cases}
$$

$\left((i, r),(j, s) \in I_{0} \times \mathbb{Z}\right)$,

$\left(H X^{ \pm}\right)\left[H_{i, r}, X_{j, s}^{ \pm}\right]= \pm b_{i j r} C^{\frac{r \mp|r|}{2}} X_{j, r+s}^{ \pm} \quad\left((i, r) \in I_{0} \times(\mathbb{Z} \backslash\{0\}),(j, s) \in I_{0} \times \mathbb{Z}\right)$,

$$
\left[H_{i, r}, H_{j, s}\right]=\delta_{r+s, 0} b_{i j r} \frac{C^{r}-C^{-r}}{q_{j}-q_{j}^{-1}} \quad\left((i, r),(j, s) \in I_{0} \times(\mathbb{Z} \backslash\{0\})\right),
$$

$\left(X D^{ \pm}\right) \quad\left[X_{i, r \pm \tilde{d}_{i j}}^{ \pm}, X_{j, s}^{ \pm}\right]_{q_{i} a_{i j}}+\left[X_{j, s \pm \tilde{d}_{i j}}^{ \pm}, X_{i, r}^{ \pm}\right]_{q_{j}{ }^{a_{j i}}}=0 \quad\left((i, r),(j, s) \in I_{0} \times \mathbb{Z}, a_{i j}<0\right)$,

$\left(X 1^{ \pm}\right) \sum_{\sigma \in \mathcal{S}_{2}} \sigma \cdot\left[X_{i, r_{1} \pm \tilde{d}_{i}}^{ \pm}, X_{i, r_{2}}^{ \pm}\right]_{q_{i}^{2}}=0 \quad\left(\left(r_{1}, r_{2}\right) \in \mathbb{Z}^{2},\left(X_{\tilde{n}}^{(k)}, i\right) \neq\left(A_{2 n}^{(2)}, 1\right)\right)$,

$$
\sum_{\sigma \in \mathcal{S}_{2}} \sigma \cdot\left(\left[X_{1, r_{1} \pm 2}^{ \pm}, X_{1, r_{2}}^{ \pm}\right]_{q^{2}}-q^{4}\left[X_{1, r_{1} \pm 1}^{ \pm}, X_{1, r_{2} \pm 1}^{ \pm}\right]_{q^{-6}}\right)=0
$$

$$
\left(\left(r_{1}, r_{2}\right) \in \mathbb{Z}^{2}, X_{\tilde{n}}^{(k)}=A_{2 n}^{(2)}\right),
$$

$\left(X 3^{\varepsilon, \pm}\right) \quad \sum_{\sigma \in \mathcal{S}_{3}} \sigma \cdot\left[\left[X_{1, r_{1} \pm \varepsilon}^{ \pm}, X_{1, r_{2}}^{ \pm}\right]_{q^{2 \varepsilon}}, X_{1, r_{3}}^{ \pm}\right]_{q^{4 \varepsilon}}=0 \quad\left(\left(r_{1}, r_{2}, r_{3}\right) \in \mathbb{Z}^{3}, X_{\tilde{n}}^{(k)}=A_{2 n}^{(2)}\right)$,

$\left(S U L^{ \pm}\right) \sum_{\sigma \in \mathcal{S}_{1-a_{i j}}} \sigma \cdot \sum_{u=0}^{1-a_{i j}}(-1)^{u}\left[\begin{array}{c}1-a_{i j} \\ u\end{array}\right]_{q_{i}} X_{i, r_{1}}^{ \pm} \cdot \ldots \cdot X_{i, r_{u}}^{ \pm} X_{j, s}^{ \pm} X_{i, r_{u+1}}^{ \pm} \cdots \cdot X_{i, r_{1-a_{i j}}}^{ \pm}=0$ $\left(i \neq j \in I_{0}, a_{i j} \in\{0,-1\}\right.$ if $\left.k \neq 1, r=\left(r_{1}, \ldots, r_{1-a_{i j}}\right) \in \mathbb{Z}^{1-a_{i j}}, s \in \mathbb{Z}\right)$,

$$
\begin{array}{r}
\sum_{\sigma \in \mathcal{S}_{2}} \sigma \cdot\left(q\left(X_{j, s}^{ \pm} X_{i, r_{1} \pm 1}^{ \pm} X_{i, r_{2}}^{ \pm}-[2]_{q^{2}} X_{i, r_{1} \pm 1}^{ \pm} X_{j, s}^{ \pm} X_{i, r_{2}}^{ \pm}+X_{i, r_{1} \pm 1}^{ \pm} X_{i, r_{2}}^{ \pm} X_{j, s}^{ \pm}\right)\right. \\
\left.+q^{-1}\left(X_{j, s}^{ \pm} X_{i, r_{1}}^{ \pm} X_{i, r_{2} \pm 1}^{ \pm}-[2]_{q^{2}} X_{i, r_{1}}^{ \pm} X_{j, s}^{ \pm} X_{i, r_{2} \pm 1}^{ \pm}+X_{i, r_{1}}^{ \pm} X_{i, r_{2} \pm 1}^{ \pm} X_{j, s}^{ \pm}\right)\right)=0 \\
\left(i, j \in I_{0}, a_{i j}=-2, k=2, \quad\left(r_{1}, r_{2}\right) \in \mathbb{Z}^{2}, s \in \mathbb{Z}\right),
\end{array}
$$




$$
\begin{aligned}
& \sum_{\sigma \in \mathcal{S}_{2}} \sigma \cdot\left(q ^ { 2 } \left(X_{j, s}^{ \pm} X_{i, r_{1} \pm 2}^{ \pm} X_{i, r_{2}}^{ \pm}-[2]_{q^{3}} X_{i, r_{1} \pm 2}^{ \pm} X_{j, s}^{ \pm} X_{i, r_{2}}^{ \pm}\right.\right. \\
& \left.\quad+X_{i, r_{1} \pm 2}^{ \pm} X_{i, r_{2}}^{ \pm} X_{j, s}^{ \pm}\right)+\left(X_{j, s}^{ \pm} X_{i, r_{1} \pm 1}^{ \pm} X_{i, r_{2} \pm 1}^{ \pm}-[2]_{q^{3}} X_{i, r_{1} \pm 1}^{ \pm} X_{j, s}^{ \pm} X_{i, r_{2} \pm 1}^{ \pm}\right. \\
& \left.\quad+X_{i, r_{1} \pm 1}^{ \pm} X_{i, r_{2} \pm 1}^{ \pm} X_{j, s}^{ \pm}\right)+q^{-2}\left(X_{j, s}^{ \pm} X_{i, r_{1}}^{ \pm} X_{i, r_{2} \pm 2}^{ \pm}\right. \\
& \left.\left.\quad-[2]_{q^{3}} X_{i, r_{1}}^{ \pm} X_{j, s}^{ \pm} X_{i, r_{2} \pm 2}^{ \pm}+X_{i, r_{1}}^{ \pm} X_{i, r_{2} \pm 2}^{ \pm} X_{j, s}^{ \pm}\right)\right)=0 \\
& \quad\left(i, j \in I_{0}, a_{i j}=-3, k=3,\left(r_{1}, r_{2}\right) \in \mathbb{Z}^{2}, s \in \mathbb{Z}\right),
\end{aligned}
$$

where $\varepsilon \in\{ \pm 1\}$ and $\tilde{H}_{i, r}^{ \pm}$and $b_{i j r}$ are defined as follows:

$$
\begin{gathered}
\sum_{r \in \mathbb{Z}} \tilde{H}_{i, \pm r}^{ \pm} u^{r}=\exp \left( \pm\left(q_{i}-q_{i}^{-1}\right) \sum_{r>0} H_{i, \pm r} u^{r}\right) ; \\
b_{i j r}= \begin{cases}0 & \text { if } \tilde{d}_{i, j} \nmid r, \\
{[2 r]_{q}\left(q^{2 r}+(-1)^{r-1}+q^{-2 r}\right) . r} & \text { if }\left(X_{\tilde{n}}^{(k)}, i, j\right)=\left(A_{2 n}^{(2)}, 1,1\right), \\
{\left[\tilde{r} a_{i j}\right]_{q_{i}} / \tilde{r}} & \text { otherwise, with } \tilde{r}=r / \tilde{d}_{i, j} .\end{cases}
\end{gathered}
$$

An isomorphism is given by

$$
C^{ \pm 1} \mapsto \mathcal{C}^{ \pm 1}, \quad k_{i}^{ \pm 1} \mapsto \mathcal{K}_{\tilde{i}}^{ \pm 1}, \quad X_{i, r}^{ \pm} \mapsto \mathcal{X}_{\tilde{i}, r}^{ \pm}, \quad H_{i, s} \mapsto \mathcal{H}_{\tilde{i}, s}
$$

where $(i, r),(i, s) \in I_{0} \times \mathbb{Z}(s \neq 0)$ and ${ }^{\sim}: I_{0} \rightarrow \tilde{I}$ is a section as in Remark 4.10; its inverse is

$$
\mathcal{C}^{ \pm 1} \mapsto C^{ \pm 1}, \quad \mathcal{K}_{i^{\prime}}^{ \pm 1} \mapsto k_{\bar{i}^{\prime}}^{ \pm 1}, \quad \mathcal{X}_{\chi^{u}(\tilde{i}), r}^{ \pm} \mapsto \omega^{u r} X_{i, r}^{ \pm}, \quad \mathcal{H}_{\chi^{u}(\tilde{i}), s} \mapsto \omega^{u s} H_{i, s}
$$

$\left(i^{\prime} \in \tilde{I}, i \in I_{0}, u, r \in \mathbb{Z}, s \in \mathbb{Z} \backslash\{0\}\right)$.

Proof. The claim follows from Remarks 4.4, Corollary 4.6, Remarks 4.11-4.13, Proposition 4.15 and Remarks 4.18-4.24.

Remark 4.26. $\mathcal{U}_{q}^{\mathrm{Dr}}\left(X_{\tilde{n}}^{(k)}\right)$ is (isomorphic to) the $\mathbb{C}(q)$-algebra generated by

$$
C^{ \pm 1}, \quad k_{i}^{ \pm 1}\left(i \in I_{0}\right), \quad X_{i, r}^{ \pm}\left((i, r) \in I_{\mathbb{Z}}\right), \quad H_{i, r}\left((i, r) \in I_{\mathbb{Z}} \backslash\left(I_{0} \times\{0\}\right)\right),
$$

with relations $\left(C U K^{\prime}\right)-\left(S 3^{\prime \pm}\right)$, where, for a relation $(R)$, the relation $\left(R^{\prime}\right)$ is the set of relations in $(R)$ whose left hand side does not involve indices in $\left(I_{0} \times \mathbb{Z}\right) \backslash I_{\mathbb{Z}}$.

Note that the only case where the right hand side of some relation in $\left(R^{\prime}\right)$ involves indices in $\left(I_{0} \times \mathbb{Z}\right) \backslash I_{\mathbb{Z}}$ is $(R)=\left(H X^{ \pm}\right)$: in this situation if $(j, r+s) \notin I_{\mathbb{Z}}$ then $\tilde{d}_{j} \nmid r$ and $b_{i j r}=0$, hence $\left(H X^{\prime \pm}\right)$ is the following relation:

$$
\left[H_{i, r}, X_{j, s}^{ \pm}\right]=\left\{\begin{array}{ll}
0 & \text { if } \tilde{d}_{j} \nmid r \\
\pm b_{i j r} C^{\frac{r F|r|}{2}} X_{j, r+s}^{ \pm} & \text {if } \tilde{d}_{j} \mid r
\end{array} \quad\left((i, r) \in I_{\mathbb{Z}} \backslash\left(I_{0} \times\{0\}\right),(j, s) \in I_{\mathbb{Z}}\right) .\right.
$$


Remark 4.27. Since in the $\mathbb{C}(q)$-algebra generated by $(G)$ for any of the relations $(R)$ defining $\mathcal{U}_{q}^{\operatorname{Dr}}\left(X_{\tilde{n}}^{(k)}\right)$ the relations $(Z X, Z H, R)$ are equivalent to $\left(Z X, Z H, R^{\prime}\right)$, by abuse of notation we shall denote $\left(R^{\prime}\right)$ also by $(R)$.

It is with the presentation of $\mathcal{U}_{q}^{\mathrm{Dr}}$ given in Proposition 4.25 that we shall deal from now on.

\section{§5. More about the definition of $\mathcal{U}_{q}^{\mathrm{Dr}}$}

The material of this section is presented in order to simplify the exposition and to handle more easily the relations defining $\mathcal{U}_{q}^{\text {Dr }}$, with the aim of sharply reducing them: some notation will be fixed; a new formulation will be given, mainly in terms of $q$-commutators, of some of the relations of Proposition 4.25; and some new relations $\left(\left(T 2^{ \pm}\right)\right.$and $\left.\left(T 3^{ \pm}\right)\right)$will be introduced and proved to be equivalent, under suitable conditions, to $\left(S 2^{ \pm}\right)$and $\left(S 3^{ \pm}\right)$. Also the Serre relations are introduced here, but they will be studied in detail in $\S 11$.

Notation 5.1. Let $\mathcal{U}$ be an algebra and let $(R)$ denote the relations

$$
S_{\zeta}(r, s)=0 \quad\left(\zeta \in \mathcal{Z}, r \in \mathbb{Z}^{l}, s \in \mathbb{Z}^{\tilde{l}}\right),
$$

where $\mathcal{Z}$ is a set, $l \in \mathbb{Z}_{+}, \tilde{l} \in\{0,1\}, S_{\zeta}(r, s) \in \mathcal{U}$. Then:

(i) for all $\zeta \in \mathcal{Z}$, denote by $\left(R_{\zeta}\right)$ the relations

$$
S_{\zeta}(r, s)=0 \quad\left(r \in \mathbb{Z}^{l}, s \in \mathbb{Z}^{\tilde{l}}\right)
$$

of course if $\# \mathcal{Z}=1$ and $\mathcal{Z}=\{\zeta\}$ then $(R)=\left(R_{\zeta}\right)$;

(ii) denote by $\mathcal{I}(R)$ the ideal of $\mathcal{U}$ generated by the $S_{\zeta}(r, s)$ 's:

$$
\mathcal{I}(R)=\left(S_{\zeta}(r, s) \mid \zeta \in \mathcal{Z}, r \in \mathbb{Z}^{l}, s \in \mathbb{Z}^{\tilde{l}}\right) ;
$$

of course $\mathcal{I}(R)=\left(\mathcal{I}\left(R_{\zeta}\right) \mid \zeta \in \mathcal{Z}\right)$;

(iii) if $\left({ }^{(h)} R\right)(h=1, \ldots, m)$ are the relations

$$
{ }^{(h)} S_{\zeta}(r, s)=0 \quad\left(\zeta \in{ }^{(h)} \mathcal{Z}, r \in \mathbb{Z}^{l_{h}}, s \in \mathbb{Z}^{\tilde{l}_{h}}\right),
$$

where ${ }^{(h)} \mathcal{Z}$ is a set, $l_{h} \in \mathbb{Z}_{+}, \tilde{l}_{h} \in\{0,1\},{ }^{(h)} S_{\zeta}^{ \pm}(r, s) \in \mathcal{U}$, define

$$
\mathcal{I}\left({ }^{(1)} R, \ldots,{ }^{(m)} R\right)=\left(\mathcal{I}\left({ }^{(1)} R\right), \ldots, \mathcal{I}\left({ }^{(m)} R\right)\right) ;
$$

(iv) if $\left(R^{ \pm}\right)$denotes the relations

$\left(R^{ \pm}\right)$

$$
S_{\zeta}^{ \pm}(r, s)=0 \quad\left(\zeta \in \mathcal{Z}, r \in \mathbb{Z}^{l}, s \in \mathbb{Z}^{\tilde{l}}\right),
$$


where $\mathcal{Z}$ is a set, $l \in \mathbb{Z}_{+}, \tilde{l} \in\{0,1\}, S_{\zeta}^{ \pm}(r, s) \in \mathcal{U}$, denote by $(R)$ the relations

$$
S_{\zeta^{\prime}}(r, s)=0 \quad\left(\zeta^{\prime} \in \mathcal{Z} \times\{ \pm\}, r \in \mathbb{Z}^{l}, s \in \mathbb{Z}^{\tilde{l}}\right),
$$

where $S_{(\zeta, \pm)}(r, s)=S_{\zeta}^{ \pm}(r, s)$; in particular

$$
\mathcal{I}(R)=\left(\mathcal{I}\left(R^{+}\right), \mathcal{I}\left(R^{-}\right)\right)
$$

moreover denote by $\mathcal{I}^{ \pm}(R)$ the ideals

$$
\mathcal{I}^{+}(R)=\mathcal{I}\left(R^{+}\right) \quad \text { and } \quad \mathcal{I}^{-}(R)=\mathcal{I}\left(R^{-}\right) .
$$

Notation 5.2. For $i, j \in I_{0}, l \in \mathbb{N}, a \in \mathbb{Z}, r=\left(r_{1}, \ldots, r_{l}\right) \in \mathbb{Z}^{l}, s \in \mathbb{Z}$ we set

$$
X_{i, j ; l ; a}^{ \pm}(r ; s)=\sum_{u=0}^{l}(-1)^{u}\left[\begin{array}{l}
l \\
u
\end{array}\right]_{q_{i}^{a}} X_{i, r_{1}}^{ \pm} \cdot \ldots \cdot X_{i, r_{u}}^{ \pm} X_{j, s}^{ \pm} X_{i, r_{u+1}}^{ \pm} \cdot \ldots \cdot X_{i, r_{l}}^{ \pm} .
$$

Remark 5.3. The relations $\left(S U L^{ \pm}\right),\left(S 2^{ \pm}\right)$and $\left(S 3^{ \pm}\right)$can be written in a more compact form as:

$$
\sum_{\sigma \in \mathcal{S}_{1-a_{i j}}} \sigma \cdot X_{i, j ; 1-a_{i j} ; 1}^{ \pm}(r ; s)=0
$$

which is $\left(S U L^{ \pm}\right)$, and

$$
\sum_{\sigma \in \mathcal{S}_{2}} \sigma . \sum_{\substack{u, v \geq 0 \\ u+v=-1-a_{i j}}} q^{v-u} X_{i, j ; 2 ;-a_{i j}}^{ \pm}\left(r_{1} \pm v, r_{2} \pm u ; s\right)=0
$$

which is $\left(S 2^{ \pm}\right),\left(S 3^{ \pm}\right)$and also $\left(S U L^{ \pm}\right)$in the case $a_{i j}=-1$.

In order to express the relations $\left(S U L^{ \pm}\right)$in terms of $q$-commutators, and for further use and simplifications, we introduce the following notation.

Notation 5.4. For $i \neq j \in I_{0}, l \in \mathbb{N}, a \in \mathbb{Z}, r=\left(r_{1}, \ldots, r_{l}\right) \in \mathbb{Z}^{l}, s \in \mathbb{Z}$ set

$$
M_{i, j ; l ; a}^{ \pm}\left(r_{1}, \ldots, r_{l} ; s\right)= \begin{cases}X_{j, s}^{ \pm} & \text {if } l=0 \\ {\left[M_{i, j ; l-1 ; a}^{ \pm}\left(r_{1}, \ldots, r_{l-1} ; s\right), X_{i, r_{l}}^{ \pm}\right]_{q_{i}} a_{i j-2 a(l-1)}} & \text { if } l>0 .\end{cases}
$$

Remark 5.5. The relations $\left(S U L^{ \pm}\right)$can be formulated in terms of $q$-commutators as

$$
\sum_{\sigma \in \mathcal{S}_{1-a_{i j}}} \sigma \cdot M_{i, j ; 1-a_{i j} ; 1}^{ \pm}(r ; s)=0
$$

$\left(i \neq j \in I_{0}, a_{i j} \in\{0,-1\}\right.$ if $\left.k \neq 1, r \in \mathbb{Z}^{1-a_{i j}}, s \in \mathbb{Z}\right)$.

Remark 5.6. Also the relations $\left(S 2^{ \pm}\right)$and $\left(S 3^{ \pm}\right)$can be formulated in terms of q-commutators: 
(i) $\left(S 2^{ \pm}\right)$can be written as

$$
\sum_{\sigma \in \mathcal{S}_{2}} \sigma \cdot\left(\left(q^{2}+q^{-2}\right)\left[\left[X_{j, s}^{ \pm}, X_{i, r_{1} \pm 1}^{ \pm}\right]_{q^{2}}, X_{i, r_{2}}^{ \pm}\right]+q^{2}\left[\left[X_{i, r_{1} \pm 1}^{ \pm}, X_{i, r_{2}}^{ \pm}\right]_{q^{2}}, X_{j, s}^{ \pm}\right]_{q^{-4}}\right)=0 ;
$$

(ii) moreover $\left(S 2^{+}\right)$can be written in one of the following equivalent ways:

$$
\begin{gathered}
\sum_{\sigma \in \mathcal{S}_{2}} \sigma \cdot\left(\left(q^{2}+q^{-2}\right)\left[\left[X_{j, s}^{+}, X_{i, r_{1}}^{+}\right]_{q^{-2}}, X_{i, r_{2}+1}^{+}\right]+\left[X_{j, s}^{+},\left[X_{i, r_{2}+1}^{+}, X_{i, r_{1}}^{+}\right]_{q^{2}}\right]_{q^{-4}}\right)=0 \\
\sum_{\sigma \in \mathcal{S}_{2}} \sigma \cdot\left(\left[\left[X_{j, s}^{+}, X_{i, r_{1}+1}^{+}\right]_{q^{-2}}, X_{i, r_{2}}^{+}\right]-q^{2}\left[X_{i, r_{1}+1}^{+},\left[X_{j, s}^{+}, X_{i, r_{2}}^{+}\right]_{q^{-2}}\right]_{q^{-4}}\right)=0
\end{gathered}
$$

(iii) $\left(S 3^{ \pm}\right)$can be formulated in terms of $q$-commutators as follows:

$$
\begin{aligned}
\sum_{\sigma \in \mathcal{S}_{2}} \sigma \cdot\left(\left(q^{2}+q^{-4}\right)[\right. & {\left.\left[X_{j, s}^{ \pm}, X_{i, r_{1} \pm 2}^{ \pm}\right]_{q^{3}}, X_{i, r_{2}}^{ \pm}\right]_{q^{-1}} } \\
& +\left(1-q^{-2}+q^{-4}\right)\left[\left[X_{j, s}^{ \pm}, X_{i, r_{1} \pm 1}^{ \pm}\right]_{q^{3}}, X_{i, r_{2} \pm 1}^{ \pm}\right]_{q} \\
& \left.+q^{2}\left[\left[X_{i, r_{1} \pm 2}^{ \pm}, X_{i, r_{2}}^{ \pm}\right]_{q^{2}}+\left[X_{i, r_{2} \pm 1}^{ \pm}, X_{i, r_{1} \pm 1}^{ \pm}\right]_{q^{2}}, X_{j, s}^{ \pm}\right]_{q^{-6}}\right)=0 .
\end{aligned}
$$

Definition 5.7. Consider the case $k>1, X_{\tilde{n}}^{(k)} \neq A_{2 n}^{(2)}$ and introduce the relations $\left(T k^{ \pm}\right)$:

$$
\begin{array}{r}
\left(T 2^{ \pm}\right) \quad \sum_{\sigma \in \mathcal{S}_{2}} \sigma \cdot\left[\left[X_{j, s}^{ \pm}, X_{i, r_{1} \pm 1}^{ \pm}\right]_{q^{2}}, X_{i, r_{2}}^{ \pm}\right]=0 \quad\left(i, j \in I_{0}, a_{i j}=-2, r \in \mathbb{Z}^{2}, s \in \mathbb{Z}\right) ; \\
\left(T 3^{ \pm}\right) \quad \sum_{\sigma \in \mathcal{S}_{2}} \sigma \cdot\left(\left(q^{2}+1\right)\left[\left[X_{j, s}^{ \pm}, X_{i, r_{1} \pm 2}^{ \pm}\right]_{q^{3}}, X_{i, r_{2}}^{ \pm}\right]_{q^{-1}}+\left[\left[X_{j, s}^{ \pm}, X_{i, r_{1} \pm 1}^{ \pm}\right]_{q^{3}}, X_{i, r_{2} \pm 1}^{ \pm}\right]_{q}\right)=0 \\
\left(i, j \in I_{0}, a_{i j}=-3, r \in \mathbb{Z}^{2}, s \in \mathbb{Z}\right) .
\end{array}
$$

Proposition 5.8. Let $k>1, X_{\tilde{n}}^{(k)} \neq A_{2 n}^{(2)}$. Then $\mathcal{I}\left(X 1^{ \pm}, S k^{ \pm}\right)=\mathcal{I}\left(X 1^{ \pm}, T k^{ \pm}\right)$. More precisely if $i, j \in I_{0}$ are such that $a_{i j}<-1$ we have

$$
\left.\mathcal{I}\left(X 1_{i}^{ \pm}, S k^{ \pm}\right)=\mathcal{I}\left(X 1_{i}^{ \pm}, T k^{ \pm}\right) \quad \text { (see Notation } 5.1(\mathrm{i})\right) .
$$

In particular, $\left(S 2^{ \pm}\right)$and $\left(S 3^{ \pm}\right)$can be replaced respectively by $\left(T 2^{ \pm}\right)$and $\left(T 3^{ \pm}\right)$ among the defining relations of $\mathcal{U}_{q}^{\mathrm{Dr}}$.

Proof. It is enough to notice that

$$
\left[\sum_{\sigma \in \mathcal{S}_{2}} \sigma \cdot\left[X_{i, r_{1} \pm 1}^{ \pm}, X_{i, r_{2}}^{ \pm}\right]_{q^{2}}, X_{j, s}^{ \pm}\right]_{q^{-4}}
$$

and

$$
\left[\left[X_{i, r_{1} \pm 2}^{ \pm}, X_{i, r_{2}}^{ \pm}\right]_{q^{2}}+\left[X_{i, r_{2} \pm 1}^{ \pm}, X_{i, r_{1} \pm 1}^{ \pm}\right]_{q^{2}}, X_{j, s}^{ \pm}\right]_{q^{-6}}
$$

belong to $\mathcal{I}\left(X 1_{i}^{ \pm}\right)$. 
Definition 5.9. We also recall the Serre relations

$$
\sum_{\sigma \in \mathcal{S}_{1-a_{i j}}} \sigma \cdot X_{i, j ; 1-a_{i j} ; 1}^{ \pm}(r ; s)=0 \quad\left(i \neq j \in I_{0}, r \in \mathbb{Z}^{1-a_{i j}}, s \in \mathbb{Z}\right) .
$$

Remark 5.10. The Serre relations can be formulated in terms of $q$-commutators as

$$
\sum_{\sigma \in \mathcal{S}_{1-a_{i j}}} \sigma \cdot M_{i, j ; 1-a_{i j} ; 1}^{ \pm}(r ; s)=0 \quad\left(i \neq j \in I_{0}, r \in \mathbb{Z}^{1-a_{i j}}, s \in \mathbb{Z}\right) .
$$

Remark 5.11. The right hand sides of relations $\left(T k^{ \pm}\right)$and $\left(S^{ \pm}\right)$are zero, hence Remark 4.27 holds for these relations (see also Remark 4.26).

The comparison of the defining relations of $\mathcal{U}_{q}^{\mathrm{Dr}}$ with the Serre relations is the subject of $\S 11$.

Notation 5.12. Let us also introduce the following notation:

(i) for $i, j \in I_{0}$ and $r, s \in \mathbb{Z}$,

$$
M_{(2)}^{ \pm}((i, r),(j, s))=\left[X_{i, r \pm \tilde{d}_{i j}}^{ \pm}, X_{j, s}^{ \pm}\right]_{q_{i}^{a_{i j}}}+\left[X_{j, s \pm \tilde{d}_{i j}}^{ \pm}, X_{i, r}^{ \pm}\right]_{q_{j}{ }^{a_{j i}}} ;
$$

(ii) for $i \in I_{0}$ and $r=\left(r_{1}, r_{2}\right) \in \mathbb{Z}^{2}$,

$$
M_{i}^{ \pm}(r)=\left[X_{i, r_{1} \pm \tilde{d}_{i}}^{ \pm}, X_{i, r_{2}}^{ \pm}\right]_{q_{i}^{2}} ;
$$

(iii) if $X_{\tilde{n}}^{(k)}=A_{2 n}^{(2)}$ and $r=\left(r_{1}, r_{2}\right) \in \mathbb{Z}^{2}$,

$$
M_{(2,2)}^{ \pm}(r)=\left[X_{1, r_{1} \pm 2}^{ \pm}, X_{1, r_{2}}^{ \pm}\right]_{q^{2}}-q^{4}\left[X_{1, r_{1} \pm 1}^{ \pm}, X_{1, r_{2} \pm 1}^{ \pm}\right]_{q^{-6}} ;
$$

(iv) if $X_{\tilde{n}}^{(k)}=A_{2 n}^{(2)}$ and $r=\left(r_{1}, r_{2}, r_{3}\right) \in \mathbb{Z}^{3}$,

$$
M_{(3)}^{\varepsilon, \pm}(r)=\left[\left[X_{1, r_{1} \pm \varepsilon}^{ \pm}, X_{1, r_{2}}^{ \pm}\right]_{q^{2 \varepsilon}}, X_{1, r_{3}}^{ \pm}\right]_{q^{4 \varepsilon}} ;
$$

(v) if $k>1$ and $r=\left(r_{1}, r_{2}\right) \in \mathbb{Z}^{2}, s \in \mathbb{Z}$,

$$
X_{[k]}^{ \pm}(r ; s)=\sum_{\substack{u, v \geq 0 \\ u+v=k-1}} q^{v-u} X_{i, j ; 2 ; k}^{ \pm}\left(r_{1} \pm v, r_{2} \pm u ; s\right)
$$

where $i, j \in I_{0}$ are such that $a_{i j}=-k$;

(vi) if $k=2, X_{\tilde{n}}^{(k)} \neq A_{2 n}^{(2)}$ and $r=\left(r_{1}, r_{2}\right) \in \mathbb{Z}^{2}, s \in \mathbb{Z}$,

$$
M_{[2]}^{ \pm}(r ; s)=M_{i, j ; 2 ; 1}^{ \pm}\left(r_{1} \pm 1, r_{2} ; s\right)
$$

where $i, j \in I_{0}$ are such that $a_{i j}=-2$; 
(vii) if $k=3$ and $r=\left(r_{1}, r_{2}\right) \in \mathbb{Z}^{2}, s \in \mathbb{Z}$,

$$
M_{[3]}^{ \pm}(r ; s)=\left(q^{2}+1\right) M_{i, j ; 2 ; 2}^{ \pm}\left(r_{1} \pm 2, r_{2} ; s\right)+M_{i, j ; 2 ; 1}^{ \pm}\left(r_{1} \pm 1, r_{2} \pm 1 ; s\right)
$$

where $i, j \in I_{0}$ are such that $a_{i j}=-3$.

Remark 5.13. Of course the following relations depend on $\left(Z X^{ \pm}\right)$:

(i) $M_{(2)}^{ \pm}((i, r),(j, s))=0$ if $(r, s) \notin \tilde{d}_{i} \mathbb{Z} \times \tilde{d}_{j} \mathbb{Z}$;

(ii) $M_{i}^{ \pm}(r)=0$ if $r \notin\left(\tilde{d}_{i} \mathbb{Z}\right)^{2}$;

(iii) $M_{i, j ; l ; a}^{ \pm}(r ; s)=0$ if $(r, s) \notin\left(\tilde{d}_{i} \mathbb{Z}\right)^{l} \times \tilde{d}_{j} \mathbb{Z}$;

(iv) $X_{i, j ; l ; a}^{ \pm}(r ; s)=0$ if $(r, s) \notin\left(\tilde{d}_{i} \mathbb{Z}\right)^{l} \times \tilde{d}_{j} \mathbb{Z}$;

(v) $X_{[k]}^{ \pm}(r ; s)=0$ if $s \notin \tilde{d} \mathbb{Z}$;

(vi) $M_{[k]}^{ \pm}(r ; s)=0$ if $s \notin k \mathbb{Z}$.

Remark 5.14. Recalling Remark 4.27 (and Remark 5.13) we have the following obvious reformulation of the relations $\left(X D^{ \pm}\right)-\left(S 3^{ \pm}\right),\left(T 2^{ \pm}\right),\left(T 3^{ \pm}\right)$and $\left(S^{ \pm}\right)$in terms of the notation just introduced $(5.2,5.4,5.12)$ :

$$
\begin{array}{lcl}
\begin{array}{l}
\left(X D^{ \pm}\right) \\
\left(X 1^{ \pm}\right)
\end{array} & M_{(2)}^{ \pm}\left(\left(i, \tilde{d}_{i} r\right),\left(j, \tilde{d}_{j} s\right)\right)=0 \quad\left(i, j \in I_{0}, a_{i j}<0, r, s \in \mathbb{Z}\right) ; \\
\left(X 2^{ \pm}\right) & \sum_{\sigma \in \mathcal{S}_{2}} \sigma \cdot M_{i}^{ \pm}\left(\tilde{d}_{i} r\right)=0 \quad\left(i \in I_{0}, \quad\left(X_{\tilde{n}}^{(k)}, i\right) \neq\left(A_{2 n}^{(2)}, i\right), r \in \mathbb{Z}^{2}\right) ; \\
\left(X 3^{\varepsilon, \pm}\right) & \sum_{\sigma \in \mathcal{S}_{2}} \sigma \cdot M_{(2,2)}^{ \pm}(r)=0 \quad\left(r \in \mathbb{Z}^{2}\right) ; \\
\left(S(U L)^{ \pm}\right) & \sum_{\sigma \in \mathcal{S}_{3}} \sigma \cdot M_{(3)}^{\varepsilon, \pm}(r)=0 \quad\left(r \in \mathbb{Z}^{3}\right) ; \\
\sum_{\sigma \in \mathcal{S}_{1-a_{i j}}} \sigma \cdot M_{i, j ; 1-a_{i j} ; 1}^{ \pm}\left(\tilde{d}_{i} r ; \tilde{d}_{j} s\right)=0
\end{array}
$$

or equivalently

$$
\sum_{\sigma \in \mathcal{S}_{1-a_{i j}}} \sigma \cdot X_{i, j ; 1-a_{i j} ; 1}^{ \pm}\left(\tilde{d}_{i} r ; \tilde{d}_{j} s\right)=0
$$

$\left(i \neq j \in I_{0}\left(a_{i j} \in\{0,-1\}\right.\right.$ if $\left.\left.k \neq 1\right), r \in \mathbb{Z}^{1-a_{i j}}, s \in \mathbb{Z}\right)$

$$
\begin{array}{ll}
\sum_{\sigma \in \mathcal{S}_{2}} \sigma \cdot X_{[k]}^{ \pm}(r ; \tilde{d} s)=0 & \left(r \in \mathbb{Z}^{2}, s \in \mathbb{Z}\right) \\
\sum_{\sigma \in \mathcal{S}_{2}} \sigma \cdot M_{[k]}^{ \pm 1}(r ; k s)=0 & \left(r \in \mathbb{Z}^{2}, s \in \mathbb{Z}\right) .
\end{array}
$$




\section{§6. $\overline{\mathcal{U}}_{q}^{\mathrm{Dr}}$ and its structures}

In order to study the relations defining $\mathcal{U}_{q}^{\mathrm{Dr}}$ it is convenient to proceed by steps: the algebras $\overline{\mathcal{U}}_{q}^{\mathrm{Dr}}=\overline{\mathcal{U}}_{q}^{\mathrm{Dr}}\left(X_{\tilde{n}}^{(k)}\right)$ and $\tilde{\mathcal{U}}_{q}^{\mathrm{Dr}}=\tilde{\mathcal{U}}_{q}^{\mathrm{Dr}}\left(X_{\tilde{n}}^{(k)}\right)$ here defined are such that $\tilde{\mathcal{U}}_{q}^{\text {Dr }}$ is a quotient of $\overline{\mathcal{U}}_{q}^{\text {Dr }}$ and $\mathcal{U}_{q}^{\text {Dr }}$ is a quotient of $\tilde{\mathcal{U}}_{q}^{\text {Dr }}$.

This section is devoted to introduce some important structures on $\overline{\mathcal{U}}_{q}^{\mathrm{Dr}}(Q$ gradation, homomorphisms between some of these algebras, automorphisms and antihomomorphisms of each of them), which will be proved to induce analogous structures on $\tilde{\mathcal{U}}_{q}^{\text {Dr }}$ (see also $\S 7$ ) and, what is finally important, on $\mathcal{U}_{q}^{\text {Dr }}$ (see also $\S 7$ and $\S 8)$.

Some remarks point out the first (trivially) unnecessary relations: $(Z H)$ and $(K H)$ are redundant.

Definition 6.1. We denote by:

(i) $\tilde{\mathcal{U}}_{q}^{\operatorname{Dr}}\left(X_{\tilde{n}}^{(k)}\right)$ the $\mathbb{C}(q)$-algebra generated by $(G)$ with the relations $\left(Z X^{ \pm}\right)$, $(C U K),(C K),\left(K X^{ \pm}\right),(X X)$ and

$\left(H X L^{ \pm}\right) \quad\left[H_{i, r}, X_{j, s}^{ \pm}\right]= \pm b_{i j r} C^{\frac{r \mp|r|}{2}} X_{j, r+s}^{ \pm} \quad\left((i, r),(j, s) \in I_{\mathbb{Z}}, \tilde{d}_{i} \leq|r| \leq \tilde{d}_{i j}\right) ;$

(ii) $\overline{\mathcal{U}}_{q}^{\mathrm{Dr}}\left(X_{\tilde{n}}^{(k)}\right)$ the $\mathbb{C}(q)$-algebra generated by

$$
C^{ \pm 1}, \quad k_{i}^{ \pm 1}\left(i \in I_{0}\right), \quad X_{i, r}^{ \pm}\left((i, r) \in I_{0} \times \mathbb{Z}\right)
$$

with the relations $\left(Z X^{ \pm}\right),(C U K),(C K)$.

Remark 6.2. $\mathcal{U}_{q}^{\mathrm{Dr}}\left(X_{\tilde{n}}^{(k)}\right)$ is obviously a quotient of $\tilde{\mathcal{U}}_{q}^{\mathrm{Dr}}\left(X_{\tilde{n}}^{(k)}\right)$.

We shall prove that also $\tilde{\mathcal{U}}_{q}^{\mathrm{Dr}}\left(X_{\tilde{n}}^{(k)}\right)$ is a quotient of $\overline{\mathcal{U}}_{q}^{\mathrm{Dr}}\left(X_{\tilde{n}}^{(k)}\right)$.

Since $\overline{\mathcal{U}}_{q}^{\operatorname{Dr}}\left(X_{\tilde{n}}^{(k)}\right) \rightarrow \tilde{\mathcal{U}}_{q}^{\operatorname{Dr}}\left(X_{\tilde{n}}^{(k)}\right)$ is obviously well defined, we just need to prove that this map is surjective, or equivalently that $\tilde{\mathcal{U}}_{q}^{\operatorname{Dr}}\left(X_{\tilde{n}}^{(k)}\right)$ is generated by $(\bar{G})$. To this end we need some simple remarks.

Remark 6.3. In $\tilde{\mathcal{U}}_{q}^{\mathrm{Dr}}$ (hence in $\mathcal{U}_{q}^{\mathrm{Dr}}$ ) the following hold:

(i) $\tilde{H}_{i, 0}^{ \pm}=1$ for all $i \in I_{0}$;

(ii) $\tilde{H}_{i, \mp r}^{ \pm}=0$ for all $i \in I_{0}$ and $r>0$;

(iii) for each $r>0, \tilde{H}_{i, \pm r}^{ \pm} \mp\left(q_{i}-q_{i}^{-1}\right) H_{i, \pm r}$ belongs to the $\mathbb{C}(q)$-subalgebra generated by $\left\{H_{i, \pm s} \mid 0<s<r\right\}$; in particular $\left\{H_{i, \pm s} \mid(i, s) \in I_{\mathbb{Z}}, 0<s<r\right\}$ and $\left\{\tilde{H}_{i, \pm s}^{ \pm} \mid(i, s) \in I_{\mathbb{Z}}, 0<s<r\right\}$ generate the same $\mathbb{C}(q)$-subalgebra.

Remark 6.4. In $\tilde{\mathcal{U}}_{q}^{\mathrm{Dr}}$ (hence in $\mathcal{U}_{q}^{\mathrm{Dr}}$ ), for all $i \in I_{0}$ and $r \in \mathbb{Z}_{+}$we have

$$
\tilde{H}_{i, \pm r}^{ \pm}=\left(q_{i}-q_{i}^{-1}\right) k_{i}^{\mp 1}\left[X_{i, \pm r}^{ \pm}, X_{i, 0}^{\mp}\right] \text {. }
$$


In particular for all $(i, r) \in I_{0} \times \mathbb{Z}, \tilde{H}_{i, r}^{ \pm}$lies in the subalgebra of $\tilde{\mathcal{U}}_{q}^{\operatorname{Dr}}\left(X_{\tilde{n}}^{(k)}\right)$ generated by $(\bar{G})$.

Consequently (see Remark 6.3), for all $(i, r) \in I_{0} \times \mathbb{Z}$ also $H_{i, r}$ lies in the subalgebra of $\tilde{\mathcal{U}}_{q}^{\mathrm{Dr}}\left(X_{\tilde{n}}^{(k)}\right)$ generated by $(\bar{G})$.

Corollary 6.5. (i) $\tilde{\mathcal{U}}_{q}^{\operatorname{Dr}}\left(X_{\tilde{n}}^{(k)}\right)$ and $\mathcal{U}_{q}^{\operatorname{Dr}}\left(X_{\tilde{n}}^{(k)}\right)$ are generated by $(\bar{G})$;

(ii) $\tilde{\mathcal{U}}_{q}^{\mathrm{Dr}}\left(X_{\tilde{n}}^{(k)}\right)$ is a quotient of $\overline{\mathcal{U}}_{q}^{\operatorname{Dr}}\left(X_{\tilde{n}}^{(k)}\right)$.

Notation 6.6. We denote by $\tilde{H}_{i, \pm r}^{ \pm}$also the elements in $\overline{\mathcal{U}}_{q}^{\mathrm{Dr}}$ defined by

$$
\tilde{H}_{i, \pm r}^{ \pm}= \begin{cases}\left(q_{i}-q_{i}^{-1}\right) k_{i}^{-1}\left[X_{i, r}^{+}, X_{i, 0}^{-}\right] & \text {if } r, \pm r>0, \\ \left(q_{i}-q_{i}^{-1}\right)\left[X_{i,-r}^{-}, X_{i, 0}^{+}\right] k_{i} & \text { if } r>0, \pm r<0, \\ 1 & \text { if } r=0, \\ 0 & \text { if } r<0,\end{cases}
$$

and by $H_{i, r}$ the elements of $\overline{\mathcal{U}}_{q}^{\mathrm{Dr}}$ defined by

$$
\sum_{r \in \mathbb{Z}} \tilde{H}_{i, \pm r}^{ \pm} u^{r}=\exp \left( \pm\left(q_{i}-q_{i}^{-1}\right) \sum_{r>0} H_{i, \pm r} u^{r}\right) .
$$

Remark 6.7. The relations $(Z H)$ are trivial in $\overline{\mathcal{U}}_{q}^{\operatorname{Dr}}\left(X_{\tilde{n}}^{(k)}\right)$.

Remark 6.8. (i) $\overline{\mathcal{U}}_{q}^{\mathrm{Dr}}=\overline{\mathcal{U}}_{q}^{\mathrm{Dr}}\left(X_{\tilde{n}}^{(k)}\right)$ is $Q$-graded:

$$
\overline{\mathcal{U}}_{q}^{\mathrm{Dr}}=\bigoplus_{\alpha \in Q} \overline{\mathcal{U}}_{q, \alpha}^{\mathrm{Dr}},
$$

where $C^{ \pm 1}, k_{i}^{ \pm 1} \in \overline{\mathcal{U}}_{q, 0}^{\mathrm{Dr}}, X_{i, r}^{ \pm} \in \overline{\mathcal{U}}_{q, \pm \alpha_{i}+r \delta}^{\mathrm{Dr}}$ for all $i \in I_{0}$ and $r \in \mathbb{Z}$, and $\overline{\mathcal{U}}_{q, \alpha}^{\mathrm{Dr}} \overline{\mathcal{U}}_{q, \beta}^{\mathrm{Dr}} \subseteq \overline{\mathcal{U}}_{q, \alpha+\beta}^{\mathrm{Dr}}$.

(ii) $\tilde{H}_{i, r}^{ \pm}(r \in \mathbb{Z})$ and $H_{i, r}(r \in \mathbb{Z} \backslash\{0\})$ are homogeneous of degree $r \delta$ for all $i \in I_{0}$.

(iii) Since the relations defining $\tilde{\mathcal{U}}_{q}^{\mathrm{Dr}}$ and $\mathcal{U}_{q}^{\mathrm{Dr}}$ are homogeneous, the $Q$-gradation of $\overline{\mathcal{U}}_{q}^{\text {Dr }}$ induces $Q$-gradations on $\tilde{\mathcal{U}}_{q}^{\mathrm{Dr}}=\bigoplus_{\alpha \in Q} \tilde{\mathcal{U}}_{q, \alpha}^{\mathrm{Dr}}$ and on $\mathcal{U}_{q}^{\mathrm{Dr}}=\bigoplus_{\alpha \in Q} \mathcal{U}_{q, \alpha}^{\mathrm{Dr}}$.

Notation 6.9. The $\mathbb{C}(q)$-algebra $\mathbb{C}(q)\left[C^{ \pm 1}, k_{i}^{ \pm 1} \mid i \in I_{0}\right]$ is $Q$-graded, with onedimensional homogeneous components $\mathbb{C}(q) k_{\alpha}(\alpha \in Q)$ where we set

$$
k_{m \delta+\sum_{i \in I_{0}} m_{i} \alpha_{i}}=C^{m} \prod_{i \in I_{0}} k_{i}^{m_{i}} \quad\left(m, m_{i} \in \mathbb{Z} \forall i \in I_{0}\right) .
$$

Indeed $\mathbb{C}(q)\left[C^{ \pm 1}, k_{i}^{ \pm 1} \mid i \in I_{0}\right]=\mathbb{C}(q)[Q]$.

Recall that $\mathbb{C}(q)\left[C^{ \pm 1}, k_{i}^{ \pm 1} \mid i \in I_{0}\right]$ naturally maps into $\overline{\mathcal{U}}_{q, 0}^{\mathrm{Dr}} \subseteq \overline{\mathcal{U}}_{q}^{\mathrm{Dr}}$ (hence into $\tilde{\mathcal{U}}_{q, 0}^{\mathrm{Dr}} \subseteq \tilde{\mathcal{U}}_{q}^{\mathrm{Dr}}$ and into $\left.\mathcal{U}_{q, 0}^{\mathrm{Dr}} \subseteq \mathcal{U}_{q}^{\mathrm{Dr}}\right)$. 
Remark 6.10. (i) The relations $(C U K),(C K)$ and $\left(K X^{ \pm}\right)$are equivalent to (a) and (b) where:

(a) the $\mathbb{C}(q)$-subalgebra generated by $\left\{C^{ \pm 1}, k_{i}^{ \pm 1} \mid i \in I_{0}\right\}$ is a quotient of the ring of Laurent polynomials $\mathbb{C}(q)\left[k_{i}^{ \pm 1} \mid i \in I\right]\left(C=\prod_{i \in I} k_{i}^{r_{i}}\right)$;

(b) for all $\alpha, \beta \in Q$ and all $x$ of degree $\beta$ we have $k_{\alpha} x=q^{(\alpha \mid \beta)} x k_{\alpha}$.

(ii) The relations $(K H)$ depend on $(C U K),(C K)$ and $\left(K X^{ \pm}\right)$, and in particular are trivial in $\tilde{\mathcal{U}}_{q}^{\operatorname{Dr}}\left(X_{\tilde{n}}^{(k)}\right)$.

Definition 6.11. We denote by $\mathcal{F}_{q}^{+}=\mathcal{F}_{q}^{+}\left(X_{\tilde{n}}^{(k)}\right)$ and $\mathcal{F}_{q}^{-}=\mathcal{F}_{q}^{-}\left(X_{\tilde{n}}^{(k)}\right)$ the $\mathbb{C}(q)$ algebras generated respectively by

$$
X_{i, r}^{+} \quad\left((i, r) \in I_{0} \times \mathbb{Z}\right)
$$

and

$\left(G^{-}\right)$

$$
X_{i, r}^{-} \quad\left((i, r) \in I_{0} \times \mathbb{Z}\right)
$$

with relations respectively $\left(Z X^{+}\right)$and $\left(Z X^{-}\right)$.

Remark 6.12. $\mathcal{F}_{q}^{+}\left(X_{\tilde{n}}^{(k)}\right)$ and $\mathcal{F}_{q}^{-}\left(X_{\tilde{n}}^{(k)}\right)$ are the free $\mathbb{C}(q)$-algebras generated respectively by

$$
\left(G^{+}\right) \quad X_{i, r}^{+} \quad\left((i, r) \in I_{\mathbb{Z}}\right)
$$

and

$\left(G^{-}\right)$

$$
X_{i, r}^{-} \quad\left((i, r) \in I_{\mathbb{Z}}\right) .
$$

Notation 6.13. $\mathcal{F}_{q}^{+}$and $\mathcal{F}_{q}^{-}$naturally embed in $\overline{\mathcal{U}}_{q}^{\text {Dr }}$, hence they map in $\tilde{\mathcal{U}}_{q}^{\text {Dr }}$ and in $\mathcal{U}_{q}^{\mathrm{Dr}}$; their images in $\tilde{\mathcal{U}}_{q}^{\mathrm{Dr}}$ are denoted respectively by $\tilde{\mathcal{U}}_{q}^{\mathrm{Dr},+}$ and $\tilde{\mathcal{U}}_{q}^{\mathrm{Dr},-}$, and their images in $\mathcal{U}_{q}^{\mathrm{Dr}}$ by $\mathcal{U}_{q}^{\mathrm{Dr},+}$ and $\mathcal{U}_{q}^{\mathrm{Dr},-}$.

Remark 6.14. (i) As subalgebras of $\overline{\mathcal{U}}_{q}^{\mathrm{Dr}}, \mathcal{F}_{q}^{+}$inherits a $\left(Q_{0,+} \oplus \mathbb{Z} \delta\right)$-gradation and $\mathcal{F}_{q}^{-}$inherits a $\left(-Q_{0,+} \oplus \mathbb{Z} \delta\right)$-gradation;

(ii) more precisely,

$$
\mathcal{F}_{q}^{ \pm} \subseteq \mathbb{C}(q) \oplus \bigoplus_{\substack{\alpha \in Q_{0,+}, \alpha \neq 0 \\ m \in \mathbb{Z}}} \overline{\mathcal{U}}_{q, \pm \alpha+m \delta}^{\mathrm{Dr}}
$$

and similarly

$$
\begin{aligned}
& \tilde{\mathcal{U}}_{q}^{\mathrm{Dr}, \pm} \subseteq \mathbb{C}(q) \oplus \bigoplus_{\substack{\alpha \in Q_{0,+, \alpha \neq 0} \\
m \in \mathbb{Z}}} \tilde{\mathcal{U}}_{q, \pm \alpha+m \delta}^{\mathrm{Dr}}, \\
& \mathcal{U}_{q}^{\mathrm{Dr}, \pm} \subseteq \mathbb{C}(q) \oplus \bigoplus_{\substack{\alpha \in Q_{0,+, \alpha \neq 0} \\
m \in \mathbb{Z}}} \mathcal{U}_{q, \pm \alpha+m \delta}^{\mathrm{Dr}} .
\end{aligned}
$$


The last part of this section is devoted to the definition of automorphisms and antiautomorphisms of the algebras just introduced, which make evident some symmetries in the generators and relations of $\mathcal{U}_{q}^{\mathrm{Dr}}$. Thanks to these structures the study of the apparently very complicated relations defining $\mathcal{U}_{q}^{\mathrm{Dr}}$ will be strongly simplified in $\S 7, \S 9$ and the following sections.

The next definitions depend on the choice of an automorphism $\eta$ of $\mathbb{C}$. A short discussion about the choice of $\eta$ is in Remark 6.17.

Definition 6.15. Let us introduce the following homomorphisms and antihomomorphisms:

(i) $\bar{\Omega}: \overline{\mathcal{U}}_{q}^{\mathrm{Dr}} \rightarrow \overline{\mathcal{U}}_{q}^{\mathrm{Dr}}$ is the antihomomorphism defined on the generators by

$$
\left.\bar{\Omega}\right|_{\mathbb{C}}=\eta, \quad q \mapsto q^{-1}, \quad C^{ \pm 1} \mapsto C^{\mp 1}, \quad k_{i}^{ \pm 1} \mapsto k_{i}^{\mp 1}, \quad X_{i, r}^{ \pm} \mapsto X_{i,-r}^{\mp} .
$$

(ii) $\Theta_{\mathcal{F}}^{+}: \mathcal{F}_{q}^{+} \rightarrow \mathcal{F}_{q}^{+}$and $\Theta_{\mathcal{F}}^{-}: \mathcal{F}_{q}^{-} \rightarrow \mathcal{F}_{q}^{-}$are the homomorphisms defined on the generators by

$$
\begin{array}{llll}
\Theta_{\mathcal{F}}^{+}: & \left.\Theta_{\mathcal{F}}^{+}\right|_{\mathbb{C}}=\eta, & q \mapsto q^{-1}, & X_{i, r}^{+} \mapsto X_{i,-r}^{+}, \\
\Theta_{\mathcal{F}}^{-}: & \left.\Theta_{\mathcal{F}}^{-}\right|_{\mathbb{C}}=\eta, & q \mapsto q^{-1}, & X_{i, r}^{-} \mapsto X_{i,-r}^{-} .
\end{array}
$$

(iii) $\bar{\Theta}: \overline{\mathcal{U}}_{q}^{\mathrm{Dr}} \rightarrow \overline{\mathcal{U}}_{q}^{\mathrm{Dr}}$ is the homomorphism defined on the generators by

$$
\begin{gathered}
\left.\bar{\Theta}\right|_{\mathbb{C}}=\eta, \quad q \mapsto q^{-1}, \quad C^{ \pm 1} \mapsto C^{ \pm 1}, \quad k_{i}^{ \pm 1} \mapsto k_{i}^{\mp 1}, \\
X_{i, r}^{+} \mapsto-X_{i,-r}^{+} k_{i} C^{-r}, \quad X_{i, r}^{-} \mapsto-k_{i}^{-1} C^{-r} X_{i,-r}^{-} .
\end{gathered}
$$

(iv) For all $i \in I_{0}, \bar{t}_{i}: \overline{\mathcal{U}}_{q}^{\mathrm{Dr}} \rightarrow \overline{\mathcal{U}}_{q}^{\mathrm{Dr}}$ is the $\mathbb{C}(q)$-homomorphism defined on the generators by

$$
C^{ \pm 1} \mapsto C^{ \pm 1}, \quad k_{j}^{ \pm 1} \mapsto\left(k_{j} C^{-\delta_{i j} \tilde{d}_{i}}\right)^{ \pm 1}, \quad X_{j, r}^{ \pm} \mapsto X_{j, r \mp \delta_{i j} \tilde{d}_{i}}^{ \pm}
$$

(v) For $i \in I_{0}$ let

$$
\bar{\phi}_{i}: \begin{cases}\overline{\mathcal{U}}_{q}^{\operatorname{Dr}}\left(A_{1}^{(1)}\right) \rightarrow \overline{\mathcal{U}}_{q}^{\operatorname{Dr}}\left(X_{\tilde{n}}^{(k)}\right) & \text { if }\left(X_{\tilde{n}}^{(k)}, i\right) \neq\left(A_{2 n}^{(2)}, 1\right) \\ \overline{\mathcal{U}}_{q}^{\operatorname{Dr}}\left(A_{2}^{(2)}\right) \rightarrow \overline{\mathcal{U}}_{q}^{\operatorname{Dr}}\left(X_{\tilde{n}}^{(k)}\right) & \text { if }\left(X_{\tilde{n}}^{(k)}, i\right)=\left(A_{2 n}^{(2)}, 1\right)\end{cases}
$$

be the $\mathbb{C}$-homomorphisms defined on the generators as follows:

$$
q \mapsto q_{i}, \quad C^{ \pm 1} \mapsto C^{ \pm \tilde{d}_{i}}, \quad k^{ \pm 1} \mapsto k_{i}^{ \pm 1}, \quad X_{r}^{ \pm} \mapsto X_{i, \tilde{d}_{i} r}^{ \pm} .
$$

Remark 6.16. It is immediate to notice that:

(i) $\bar{\Omega}, \Theta_{\mathcal{F}}^{ \pm}, \bar{\Theta}, \bar{t}_{i}$ and $\bar{\phi}_{i}$ are all well-defined;

(ii) $\bar{\Omega}\left(\mathcal{F}_{q}^{ \pm}\right)=\mathcal{F}_{q}^{\mp}$;

(iii) $\bar{\Omega}$ and $\bar{\Theta}$ are involutions of $\overline{\mathcal{U}}_{q}^{\mathrm{Dr}}$ if $\eta$ is an involution of $\mathbb{C}$; 
(iv) the $\bar{t}_{i}$ 's are automorphisms of $\overline{\mathcal{U}}_{q}^{\text {Dr }}$ (of infinite order) for all $i \in I_{0}$; more precisely $\left\langle\bar{t}_{i} \mid i \in I_{0}\right\rangle \cong \mathbb{Z}^{I_{0}}$

(v) the following commutation properties hold:

$$
\bar{\Theta} \bar{\Omega}=\bar{\Omega} \bar{\Theta}, \quad \bar{t}_{i} \bar{\Omega}=\bar{\Omega}_{i}, \quad \bar{t}_{i} \bar{\Theta}=\bar{\Theta}_{i}^{-1}, \quad \bar{t}_{i} \bar{t}_{j}=\bar{t}_{j} \bar{t}_{i} \quad \forall i, j \in I_{0}
$$

as maps of $\overline{\mathcal{U}}_{q}^{\mathrm{Dr}}\left(X_{\tilde{n}}^{(k)}\right)$ into itself; moreover, for all $i \in I_{0}$,

$$
\bar{\Omega} \bar{\phi}_{i}=\bar{\phi}_{i} \bar{\Omega}, \quad \bar{\Theta} \bar{\phi}_{i}=\bar{\phi}_{i} \bar{\Theta}, \quad \bar{t}_{i} \bar{\phi}_{i}=\bar{\phi}_{i} \bar{t}_{1}, \quad \bar{t}_{j} \bar{\phi}_{i}=\bar{\phi}_{i} \quad \forall j \in I_{0} \backslash\{i\}
$$

as maps from $\overline{\mathcal{U}}_{q}^{\mathrm{Dr}}\left(A_{1}^{(1)}\right)$ to $\overline{\mathcal{U}}_{q}^{\mathrm{Dr}}\left(X_{\tilde{n}}^{(k)}\right)$ if $\left(X_{\tilde{n}}^{(k)}, i\right) \neq\left(A_{2 n}^{(2)}, 1\right)$, and from $\overline{\mathcal{U}}_{q}^{\mathrm{Dr}}\left(A_{2}^{(2)}\right)$ to $\overline{\mathcal{U}}_{q}^{\operatorname{Dr}}\left(X_{\tilde{n}}^{(k)}\right)$ if $\left(X_{\tilde{n}}^{(k)}, i\right)=\left(A_{2 n}^{(2)}, 1\right) ;$

(vi) for all $\alpha=\beta+m \delta \in Q$ with $\beta \in Q_{0}, m \in \mathbb{Z}$ we have

$$
\begin{aligned}
& \bar{\Omega}\left(k_{\alpha}\right)=k_{-\alpha}, \quad \bar{\Theta}\left(k_{\beta+m \delta}\right)=k_{-\beta+m \delta}, \quad \bar{t}_{i}\left(k_{\alpha}\right)=k_{\lambda_{i}(\alpha)}, \\
& \bar{\Omega}\left(\overline{\mathcal{U}}_{q, \alpha}^{\mathrm{Dr}}\right)=\overline{\mathcal{U}}_{q,-\alpha}^{\mathrm{Dr}}, \quad \bar{\Theta}\left(\overline{\mathcal{U}}_{q, \beta+m \delta}^{\mathrm{Dr}}\right)=\overline{\mathcal{U}}_{q, \beta-m \delta}^{\mathrm{Dr}}, \quad \bar{t}_{i}\left(\overline{\mathcal{U}}_{q, \alpha}^{\mathrm{Dr}}\right)=\overline{\mathcal{U}}_{q, \lambda_{i}(\alpha)}^{\mathrm{Dr}} ;
\end{aligned}
$$

moreover for all $m_{1}, m \in \mathbb{Z}$ and $i \in I_{0}$,

$\bar{\phi}_{i}\left(k_{m_{1} \alpha_{1}+m \delta}\right)=k_{m_{1} \alpha_{i}+\tilde{d}_{i} m \delta} \quad$ and $\quad \bar{\phi}_{i}\left(\mathcal{U}_{q, m_{1} \alpha_{1}+m \delta}^{\mathrm{Dr}}\left(A_{*}^{(*)}\right)\right) \subseteq \mathcal{U}_{q, m_{1} \alpha_{i}+\tilde{d}_{i} m \delta}^{\mathrm{Dr}}\left(X_{\tilde{n}}^{(k)}\right)$;

(vii) on the elements $H_{i, r}$ and $\tilde{H}_{i, r}^{ \pm}$we have

$$
\begin{gathered}
\bar{\Omega}\left(\tilde{H}_{i, r}^{ \pm}\right)=\tilde{H}_{i,-r}^{\mp}, \quad \bar{\Omega}\left(H_{i, r}\right)=H_{i,-r} \\
\bar{\phi}_{i}\left(\tilde{H}_{1, r}^{ \pm}\right)=\tilde{H}_{i, \tilde{d}_{i} r}^{ \pm}, \quad \bar{\phi}_{i}\left(H_{1, r}\right)=H_{i, \tilde{d}_{i} r} \quad \forall i \in I_{0} .
\end{gathered}
$$

Remark 6.17. For the purpose of the present paper, the definition of $\bar{\Omega}, \Theta_{\mathcal{F}}^{ \pm}, \bar{\Theta}$ given in Definition 6.15 could be simplified by requiring these maps to be $\mathbb{C}$ linear (that is, $\eta=\mathrm{id}_{\mathbb{C}}$ ). But the choice of a nontrivial automorphism $\eta$ of $\mathbb{C}$ becomes sometimes necessary, as when specializing $q$ at a complex value $\epsilon \neq \pm 1$ : indeed a homomorphism defined over $\mathbb{C}(q)$ (and mapping $q$ to $q^{-1}$ ) induces a homomorphism on the specialization at $\epsilon$ if and only if the ideal $(q-\epsilon)$ is stable; if, for example, $\epsilon$ is a root of 1 , this could be obtained by choosing $\eta(z)=\bar{z}$ for all $z \in \mathbb{C}$, that is, by requiring the homomorphism to be $\mathbb{C}$-antilinear. For this reason, from now on we suppose $\eta$ to be the conjugation on $\mathbb{C}$, that is $\bar{\Omega}, \Theta_{\mathcal{F}}^{ \pm}, \bar{\Theta}$ to be $\mathbb{C}$-antilinear (see Definitions 8.2 and 8.4, and compare also with Definition 3.3).

Of course one needs to pay more attention and eventually choose a different $\eta$ when one is interested in specializing at complex values $\epsilon$ such that $|\epsilon| \neq 1$.

Our goal is of course to show that $\bar{\Omega}, \bar{\Theta}, \bar{t}_{i}$ and $\bar{\phi}_{i}$ induce $\Omega, \Theta, t_{i}$ and $\phi_{i}$ on $\mathcal{U}_{q}^{\mathrm{Dr}}$. This is indeed very easy to show, but we take this opportunity to simplify the relations that we have to deal with, passing through $\tilde{\mathcal{U}}_{q}^{\text {Dr }}$ for two reasons: 
underlining the first redundancies of the relations (see Corollary 7.17); and discussing separately the relations $\left(X D^{+}\right)-\left(S 3^{+}\right)$whose first simplification can be made simultaneously, as examples of a general case (see $\S 9$ ).

\section{$\S 7$. The algebra $\tilde{\mathcal{U}}_{q}^{\mathrm{Dr}}$}

The algebra $\tilde{\mathcal{U}}_{q}^{\mathrm{Dr}}$ and its structures, to which this section is devoted, play a fundamental role in the study and simplification of the relations $\left(X D^{ \pm}\right)-\left(S 3^{ \pm}\right)$. In particular the relations are analyzed by underlining their consequences on the (anti)automorphisms $\tilde{\Omega}$, $\tilde{\Theta}$ and $\tilde{t}_{i}\left(i \in I_{0}\right)$; the relations $\left(H X^{ \pm}\right)$and $(H H)$ are proved to be redundant; and much smaller sets of generators are provided.

Remark 7.1. Remarks 6.10(i) and 6.16(vi) imply immediately that $\bar{\Omega}, \bar{\Theta}, \overline{t_{i}}$ and $\bar{\phi}_{i}$ preserve the relations $\left(K X^{ \pm}\right)$.

Remark 7.2. For all $i \in I_{0}, \bar{\phi}_{i}$ obviously induces

$$
\tilde{\phi}_{i}: \begin{cases}\tilde{\mathcal{U}}_{q}^{\operatorname{Dr}}\left(A_{1}^{(1)}\right) \rightarrow \tilde{\mathcal{U}}_{q}^{\operatorname{Dr}}\left(X_{\tilde{n}}^{(k)}\right) & \text { if }\left(X_{\tilde{n}}^{(k)}, i\right) \neq\left(A_{2 n}^{(2)}, 1\right), \\ \tilde{\mathcal{U}}_{q}^{\operatorname{Dr}}\left(A_{2}^{(2)}\right) \rightarrow \tilde{\mathcal{U}}_{q}^{\operatorname{Dr}}\left(X_{\tilde{n}}^{(k)}\right) & \text { if }\left(X_{\tilde{n}}^{(k)}, i\right)=\left(A_{2 n}^{(2)}, 1\right),\end{cases}
$$

and

$$
\phi_{i}: \begin{cases}\mathcal{U}_{q}^{\operatorname{Dr}}\left(A_{1}^{(1)}\right) \rightarrow \mathcal{U}_{q}^{\operatorname{Dr}}\left(X_{\tilde{n}}^{(k)}\right) & \text { if }\left(X_{\tilde{n}}^{(k)}, i\right) \neq\left(A_{2 n}^{(2)}, 1\right), \\ \mathcal{U}_{q}^{\operatorname{Dr}}\left(A_{2}^{(2)}\right) \rightarrow \mathcal{U}_{q}^{\operatorname{Dr}}\left(X_{\tilde{n}}^{(k)}\right) & \text { if }\left(X_{\tilde{n}}^{(k)}, i\right)=\left(A_{2 n}^{(2)}, 1\right) .\end{cases}
$$

Remark 7.3. (i) $\bar{\Omega}\left(\mathcal{I}^{+}(H X L)\right)=\mathcal{I}^{-}(H X L)$ and $\bar{\Omega}\left(\mathcal{I}^{+}(H X)\right)=\mathcal{I}^{-}(H X)$;

(ii) $\bar{\Omega}$ preserves the relations $\left(H X L^{ \pm}\right)$and $\left(H X^{ \pm}\right)$.

Notation 7.4. Define relations $(X X D),(X X E),\left(X X H^{+}\right)$and $\left(X X H^{-}\right)$by:

$$
\begin{array}{rlrl}
(X X D) & {\left[X_{i, r}^{+}, X_{j, s}^{-}\right]} & =0 \quad\left((i, r),(j, s) \in I_{\mathbb{Z}}, i \neq j\right), \\
(X X E) & {\left[X_{i, r}^{+}, X_{i,-r}^{-}\right]=\frac{C^{r} k_{i}-C^{-r} k_{i}^{-1}}{q_{i}-q_{i}^{-1}} \quad\left((i, r) \in I_{\mathbb{Z}}\right),} \\
\left(X X H^{+}\right) & {\left[X_{i, r}^{+}, X_{i, s}^{-}\right]=\frac{C^{-s} k_{i} \tilde{H}_{i, r+s}^{+}}{q_{i}-q_{i}^{-1}} \quad\left((i, r),(i, s) \in I_{\mathbb{Z}}, r+s>0\right),} \\
\left(X X H^{-}\right) & {\left[X_{i, r}^{+}, X_{i, s}^{-}\right]=-\frac{C^{-r} \tilde{H}_{i, r+s}^{-} k_{i}^{-1}}{q_{i}-q_{i}^{-1}} \quad\left((i, r),(i, s) \in I_{\mathbb{Z}}, r+s<0\right),}
\end{array}
$$

Remark 7.5. (i) $\mathcal{I}(X X)=\mathcal{I}(X X D, X X E, X X H)$;

(ii) $\bar{\Omega}(\mathcal{I}(X X D))=\mathcal{I}(X X D)$ and $\bar{\Omega}(\mathcal{I}(X X E))=\mathcal{I}(X X E)$;

(iii) $\bar{\Omega}\left(\mathcal{I}^{+}(X X H)\right)=\mathcal{I}^{-}(X X H)$

(iv) $\bar{\Omega}$ preserves the relations $(X X)$.

Corollary 7.6. $\bar{\Omega}$ induces $\tilde{\Omega}: \tilde{\mathcal{U}}_{q}^{\mathrm{Dr}} \rightarrow \tilde{\mathcal{U}}_{q}^{\mathrm{Dr}}$. 
Remark 7.7. (i) $\bar{t}_{i}(\mathcal{I}(X X D))=\mathcal{I}(X X D)$ and $\bar{t}_{i}(\mathcal{I}(X X E))=\mathcal{I}(X X E)$ for all $i \in I_{0}$;

(ii) $\mathcal{I}(X X D, X X E)$ is the $\bar{t}_{i}^{ \pm 1}$-stable ideal (for all $i \in I_{0}$ ) generated by

$$
\left\{\left[X_{i, 0}^{+}, X_{j, 0}^{-}\right]-\delta_{i j} \frac{k_{i}-k_{i}^{-1}}{q_{i}-q_{i}^{-1}} \mid i, j \in I_{0}\right\} .
$$

We want to show now that for all $i \in I_{0}, \bar{t}_{i}$ induces $\tilde{t}_{i}: \tilde{\mathcal{U}}_{q}^{\mathrm{Dr}} \rightarrow \tilde{\mathcal{U}}_{q}^{\mathrm{Dr}}$. Since $\bar{t}_{i}$ commutes with $\bar{\Omega}$, Remarks $7.1,7.3(\mathrm{i}), 7.5(\mathrm{i}) \&($ iii) and $7.7(\mathrm{i})$ imply that it is enough to concentrate on $\mathcal{I}^{+}(H X L), \mathcal{I}^{+}(X X H)$.

Remark 7.8. (i) Note that if $r+s>0$,

$$
\left(q_{i}-q_{i}^{-1}\right) C^{s} k_{i}^{-1}\left[X_{i, r}^{+}, X_{i, s}^{-}\right]=\bar{t}_{i}^{s / \tilde{d}_{i}}\left(\left(q_{i}-q_{i}^{-1}\right) k_{i}^{-1}\left[X_{i, r+s}^{+}, X_{i, 0}^{-}\right]\right)=\bar{t}_{i}^{s / \tilde{d}_{i}}\left(\tilde{H}_{i, r+s}^{+}\right),
$$

so that the relations $\left(X X H^{+}\right)$are equivalent to

$$
\bar{t}_{i}^{s}\left(\tilde{H}_{i, r}^{+}\right)=\tilde{H}_{i, r}^{+} \quad \forall i \in I_{0}, r>0, s \in \mathbb{Z} ;
$$

(ii) $\bar{t}_{i}^{ \pm 1}\left(\mathcal{I}^{+}(X X H)\right)=\mathcal{I}^{+}(X X H)$ for all $i \in I_{0}$;

(iii) $\mathcal{I}^{+}(X X H)$ is the $\bar{t}_{i}^{ \pm 1}$-stable ideal (for all $\left.i \in I_{0}\right)$ generated by

$$
\left\{\bar{t}_{i}\left(\tilde{H}_{i, r}^{+}\right)-\tilde{H}_{i, r}^{+} \mid i \in I_{0}, r>0\right\} .
$$

Remark 7.9. Note that for all $(i, r) \in I_{\mathbb{Z}} \backslash\left(I_{0} \times\{0\}\right),(j, s) \in I_{\mathbb{Z}}$ and $h \in I_{0}$,

$$
\begin{aligned}
\bar{t}_{h}^{ \pm 1}\left(\left[H_{i, r}, X_{j, s}^{+}\right]-b_{i j r} C^{\frac{r-|r|}{2}} X_{j, r+s}^{+}\right) \\
=\left[\bar{t}_{h}^{ \pm 1}\left(H_{i, r}\right), X_{j, s \mp \delta_{j h} \tilde{d}_{j}}^{+}\right]-b_{i j r} C^{\frac{r-|r|}{2}} X_{j, r+s \mp \delta_{j h} \tilde{d}_{j}}^{+}
\end{aligned}
$$

Then, thanks to Remark 7.8 and to the definition of $\bar{t}_{i}$ (see Definition 6.15), we have:

(i) $\bar{t}_{i}^{ \pm 1}\left(\mathcal{I}^{+}(H X)\right) \subseteq \mathcal{I}^{+}(X X H, H X)$;

(ii) $\bar{t}_{i}^{ \pm 1}\left(\mathcal{I}^{+}(H X L)\right) \subseteq \mathcal{I}^{+}(X X H, H X L)$;

(iii) $\mathcal{I}^{+}(X X H, H X L)$ is the $\bar{t}_{i}^{ \pm 1}$-stable ideal (for all $\left.i \in I_{0}\right)$ generated by

$$
\left\{\bar{t}_{i}\left(\tilde{H}_{i, r}^{+}\right)-\tilde{H}_{i, r}^{+},\left[H_{i, s}, X_{j, 0}^{+}\right]-b_{i j s} C^{\frac{s-|s|}{2}} X_{j, s}^{+}\left|i \in I_{0}, r>0, \tilde{d}_{i} \leq\right| s \mid \leq \tilde{d}_{i j}\right\} .
$$

Corollary 7.10. (i) For all $i \in I_{0}, \bar{t}_{i}$ induces $\tilde{t}_{i}: \tilde{\mathcal{U}}_{q}^{\mathrm{Dr}} \rightarrow \tilde{\mathcal{U}}_{q}^{\mathrm{Dr}}$;

(ii) for all $i, j \in I_{0}, \tilde{t}_{i}\left(\tilde{H}_{j, r}^{+}\right)=\tilde{H}_{j, r}^{+}$for each $r \in \mathbb{Z}$ and $\tilde{t}_{i}\left(H_{j, r}\right)=H_{j, r}$ for each $r \in \mathbb{Z} \backslash\{0\}$.

We come now to $\bar{\Theta}$ recalling that $\bar{\Theta} \bar{\Omega}=\bar{\Omega} \bar{\Theta}$ and $\bar{\Theta} \bar{t}_{i}^{ \pm 1}=\bar{t}_{i}^{\mp 1} \bar{\Theta}$ for all $i \in I_{0}$. 
Remark 7.11. Notice that $\left[X_{i, 0}^{+}, X_{j, 0}^{-}\right]-\delta_{i j} \frac{k_{i}-k_{i}^{-1}}{q_{i}-q_{i}^{-1}}$ is fixed by $\bar{\Theta}$; hence, thanks to Remark 7.7(ii), $\mathcal{I}(X X D, X X E)$ is $\bar{\Theta}$-stable.

Remark 7.12. (i) For all $i \in I_{0}$ and $r>0$,

$$
\bar{\Theta}\left(\tilde{H}_{i, r}^{+}\right)=\bar{t}_{i}^{r / \tilde{d}_{i}}\left(\tilde{H}_{i,-r}^{-}\right)+\left(q_{i}-q_{i}^{-1}\right)\left[X_{i,-r}^{+} X_{i, 0}^{-}, k_{i}\right] C^{-r}
$$

(ii) for all $i \in I_{0}$ and $r>0, \bar{\Theta}\left(\tilde{H}_{i, \pm r}^{ \pm}\right)-\tilde{H}_{i, \mp r}^{\mp}$ and $\bar{\Theta}\left(H_{i, \pm r}\right)-H_{i, \mp r}$ lie in $\mathcal{I}\left(K X, X X H^{\mp}\right)$;

(iii) for all $i \in I_{0}$ and $r>0$,

$$
\bar{\Theta}\left(\bar{t}_{i}\left(\tilde{H}_{i, r}^{+}\right)-\tilde{H}_{i, r}^{+}\right)=\bar{t}_{i}^{-1}\left(\bar{\Theta}\left(\tilde{H}_{i, r}^{+}\right)\right)-\bar{\Theta}\left(\tilde{H}_{i, r}^{+}\right) \in \mathcal{I}\left(K X, X X H^{-}\right) ;
$$

(iv) for all $i, j \in I_{0}, \tilde{d}_{i} \leq|r| \leq \tilde{d}_{i j}, s \in \mathbb{Z}$,

$$
\begin{aligned}
\bar{\Theta}\left(\left[H_{i, r},\right.\right. & \left.\left.X_{j, s}^{+}\right]-b_{i j r} C^{\frac{r-|r|}{2}} X_{j, r+s}^{+}\right) \\
& =-\left[\bar{\Theta}\left(H_{i, r}\right), X_{j,-s}^{+} k_{j} C^{-s}\right]+b_{i j r} C^{\frac{r-|r|}{2}} X_{j,-(r+s)}^{+} k_{j} C^{-(r+s)} \\
& =-\left(\left[\bar{\Theta}\left(H_{i, r}\right), X_{j,-s}^{+} k_{j}\right] k_{j}^{-1}-b_{i j r} C^{\frac{-r-|r|}{2}} X_{j,-(r+s)}^{+}\right) k_{j} C^{-s}
\end{aligned}
$$

belongs to $\mathcal{I}\left(K X, X X H, H X L^{+}\right)$.

Then:

(v) $\bar{\Theta}\left(\mathcal{I}^{+}(X X H)\right) \subseteq \mathcal{I}\left(K X, X X H^{-}\right)$;

(vi) $\bar{\Theta}\left(\mathcal{I}^{+}(H X L)\right) \subseteq \mathcal{I}\left(K X, X X H, H X L^{+}\right)$;

(vii) $\mathcal{I}(K X, X X H)$ and $\mathcal{I}\left(K X, X X H, H X L^{ \pm}\right)$are $\bar{\Theta}$-stable.

Corollary 7.13. (i) $\bar{\Theta}$ induces $\tilde{\Theta}: \tilde{\mathcal{U}}_{q}^{\mathrm{Dr}} \rightarrow \tilde{\mathcal{U}}_{q}^{\mathrm{Dr}}$;

(ii) for all $i \in I_{0}, \tilde{\Theta}\left(\tilde{H}_{i, r}^{+}\right)=\tilde{H}_{i,-r}^{-}$for each $r \in \mathbb{Z}$ and $\tilde{\Theta}\left(H_{i, r}\right)=H_{i,-r}$ for each $r \in \mathbb{Z} \backslash\{0\}$.

Remark 7.14. (i) Let $f: Q_{0,+} \rightarrow \mathbb{Z}$ be defined by

$$
f(0)=0, \quad f\left(\alpha+\alpha_{i}\right)=f(\alpha)+\left(\alpha \mid \alpha_{i}\right) \quad \forall \alpha \in Q_{0,+}, i \in I_{0} ;
$$

notice that $f$ is well defined, because $\left(\alpha \mid \alpha_{i}\right)+\left(\alpha+\alpha_{i} \mid \alpha_{j}\right)=\left(\alpha \mid \alpha_{j}\right)+$ $\left(\alpha+\alpha_{j} \mid \alpha_{i}\right)$.

(ii) For all $X^{+} \in \mathcal{F}_{q, \alpha+m \delta}^{+}$and $X^{-} \in \mathcal{F}_{q,-\alpha+m \delta}^{-}$(where $\alpha \in Q_{0,+}, m \in \mathbb{Z}$ ) we find that in $\tilde{\mathcal{U}}_{q}^{\mathrm{Dr}}$,

$$
\begin{aligned}
& \tilde{\Theta} \pi^{+}\left(X^{+}\right)=(-1)^{h} q^{f(\alpha)} \pi^{+} \Theta_{\mathcal{F}}^{+}\left(X^{+}\right) k_{\alpha} C^{-m}, \\
& \tilde{\Theta} \pi^{-}\left(X^{-}\right)=(-1)^{h} q^{-f(\alpha)} C^{-m} k_{-\alpha} \pi^{-} \Theta_{\mathcal{F}}^{-}\left(X^{-}\right),
\end{aligned}
$$

where $\pi^{ \pm}: \mathcal{F}_{q}^{ \pm} \rightarrow \tilde{\mathcal{U}}_{q}^{\mathrm{Dr}}$ is the restriction to $\mathcal{F}_{q}^{ \pm}$of the natural projection $\overline{\mathcal{U}}_{q}^{\mathrm{Dr}} \rightarrow \tilde{\mathcal{U}}_{q}^{\mathrm{Dr}}$, and $h=\sum_{i \in I_{0}} m_{i}$ if $\alpha=\sum_{i \in I_{0}} m_{i} \alpha_{i}$. 
(iii) In particular $\tilde{\Theta} \pi^{ \pm}\left(X^{ \pm}\right)$and $\pi^{ \pm} \Theta_{\mathcal{F}}^{ \pm}\left(X^{ \pm}\right)$are equal up to invertible elements of $\tilde{\mathcal{U}}_{q}^{\mathrm{Dr}}$.

We now present some more remarks about generators and relations of $\tilde{\mathcal{U}}_{q}^{\mathrm{Dr}}$. For the next proposition see the analogous results for $\mathcal{U}_{q}^{\mathrm{DJ}}$ in $[\mathrm{Be}]$ and [Da].

Proposition 7.15. In $\tilde{\mathcal{U}}_{q}^{\mathrm{Dr}}$ we have $\mathcal{I}^{ \pm}(H X) \subseteq \mathcal{I}^{ \pm}(X D, X 1, X 2)$.

Proof. In order to avoid repetitive computations we use the behaviour of the relations $\left(X D^{ \pm}\right),\left(X 1^{ \pm}\right)$and $\left(X 2^{ \pm}\right)$under the action of $\tilde{\Omega}$, $\tilde{\Theta}$ and $\tilde{t}_{i}\left(i \in I_{0}\right)$, which is an independent result proved in Remarks 9.8 and 9.20; here it allows us to reduce to the study of $\left[H_{i, r}, X_{j, 0}^{+}\right]$with $r>0$. Indeed:

$$
\begin{gathered}
\tilde{t}_{j}^{s}\left(\left[H_{i, r}, X_{j, 0}^{+}\right]\right)=\left[H_{i, r}, X_{j,-\tilde{d}_{j} s}^{+}\right] \quad \text { and } \quad \tilde{t}_{j}^{s}\left(X_{j, r}^{+}\right)=X_{j, r-\tilde{d}_{j} s}^{+}, \\
{\left[H_{i, r}, X_{j, s}^{+}\right]=0 \quad \text { and } \quad b_{i j r} X_{j, r+s}^{+}=0 \quad \text { if } \tilde{d}_{j} \nmid s,} \\
\tilde{\Theta}\left(\left[H_{i, r}, X_{j, s}^{+}\right]\right)=-\left[H_{i,-r}, X_{j,-s}^{+}\right] k_{j} C^{-s} \text { and } \tilde{\Theta}\left(X_{j, r+s}^{+}\right)=-X_{j,-r-s}^{+} k_{j} C^{-r-s}, \\
\tilde{\Omega}\left(\left[H_{i, r}, X_{j, s}^{+}\right]\right)=-\left[H_{i,-r}, X_{j,-s}^{-}\right] \quad \text { and } \quad \tilde{\Omega}\left(C^{\frac{r-|r|}{2}} X_{j, r+s}^{+}\right)=C^{\frac{-r+|r|}{2}} X_{j,-r-s}^{-},
\end{gathered}
$$

and of course $b_{i j r}=\tilde{t}_{j}\left(b_{i j r}\right)=\tilde{\Theta}\left(b_{i j r}\right)=\tilde{\Omega}\left(b_{i j r}\right)=b_{i j-r}$.

Given an element $x \in \tilde{\mathcal{U}}_{q}^{\text {Dr }}$ define the operators ${ }_{l} x$ and ${ }_{r} x$ on $\tilde{\mathcal{U}}_{q}^{\text {Dr }}$ respectively as left and right multiplication by $x$; if we have elements $x_{s} \in \tilde{\mathcal{U}}_{q}^{\mathrm{Dr}}(s \in \mathbb{N})$ set ${ }_{l} x(u)=\sum_{s \in \mathbb{N}} l x_{s} u^{s}$ and ${ }_{r} x(u)=\sum_{s \in \mathbb{N} r} x_{s} u^{s}$; notice that if $f: \tilde{\mathcal{U}}_{q}^{\mathrm{Dr}} \rightarrow \tilde{\mathcal{U}}_{q}^{\mathrm{Dr}}$ is such that $f\left(x_{s}\right)=x_{s}$ for all $s \in \mathbb{N}$ then ${ }_{l} x(u)$ and ${ }_{r} x(u)$ commute with $f$.

Let $i, j \in I_{0}$ : we want to study $\left({ }_{l} \tilde{H}_{i}^{+}(u)-{ }_{r} \tilde{H}_{i}^{+}(u)\right)\left(X_{j, s}^{+}\right)$and deduce from it $\left({ }_{l} H_{i}(u)-{ }_{r} H_{i}(u)\right)\left(X_{j, s}^{+}\right)$(setting $\left.H_{i, 0}=0\right)$. To this end, note that

$$
{ }_{l} \tilde{H}_{i}^{+}(u)=\exp \left(\left(q_{i}-q_{i}^{-1}\right)_{l} H_{i}(u)\right), \quad{ }_{r} \tilde{H}_{i}^{+}(u)=\exp \left(\left(q_{i}-q_{i}^{-1}\right)_{r} H_{i}(u)\right)
$$

and both commute with $\tilde{t}_{j}$.

The next computations are performed in $\tilde{\mathcal{U}}_{q}^{\text {Dr }} / \mathcal{I}^{+}(X D, X 1, X 2)$.

Remarking that $\left(r>\tilde{d}_{i j}\right)$

$$
\begin{aligned}
{\left[\tilde{H}_{i, r}^{+}, X_{j, 0}^{+}\right] } & =\left(q_{i}-q_{i}^{-1}\right) k_{i}^{-1}\left[\left[X_{i, r}^{+}, X_{i, 0}^{-}\right], X_{j, 0}^{+}\right]_{q_{i} a_{i j}} \\
& =\left(q_{i}-q_{i}^{-1}\right) k_{i}^{-1}\left(\left[X_{i, r}^{+},\left[X_{i, 0}^{-}, X_{j, 0}^{+}\right]\right]_{q_{i} a_{i j}}-\left[X_{i, 0}^{-},\left[X_{i, r}^{+}, X_{j, 0}^{+}\right]_{\left.q_{i} a_{i j}\right]}\right]\right) \\
& =\left(q_{i}-q_{i}^{-1}\right) k_{i}^{-1}\left(q_{i}^{a_{i j}}\left[\delta_{i j} \frac{k_{i}-k_{i}^{-1}}{q_{i}-q_{i}^{-1}}, X_{i, r}^{+}\right]_{q_{i}^{-a_{i j}}}+\left[\left[X_{i, r}^{+}, X_{j, 0}^{+}\right]_{q_{i} a_{i j}}, X_{i, 0}^{-}\right]\right) \\
& =\left(q_{i}-q_{i}^{-1}\right) k_{i}^{-1}\left(\delta_{i j}[2]_{q_{i}} k_{i} X_{i, r}^{+}+\left[\left[X_{i, r}^{+}, X_{j, 0}^{+}\right]_{q_{i} a_{i j}}, X_{i, 0}^{-}\right]\right),
\end{aligned}
$$

let us distinguish two cases: 
(i) $\left(X_{\tilde{n}}^{(k)}, i, j\right) \neq\left(A_{2 n}^{(2)}, 1,1\right)$ : then, thanks to $\left(X D^{+}\right),\left(X 1^{+}\right)$and $\left(H X L^{+}\right)$,

$$
\begin{aligned}
& {\left[\tilde{H}_{i, r}^{+}, X_{j, 0}^{+}\right]=\left(q_{i}-q_{i}^{-1}\right) k_{i}^{-1}\left(\delta_{i j}[2]_{q_{i}} k_{i} X_{i, r}^{+}-\left[\left[X_{j, \tilde{d}_{i j}}^{+}, X_{i, r-\tilde{d}_{i j}}^{+}\right]_{q_{i}}^{a_{i j}}, X_{i, 0}^{-}\right]\right)} \\
& =\left(q_{i}-q_{i}^{-1}\right) k_{i}^{-1}\left(\delta_{i j}[2]_{q_{i}} k_{i} X_{i, r}^{+}-\left[X_{j, \tilde{d}_{i j}}^{+},\left[X_{i, r-\tilde{d}_{i j}}^{+}, X_{i, 0}^{-}\right]\right]_{q_{i}^{a_{i j}}}\right. \\
& \left.+q_{i}^{a_{i j}}\left[X_{i, r-\tilde{d}_{i j}}^{+},\left[X_{j, \tilde{d}_{i j}}^{+}, X_{i, 0}^{-}\right]\right]_{q_{i}^{-a_{i j}}}\right) \\
& =\left(q_{i}-q_{i}^{-1}\right) \delta_{i j}\left([2]_{q_{i}} X_{i, r}^{+}-\frac{1}{q_{i}-q_{i}^{-1}}\left[\tilde{H}_{i, \tilde{d}_{i}}^{+}, X_{i, r-\tilde{d}_{i}}^{+}\right]\right) \\
& +\left(q_{i}-q_{i}^{-1}\right) q_{i}^{a_{i j}} k_{i}^{-1}\left[\left[X_{i, r-\tilde{d}_{i j}}^{+}, X_{i, 0}^{-}\right], X_{j, \tilde{d}_{i j}}^{+}\right]_{q_{i}^{-a_{i j}}} \\
& =\left(q_{i}-q_{i}^{-1}\right) \delta_{i j}\left([2]_{q_{i}} X_{i, r}^{+}-\left[H_{i, \tilde{d}_{i}}, X_{i, r-\tilde{d}_{i}}^{+}\right]\right)+q_{i}^{a_{i j}}\left[\tilde{H}_{i, r-\tilde{d}_{i j}}^{+}, X_{j, \tilde{d}_{i j}}^{+}\right]_{q_{i}}^{-2 a_{i j}} \\
& =q_{i}^{a_{i j}} \tilde{H}_{i, r-\tilde{d}_{i j}}^{+} X_{j, \tilde{d}_{i j}}^{+}-q_{i}^{-a_{i j}} X_{j, \tilde{d}_{i j}}^{+} \tilde{H}_{i, r-\tilde{d}_{i j}}^{+} ;
\end{aligned}
$$

hence, using again $\left(H X L^{+}\right)$,

$$
\left({ }_{l} \tilde{H}_{i}^{+}(u)-{ }_{r} \tilde{H}_{i}^{+}(u)\right)\left(X_{j, 0}^{+}\right)=\left(q_{i}^{a_{i j}}{ }_{l} \tilde{H}_{i}^{+}(u)-q_{i}^{-a_{i j}}{ }_{r} \tilde{H}_{i}^{+}(u)\right) \tilde{t}_{j}^{-\tilde{d}_{i j} / \tilde{d}_{j}} u^{\tilde{d}_{i j}}\left(X_{j, 0}^{+}\right),
$$

or equivalently

$$
{ }_{l} \tilde{H}_{i}^{+}(u)\left(1-q_{i}^{a_{i j}} \tilde{t}_{j}^{-\tilde{d}_{i j} / \tilde{d}_{j}} u^{\tilde{d}_{i j}}\right)\left(X_{j, 0}^{+}\right)={ }_{r} \tilde{H}_{i}^{+}(u)\left(1-q_{i}^{-a_{i j}} \tilde{t}_{j}^{-\tilde{d}_{i j} / \tilde{d}_{j}} u^{\tilde{d}_{i j}}\right)\left(X_{j, 0}^{+}\right) ;
$$

from this we get

$$
\begin{aligned}
\left(q_{i}-q_{i}^{-1}\right) & \left({ }_{l} H_{i}(u)-{ }_{r} H_{i}(u)\right)\left(X_{j, 0}^{+}\right) \\
= & \left(\log \left(1-q_{i}^{-a_{i j}} \tilde{t}_{j}^{-\tilde{d}_{i j} / \tilde{d}_{j}} u^{\tilde{d}_{i j}}\right)-\log \left(1-q_{i}^{a_{i j}} \tilde{t}_{j}^{-\tilde{d}_{i j} / \tilde{d}_{j}} u^{\tilde{d}_{i j}}\right)\right)\left(X_{j, 0}^{+}\right),
\end{aligned}
$$

that is,

$$
\left[H_{i, r}, X_{j, 0}^{+}\right]= \begin{cases}0 & \text { if } \tilde{d}_{i j} \nmid r, \\ \frac{q_{i}^{r a_{i j} / \tilde{d}_{i j}}-q_{i}^{-r a_{i j} / \tilde{d}_{i j}}}{\left(r / \tilde{d}_{i j}\right)\left(q_{i}-q_{i}^{-1}\right)} X_{j, r}^{+}=b_{i j r} X_{j, r}^{+} & \text {otherwise. }\end{cases}
$$

(ii) $\left(X_{\tilde{n}}^{(k)}, i, j\right)=\left(A_{2 n}^{(2)}, 1,1\right)$ : the computations are a little more complicated than in case (i), but substantially similar; we separate the cases $r=2$ and $r>2$ and, thanks to $\left(X 2^{+}\right)$and $\left(H X L^{+}\right)$, we get

$$
\begin{aligned}
{\left[\tilde{H}_{1,2}^{+}\right.} & \left., X_{1,0}^{+}\right]=\left(q-q^{-1}\right) k_{1}^{-1}\left([2]_{q} k_{1} X_{1,2}^{+}+\left[\left[X_{1,2}^{+}, X_{1,0}^{+}\right]_{q^{2}}, X_{1,0}^{-}\right]\right) \\
& =\left(q-q^{-1}\right) k_{1}^{-1}\left([2]_{q} k_{1} X_{1,2}^{+}+\left(q^{4}-q^{-2}\right)\left[\left(X_{1,1}^{+}\right)^{2}, X_{1,0}^{-}\right]\right) \\
& =\left(q-q^{-1}\right) k_{1}^{-1}\left([2]_{q} k_{1} X_{1,2}^{+}+\left(q^{4}-q^{-2}\right)\left(X_{1,1}^{+}\left[X_{1,1}^{+}, X_{1,0}^{-}\right]+\left[X_{1,1}^{+}, X_{1,0}^{-}\right] X_{1,1}^{+}\right)\right) \\
& =\left(q-q^{-1}\right)[2]_{q} X_{1,2}^{+}+\left(q^{4}-q^{-2}\right)\left(q^{-2} X_{1,1}^{+} \tilde{H}_{1,1}^{+}+\tilde{H}_{1,1}^{+} X_{1,1}^{+}\right) \\
& =\left(q^{2}-q^{-2}\right) X_{1,2}^{+}+\left(q^{4}-q^{-2}\right) \tilde{H}_{1,1}^{+} X_{1,1}^{+}+\left(q^{2}-q^{-4}\right) X_{1,1}^{+} \tilde{H}_{1,1}^{+},
\end{aligned}
$$


hence, for all $s \in \mathbb{Z}$,

$$
\left[\tilde{H}_{1,2}^{+}, X_{1, s}^{+}\right]=\left(q^{2}-q^{-2}\right) X_{1, s+2}^{+}+\left(q^{4}-q^{-2}\right)\left[\tilde{H}_{1,1}^{+}, X_{1, s+1}^{+}\right]_{-q^{-2}} ;
$$

for $r>2$ :

$$
\begin{aligned}
& {\left[\tilde{H}_{1, r}^{+}, X_{1,0}^{+}\right]=\left(q-q^{-1}\right) k_{1}^{-1}\left([2]_{q} k_{1} X_{1, r}^{+}+\left[\left[X_{1, r}^{+}, X_{1,0}^{+}\right]_{q^{2}}, X_{1,0}^{-}\right]\right) } \\
&=\left(q-q^{-1}\right) k_{1}^{-1}\left([2]_{q} k_{1} X_{1, r}^{+}+\left[\left(q^{4}-q^{-2}\right)\left[X_{1, r-1}^{+}, X_{1,1}^{+}\right]_{-1}-\left[X_{1,2}^{+}, X_{1, r-2}^{+}\right]_{q^{2}}, X_{1,0}^{-}\right]\right) \\
&=\left(q-q^{-1}\right) k_{1}^{-1}\left([2]_{q} k_{1} X_{1, r}^{+}\right. \\
& \quad+\left(q^{4}-q^{-2}\right)\left[X_{1, r-1}^{+},\left[X_{1,1}^{+}, X_{1,0}^{-}\right]\right]_{-1}+\left(q^{4}-q^{-2}\right)\left[X_{1,1}^{+},\left[X_{1, r-1}^{+}, X_{1,0}^{-}\right]\right]_{-1} \\
&\left.\quad-\left[X_{1,2}^{+},\left[X_{1, r-2}^{+}, X_{1,0}^{-}\right]\right]_{q^{2}}+q^{2}\left[X_{1, r-2}^{+},\left[X_{1,2}^{+}, X_{1,0}^{-}\right]\right]_{q^{-2}}\right) \\
&=\left(q^{2}-q^{-2}\right) X_{1, r}^{+} \\
& \quad+\left(q^{4}-q^{-2}\right)\left[\tilde{H}_{1,1}^{+}, X_{1, r-1}^{+}\right]_{-q^{-2}}+\left(q^{4}-q^{-2}\right)\left[\tilde{H}_{1, r-1}^{+}, X_{1,1}^{+}\right]_{-q^{-2}} \\
&+q^{2}\left[\tilde{H}_{1, r-2}^{+}, X_{1,2}^{+}\right]_{q^{-4}}-\left[\tilde{H}_{1,2}^{+}, X_{1, r-2}^{+}\right] \\
&=\left(q^{4}-q^{-2}\right) \tilde{H}_{1, r-1}^{+} X_{1,1}^{+}+\left(q^{2}-q^{-4}\right) X_{1,1}^{+} \tilde{H}_{1, r-1}^{+} \\
&+q^{2} \tilde{H}_{1, r-2}^{+} X_{1,2}^{+}-q^{-2} X_{1,2}^{+} \tilde{H}_{1, r-2}^{+} ;
\end{aligned}
$$

this implies, using again $\left(H X L^{+}\right)$, that

$$
\begin{aligned}
\left({ }_{l} \tilde{H}_{1}^{+}(u)-{ }_{r} \tilde{H}_{1}^{+}(u)\right)\left(X_{1,0}^{+}\right)= & \left(\left(q^{4}-q^{-2}\right){ }_{l} \tilde{H}_{1}^{+}(u) \tilde{t}_{1}^{-1} u+\left(q^{2}-q^{-4}\right)_{r} \tilde{H}_{1}^{+}(u) \tilde{t}_{1}^{-1} u\right. \\
& \left.+q^{2}{ }_{l} \tilde{H}_{1}^{+}(u)\left(\tilde{t}_{1}^{-1} u\right)^{2}-q^{-2}{ }_{r} \tilde{H}_{1}^{+}(u)\left(\tilde{t}_{1}^{-1} u\right)^{2}\right)\left(X_{1,0}^{+}\right),
\end{aligned}
$$

or equivalently

${ }_{l} \tilde{H}_{1}^{+}(u)\left(1-q^{4} \tilde{t}_{1}^{-1} u\right)\left(1+q^{-2} \tilde{t}_{1}^{-1} u\right)\left(X_{1,0}^{+}\right)={ }_{r} \tilde{H}_{1}^{+}(u)\left(1-q^{-4} \tilde{t}_{1}^{-1} u\right)\left(1+q^{2} \tilde{t}_{1}^{-1} u\right)\left(X_{1,0}^{+}\right) ;$

from this we get

$$
\begin{aligned}
\left(q-q^{-1}\right)\left({ }_{l} H_{1}(u)-{ }_{r} H_{1}(u)\right)\left(X_{1,0}^{+}\right) & =\left(\log \left(1-q^{-4}\left(\tilde{t}_{1}^{-1} u\right)\right)+\log \left(1+q^{2}\left(\tilde{t}_{1}^{-1} u\right)\right)\right. \\
& \left.-\log \left(1-q^{4}\left(\tilde{t}_{1}^{-1} u\right)\right)-\log \left(1+q^{-2}\left(\tilde{t}_{1}^{-1} u\right)\right)\right)\left(X_{1,0}^{+}\right)
\end{aligned}
$$

that is,

$$
\left[H_{1, r}, X_{1,0}^{+}\right]=\frac{-q^{-4 r}+(-1)^{r-1} q^{2 r}+q^{4 r}-(-1)^{r-1} q^{-2 r}}{r\left(q-q^{-1}\right)} X_{1, r}^{+}=b_{11 r} X_{1, r}^{+} .
$$

Proposition 7.16. In $\tilde{\mathcal{U}}_{q}^{\mathrm{Dr}}$ we have $\mathcal{I}(H H) \subseteq \mathcal{I}(H X)$.

Proof. Thanks to Remark 6.3, to the fact that

$$
\left[H_{i, r}, H_{j, s}\right]=-\left[H_{j, s}, H_{i, r}\right]=\tilde{\Omega}\left[H_{j,-s}, H_{i,-r}\right]
$$

and to the definition of $b_{i j r}$, it is enough to prove that in $\tilde{\mathcal{U}}_{q}^{\mathrm{Dr}} / \mathcal{I}(H X)$,

$$
\left[H_{i, r}, \tilde{H}_{j, s}^{+}\right]=\delta_{r+s, 0} b_{i j r}\left(C^{r}-C^{-r}\right) \quad \text { if }|r| \geq s>0 .
$$


This is an easy computation:

$$
\begin{aligned}
& {\left[H_{i, r}, \tilde{H}_{j, s}^{+}\right]=\left(q_{j}-q_{j}^{-1}\right) k_{j}^{-1}\left[H_{i, r},\left[X_{j, s}^{+}, X_{j, 0}^{-}\right]\right] } \\
&=\left(q_{j}-q_{j}^{-1}\right) k_{j}^{-1}\left(\left[\left[H_{i, r}, X_{j, s}^{+}\right], X_{j, 0}^{-}\right]-\left[\left[H_{i, r}, X_{j, 0}^{-}\right], X_{j, s}^{+}\right]\right) \\
&=\left(q_{j}-q_{j}^{-1}\right) b_{i j r} k_{j}^{-1}\left(C^{\frac{r-|r|}{2}}\left[X_{j, r+s}^{+}, X_{j, 0}^{-}\right]-C^{\frac{r+|r|}{2}}\left[X_{j, s}^{+}, X_{j, r}^{-}\right]\right) \\
&=b_{i j r} k_{j}^{-1}\left(C^{\frac{r-|r|}{2}} k_{j} \tilde{H}_{j, r+s}^{+}-C^{\frac{-r-|r|}{2}-s} k_{j}^{-1} \tilde{H}_{j, r+s}^{-}\right. \\
&\left.-C^{\frac{-r+|r|}{2}} k_{j} \tilde{H}_{j, r+s}^{+}+C^{\frac{r+|r|}{2}-s} k_{j}^{-1} \tilde{H}_{j, r+s}^{-}\right) \\
&= b_{i j r} k_{j}^{-1}\left(\left(C^{\frac{r-|r|}{2}}-C^{\frac{-r+|r|}{2}}\right) k_{j} \tilde{H}_{j, r+s}^{+}+\left(C^{\frac{r+|r|}{2}}-C^{\frac{-r-|r|}{2}}\right) C^{-s} k_{j}^{-1} \tilde{H}_{j, r+s}^{-}\right) \\
&= b_{i j r} k_{j}^{-1}\left(C^{\frac{r-|r|}{2}}-C^{\frac{-r+|r|}{2}}\right) k_{j} \tilde{H}_{j, r+s}^{+}=\delta_{r+s, 0} b_{i j r}\left(C^{r}-C^{-r}\right) .
\end{aligned}
$$

Corollary 7.17. (i) The relations $(Z H)$ and $(K H)$ are redundant.

(ii) In $\tilde{\mathcal{U}}_{q}^{\text {Dr }}$ the relations $\left(H X^{ \pm}\right)$depend on $\left(X D^{ \pm}\right),\left(X 1^{ \pm}\right)$and $\left(X 2^{ \pm}\right)$, and the relations $(H H)$ depend on $(X D),(X 1)$ and $(X 2)$.

(iii) $\mathcal{U}_{q}^{\mathrm{Dr}}\left(X_{\tilde{n}}^{(k)}\right)$ is the quotient of $\tilde{\mathcal{U}}_{q}^{\mathrm{Dr}}\left(X_{\tilde{n}}^{(k)}\right)$ by the ideal generated by the relations $\left(X D^{ \pm}\right)-\left(S 3^{ \pm}\right)$.

Remark 7.18. It is worth remarking that Corollary 7.17(ii) allows us to reduce the relations $\left(H X^{ \pm}\right)$and $(H H)$ to relations involving just the $X_{i, r}^{ \pm}$, s, without using the $H_{i, r}$ 's whose connection with the $\tilde{H}_{i, r}^{ \pm}$'s (these last can be expressed in terms of commutators between the $X_{i, r}^{+}$'s and the $X_{i, r}^{-}$'s, see Remark 6.4) is complicated to handle. Indeed the relations $\left(H X L^{ \pm}\right)$can be translated as follows:

(i) if $\tilde{d}_{i} \leq|r|<\tilde{d}_{i j}$ then $\left[H_{i, r}, X_{j, s}^{ \pm}\right]=0$, that is, $X_{j, s}^{ \pm}$commutes with the subalgebra generated by $\left\{H_{i, r}\left|\tilde{d}_{i} \leq\right| r \mid<\tilde{d}_{i j}\right\}$, which is the subalgebra generated by $\left\{\tilde{H}_{i, r}^{ \pm}\left|\tilde{d}_{i} \leq\right| r \mid<\tilde{d}_{i j}\right\}$ (see Remark 6.3); hence these relations can be rewritten as

$$
\left[\tilde{H}_{i, r}^{ \pm}, X_{j, s}^{+}\right]=0 \quad \text { and } \quad\left[\tilde{H}_{i, r}^{ \pm}, X_{j, s}^{-}\right]=0 \quad \text { if }|r|<\tilde{d}_{i j}
$$

(ii) if $|r|=\tilde{d}_{i j}$ and $\pm r>0$ then $\tilde{H}_{i, r}^{ \pm} \mp\left(q_{i}-q_{i}^{-1}\right) H_{i, r}$ commutes with $X_{j, s}^{ \pm}$, by (i) and Remark 6.3, hence in the relations $\left[H_{i, r}, X_{j, s}^{ \pm}\right]= \pm b_{i j \tilde{d}_{i j}} C^{\frac{r \mp|r|}{2}} X_{j, r+s}^{ \pm}$we can replace $H_{i, r}$ with $\pm \tilde{H}_{i, r}^{ \pm} /\left(q_{i}-q_{i}^{-1}\right)$.

Thus the relations $\left(H X L^{ \pm}\right)$are equivalent to

$$
\begin{gathered}
{\left[\left[X_{i, \pm r}^{ \pm}, X_{i, 0}^{\mp}\right], X_{j, s}^{ \pm}\right]_{q_{i}^{a_{i j}}}=b_{i j r} k_{i}^{ \pm 1} X_{j, s \pm r}^{ \pm},} \\
{\left[\left[X_{i, \pm r}^{ \pm}, X_{i, 0}^{\mp}\right], X_{j, s}^{\mp}\right]_{q_{i}^{-} a_{i j}}=-b_{i j r} C^{ \pm r} k_{i}^{ \pm 1} X_{j, s \pm r}^{\mp}}
\end{gathered}
$$

with $0<r \leq \tilde{d}_{i j}$. 
Note also that among the relations defining $\mathcal{U}_{q}^{\mathrm{Dr}}$ there are no other relations involving the $H_{i, r}$ 's.

Remark 7.19. Note that for each $i \in I_{0},\left\{C^{ \pm \tilde{d}_{i}}, k_{i}^{ \pm 1}, X_{i, r}^{ \pm}, H_{i, s}\left|\tilde{d}_{i}\right| r, s ; s \neq 0\right\}$ generates $\operatorname{Im}\left(\tilde{\phi}_{i}\right) \subseteq \tilde{\mathcal{U}}_{q}^{\text {Dr }}$ over $\mathbb{C}\left(q_{i}\right)$. Therefore the following sets generate $\operatorname{Im}\left(\tilde{\phi}_{i}\right)$ (hence $\left.\operatorname{Im}\left(\phi_{i}\right) \subseteq \mathcal{U}_{q}^{\text {Dr }}\right)$ over $\mathbb{C}\left(q_{i}\right)$ :

(i) $\left\{C^{ \pm \tilde{d}_{i}}, k_{i}^{ \pm 1}, X_{i, r}^{ \pm}\left|\tilde{d}_{i}\right| r\right\}$;

(ii) $\left\{C^{ \pm \tilde{d}_{i}}, k_{i}^{ \pm 1}, X_{i, 0}^{ \pm}, H_{i, \pm \tilde{d}_{i}}\right\}$;

(iii) $\left\{C^{ \pm \tilde{d}_{i}}, k_{i}^{ \pm 1}, X_{i, 0}^{ \pm}, X_{i, \mp \tilde{d}_{i}}^{ \pm}\right\}$.

\section{Moreover}

(iv) $\left\{C^{ \pm 1}, k_{i}^{ \pm 1}, X_{i, 0}^{ \pm}, X_{i_{0}, \mp 1}^{ \pm} \mid i \in I_{0}\right\}$ (where $i_{0}$ is any fixed element of $I_{0}$ with $\tilde{d}_{i_{0}}=1$ ) generates $\tilde{\mathcal{U}}_{q}^{\text {Dr }}$ (hence $\mathcal{U}_{q}^{\text {Dr }}$ ) over $\mathbb{C}(q)$.

Proof. (i) See Remarks 6.3 and 6.4 .

(ii) follows from (i) by induction on $|r|$, using that

$$
\forall r \in \mathbb{Z} \quad\left[H_{i, \pm \tilde{d}_{i}}, X_{i, r}^{+}\right]=b_{i i \tilde{d}_{i}} C^{\frac{ \pm 1-1}{2} \tilde{d}_{i}} X_{i, r \pm \tilde{d}_{i}}^{+}
$$

and applying $\tilde{\Omega}$ (the set $\left\{C^{ \pm \tilde{d}_{i}}, k_{i}^{ \pm 1}, X_{i, 0}^{ \pm}, H_{i, \pm \tilde{d}_{i}}\right\}$ is $\tilde{\Omega}$-stable).

(iii) is an immediate consequence of (ii) and of the fact that $\left[X_{i, \tilde{d}_{i}}^{+}, X_{i, 0}^{-}\right]=$ $k_{i} H_{i, \tilde{d}_{i}}$, again applying $\tilde{\Omega}$.

(iv) For each $i \in I_{0}$ there exists a sequence of different indices $i_{0}, i_{1}, \ldots, i_{l}=i$ in $I_{0}$ such that $a_{i_{h-1} i_{h}}<0$ and $\tilde{d}_{i_{h-1}} \mid \tilde{d}_{i_{h}}$ for all $h=1, \ldots, l$.

We prove by induction on $h$ that $\operatorname{Im}\left(\phi_{i_{h}}\right)$ is contained in the $\mathbb{C}(q)$-subalgebra of $\tilde{\mathcal{U}}_{q}^{\text {Dr }}$ generated by $\left\{C^{ \pm 1}, k_{i}^{ \pm 1}, X_{i, 0}^{ \pm}, X_{i_{0}, \mp 1}^{ \pm} \mid i \in I_{0}\right\}$, the claim for $h=0$ being (iii).

For $h>0$ it is again enough to use (iii), noting that

$$
\left[H_{i_{h-1},-\tilde{d}_{i_{h}}}, X_{i_{h}, 0}^{+}\right]=b_{i_{h-1} i_{h} \tilde{d}_{i_{h}}} C^{-\tilde{d}_{i_{h}}} X_{i_{h},-\tilde{d}_{i_{h}}}^{+} \neq 0
$$

and applying $\tilde{\Omega}$.

\section{$\S 8 . \mathcal{U}_{q}:($ anti)automorphisms and relations}

The main point of this section is to describe in some detail how the (anti)automorphisms $\tilde{\Omega}, \tilde{\Theta}$ and $\tilde{t}_{i}\left(i \in I_{0}\right)$ act on the generators of the ideal of $\tilde{\mathcal{U}}_{q}$ defining $\mathcal{U}_{q}$. As a corollary, $\tilde{\Omega}, \tilde{\Theta}$ and $\tilde{t}_{i}\left(i \in I_{0}\right)$ induce analogous $\Omega, \Theta$ and $t_{i}\left(i \in I_{0}\right)$ on $\mathcal{U}_{q}$. But the important consequence of this analysis (together with the study of the commutation with the elements $H_{i, r}$ 's) is the reduction of the huge amount of 
the relations $\left(X D^{ \pm}\right)-\left(S 3^{ \pm}\right)$to relations involving only the positive $X_{i, r}^{+}$(which is obvious and well known) and, what is new, to the analogous relations with "constant parameters" (see §9). Lemmas 9.12 and 9.14 are the fundamental tool of this paper, which makes possible and easy the computations of the following sections, leading to Theorems 10.8 and 11.18 .

Notation 8.1. Let $l \in \mathbb{N}$; then:

(i) $\mathbb{1}=\mathbb{1}_{l}=(1, \ldots, 1) \in \mathbb{Z}^{l}$;

(ii) $\left\{e_{1}, \ldots, e_{l}\right\}$ is the canonical basis of $\mathbb{Z}^{l}$;

(iii) for all $r=\left(r_{1}, \ldots, r_{l}\right) \in \mathbb{Z}^{l}, \bar{r} \in \mathbb{Z}^{l}$ denotes $\bar{r}=\left(r_{l}, \ldots, r_{1}\right)$.

Definition 8.2. $\Omega: \mathcal{U}_{q}^{\mathrm{Dr}} \rightarrow \mathcal{U}_{q}^{\mathrm{Dr}}$ is the $\mathbb{C}$-antilinear antihomomorphism induced by $\tilde{\Omega}$ (and by $\bar{\Omega}$, see Definition 6.15 and Remark 6.16 (vii)), that is, the $\mathbb{C}$-antilinear antihomomorphism defined on the generators by

$$
q \mapsto q^{-1}, \quad C^{ \pm 1} \mapsto C^{\mp 1}, \quad k_{i}^{ \pm 1} \mapsto k_{i}^{\mp 1}, \quad X_{i, r}^{ \pm} \mapsto X_{i,-r}^{\mp}, \quad H_{i, r} \mapsto H_{i,-r} .
$$

Remark 8.3. $\Omega$ is a well-defined involution of $\mathcal{U}_{q}^{\mathrm{Dr}}$. Indeed

$$
\begin{aligned}
\tilde{\Omega}\left(M_{(2)}^{ \pm}((i, r),(j, s))\right) & =-q_{i}^{-a_{i j}} M_{(2)}^{\mp}((i,-r),(j,-s)), \\
\tilde{\Omega}\left(M_{i}^{ \pm}(r)\right) & =-q_{i}^{-2} M_{i}^{\mp}(-r), \\
\tilde{\Omega}\left(M_{(2,2)}^{ \pm}(r)\right) & =-q^{-2} M_{(2,2)}^{\mp}(-r), \\
\tilde{\Omega}\left(M_{(3)}^{\varepsilon, \pm}(r)\right) & =q^{-6 \varepsilon} M_{(3)}^{\varepsilon, \mp}(-r), \\
\tilde{\Omega}\left(M_{i, j ; l ; a}^{ \pm}(r ; s)\right) & =(-1)^{l} q_{i}^{l\left(a_{i j}+a(l-1)\right)} M_{i, j ; l ; a}^{\mp}(-r ;-s), \\
\tilde{\Omega}\left(X_{i, j ; l ; a}^{ \pm}(r ; s)\right) & =(-1)^{l} X_{i, j ; l ; a}^{\mp}(-\bar{r} ;-s), \\
\tilde{\Omega}\left(X_{[k]}^{ \pm}(r ; s)\right) & =X_{[k]}^{\mp}(-\bar{r} ;-s), \\
\tilde{\Omega}\left(M_{[2]}^{ \pm}(r ; s)\right) & =q^{-2} M_{[2]}^{\mp}(-r ;-s), \\
\tilde{\Omega}\left(M_{[3]}^{ \pm}(r ; s)\right) & =q^{-4} M_{[3]}^{\mp}(-r ;-s) .
\end{aligned}
$$

Definition 8.4. $\Theta: \mathcal{U}_{q}^{\mathrm{Dr}} \rightarrow \mathcal{U}_{q}^{\mathrm{Dr}}$ is the $\mathbb{C}$-antilinear homomorphism induced by $\tilde{\Theta}$ (and by $\bar{\Theta}$, see Definition 6.15 and Corollary 7.13(ii)), that is, the $\mathbb{C}$-antilinear homomorphism defined on the generators by

$$
\begin{aligned}
q \mapsto q^{-1}, & C^{ \pm 1} \mapsto C^{ \pm 1}, \quad k_{i}^{ \pm 1} \mapsto k_{i}^{\mp 1}, \\
X_{i, r}^{+} \mapsto-X_{i,-r}^{+} k_{i} C^{-r}, & X_{i, r}^{-} \mapsto-k_{i}^{-1} C^{-r} X_{i,-r}^{-}, \quad H_{i, r} \mapsto H_{i,-r} .
\end{aligned}
$$

Remark 8.5. $\Theta$ is a well-defined involution of $\mathcal{U}_{q}^{\mathrm{Dr}}$. Indeed

$$
\Theta_{\mathcal{F}}^{ \pm}\left(M_{(2)}^{ \pm}((i, r),(j, s))\right)=-q_{i}^{-a_{i j}} M_{(2)}^{ \pm}\left(\left(i,-r \mp \tilde{d}_{i j}\right),\left(j,-s \mp \tilde{d}_{i j}\right)\right),
$$




$$
\begin{aligned}
\Theta_{\mathcal{F}}^{ \pm}\left(M_{i}^{ \pm}(r)\right) & =-q_{i}^{-2} M_{i}^{ \pm}\left(-\bar{r} \mp \tilde{d}_{i j} \mathbb{1}\right), \\
\Theta_{\mathcal{F}}^{ \pm}\left(M_{(2,2)}^{ \pm}(r)\right) & =-q^{-2} M_{(2,2)}^{ \pm}(-\bar{r} \mp 2 \mathbb{1}), \\
\Theta_{\mathcal{F}}^{ \pm}\left(M_{(3)}^{\varepsilon, \pm}(r)\right) & =M_{(3)}^{-\varepsilon, \pm}(-r), \\
\Theta_{\mathcal{F}}^{ \pm}\left(X_{i, j ; l ; a}^{ \pm}(r ; s)\right) & =X_{i, j ; l ; a}^{ \pm}(-r ;-s), \\
\Theta_{\mathcal{F}}^{ \pm}\left(X_{[k]}^{ \pm}(r ; s)\right) & =X_{[k]}^{ \pm}(-r \mp(k-1) \mathbb{1} ;-s) .
\end{aligned}
$$

Definition 8.6. For all $i \in I_{0}, t_{i}: \mathcal{U}_{q}^{\text {Dr }} \rightarrow \mathcal{U}_{q}^{\text {Dr }}$ is the $\mathbb{C}(q)$-homomorphism induced by $\tilde{t}_{i}$ (and by $\bar{t}_{i}$, see Definition 6.15 and Corollary 7.10(ii)), that is, the $\mathbb{C}(q)$ homomorphism defined on the generators by

$$
C^{ \pm 1} \mapsto C^{ \pm 1}, \quad k_{j}^{ \pm 1} \mapsto\left(k_{j} C^{-\delta_{i j} \tilde{d}_{i}}\right)^{ \pm 1}, \quad X_{j, r}^{ \pm} \mapsto X_{j, r \mp \delta_{i j} \tilde{d}_{i}}^{ \pm}, \quad H_{j, r} \mapsto H_{j, r} .
$$

Remark 8.7. It is immediate to check that the $t_{i}$ 's are well-defined automorphisms of $\mathcal{U}_{q}^{\mathrm{Dr}}$. Indeed

$$
\begin{aligned}
\tilde{t}_{i}\left(M_{(2)}^{ \pm}((j, r),(h, s))\right) & =M_{(2)}^{ \pm}\left(\left(j, r \mp \delta_{i j} \tilde{d}_{i}\right),\left(h, s \mp \delta_{i h} \tilde{d}_{i}\right)\right), \\
\tilde{t}_{i}\left(M_{j}^{ \pm}(r)\right) & =M_{j}^{ \pm}\left(r \mp \delta_{i j} \tilde{d}_{i} \mathbb{1}\right), \\
\tilde{t}_{i}\left(M_{(2,2)}^{ \pm}(r)\right) & =M_{(2,2)}^{ \pm}\left(r \mp \delta_{i 1} \mathbb{1}\right), \\
\tilde{t}_{i}\left(M_{(3)}^{\varepsilon, \pm}(r)\right) & =M_{(3)}^{\varepsilon, \pm}\left(r \mp \delta_{i 1} \mathbb{1}\right), \\
\tilde{t_{i}}\left(M_{j, h ; l ; a}^{ \pm}(r ; s)\right) & =M_{j, h ; l ; a}^{ \pm}\left(r \mp \delta_{i j} \tilde{d}_{i} \mathbb{1} ; s \mp \delta_{i h} \tilde{d}_{i}\right), \\
\tilde{t}_{i}\left(X_{j, h ; l ; a}^{ \pm}(r ; s)\right) & =X_{j, h ; l ; a}^{ \pm}\left(r \mp \delta_{i j} \tilde{d}_{i} \mathbb{1} ; s \mp \delta_{i h} \tilde{d}_{i}\right), \\
\tilde{t}_{i}\left(X_{[k]}^{ \pm}(r ; s)\right) & =X_{[k]}^{ \pm}\left(r \mp \delta_{i j} \mathbb{1} ; s \mp \delta_{i h} \tilde{d}\right), \\
\tilde{t}_{i}\left(M_{[k]}^{ \pm}(r ; s)\right) & =M_{[k]}^{ \pm}\left(r \mp \delta_{i j} \mathbb{1} ; s \mp \delta_{i h} k\right) ;
\end{aligned}
$$

in the last two identities, $j, h \in I_{0}$ are such that $a_{j h}=-k$.

Remark 8.8. Of course (see Remark 6.16(v)\&(vi))

$$
\Theta \Omega=\Omega \Theta, \quad t_{i} \Omega=\Omega t_{i}, \quad t_{i} \Theta=\Theta t_{i}^{-1}, \quad t_{i} t_{j}=t_{j} t_{i} \quad \forall i, j \in I_{0}
$$

as maps of $\mathcal{U}_{q}^{\operatorname{Dr}}\left(X_{\tilde{n}}^{(k)}\right)$ into itself, and, for all $i \in I_{0}$,

$$
\Omega \phi_{i}=\phi_{i} \Omega, \quad \Theta \phi_{i}=\phi_{i} \Theta, \quad t_{i} \phi_{i}=\phi_{i} t_{1}, \quad t_{j} \phi_{i}=\phi_{i} \quad \forall j \in I_{0} \backslash\{i\}
$$

as maps from $\mathcal{U}_{q}^{\operatorname{Dr}}\left(A_{1}^{(1)}\right)$ to $\mathcal{U}_{q}^{\operatorname{Dr}}\left(X_{\tilde{n}}^{(k)}\right)$ if $\left(X_{\tilde{n}}^{(k)}, i\right) \neq\left(A_{2 n}^{(2)}, 1\right)$, and from $\mathcal{U}_{q}^{\operatorname{Dr}}\left(A_{2}^{(2)}\right)$ to $\mathcal{U}_{q}^{\operatorname{Dr}}\left(X_{\tilde{n}}^{(k)}\right)$ if $\left(X_{\tilde{n}}^{(k)}, i\right)=\left(A_{2 n}^{(2)}, 1\right)$.

Moreover, for all $\alpha \in Q, \beta \in Q_{0}, m \in \mathbb{Z}, i \in I_{0}$ we have

$$
\Omega\left(\mathcal{U}_{q, \alpha}^{\mathrm{Dr}}\right)=\mathcal{U}_{q,-\alpha}^{\mathrm{Dr}}, \quad \Theta\left(\mathcal{U}_{q, \beta+m \delta}^{\mathrm{Dr}}\right)=\mathcal{U}_{q, \beta-m \delta}^{\mathrm{Dr}}, \quad t_{i}\left(\mathcal{U}_{q, \alpha}^{\mathrm{Dr}}\right)=\mathcal{U}_{q, \lambda_{i}(\alpha)}^{\mathrm{Dr}} .
$$




\section{§. Reduction to relations with constant parameter}

We shall now apply the structures introduced until now to the analysis of the relations defining $\mathcal{U}_{q}^{\mathrm{Dr}}$.

Notation 9.1. Let $(R)$ be relations as in Notation 5.1 and define the following ideals of $\mathcal{U}$ :

$$
\mathcal{I}_{\text {const }}(R)=\left(S_{\zeta}\left(r \mathbb{1}_{l}, s\right) \mid \zeta \in \mathcal{Z}, r \in \mathbb{Z}, s \in \mathbb{Z}^{\tilde{l}}\right), \quad \mathcal{I}_{0}(R)=\left(S_{\zeta}(\underline{0}) \mid \zeta \in \mathcal{Z}\right),
$$

where $\underline{0} \in \mathbb{Z}^{l+\tilde{l}}$; more precisely, if $\# \mathcal{Z}=1$ and $\mathcal{Z}=\{\zeta\}$, then given $r \in \mathbb{Z}$ and $s \in \mathbb{Z}^{\tilde{l}}$, let

$$
\mathcal{I}_{(r, s)}(R)\left(=\mathcal{I}_{(r, s)}\left(R_{\zeta}\right)\right)=\left(S_{\zeta}\left(r \mathbb{1}_{l}, s\right)\right)
$$

If $\left({ }^{(h)} R\right)(h=1, \ldots, m)$ are as in Notation 5.1(iii), define

$$
\begin{aligned}
\mathcal{I}_{\text {const }}\left({ }^{(1)} R, \ldots,{ }^{(m)} R\right) & =\left(\mathcal{I}_{\text {const }}\left({ }^{(1)} R\right), \ldots, \mathcal{I}_{\text {const }}\left({ }^{(m)} R\right)\right), \\
\mathcal{I}_{0}\left({ }^{(1)} R, \ldots,{ }^{(m)} R\right) & =\left(\mathcal{I}_{0}\left({ }^{(1)} R\right), \ldots, \mathcal{I}_{0}\left({ }^{(m)} R\right)\right) .
\end{aligned}
$$

Finally, if moreover each $\left({ }^{(h)} R\right)$ is $\left({ }^{(h)} R^{+}\right) \cup\left({ }^{(h)} R^{-}\right)$where $\left({ }^{(h)} R^{ \pm}\right)$is as in Notation $5.1(\mathrm{iv})$, we shall also use the notation

$$
\mathcal{I}_{*}^{ \pm}\left({ }^{(1)} R, \ldots,{ }^{(m)} R\right)=\left(\mathcal{I}_{*}\left({ }^{(1)} R^{ \pm}\right), \ldots, \mathcal{I}_{*}\left({ }^{(m)} R^{ \pm}\right)\right)
$$

where $* \in\{\emptyset$, const, 0$\}$.

Remark 9.2. With the notation fixed in Notations 5.1 and 9.1 we have:

(i) $\mathcal{I}_{0}(R) \subseteq \mathcal{I}_{\text {const }}(R) \subseteq \mathcal{I}(R)$;

(ii) $\mathcal{I}_{*}(R)=\left(\mathcal{I}_{*}\left(R_{\zeta}\right) \mid \zeta \in \mathcal{Z}\right)$ for each $* \in\{\emptyset$, const, 0$\}$;

(iii) for all $\zeta \in \mathcal{Z}, \mathcal{I}_{\text {const }}\left(R_{\zeta}\right)=\left(\mathcal{I}_{(r, s)}\left(R_{\zeta}\right) \mid(r, s) \in \mathbb{Z}^{1+\tilde{l}}\right)$;

(iv) $l=1 \Rightarrow \mathcal{I}_{\text {const }}(R)=\mathcal{I}(R)$.

Remark 9.3. Let $\left(R^{ \pm}\right)$be relations in $\tilde{\mathcal{U}}_{q}^{\text {Dr }}$ as in Notation 5.1 (iv) and suppose that for all $\zeta \in \mathcal{Z}, r \in \mathbb{Z}^{l}, s \in \mathbb{Z}^{\tilde{l}}$ there exists an invertible element $u_{\zeta, r, s}$ of $\tilde{\mathcal{U}}_{q}^{\mathrm{Dr}}$ such that $\tilde{\Omega}\left(S_{\zeta}^{+}(r, s)\right)=u_{\zeta, r, s} S_{\zeta}^{-}(-r,-s)$ (notice that if $\left(R^{ \pm}\right)$has this property then so does $\left.\left(R_{\zeta}^{ \pm}\right)\right)$. With the notation fixed in Notations 5.1 and 9.1 we have:

(i) $\tilde{\Omega}\left(\mathcal{I}_{r, s}^{+}\left(R_{\zeta}\right)\right)=\mathcal{I}_{-r,-s}^{-}\left(R_{\zeta}\right)$ for all $\zeta \in \mathcal{Z},(r, s) \in \mathbb{Z}^{1+\tilde{l}}$

(ii) $\tilde{\Omega}\left(\mathcal{I}^{+}(R)\right)=\mathcal{I}^{-}(R), \tilde{\Omega}\left(\mathcal{I}_{\text {const }}^{+}(R)\right)=\mathcal{I}_{\text {const }}^{-}(R)$ and $\tilde{\Omega}\left(\mathcal{I}_{0}^{+}(R)\right)=\mathcal{I}_{0}^{-}(R)$;

(iii) $\mathcal{I}(R), \mathcal{I}_{\text {const }}(R)$ and $\mathcal{I}_{0}(R)$ are the $\tilde{\Omega}$-stable ideals generated respectively by $\mathcal{I}^{+}(R), \mathcal{I}_{\text {const }}^{+}(R)$ and $\mathcal{I}_{0}^{+}(R)$. 
Remark 9.4. Let $(R)$ be relations in $\tilde{\mathcal{U}}_{q}^{\mathrm{Dr}}$ as in Notation 5.1 and suppose that for all $\zeta \in \mathcal{Z}$ there exist $i, j \in I_{0}$ such that for all $r \in \mathbb{Z}^{l}, s \in \mathbb{Z}^{\tilde{l}}$ we have:

$$
\begin{aligned}
\tilde{t}_{i}\left(S_{\zeta}(r, s)\right) & =S_{\zeta}\left(r-\mathbb{1}_{l}, s\right) \\
\tilde{t}_{j}\left(S_{\zeta}(r, s)\right) & =S_{\zeta}\left(r, s-\mathbb{1}_{\tilde{l}}\right) \\
\tilde{t}_{h}\left(S_{\zeta}(r, s)\right) & =S_{\zeta}(r, s) \quad \forall h \neq i, j
\end{aligned}
$$

Then:

(i) $\mathcal{I}\left(R_{\zeta}\right)$ and $\mathcal{I}_{\text {const }}\left(R_{\zeta}\right)$ are $\tilde{t}_{i}^{ \pm 1}$-stable for all $\zeta \in \mathcal{Z}$ and $i \in I_{0}$;

(ii) for all $\zeta \in \mathcal{Z}$ and $(r, s),(\tilde{r}, \tilde{s}) \in \mathbb{Z}^{1+\tilde{l}}$ there exists $\tilde{t} \in\left\langle\tilde{t}_{i} \mid i \in I_{0}\right\rangle$ such that $\mathcal{I}_{(\tilde{r}, \tilde{s})}\left(R_{\zeta}\right)=\tilde{t}\left(\mathcal{I}_{(r, s)}\left(R_{\zeta}\right)\right)$;

(iii) for all $\zeta \in \mathcal{Z}$ and $(r, s) \in \mathbb{Z}^{1+\tilde{l}}, \mathcal{I}_{\text {const }}\left(R_{\zeta}\right)$ is the $\tilde{t}_{i}^{ \pm 1}$-stable (for all $i \in I_{0}$ ) ideal generated by $\mathcal{I}_{(r, s)}\left(R_{\zeta}\right)$;

(iv) $\mathcal{I}_{\text {const }}(R)$ is the $\tilde{t}_{i}^{ \pm 1}$-stable ideal (for all $i \in I_{0}$ ) generated by $\mathcal{I}_{0}(R)$.

Corollary 9.5. (i) If $(R)$ satisfies the conditions of Remarks 9.3 and 9.4 then $\mathcal{I}_{\text {const }}(R)$ is the $\tilde{\Omega}$-stable and $\tilde{t}_{i}$-stable (for all $i \in I_{0}$ ) ideal generated by $\mathcal{I}_{0}^{+}(R)$. More precisely for each $\zeta \in \mathcal{Z}, \mathcal{I}_{\text {const }}\left(R_{\zeta}\right)$ is the $\tilde{\Omega}$-stable and $\tilde{t}_{i}$-stable (for all $\left.i \in I_{0}\right)$ ideal generated by $\mathcal{I}_{0}^{+}\left(R_{\zeta}\right)$.

(ii) Let ${ }^{\left({ }^{(1)} R^{ \pm}\right)}$and ${ }^{\left({ }^{(2)} R^{ \pm}\right)}$be as in Remarks 9.3 and 9.4 , and suppose that $\mathcal{I}_{0}^{+}\left({ }^{(1)} R\right) \subseteq \mathcal{I}_{\text {const }}^{+}\left({ }^{(2)} R\right)$; then $\mathcal{I}_{\text {const }}\left({ }^{(1)} R\right) \subseteq \mathcal{I}_{\text {const }}\left({ }^{(2)} R\right)$.

More precisely $\mathcal{I}_{\text {const }}^{+}\left({ }^{(1)} R\right) \subseteq \mathcal{I}_{\text {const }}^{+}\left({ }^{(2)} R\right)$ if and only if for all $\zeta \in{ }^{(1)} \mathcal{Z}$ there exists $(r, s) \in \mathbb{Z} \times \mathbb{Z}^{\tilde{l}_{h}}$ such that $\mathcal{I}_{(r, s)}^{+}\left({ }^{(1)} R_{\zeta}\right) \subseteq \mathcal{I}_{\text {const }}^{+}\left({ }^{(2)} R\right)$, and if this is the case we have also $\mathcal{I}_{\text {const }}^{-}\left({ }^{(1)} R\right) \subseteq \mathcal{I}_{\text {const }}^{-}\left({ }^{(2)} R\right)$.

Remark 9.6. With the notation fixed in Notation 5.1 suppose that

$$
\sigma . S_{\zeta}(r, s)=S_{\zeta}(r, s) \quad \forall \zeta \in \mathcal{Z}, r \in \mathbb{Z}^{l}, s \in \mathbb{Z}^{\tilde{l}}, \sigma \in \mathcal{S}_{l},
$$

where $\sigma . S_{\zeta}(r, s)=S_{\zeta}(\sigma . r, s)$ (see Notation 4.1(iv).

This condition is equivalent to the existence of elements $N_{\zeta}(r, s) \in \tilde{\mathcal{U}}_{q}^{\mathrm{Dr}}$ such that

$$
S_{\zeta}(r, s)=\sum_{\sigma \in \mathcal{S}_{l}} \sigma \cdot N_{\zeta}(r, s)
$$

Notice that in general the elements $N_{\zeta}(r, s)\left(r \in \mathbb{Z}^{l}, s \in \mathbb{Z}^{\tilde{l}}\right)$ are not uniquely determined by the $S_{\zeta}(r, s)$ 's.

But $N_{\zeta}\left(r \mathbb{1}_{l}, s\right)=\frac{1}{l !} S_{\zeta}\left(r \mathbb{1}_{l}, s\right)$ for all $(r, s) \in \mathbb{Z} \times \mathbb{Z}^{\tilde{l}}$ 
Remark 9.7. In the hypotheses of Remark 9.6 suppose that:

(i) $\mathcal{Z} \subseteq \begin{cases}I_{0} & \text { if } \tilde{l}=0, \\ \left\{(i, j) \in I_{0} \times I_{0} \mid i \neq j\right\} & \text { if } \tilde{l}=1 ;\end{cases}$

(ii) if $\tilde{l}=0$ and $i \in \mathcal{Z} \subseteq I_{0}$ there exists $c_{p, \sigma} \in \mathbb{C}(q)\left(p \in \mathbb{Z}^{l}, \sigma \in \mathcal{S}_{l}\right)$ such that for all $r \in \mathbb{Z}^{l}$,

$$
N_{i}(r)=\sum_{\substack{p \in \mathbb{Z}^{l} \\ \sigma \in \mathcal{S}_{l}}} c_{p, \sigma} X_{i, \tilde{d}_{i}\left(r_{\sigma(1)}+p_{1}\right)}^{+} \cdot \ldots \cdot X_{i, \tilde{d}_{i}\left(r_{\sigma(l)}+p_{l}\right)}^{+}
$$

(iii) if $\tilde{l}=1$ and $(i, j) \in \mathcal{Z} \subseteq I_{0} \times I_{0}$ there exists $\tilde{c}_{p, \sigma, u} \in \mathbb{C}(q)\left(p \in \mathbb{Z}^{l}, \sigma \in \mathcal{S}_{l}\right.$, $u \in\{0, \ldots, l\})$ such that for all $(r, s) \in \mathbb{Z}^{l} \times \mathbb{Z}^{\tilde{l}}$,

$$
\begin{aligned}
N_{(i, j)}(r, s)= & \sum_{\substack{p \in \mathbb{Z}^{l}, \sigma \in \mathcal{S}_{l} \\
u=0, \ldots, l}} \tilde{c}_{p, \sigma, u} X_{i, \tilde{d}_{i}\left(r_{\sigma(1)}+p_{1}\right)}^{+} \ldots X_{i, \tilde{d}_{i}\left(r_{\sigma(u)}+p_{u}\right)}^{+} X_{j, \tilde{d}_{j} s}^{+} \\
& \cdot X_{i, \tilde{d}_{i}\left(r_{\sigma(u+1)}+p_{u+1}\right)}^{+} \ldots X_{i, \tilde{d}_{i}\left(r_{\sigma(l)}+p_{l}\right)}^{+} .
\end{aligned}
$$

Then the conditions of Remark 9.4 are satisfied.

Remark 9.8. The relations $\left(X D^{ \pm}\right)-\left(S 3^{ \pm}\right)$, as well as $\left(T k^{ \pm}\right)$and $\left(S^{ \pm}\right)$, are of the form described in Remark 9.7 and satisfy the hypotheses of Remark 9.3, so that they all satisfy the conditions of Remarks 9.3 and 9.4 and in particular the properties stated in Corollary 9.5(i).

Remark 9.9. If the relations $(R)$ are of the form described in Remarks 9.6 and 9.7 we find that for all $h \in I_{0}$ and $p \in \mathbb{Z}_{+}$:

$$
\begin{aligned}
{\left[H_{h, p}, S_{i}(r)\right] } & =b_{h i p} \sum_{u=1}^{l} S_{i}\left(r+\frac{p}{\tilde{d}_{i}} e_{u}\right) \quad \text { if } \tilde{l}=0, \\
{\left[H_{h, p}, S_{(i, j)}(r, s)\right] } & =b_{h j p} S_{(i, j)}\left(r, s+\frac{p}{\tilde{d}_{j}}\right)+b_{h i p} \sum_{u=1}^{l} S_{(i, j)}\left(r+\frac{p}{\tilde{d}_{i}} e_{u}, s\right) \quad \text { if } \tilde{l}=1,
\end{aligned}
$$

where $S_{\zeta}(r, s)=0$ if $(r, s) \notin \mathbb{Z}^{l+\tilde{l}}$.

Our next goal is studying the ideals $\mathcal{I}^{ \pm}(R)$ and $\mathcal{I}(R)$ (see Notations 5.1 and 9.1), providing a set of generators smaller and simpler than all of $\left\{S_{\zeta}^{ \pm}(r, s) \mid \zeta \in \mathcal{Z}\right.$, $\left.r \in \mathbb{Z}^{l}, s \in \mathbb{Z}^{\tilde{l}}\right\}$. More precisely we shall show that under suitable hypotheses (fulfilled by the relations defining $\mathcal{U}_{q}^{\mathrm{Dr}}$ over $\tilde{\mathcal{U}}_{q}^{\text {Dr }}$ ) we have $\mathcal{I}^{ \pm}(R)=\mathcal{I}_{\text {const }}^{ \pm}(R)$.

Remark 9.10. The relations $\left(X D^{+}\right)$satisfy the conditions of Remark 9.2(iv), hence in particular $\mathcal{I}^{+}(X D)=\mathcal{I}_{\text {const }}^{+}(X D)$ and $\mathcal{I}(X D)=\mathcal{I}_{\text {const }}(X D)$ (see Remarks 9.3 and 9.8). 
We shall generalize in two steps this result for $\left(X D^{ \pm}\right)$to relations $(R)$ satisfying the properties described in Remarks 9.3 and 9.7 : the cases $l=2, \tilde{l}=0$ (in particular $\left(X 1^{ \pm}\right)$and $\left.\left(X 2^{ \pm}\right)\right)$will follow from Lemma 9.12, while the general case will be an application of Lemma 9.14.

Note that if we considered $\tilde{\mathcal{U}}_{q}^{\mathrm{Dr}} / \mathcal{I}(H X)$ instead of $\tilde{\mathcal{U}}_{q}^{\mathrm{Dr}}$ we would not need to deal with the two cases separately, but the result would follow in both cases from Lemma 9.14.

For the next remark recall Notation 8.1.

Remark 9.11. Consider an algebra $\mathcal{U}$ over a field of characteristic 0 , an automorphism $t$ of $\mathcal{U}$, and elements $z, N(r) \in \mathcal{U}\left(r \in \mathbb{Z}^{2}\right)$ such that:

(i) $t(N(r))=N(r+\mathbb{1})$ for all $r \in \mathbb{Z}^{2}$;

(ii) $[z, N(r)]=N\left(r+e_{1}\right)+N\left(r+e_{2}\right)=N\left(r_{1}+1, r_{2}\right)+N\left(r_{1}, r_{2}+1\right)$ for all $r=\left(r_{1}, r_{2}\right) \in \mathbb{Z}^{2}$.

If we put $S(r)=\sum_{\sigma \in \mathcal{S}_{2}} N(\sigma(r))$ then of course:

(a) $S(r)=S(\bar{r})$ for all $r \in \mathbb{Z}^{2}$;

(b) $S(r)$ satisfies (i) and (ii);

(c) $S(0)=2 N(0)$.

Lemma 9.12. Let $\mathcal{U}, t, z, N(r), S(r)$ be as in Remark 9.11. If $N(0)=0$ then $S(r)=0$ for all $r \in \mathbb{Z}^{l}$.

Proof. First of all Remark 9.11 implies that it is enough to prove that $S(0, r)=0$ for all $r \in \mathbb{N}$ : indeed (a) of Remark 9.11 implies that one can suppose $r_{1} \leq r_{2}$; moreover applying $t^{-r_{1}}$ one reduces to the case $r_{1}=0$.

Let us proceed by induction on $r$ : if $r=0$ the claim is true by hypothesis; let $r>0$; then by the inductive hypothesis $S(0, r-1)=0$ and $0=[z, S(0, r-1)]=$ $S(1, r-1)+S(0, r)$; if $r=1$ then $S(1, r-1)+S(0, r)=S(1,0)+S(0,1)=2 S(0,1)$, so that $S(0,1)=0$; if $r>1$ then $S(1, r-1)=t(S(0, r-2))=0$ by the inductive hypothesis, so that also $S(0, r)=0$.

Remark 9.13. Consider an algebra $\mathcal{U}$ over a field of characteristic 0 , an automorphism $t$ of $\mathcal{U}$, elements $z_{m}, N_{y}(r) \in \mathcal{U}\left(m \in \mathbb{Z}_{+}, y \in \mathcal{U}, r \in \mathbb{Z}^{l}\right.$ with $l \in \mathbb{Z}_{+}$ fixed) such that:

(i) $t\left(N_{y}(r)\right)=N_{y}(r+\mathbb{1})$ for all $y \in \mathcal{U}, r \in \mathbb{Z}^{l}$;

(ii) $\left[z_{m}, N_{y}(r)\right]=N_{\left[z_{m}, y\right]}(r)+\sum_{u=1}^{l} N_{y}\left(r+m e_{u}\right)$.

If we put $S_{y}(r)=\sum_{\sigma \in \mathcal{S}_{l}} N_{y}(\sigma(r))$ then of course:

(a) $S_{y}(r)=S_{y}(\sigma(r))$ for all $\sigma \in \mathcal{S}_{l}$; 
(b) $S_{y}(r)$ satisfies (ii) and (iii);

(c) $S_{y}(0)=l ! N_{y}(0)$.

Lemma 9.14. Let $\mathcal{U}, t, z_{m}, N_{y}(r), S_{y}(r)$ be as in Remark 9.13 and let $Y \subseteq \mathcal{U}$ be a subset such that $\left[z_{m}, Y\right] \subseteq Y$ for all $m \in \mathbb{Z}_{+}$. If $N_{y}(0)=0$ for all $y \in Y$ then $S_{y}(r)=0$ for all $y \in Y$ and $r \in \mathbb{Z}^{l}$.

Proof. First of all Remark 9.13 implies that it is enough to prove that $S_{y}(r)=0$ for all $y \in Y$ and $r=\left(r_{1}, \ldots, r_{l}\right) \in \mathbb{Z}^{l}$ such that $0=r_{1} \leq \cdots \leq r_{l}$ : indeed (a) of Remark 9.13 implies that one can suppose $r_{1} \leq \cdots \leq r_{l}$; moreover applying $t^{-r_{1}}$ one reduces to the case $r_{1}=0$.

Let $v=\max \left\{u=1, \ldots, l \mid r_{u}=0\right\}$ and proceed by induction on $v$ : if $v=l$ then $r=0$ and the claim is true by hypothesis; let $v<l$ and choose $m=r_{v+1}$; then

$$
\begin{aligned}
\max \left\{u=1, \ldots, l \mid\left(r-m e_{v+1}\right)_{u}\right. & =0\}=v+1 \\
\max \left\{u=1, \ldots, l \mid\left(r-m e_{v+1}+m e_{w}\right)_{u}\right. & =0\}=v+1 \quad \forall w>v+1,
\end{aligned}
$$

hence by the inductive hypothesis $S_{y}\left(r-m e_{v+1}\right)=0$ and $S_{y}\left(r-m e_{v+1}+m e_{w}\right)=0$ for all $y \in Y$ and $w>v+1$; it follows that

$$
\begin{aligned}
0 & =\left[z_{m}, S_{y}\left(r-m e_{v+1}\right)\right]=S_{\left[z_{m}, y\right]}\left(r-m e_{v+1}\right)+\sum_{w=1}^{l} S_{y}\left(r-m e_{v+1}+m e_{w}\right) \\
& =\sum_{w=1}^{v+1} S_{y}\left(r-m e_{v+1}+m e_{w}\right)=(v+1) S_{y}(r) .
\end{aligned}
$$

Proposition 9.15. Consider the notation fixed in Notation 5.1 and suppose that $\left(R^{+}\right)$satisfies the hypotheses of Remark 9.7. Then:

(i) if $l=2$ and $\tilde{l}=0$ we have $\mathcal{I}^{+}(R)=\mathcal{I}_{\text {const }}^{+}(R)$ in $\tilde{\mathcal{U}}_{q}^{\mathrm{Dr}}\left(X_{\tilde{n}}^{(k)}\right)$;

(ii) in any case $\mathcal{I}^{+}(R)=\mathcal{I}_{\text {const }}^{+}(R)$ in $\tilde{\mathcal{U}}_{q}^{\mathrm{Dr}}\left(X_{\tilde{n}}^{(k)}\right) / \mathcal{I}\left(\mathcal{H} \mathcal{X}^{+}\right)$;

(iii) if moreover the hypotheses of Remark 9.3 are satisfied then we also have $\mathcal{I}^{-}(R)=\mathcal{I}_{\text {const }}^{-}(R)\left(\right.$ in $\tilde{\mathcal{U}}_{q}^{\mathrm{Dr}}\left(X_{\tilde{n}}^{(k)}\right)$ and in $\tilde{\mathcal{U}}_{q}^{\mathrm{Dr}}\left(X_{\tilde{n}}^{(k)}\right) / \mathcal{I}\left(H X^{-}\right)$respectively $)$.

Proof. Let $\zeta \in \mathcal{Z}$; thanks to Remarks $9.4\left(\right.$ iv) and 7.9, for all $i \in I_{0}, \tilde{t}_{i}$ induces an automorphism $t_{i}^{\prime}$ of $\tilde{\mathcal{U}}_{q}^{\mathrm{Dr}}\left(X_{\tilde{n}}^{(k)}\right) / \mathcal{I}_{\text {const }}^{+}\left(R_{\zeta}\right)$ and of $\tilde{\mathcal{U}}_{q}^{\mathrm{Dr}}\left(X_{\tilde{n}}^{(k)}\right) / \mathcal{I}_{\text {const }}^{+}\left(H X, R_{\zeta}\right)$.

(i) Fix $i \in \mathcal{Z} \subseteq I_{0}$, and notice that the data

$$
\mathcal{U}=\tilde{\mathcal{U}}_{q}^{\mathrm{Dr}} / \mathcal{I}_{\text {const }}^{+}\left(R_{i}\right), \quad t=t_{i}^{\prime-1}, \quad z=\frac{1}{b_{i i \tilde{d}_{i}}} H_{\left(i, \tilde{d}_{i}\right)}, \quad N(r)=N_{i}(r) \quad\left(r \in \mathbb{Z}^{2}\right)
$$

satisfy the conditions of Remark 9.11 and Lemma 9.12. 
Since $\mathcal{I}_{0}^{+}\left(R_{i}\right)=0$ in $\tilde{\mathcal{U}}_{q}^{\mathrm{Dr}} / \mathcal{I}_{\text {const }}^{+}\left(R_{i}\right)$, Lemma 9.12 implies that $\mathcal{I}^{+}\left(R_{i}\right)=0$ in $\tilde{\mathcal{U}}_{q}^{\mathrm{Dr}} / \mathcal{I}_{\text {const }}^{+}\left(R_{i}\right)$, or equivalently that $\mathcal{I}^{+}\left(R_{i}\right) \subseteq \mathcal{I}_{\text {const }}^{+}\left(R_{i}\right)$ in $\tilde{\mathcal{U}}_{q}^{\mathrm{Dr}}$, and this for all $i \in I_{0} \subseteq \mathcal{Z}$, so that $\mathcal{I}^{+}(R)=\mathcal{I}_{\text {const }}^{+}(R)$ thanks to Remark $9.2(\mathrm{i}) \&(\mathrm{ii})$.

(ii) Fix

$$
\zeta= \begin{cases}i \in \mathcal{Z} \subseteq I_{0} & \text { if } \tilde{l}=0, \\ (i, j) \in \mathcal{Z} \subseteq I_{0} \times I_{0} & \text { if } \tilde{l}=1,\end{cases}
$$

and notice that the data

$$
\begin{aligned}
& \mathcal{U}=\tilde{\mathcal{U}}_{q}^{\mathrm{Dr}} / \mathcal{I}_{\text {const }}^{+}\left(H X, R_{\zeta}\right), \quad t=t_{i}^{\prime-1}, \quad z_{m}=\frac{1}{b_{i i \tilde{d}_{i} m}} H_{\left(i, \tilde{d}_{i} m\right)} \quad \forall m \in \mathbb{Z}_{+}, \\
& Y= \begin{cases}\{0,1\} & \text { if } \tilde{l}=0, \\
\left\{a X_{j, \tilde{d}_{j} s}^{+} \mid s \in \mathbb{Z}, a \in \mathbb{C}(q)\right\} & \text { if } \tilde{l}=1,\end{cases}
\end{aligned}
$$

and for $r \in \mathbb{Z}^{l}$

$$
N_{y}(r)= \begin{cases}y N_{i}^{+}(r) & \text { if } \tilde{l}=0 \\ a N_{(i, j)}^{+}(r, s) & \text { if } \tilde{l}=1, y=a X_{j, \tilde{d}_{j} s}^{+},\end{cases}
$$

satisfy the conditions of Remark 9.13 and Lemma 9.14.

Since $\mathcal{I}_{0}^{+}\left(R_{i}\right)=0$ in $\tilde{\mathcal{U}}_{q}^{\mathrm{Dr}} / \mathcal{I}_{\text {const }}^{+}\left(H X, R_{i}\right)$ if $\tilde{l}=0$, and $\mathcal{I}_{0, s}^{+}\left(R_{(i, j)}\right)=0$ in $\tilde{\mathcal{U}}_{q}^{\mathrm{Dr}} / \mathcal{I}_{\text {const }}^{+}\left(H X, R_{(i, j)}\right)$ for all $s \in \mathbb{Z}$ if $\tilde{l}=1$, Lemma 9.14 implies that $\mathcal{I}^{+}\left(R_{\zeta}\right)=0$ in $\tilde{\mathcal{U}}_{q}^{\mathrm{Dr}} / \mathcal{I}_{\text {const }}^{+}\left(H X, R_{\zeta}\right)$, or equivalently $\mathcal{I}^{+}\left(R_{\zeta}\right) \subseteq \mathcal{I}_{\text {const }}^{+}\left(R_{\zeta}\right)$ in $\tilde{\mathcal{U}}_{q}^{\mathrm{Dr}} / \mathcal{I}_{\text {const }}^{+}(H X)$, so that $\mathcal{I}^{+}(R)=\mathcal{I}_{\text {const }}^{+}(R)$ thanks to Remark $9.2(\mathrm{i}) \&(\mathrm{ii})$.

It follows that $\mathcal{I}^{+}(R) \subseteq\left(\mathcal{I}^{+}(H X), \mathcal{I}_{\text {const }}^{+}(R)\right)$.

(iii) follows from (i) and (ii) thanks to Remarks 9.3 and 7.3.

Remark 9.16. In Proposition 9.15(i) the hypothesis $\tilde{l}=0$ is not necessary: the claim would hold also in case $\tilde{l}=1$. But this case is omitted here because it is not really needed in this paper and its proof, very similar, would just require a little more complicated, and repetitive, exposition (see the proof of Proposition $9.15($ ii) $)$.

Corollary 9.17. (i) $\mathcal{I}^{ \pm}(X 1)=\mathcal{I}_{\text {const }}^{ \pm}(X 1)$ and $\mathcal{I}^{ \pm}(X 2)=\mathcal{I}_{\text {const }}^{ \pm}(X 2)$.

(ii) If $\left(R^{ \pm}\right)$is one of $\left(X 3^{\varepsilon, \pm}\right)-\left(S 3^{ \pm}\right),\left(T k^{ \pm}\right)$and $\left(S^{ \pm}\right)$then

$$
\mathcal{I}^{ \pm}(R) \subseteq\left(\mathcal{I}^{ \pm}(H X), \mathcal{I}_{\text {const }}^{ \pm}(R)\right)
$$

(iii) If $\left(R^{ \pm}\right)$is one of $\left(X 3^{\varepsilon, \pm}\right)-\left(S 3^{ \pm}\right)$and $\left(S^{ \pm}\right)$then

$$
\mathcal{I}^{ \pm}(R, X D, X 1, X 2)=\mathcal{I}_{\text {const }}^{ \pm}(R, X D, X 1, X 2) .
$$


Proof. The claims follow from Proposition 9.15, Remarks 9.8 and 9.10 and Proposition 7.15 .

Remark 9.18. Remark 9.16 would imply that furthermore $\mathcal{I}^{ \pm}(R)=\mathcal{I}_{\text {const }}^{ \pm}(R)$ even in the case when $\left(R^{ \pm}\right)$is one of $\left(S k^{ \pm}\right),\left(T k^{ \pm}\right)$and, if $a_{i j}=-1$, also $\left(S(U L)_{(i, j)}^{ \pm}\right)$.

Corollary 9.19. Proposition 9.15 implies that

$$
\mathcal{U}_{q}^{\text {Dr }}=\tilde{\mathcal{U}}_{q}^{\text {Dr }} / \mathcal{I}_{\text {const }}\left(X D, X 1, X 2, X 3^{\varepsilon}, S U L, S 2, S 3\right) .
$$

The final remark of this section is a straightforward consequence of Remark 8.5.

Remark 9.20. (i) If $\left(R^{ \pm}\right)$is one of $\left(X D^{ \pm}\right)-\left(X 2^{ \pm}\right),\left(S U L^{ \pm}\right)-\left(S 3^{ \pm}\right),\left(T k^{ \pm}\right),\left(S^{ \pm}\right)$ then $\mathcal{I}^{+}(R)$ and $\mathcal{I}_{\text {const }}^{+}(R)$ are $\tilde{\Theta}$-stable.

(ii) $\mathcal{I}^{+}\left(X 3^{1}, X 3^{-1}\right)$ and $\mathcal{I}_{\text {const }}^{+}\left(X 3^{1}, X 3^{-1}\right)$ are the $\tilde{\Theta}$-stable ideals generated respectively by $\mathcal{I}^{+}\left(X 3^{1}\right)$ and $\mathcal{I}_{\text {const }}^{+}\left(X 3^{1}\right)$;

(iii) $\mathcal{I}_{\text {const }}^{+}\left(X 3^{1}, X 3^{-1}\right)$ is the $\tilde{\Theta}$-stable and $t_{i}$-stable ideal (for all $\left.i \in I_{0}\right)$ generated by $\mathcal{I}_{0}^{+}\left(X 3^{1}\right)$;

(iv) $\mathcal{I}\left(X 3^{1}, X 3^{-1}, X 2\right)=\mathcal{I}_{\text {const }}\left(X 3^{1}, X 3^{-1}, X 2\right)$ is the $\tilde{\Omega}$-stable, $\tilde{\Theta}$-stable and $\tilde{t}_{i^{-}}$ stable ideal (for all $\left.i \in I_{0}\right)$ generated by $\mathcal{I}_{0}^{+}\left(X 3^{1}, X 2\right)$.

\section{$\S 10$. More about redundant relations}

In this section we prove some dependences among the relations $\left(X D^{ \pm}\right)-\left(S 3^{ \pm}\right)$, making systematic recourse to the properties of $q$-commutators (Remark 4.17) and to Corollary 9.5(ii).

Proposition 10.1. With the notation of Remark 9.1,

$$
\mathcal{I}_{\text {const }}^{ \pm}(X 2), \mathcal{I}_{\text {const }}^{ \pm}\left(X 3^{-1}\right) \subseteq \mathcal{I}_{\text {const }}^{ \pm}\left(X 3^{1}\right) .
$$

Proof. To prove $\mathcal{I}_{0}^{+}(X 2) \subseteq \mathcal{I}_{\text {const }}^{+}\left(X 3^{1}\right)$, note that

$$
\left[\left[\left[X_{1,1}^{+}, X_{1,0}^{+}\right]_{q^{2}}, X_{1,0}^{+}\right]_{q^{4}}, X_{1,-1}^{-}\right] \in \mathcal{I}_{\text {const }}^{+}\left(X 3^{1}\right) ;
$$

but

$$
\begin{aligned}
& {\left[\left[\left[X_{1,1}^{+}, X_{1,0}^{+}\right]_{q^{2}}, X_{1,0}^{+}\right]_{q^{4}}, X_{1,-1}^{-}\right]=\left[\left[\frac{C k_{1}-C^{-1} k_{1}^{-1}}{q-q^{-1}}, X_{1,0}^{+}\right]_{q^{2}}, X_{1,0}^{+}\right]_{q^{4}}} \\
& +\left[\left[X_{1,1}^{+}, k_{1}^{-1} H_{1,-1}\right]_{q^{2}}, X_{1,0}^{+}\right]_{q^{4}}+\left[\left[X_{1,1}^{+}, X_{1,0}^{+}\right]_{q^{2}}, k_{1}^{-1} H_{1,-1}\right]_{q^{4}} \\
& =k_{1}^{-1}\left(q^{2}[2]_{q} C^{-1}\left[X_{1,0}^{+}, X_{1,0}^{+}\right]_{q^{6}}-q^{2}\left[\left[H_{1,-1}, X_{1,1}^{+}\right], X_{1,0}^{+}\right]_{q^{6}}-q^{4}\left[H_{1,-1},\left[X_{1,1}^{+}, X_{1,0}^{+}\right]_{q^{2}}\right]\right) \\
& =-q^{4}[3]_{q} ! C^{-1} k_{1}^{-1}\left(\left[X_{1,1}^{+}, X_{1,-1}^{+}\right]_{q^{2}}-\left(q^{4}-q^{-2}\right)\left(X_{1,0}^{+}\right)^{2}\right)=-q^{4}[3]_{q} ! C^{-1} k_{1}^{-1} M_{(2,2)}^{+}(0),
\end{aligned}
$$

so $\mathcal{I}_{0}^{+}(X 2) \subseteq \mathcal{I}_{\text {const }}^{+}\left(X 3^{1}\right)$. 
For the other inclusion, notice that

$$
\begin{aligned}
\mathcal{I}_{\text {const }}^{+} & \left(X 3^{1}\right) \ni \frac{1}{b_{i i \tilde{d}_{i}}} \tilde{t}_{1}\left(\left[H_{1,1}, M_{(3)}^{1,+}(0)\right]\right) \\
= & {\left[\left[X_{1,1}^{+}, X_{1,-1}^{+}\right]_{q^{2}}, X_{1,-1}^{+}\right]_{q^{4}}+\left[\left[X_{1,0}^{+}, X_{1,0}^{+}\right]_{q^{2}}, X_{1,-1}^{+}\right]_{q^{4}}+\left[\left[X_{1,0}^{+}, X_{1,-1}^{+}\right]_{q^{2}}, X_{1,0}^{+}\right]_{q^{4}} } \\
= & {\left[\left[X_{1,1}^{+}, X_{1,-1}^{+}\right]_{q^{2}}-\left(q^{4}-q^{-2}\right)\left(X_{1,0}^{+}\right)^{2}, X_{1,-1}^{+}\right]_{q^{4}} } \\
& +\left(q^{4}-q^{-2}+1-q^{2}-q^{4}\right)\left(X_{1,0}^{+}\right)^{2} X_{1,-1}^{+} \\
& +\left(1+q^{6}\right) X_{1,0}^{+} X_{1,-1}^{+} X_{1,0}^{+}+\left(-q^{8}+q^{2}-q^{4}+q^{6}-q^{2}\right) X_{1,-1}^{+}\left(X_{1,0}^{+}\right)^{2}
\end{aligned}
$$

so that

$$
\begin{gathered}
-\left(q^{2}-1+q^{-2}\right)\left(\left(X_{1,0}^{+}\right)^{2} X_{1,-1}^{+}-\left(q^{4}+q^{2}\right) X_{1,0}^{+} X_{1,-1}^{+} X_{1,0}^{+}+q^{6} X_{1,-1}^{+}\left(X_{1,0}^{+}\right)^{2}\right) \\
=-q^{6}\left(q^{2}-1+q^{-2}\right)\left[\left[X_{1,-1}^{+}, X_{1,0}^{+}\right]_{q^{-2}}, X_{1,0}^{+}\right]_{q^{-4}}=-q^{6}\left(q^{2}-1+q^{-2}\right) M_{(3)}^{-1,+}(0)
\end{gathered}
$$

is an element of $\mathcal{I}_{\text {const }}^{+}\left(X 3^{1}\right)$. The claim follows again from Corollary 9.5.

Corollary 10.2. (i) $\mathcal{I}^{ \pm}\left(X 3^{1}\right)=\mathcal{I}_{\text {const }}^{ \pm}\left(X 3^{1}\right)$ (see Corollary $9.17($ iii $\left.)\right)$;

(ii) $\mathcal{I}_{\text {const }}^{+}\left(X 3^{1}\right)=\mathcal{I}_{\text {const }}^{+}\left(X 3^{-1}\right)$ is $\tilde{\Theta}$-stable;

(iii) $\left(\mathcal{I}_{\text {const }}^{+}\left(X 3^{1}\right), \mathcal{I}_{\text {const }}^{-}\left(X 3^{-1}\right)\right)=\mathcal{I}_{\text {const }}\left(X 3^{1}\right)=\mathcal{I}_{\text {const }}\left(X 3^{-1}\right)$ is $\tilde{\Omega}$-stable.

Proposition 10.3. (i) $\mathcal{U}_{q}^{\operatorname{Dr}}\left(A_{1}^{(1)}\right)=\tilde{\mathcal{U}}_{q}^{\operatorname{Dr}}\left(A_{1}^{(1)}\right) / \mathcal{I}_{\text {const }}(X 1)$;

(ii) $\mathcal{U}_{q}^{\operatorname{Dr}}\left(A_{2}^{(2)}\right)=\tilde{\mathcal{U}}_{q}^{\operatorname{Dr}}\left(A_{2}^{(2)}\right) / \mathcal{I}_{\text {const }}\left(X 3^{1}\right)=\tilde{\mathcal{U}}_{q}^{\mathrm{Dr}}\left(A_{2}^{(2)}\right) /\left(\mathcal{I}_{\text {const }}^{+}\left(X 3^{1}\right), \mathcal{I}_{\text {const }}^{-}\left(X 3^{-1}\right)\right)$.

Proposition 10.4. $\mathcal{I}_{\text {const }}^{ \pm}(X D) \subseteq \mathcal{I}_{\text {const }}^{ \pm}(S U L)$.

Proof. Let $i, j \in I_{0}$ be such that $a_{i j}<0$; since $-1 \in\left\{a_{i j}, a_{j i}\right\}$, in the study of $\left[X_{i, \tilde{d}_{i j}}^{+}, X_{j, 0}^{+}\right]_{q_{i} a_{i j}}+\left[X_{j, \tilde{d}_{i j}}^{+}, X_{i, 0}^{+}\right]_{q_{i} a_{i j}}$ we can suppose that $a_{i j}=-1$, and in particular $\tilde{d}_{j} \leq \tilde{d}_{i}=\tilde{d}_{i j}$ and, if $X_{\tilde{n}}^{(k)}=A_{2 n}^{(2)}, i \neq 1$. Then $\left[\left[X_{j, 0}^{+}, X_{i, 0}^{+}\right]_{q}, X_{i, 0}^{+}\right]_{q^{-1}}$ is an element of $\mathcal{I}_{\text {const }}^{+}(S U L)$, and so is $\left[\left[\left[X_{j, 0}^{+}, X_{i, 0}^{+}\right]_{q}, X_{i, 0}^{+}\right]_{q^{-1}}, X_{i, \tilde{d}_{i}}^{-}\right]$. But

$$
\begin{aligned}
& {\left[\left[\left[X_{j, 0}^{+}, X_{i, 0}^{+}\right]_{q}, X_{i, 0}^{+}\right]_{q^{-1}}, X_{i, \tilde{d}_{i}}^{-}\right]} \\
& \quad=\left[\left[X_{j, 0}^{+}, X_{i, 0}^{+}\right]_{q}, C^{-\tilde{d}_{i}} k_{i} H_{i, \tilde{d}_{i}}\right]_{q^{-1}}+\left[\left[X_{j, 0}^{+}, C^{-\tilde{d}_{i}} k_{i} H_{i, \tilde{d}_{i}}\right]_{q}, X_{i, 0}^{+}\right]_{q^{-1}} \\
& \quad=C^{-\tilde{d}_{i}} k_{i}\left(q^{-1}\left[\left[X_{j, 0}^{+}, X_{i, 0}^{+}\right]_{q}, H_{i, \tilde{d}_{i}}\right]+q\left[\left[X_{j, 0}^{+}, H_{i, \tilde{d}_{i}}\right], X_{i, 0}^{+}\right]_{q^{-3}}\right) \\
& \quad=C^{-\tilde{d}_{i}} k_{i}\left(-b_{i i \tilde{d}_{i}} q^{-1}\left[X_{j, 0}^{+}, X_{i, \tilde{d}_{i}}^{+}\right]_{q}-b_{i j \tilde{d}_{i}} q^{-1}\left[X_{j, \tilde{d}_{i}}^{+}, X_{i, 0}^{+}\right]_{q}-b_{i j \tilde{d}_{i}} q\left[X_{j, \tilde{d}_{i}}^{+}, X_{i, 0}^{+}\right]_{q^{-3}}\right) \\
& \quad=[2]_{q_{i}} C^{-\tilde{d}_{i}} k_{i}\left(\left[X_{i, \tilde{d}_{i}}^{+}, X_{j, 0}^{+}\right]_{q^{-1}}+\left[X_{j, \tilde{d}_{i}}^{+}, X_{i, 0}^{+}\right]_{q^{-1}}\right) .
\end{aligned}
$$

Thus $\mathcal{I}_{0}^{+}(X D) \subseteq \mathcal{I}_{\text {const }}^{+}(S U L)$, and the claim follows using Corollary 9.5.

Lemma 10.5. For $i \in I_{0}, a \in \mathbb{N}$ define $Y_{i, a} \in \tilde{\mathcal{U}}_{q}^{\mathrm{Dr}}\left(X_{\tilde{n}}^{(k)}\right)$ as follows:

$$
Y_{i, 0}=X_{i, \tilde{d}_{i}}^{+}, \quad Y_{i, a+1}=\left[Y_{i, a}, X_{i, 0}^{+}\right]_{q_{i}^{2(a+1)}} .
$$


(Notice that $Y_{i, 1}=M_{i}^{+}(0)$.) Then:

(i) $\left[Y_{i, a}, X_{i, 0}^{-}\right]=\left(b_{i i \tilde{d}_{i}}-[a]_{q_{i}}[a+1]_{q_{i}}\right) k_{i} Y_{i, a-1}$ for all $a>0$;

(ii) $\left[X_{i, j ; 1-a_{i j} ; 1}^{+}(0 ; 0), X_{j, \tilde{d}_{i j}}^{-}\right]=C^{-\tilde{d}_{i j}} k_{j} b_{j i \tilde{d}_{i j}} Y_{i,-a_{i j}}$.

Proof. (i) We have

$$
\begin{aligned}
& {\left[Y_{i, a}, X_{i, 0}^{-}\right]=\left[\left[\ldots\left[\ldots\left[X_{i, \tilde{d}_{i}}^{+}, X_{i, 0}^{+}\right]_{q_{i}^{2}}, \ldots X_{i, 0}^{+}\right]_{q_{i}^{2 u}}, \ldots X_{i, 0}^{+}\right]_{q_{i}^{2 a}}, X_{i, 0}^{-}\right]} \\
& =\left[\ldots\left[\ldots\left[k_{i} H_{i, \tilde{d}_{i}}, X_{i, 0}^{+}\right]_{q_{i}^{2}}, \ldots X_{i, 0}^{+}\right]_{q_{i}^{2 u}}, \ldots X_{i, 0}^{+}\right]_{q_{i}^{2 a}} \\
& +\sum_{u=1}^{a}\left[\ldots\left[\left[\left[\ldots\left[X_{i, \tilde{d}_{i}}^{+}, X_{i, 0}^{+}\right]_{q_{i}^{2}}, \ldots X_{i, 0}^{+}\right]_{q_{i}^{2(u-1)}}, \frac{k_{i}-k_{i}^{-1}}{q_{i}-q_{i}^{-1}}\right]_{q_{i}^{2 u}}, X_{i, 0}^{+}\right]_{q_{i}^{2(u+1)}}, \ldots X_{i, 0}^{+}\right]_{q_{i}^{2 a}} \\
& =k_{i}\left[\ldots\left[\ldots\left[H_{i, \tilde{d}_{i}}, X_{i, 0}^{+}\right], \ldots X_{i, 0}^{+}\right]_{q_{i}^{2(u-1)}}, \ldots X_{i, 0}^{+}\right]_{q_{i}^{2(a-1)}} \\
& \quad+\sum_{u=1}^{a} \frac{q_{i}^{-2 u}-q_{i}^{2 u}}{q_{i}-q_{i}^{-1}} k_{i}\left[\ldots\left[\left[\ldots\left[X_{i, \tilde{d}_{i}}^{+}, X_{i, 0}^{+}\right]_{q_{i}^{2}}, \ldots X_{i, 0}^{+}\right]_{q_{i}^{2(u-1)}}, X_{i, 0}^{+}\right]_{q_{i}^{2 u}}, \ldots X_{i, 0}^{+}\right]_{q_{i}^{2(a-1)}} \\
& =b_{i i \tilde{d}_{i}} k_{i}\left[\ldots\left[\ldots\left[X_{i, \tilde{d}_{i}}^{+}, X_{i, 0}^{+}\right]_{q_{i}^{2}}, \ldots X_{i, 0}^{+}\right]_{q_{i}^{2(u-1)}}, \ldots X_{i, 0}^{+}\right]_{q_{i}^{2(a-1)}} \\
& \quad-\sum_{u=1}^{a}[2 u]_{q_{i}} k_{i}\left[\ldots\left[\left[\ldots\left[X_{i, \tilde{d}_{i}}^{+}, X_{i, 0}^{+}\right]_{q_{i}^{2}}, \ldots X_{i, 0}^{+}\right]_{q_{i}^{2(u-1)}}, X_{i, 0}^{+}\right]_{q_{i}^{2 u}}, \ldots X_{i, 0}^{+}\right]_{q_{i}^{2(a-1)}} \\
& =\left(b_{i i \tilde{d}_{i}}-[a]_{q_{i}}[a+1]_{q_{i}}\right) k_{i} Y_{i, a-1} .
\end{aligned}
$$

(ii) We have

$$
\begin{aligned}
& {\left[X_{i, j ; 1-a_{i j} ; 1}^{+}(0 ; 0), X_{j, \tilde{d}_{i j}}^{-}\right]} \\
& =\left[\left[\ldots\left[\ldots\left[X_{j, 0}^{+}, X_{i, 0}^{+}\right]_{q_{i} a_{i j}}, \ldots X_{i, 0}^{+}\right]_{q_{i} a_{i j}+2(u-1)}, \ldots X_{i, 0}^{+}\right]_{q_{i}{ }^{-a_{i j}}}, X_{j, \tilde{d}_{i j}}^{-}\right] \\
& =\frac{1}{q_{j}-q_{j}^{-1}}\left[\ldots\left[\ldots\left[C^{-\tilde{d}_{i j}} k_{j} \tilde{H}_{j, \tilde{d}_{i j}}^{+}, X_{i, 0}^{+}\right]_{q_{i} a_{i j}}, \ldots X_{i, 0}^{+}\right]_{q_{i}^{a_{i j}+2(u-1)}}, \ldots X_{i, 0}^{+}\right]_{q_{i}{ }^{-a_{i j}}} \\
& =\frac{C^{-\tilde{d}_{i j}} k_{j}}{q_{j}-q_{j}^{-1}}\left[\ldots\left[\ldots\left[\tilde{H}_{j, \tilde{d}_{i j}}^{+}, X_{i, 0}^{+}\right], \ldots X_{i, 0}^{+}\right]_{q_{i}^{2(u-1)}}, \ldots X_{i, 0}^{+}\right]_{q_{i}^{-2 a_{i j} j}} .
\end{aligned}
$$

Recalling Remark 7.18 we get

$$
\begin{aligned}
& {\left[X_{i, j ; 1-a_{i j} ; 1}^{+}(0 ; 0), X_{j, \tilde{d}_{i j}}^{-}\right]} \\
& \quad=C^{-\tilde{d}_{i j}} k_{j} b_{j i \tilde{d}_{i j}}\left[\ldots\left[\ldots\left[X_{i, \tilde{d}_{i j}}^{+}, X_{i, 0}^{+}\right]_{q_{i}^{2}}, \ldots X_{i, 0}^{+}\right]_{q_{i}^{2(u-1)}}, \ldots X_{i, 0}^{+}\right]_{q_{i}^{-2 a_{i j}}} \\
& \quad=C^{-\tilde{d}_{i j}} k_{j} b_{j i \tilde{d}_{i j}} Y_{i,-a_{i j}} .
\end{aligned}
$$

Corollary 10.6. Let $i, j \in I_{0}$ be such that $a_{i j}<0$ with the condition that $a_{i j}=-1$ if $k>1$; then $M_{i}^{+}(0) \in \mathcal{I}_{\text {const }}^{+}(S U L)$. In particular: 
(i) in the cases of rank greater than 1 (that is, $X_{\tilde{n}}^{(k)} \neq A_{1}^{(1)}, A_{2}^{(2)}$ ) and different from $D_{n+1}^{(2)}$ and $D_{4}^{(3)}$ we have $\mathcal{I}_{\text {const }}^{ \pm}(X 1) \subseteq \mathcal{I}_{\text {const }}^{ \pm}(S U L)$;

(ii) in cases $D_{n+1}^{(2)}$ and $D_{4}^{(3)}$ we have $\mathcal{I}_{\text {const }}^{ \pm}(X 1) \subseteq\left(\mathcal{I}_{\text {const }}^{ \pm}(S U L), \mathcal{I}_{\text {const }}^{ \pm}\left(X 1_{1}\right)\right)$.

Proof. This is an immediate consequence of Lemma 10.5 (and of Corollary 9.5) once one notices that the hypotheses imply that $X_{i, j ; 1-a_{i j} ; 1}^{+}(0 ; 0) \in \mathcal{I}_{\text {const }}^{+}(S U L)$, $b_{j i \tilde{d}_{i j}} \neq 0$ and $b_{i i \tilde{d}_{i}}=[2]_{q_{i}}$.

Remark 10.7. If $k=2, X_{\tilde{n}}^{(k)} \neq A_{2 n}^{(2)}$ and $i, j \in I_{0}$ are such that $a_{i j}=-2$ then

$$
\mathcal{I}_{\text {const }}^{ \pm}\left(X 1_{i}\right) \subseteq \mathcal{I}_{\text {const }}^{ \pm}(T 2) \text {. }
$$

In particular $\mathcal{I}_{\text {const }}^{ \pm}(T 2)=\mathcal{I}_{\text {const }}^{ \pm}\left(X 1_{i}, S 2\right)$.

Proof. $\left[\left[X_{j, 0}^{+}, X_{i, 1}^{+}\right]_{q^{2}}, X_{i, 0}^{+}\right]$lies in $\mathcal{I}_{\text {const }}^{+}(T 2)$ and so does

$$
\left[\left[\left[X_{j, 0}^{+}, X_{i, 1}^{+}\right]_{q^{2}}, X_{i, 0}^{+}\right], X_{j, 0}^{-}\right]=\left[\left[\frac{k_{j}-k_{j}^{-1}}{q^{2}-q^{-2}}, X_{i, 1}^{+}\right]_{q^{2}}, X_{i, 0}^{+}\right]=-q^{2} k_{j}\left[X_{i, 1}^{+}, X_{i, 0}^{+}\right]_{q^{2}} .
$$

Theorem 10.8.

(i) $\mathcal{U}_{q}^{\operatorname{Dr}}\left(X_{\tilde{n}}^{(1)}\right)= \begin{cases}\tilde{\mathcal{U}}_{q}^{\operatorname{Dr}}\left(A_{1}^{(1)}\right) / \mathcal{I}_{\text {const }}(X 1) & \text { if } X_{\tilde{n}}=A_{1}, \\ \tilde{\mathcal{U}}_{q}^{\operatorname{Dr}}\left(X_{\tilde{n}}^{(1)}\right) / \mathcal{I}_{\text {const }}(S U L) & \text { otherwise; }\end{cases}$

(ii) $\mathcal{U}_{q}^{\operatorname{Dr}}\left(X_{\tilde{n}}^{(2)}\right)= \begin{cases}\tilde{\mathcal{U}}_{q}^{\operatorname{Dr}}\left(A_{2 n}^{(2)}\right) / \mathcal{I}_{\text {const }}\left(X 3^{1}, S U L, S 2\right) & \text { if } X_{\tilde{n}}=A_{2 n}, \\ \tilde{\mathcal{U}}_{q}^{\operatorname{Dr}}\left(X_{\tilde{n}}^{(2)}\right) / \mathcal{I}_{\text {const }}(S U L, T 2) & \text { otherwise; }\end{cases}$

(iii) $\mathcal{U}_{q}^{\mathrm{Dr}}\left(D_{4}^{(3)}\right)=\tilde{\mathcal{U}}_{q}^{\mathrm{Dr}}\left(D_{4}^{(3)}\right) / \mathcal{I}_{\text {const }}\left(X 1_{1}, S U L, S 3\right)$

$$
=\tilde{\mathcal{U}}_{q}^{\mathrm{Dr}}\left(D_{4}^{(3)}\right) / \mathcal{I}_{\text {const }}\left(X 1_{1}, S U L, T 3\right) .
$$

Corollary 10.9. Let $U$ be a $\mathbb{C}(q)$-algebra, $t_{i}^{(U)}\left(i \in I_{0}\right)$ be $\mathbb{C}(q)$-automorphisms of $U, \Omega^{(U)}$ be a $\mathbb{C}$-antilinear antiautomorphism of $U$, and $\tilde{f}: \tilde{\mathcal{U}}_{q}^{\mathrm{Dr}}\left(X_{\tilde{n}}^{(k)}\right) \rightarrow U$ be a homomorphism of $\mathbb{C}(q)$-algebras such that $\tilde{f} \circ \tilde{t}_{i}=t_{i}^{(U)} \circ \tilde{f}$ for all $i \in I_{0}$ and $\tilde{f} \circ \tilde{\Omega}=\Omega^{(U)} \circ \tilde{f} . I f:$

(i) $\tilde{f}\left(\mathcal{I}_{0}^{+}(X 1)\right)=0$ in case $X_{\tilde{n}}^{(k)}=A_{1}^{(1)}$;

(ii) $\tilde{f}\left(\mathcal{I}_{0}^{+}(S U L)\right)=0$ in case $k=1, X_{\tilde{n}}^{(k)} \neq A_{1}^{(1)}$;

(iii) $\tilde{f}\left(\mathcal{I}_{0}^{+}\left(X 3^{1}, S U L, S 2\right)\right)=0$ in case $X_{\tilde{n}}^{(k)}=A_{2 n}^{(2)}$;

(iv) $\tilde{f}\left(\mathcal{I}_{0}^{+}(S U L, T 2)\right)=0$ in case $k=2, X_{\tilde{n}}^{(k)} \neq A_{2 n}^{(2)}$;

(v) $\tilde{f}\left(\mathcal{I}_{0}^{+}\left(X 1_{1}, S U L, T 3\right)\right)=0$ in case $D_{4}^{(3)}$,

then $\tilde{f}$ induces $f: \mathcal{U}_{q}^{\operatorname{Dr}}\left(X_{\tilde{n}}^{(k)}\right) \rightarrow U$ and we have $f \circ t_{i}=t_{i}^{(U)} \circ f$ for all $i \in I_{0}$, and $f \circ \Omega=\Omega^{(U)} \circ f$. 


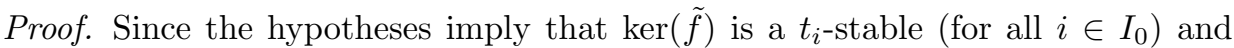
$\tilde{\Omega}$-stable ideal of $\mathcal{U}_{q}^{\mathrm{Dr}}\left(X_{\tilde{n}}^{(k)}\right)$, the claim is an immediate consequence of Theorem 10.9 and of Corollary 9.5(i).

\section{$\S 11$. The Serre relations}

This section is devoted to the study of the Serre relations (see Definition 5.9). In particular we prove that the Serre relations hold in $\mathcal{U}_{q}^{\mathrm{Dr}}$, and that in the case of rank greater than 1 the Serre relations alone are indeed equivalent to $\left(X D^{ \pm}\right)-\left(S 3^{ \pm}\right)$(in $\left.\tilde{\mathcal{U}}_{q}^{\mathrm{Dr}}\right)$, that is, $\mathcal{U}_{q}^{\mathrm{Dr}}=\tilde{\mathcal{U}}_{q}^{\mathrm{Dr}} / \mathcal{I}_{\text {const }}\left(S^{ \pm}\right)$. We use the notation fixed in Notations 5.1 and 9.1.

Remark 11.1. (i) If $k=1\left(S^{ \pm}\right)=\left(S U L^{ \pm}\right)$;

(ii) if $k>1$ and $i, j \in I_{0}$ are such that $a_{i j}<-1$ then $\left(S^{ \pm}\right)=\left(S U L^{ \pm}\right) \cup\left(S_{(i, j)}^{ \pm}\right)$.

Before passing to the proof that the Serre relations hold in $\mathcal{U}_{q}^{\mathrm{Dr}}$, we state the following remark on $q$-commutators, which simplifies many computations in the next propositions.

Lemma 11.2. Let $a \in \tilde{\mathcal{U}}_{q}^{\mathrm{Dr}}$ and $i \in I_{0}$ be such that $\left(X_{\tilde{n}}^{(k)}, i\right) \neq\left(A_{2 n}^{(2)}, 1\right)$, and let $u, v \in \mathbb{C}(q)$. Then in $\tilde{\mathcal{U}}_{q}^{\mathrm{Dr}} / \mathcal{I}_{\text {const }}^{+}\left(X 1_{i}\right)$ we have, for all $r \in \mathbb{Z}$ :

(i) $\left[\left[a, X_{i, r}^{+}\right]_{u}, X_{i, r+\tilde{d}_{i}}^{+}\right]_{v}=q_{i}^{-2}\left[\left[a, X_{i, r+\tilde{d}_{i}}^{+}\right]_{q_{i}^{2} v}, X_{i, r}^{+}\right]_{q_{i}^{2} u}$;

(ii) $\left[\left[a, X_{i, r+2 \tilde{d}_{i}}^{+}\right]_{u}, X_{i, r}^{+}\right]_{v}=q_{i}^{2}\left[\left[a, X_{i, r}^{+}\right]_{q_{i}^{-2} v}, X_{i, r+2 \tilde{d}_{i}}^{+}\right]_{q_{i}^{-2} u}+\left(q^{2}-1\right)\left[a,\left(X_{i, r+\tilde{d}_{i}}^{+}\right)^{2}\right]_{q_{i}^{-2} u v}$.

Proof. It is a simple computation using Remark 4.17(iii).

Proposition 11.3. If $k=2, X_{\tilde{n}}^{(2)} \neq A_{2 n}^{(2)}$ and $i, j \in I_{0}$ are such that $a_{i j}=-2$, then

$$
\mathcal{I}_{\text {const }}^{ \pm}\left(S_{(i, j)}\right) \subseteq \mathcal{I}_{\text {const }}^{ \pm}(T 2)
$$

Proof. In this proof we use that $\mathcal{I}_{\text {const }}^{ \pm}\left(X 1_{i}\right) \subseteq \mathcal{I}_{\text {const }}^{ \pm}(T 2)$ (see Proposition 10.7) and make computations in $\tilde{\mathcal{U}}_{q}^{\mathrm{Dr}} / \mathcal{I}_{\text {const }}^{+}(T 2)$.

Since $\left[H_{i, 1},\left[\left[X_{j, 0}^{+}, X_{i, 0}^{+}\right]_{q^{2}}, X_{i,-1}^{+}\right]\right]$lies in $\mathcal{I}_{\text {const }}^{+}(T 2)$ (see Definition 5.7) we see that

$$
\left[\left[X_{j, 0}^{+}, X_{i, 1}^{+}\right]_{q^{2}}, X_{i,-1}^{+}\right]+\left[\left[X_{j, 0}^{+}, X_{i, 0}^{+}\right]_{q^{2}}, X_{i, 0}^{+}\right]=0
$$

in $\tilde{\mathcal{U}}_{q}^{\mathrm{Dr}} / \mathcal{I}_{\text {const }}^{+}(T 2)$; but, thanks to Lemma 11.2(ii), we have

$$
\left[\left[X_{j, 0}^{+}, X_{i, 1}^{+}\right]_{q^{2}}, X_{i,-1}^{+}\right]=q^{2}\left[\left[X_{j, 0}^{+}, X_{i,-1}^{+}\right]_{q^{-2}}, X_{i, 1}^{+}\right]+\left(q^{2}-1\right)\left[X_{j, 0}^{+},\left(X_{i, 0}^{+}\right)^{2}\right],
$$


so that

$$
\begin{aligned}
0 & =q^{2}\left[\left[X_{j, 0}^{+}, X_{i,-1}^{+}\right]_{q^{-2}}, X_{i, 1}^{+}\right]+\left[X_{j, 0}^{+},\left(q^{2}-1\right)\left(X_{i, 0}^{+}\right)^{2}\right]+\left[\left[X_{j, 0}^{+}, X_{i, 0}^{+}\right]_{q^{2}}, X_{i, 0}^{+}\right] \\
& =q^{2}\left[\left[X_{j, 0}^{+}, X_{i,-1}^{+}\right]_{q^{-2}}, X_{i, 1}^{+}\right]+q^{2}\left[\left[X_{j, 0}^{+}, X_{i, 0}^{+}\right]_{q^{-2}}, X_{i, 0}^{+}\right]
\end{aligned}
$$

and also

$$
\left[\left[\left[X_{j, 0}^{+}, X_{i,-1}^{+}\right]_{q^{-2}}, X_{i, 1}^{+}\right]+\left[\left[X_{j, 0}^{+}, X_{i, 0}^{+}\right]_{q^{-2}}, X_{i, 0}^{+}\right], X_{i, 0}^{+}\right]_{q^{2}}=0 .
$$

Now, thanks to Lemma $11.2(\mathrm{i})$ and to the relations $\left(T 2^{+}\right)$,

$$
\left[\left[\left[X_{j, 0}^{+}, X_{i,-1}^{+}\right]_{q^{-2}}, X_{i, 1}^{+}\right], X_{i, 0}^{+}\right]_{q^{2}}=\left[\left[\left[X_{j, 0}^{+}, X_{i, 0}^{+}\right]_{q^{2}}, X_{i,-1}^{+}\right], X_{i, 1}^{+}\right]_{q^{-2}}=0,
$$

so that

$$
\left[\left[\left[X_{j, 0}^{+}, X_{i, 0}^{+}\right]_{q^{-2}}, X_{i, 0}^{+}\right], X_{i, 0}^{+}\right]_{q^{2}}=0,
$$

which implies $\mathcal{I}_{\text {const }}^{ \pm}\left(S_{(i, j)}\right) \subseteq \mathcal{I}_{\text {const }}^{ \pm}(T 2)$, thanks to Corollary 9.5 .

Let us concentrate now on case $A_{2 n}^{(2)}$.

Lemma 11.4. Let $X_{\tilde{n}}^{(k)}=A_{2 n}^{(2)}$; then

$$
\left[\left[X_{1,2}^{+}, X_{1,1}^{+}\right]_{q^{2}}, X_{1,0}^{+}\right]_{q^{4}}-\left(q^{2}-1\right)\left(q^{4}-1\right)\left(q^{2}+q^{-2}\right)\left(X_{1,1}^{+}\right)^{3} \in \mathcal{I}_{\text {const }}^{+}\left(X 3^{1}\right) .
$$

Proof. By Corollary 10.2,

$$
\left.\left[\left[X_{1,2}^{+}, X_{1,1}^{+}\right]_{q^{2}}, X_{1,0}^{+}\right]_{q^{4}}+\left[\left[X_{1,2}^{+}, X_{1,0}^{+}\right]_{q^{2}}, X_{1,1}^{+}\right]_{q^{4}}+\left[X_{1,1}^{+}, X_{1,1}^{+}\right]_{q^{2}}, X_{1,1}^{+}\right]_{q^{4}}
$$

belongs to $\mathcal{I}^{+}\left(X 3^{1}\right)=\mathcal{I}_{\text {const }}^{+}\left(X 3^{1}\right)$.

But $\left[X_{1,2}^{+}, X_{1,0}^{+}\right]_{q^{2}}-\left(q^{4}-q^{-2}\right)\left(X_{1,1}^{+}\right)^{2} \in \mathcal{I}_{\text {const }}^{+}\left(X 3^{1}\right)$ (see Proposition 10.1), so that

$$
\left[\left[X_{1,2}^{+}, X_{1,1}^{+}\right]_{q^{2}}, X_{1,0}^{+}\right]_{q^{4}}+\left(1-q^{4}\right)\left(q^{4}-q^{-2}+1-q^{2}\right)\left(X_{1,1}^{+}\right)^{3}
$$

lies in $\mathcal{I}_{\text {const }}^{+}\left(X 3^{1}\right)$.

Proposition 11.5. If $X_{\tilde{n}}^{(2)}=A_{2 n}^{(2)}$ and $i, j \in I_{0}$ are such that $a_{i j}=-2(i=1$, $j=2)$, then $\mathcal{I}_{\text {const }}^{ \pm}\left(S_{(i, j)}\right) \subseteq \mathcal{I}_{\text {const }}^{ \pm}\left(X D, X 3^{1}, S 2\right)$.

Proof. Recall that by the very definition of $\mathcal{I}_{\text {const }}^{+}(S 2)$ we have (see Remark 5.6)

$$
\left(q^{2}+q^{-2}\right)\left[\left[X_{j, 0}^{+}, X_{i, 1}^{+}\right]_{q^{2}}, X_{i, 0}^{+}\right]+q^{2}\left[\left[X_{i, 1}^{+}, X_{i, 0}^{+}\right]_{q^{2}}, X_{j, 0}^{+}\right]_{q^{-4}} \in \mathcal{I}_{\text {const }}^{+}(S 2),
$$

so that also

$$
\left(q^{2}+q^{-2}\right)\left[X_{i,-1}^{+},\left[\left[X_{j, 0}^{+}, X_{i, 1}^{+}\right]_{q^{2}}, X_{i, 0}^{+}\right]\right]_{q^{-2}}+q^{2}\left[X_{i,-1}^{+},\left[\left[X_{i, 1}^{+}, X_{i, 0}^{+}\right]_{q^{2}}, X_{j, 0}^{+}\right]_{q^{-4}}\right]_{q^{-2}}
$$

belongs to $\mathcal{I}_{\text {const }}^{+}(S 2)$, and let us compute the two summands separately in the algebra $\tilde{\mathcal{U}}_{q}^{\text {Dr }} / \mathcal{I}_{\text {const }}^{+}\left(X D, X 3^{1}, S 2\right)$ : 


$$
\begin{aligned}
& {\left[X_{i,-1}^{+},\left[\left[X_{j, 0}^{+}, X_{i, 1}^{+}\right]_{q^{2}}, X_{i, 0}^{+}\right]\right]_{q^{-2}}} \\
& =-q^{2}\left[X_{i,-1}^{+},\left[\left[X_{i, 1}^{+}, X_{j, 0}^{+}\right]_{q^{-2}}, X_{i, 0}^{+}\right]\right]_{q^{-2}} \stackrel{X D}{=} q^{2}\left[X_{i,-1}^{+},\left[\left[X_{j, 1}^{+}, X_{i, 0}^{+}\right]_{q^{-2}}, X_{i, 0}^{+}\right]\right]_{q^{-2}} \\
& =q^{2}\left[\left[X_{i,-1}^{+},\left[X_{j, 1}^{+}, X_{i, 0}^{+}\right]_{q^{-2}}\right], X_{i, 0}^{+}\right]_{q^{-2}}+q^{2}\left[\left[X_{j, 1}^{+}, X_{i, 0}^{+}\right]_{q^{-2}},\left[X_{i,-1}^{+}, X_{i, 0}^{+}\right]_{q^{-2}}\right] \\
& \stackrel{S 2}{=}-q^{4}\left[\left[X_{i, 0}^{+},\left[X_{j, 1}^{+}, X_{i,-1}^{+}\right]_{q^{-2}}\right]_{q^{-4}}, X_{i, 0}^{+}\right]_{q^{-2}}-\left[\left[X_{j, 1}^{+}, X_{i, 0}^{+}\right]_{q^{-2}},\left[X_{i, 0}^{+}, X_{i,-1}^{+}\right]_{q^{2}}\right] \\
& =-q^{4}\left[\left[X_{i, 0}^{+},\left[X_{j, 1}^{+}, X_{i,-1}^{+}\right]_{q^{-2}}\right]_{q^{-4}}, X_{i, 0}^{+}\right]_{q^{-2}} \\
& -\left[X_{j, 1}^{+},\left[X_{i, 0}^{+},\left[X_{i, 0}^{+}, X_{i,-1}^{+}\right]_{q^{2}}\right]_{q^{4}}\right]_{q^{-6}}+q^{-2}\left[X_{i, 0}^{+},\left[X_{j, 1}^{+},\left[X_{i, 0}^{+}, X_{i,-1}^{+}\right]_{q^{2}}\right]_{q^{-4}}\right]_{q^{6}} \\
& X \stackrel{D, X 3^{-1}}{=} q^{4}\left[\left[X_{i, 0}^{+},\left[X_{i, 0}^{+}, X_{j, 0}^{+}\right]_{q^{-2}}\right]_{q^{-4}}, X_{i, 0}^{+}\right]_{q^{-2}} \\
& +q^{-2}\left[X_{i, 0}^{+},\left[X_{j, 1}^{+},\left[X_{i, 0}^{+}, X_{i,-1}^{+}\right]_{q^{2}}\right]_{q^{-4}}\right]_{q^{6}} \\
& \stackrel{S 2}{=} q^{-2}\left[\left[\left[X_{j, 0}^{+}, X_{i, 0}^{+}\right]_{q^{2}}, X_{i, 0}^{+}\right]_{q^{4}}, X_{i, 0}^{+}\right]_{q^{-2}} \\
& -\left(1+q^{-4}\right)\left[X_{i, 0}^{+},\left[\left[X_{j, 1}^{+}, X_{i,-1}^{+}\right]_{q^{-2}}, X_{i, 0}^{+}\right]\right]_{q^{6}} \\
& \stackrel{X D}{=} q^{-2}\left[\left[\left[X_{j, 0}^{+}, X_{i, 0}^{+}\right]_{q^{2}}, X_{i, 0}^{+}\right]_{q^{4}}, X_{i, 0}^{+}\right]_{q^{-2}}+\left(q^{4}+1\right)\left[\left[\left[X_{j, 0}^{+}, X_{i, 0}^{+}\right]_{q^{2}}, X_{i, 0}^{+}\right], X_{i, 0}^{+}\right]_{q^{-6}} \\
& \text { and } \\
& {\left[X_{i,-1}^{+},\left[\left[X_{i, 1}^{+}, X_{i, 0}^{+}\right]_{q^{2}}, X_{j, 0}^{+}\right]_{q^{-4}}\right]_{q^{-2}}} \\
& =\left[\left[X_{i,-1}^{+},\left[X_{i, 1}^{+}, X_{i, 0}^{+}\right]_{q^{2}}\right]_{q^{-4}}, X_{j, 0}^{+}\right]_{q^{-2}}+q^{-4}\left[\left[X_{i, 1}^{+}, X_{i, 0}^{+}\right]_{q^{2}},\left[X_{i,-1}^{+}, X_{j, 0}^{+}\right]_{q^{2}}\right] \\
& \stackrel{X D}{=} q^{-6}\left[X_{j, 0}^{+},\left[\left[X_{i, 1}^{+}, X_{i, 0}^{+}\right]_{q^{2}}, X_{i,-1}^{+}\right]_{q^{4}}\right]_{q^{2}}+q^{-2}\left[\left[X_{i, 1}^{+}, X_{i, 0}^{+}\right]_{q^{2}},\left[X_{i, 0}^{+}, X_{j,-1}^{+}\right]_{q^{-2}}\right] \\
& \stackrel{X 3}{=}^{1}\left(1-q^{-2}\right)\left(1-q^{-4}\right)\left(q^{2}+q^{-2}\right)\left[X_{j, 0}^{+},\left(X_{i, 0}^{+}\right)^{3}\right]_{q^{2}} \\
& -q^{-2}\left[\left[X_{i, 0}^{+}, X_{j,-1}^{+}\right]_{q^{-2}},\left[X_{i, 1}^{+}, X_{i, 0}^{+}\right]_{q^{2}}\right] \\
& =\left(1-q^{-2}\right)\left(1-q^{-4}\right)\left(q^{2}+q^{-2}\right)\left[X_{j, 0}^{+},\left(X_{i, 0}^{+}\right)^{3}\right]_{q^{2}} \\
& -q^{-2}\left[X_{i, 0}^{+},\left[X_{j,-1}^{+},\left[X_{i, 1}^{+}, X_{i, 0}^{+}\right]_{q^{2}}\right]_{q^{4}}\right]_{q^{-6}}+q^{-4}\left[X_{j,-1}^{+},\left[X_{i, 0}^{+},\left[X_{i, 1}^{+}, X_{i, 0}^{+}\right]_{q^{2}}\right]_{q^{-4}}\right]_{q^{6}} \\
& {\stackrel{X 3^{1}}{=}}^{1}\left(1-q^{-2}\right)\left(1-q^{-4}\right)\left(q^{2}+q^{-2}\right)\left[X_{j, 0}^{+},\left(X_{i, 0}^{+}\right)^{3}\right]_{q^{2}} \\
& +q^{2}\left[X_{i, 0}^{+},\left[\left[X_{i, 1}^{+}, X_{i, 0}^{+}\right]_{q^{2}}, X_{j,-1}^{+}\right]_{q^{-4}}\right]_{q^{-6}} \\
& \stackrel{S 2}{=}\left(q^{2}+q^{-2}\right)\left(\left(1-q^{-2}\right)\left(1-q^{-4}\right)\left[X_{j, 0}^{+},\left(X_{i, 0}^{+}\right)^{3}\right]_{q^{2}}\right. \\
& \left.-\left[X_{i, 0}^{+},\left[\left[X_{j,-1}^{+}, X_{i, 1}^{+}\right]_{q^{2}}, X_{i, 0}^{+}\right]\right]_{q^{-6}}\right) \\
& \stackrel{X D}{=}\left(q^{2}+q^{-2}\right)\left(\left(1-q^{-2}\right)\left(1-q^{-4}\right)\left[X_{j, 0}^{+},\left(X_{i, 0}^{+}\right)^{3}\right]_{q^{2}}\right. \\
& \left.+q^{-4}\left[\left[\left[X_{j, 0}^{+}, X_{i, 0}^{+}\right]_{q^{-2}}, X_{i, 0}^{+}\right], X_{i, 0}^{+}\right]_{q^{6}}\right) .
\end{aligned}
$$

It follows that

$$
\begin{aligned}
q^{-2}\left[\left[\left[X_{j, 0}^{+},\right.\right.\right. & \left.\left.\left.X_{i, 0}^{+}\right]_{q^{2}}, X_{i, 0}^{+}\right]_{q^{4}}, X_{i, 0}^{+}\right]_{q^{-2}}+\left(q^{4}+1\right)\left[\left[\left[X_{j, 0}^{+}, X_{i, 0}^{+}\right]_{q^{2}}, X_{i, 0}^{+}\right], X_{i, 0}^{+}\right]_{q^{-6}} \\
& +\left(q^{2}-1\right)\left(1-q^{-4}\right)\left[X_{j, 0}^{+},\left(X_{i, 0}^{+}\right)^{3}\right]_{q^{2}}+q^{-2}\left[\left[\left[X_{j, 0}^{+}, X_{i, 0}^{+}\right]_{q^{-2}}, X_{i, 0}^{+}\right], X_{i, 0}^{+}\right]_{q^{6}} \\
= & \left(q+q^{-1}\right)\left(q^{3}+q^{-3}\right)\left(X_{j, 0}^{+}\left(X_{i, 0}^{+}\right)^{3}-\left(q^{2}+1+q^{-2}\right) X_{i, 0}^{+} X_{j, 0}^{+}\left(X_{i, 0}^{+}\right)^{2}\right. \\
& \left.+\left(q^{2}+1+q^{-2}\right)\left(X_{i, 0}^{+}\right)^{2} X_{j, 0}^{+} X_{i, 0}^{+}-\left(X_{i, 0}^{+}\right)^{3} X_{j, 0}^{+}\right)
\end{aligned}
$$

is an element of $\mathcal{I}_{\text {const }}^{+}\left(X D, X 3^{1}, S 2\right)$, hence $\mathcal{I}_{0}^{+}\left(S_{(i, j)}\right) \subseteq \mathcal{I}_{\text {const }}^{+}\left(X D, X 3^{1}, S 2\right)$. 
Thanks to Corollary 9.5 we obtain $\mathcal{I}_{\text {const }}^{ \pm}\left(S_{(i, j)}\right) \subseteq \mathcal{I}_{\text {const }}^{ \pm}\left(X D, X 3^{1}, S 2\right)$.

Proposition 11.6. If $k=3$ and $i, j \in I_{0}$ are such that $a_{i j}=-3$, then $\mathcal{I}_{\text {const }}^{ \pm}\left(S_{(i, j)}\right)$ $\subseteq \mathcal{I}_{\text {const }}^{ \pm}\left(X 1_{i}, T 3\right)$.

Proof. Let us start from

$$
\left(q^{2}+1\right)\left[\left[X_{j, 0}^{+}, X_{i, 2}^{+}\right]_{q^{3}}, X_{i, 0}^{+}\right]_{q^{-1}}+\left[\left[X_{j, 0}^{+}, X_{i, 1}^{+}\right]_{q^{3}}, X_{i, 1}^{+}\right]_{q},
$$

which is an element of $\mathcal{I}_{\text {const }}^{+}(T 3)$, and remark that

$$
\left[\left[X_{j, 0}^{+}, X_{i, 2}^{+}\right]_{q^{3}}, X_{i, 0}^{+}\right]_{q^{-1}}-q^{2}\left[\left[X_{j, 0}^{+}, X_{i, 0}^{+}\right]_{q^{-3}}, X_{i, 2}^{+}\right]_{q}-\left(q^{2}-1\right)\left[X_{j, 0}^{+},\left(X_{i, 1}^{+}\right)^{2}\right]
$$

belongs to $\mathcal{I}_{\text {const }}^{+}\left(X 1_{i}\right)$; but

$\left(q^{2}+1\right)\left(q^{2}-1\right)\left[X_{j, 0}^{+},\left(X_{i, 1}^{+}\right)^{2}\right]+\left[\left[X_{j, 0}^{+}, X_{i, 1}^{+}\right]_{q^{3}}, X_{i, 1}^{+}\right]_{q}=q^{4}\left[\left[X_{j, 0}^{+}, X_{i, 1}^{+}\right]_{q^{-3}}, X_{i, 1}^{+}\right]_{q^{-1}}$,

so that

$$
\left[\left[X_{j, 0}^{+}, X_{i, 1}^{+}\right]_{q^{-3}}, X_{i, 1}^{+}\right]_{q^{-1}}+\left(1+q^{-2}\right)\left[\left[X_{j, 0}^{+}, X_{i, 0}^{+}\right]_{q^{-3}}, X_{i, 2}^{+}\right]_{q}
$$

lies in $\mathcal{I}_{\text {const }}^{+}\left(X 1_{i}, T 3\right)$, hence

$$
\left[\left[X_{j,-3}^{+}, X_{i, 4}^{+}\right]_{q^{3}}, X_{i, 1}^{+}\right]_{q^{-1}}+\left(1+q^{-2}\right)\left[\left[X_{j,-3}^{+}, X_{i, 3}^{+}\right]_{q^{3}}, X_{i, 2}^{+}\right]_{q}
$$

and (applying $\tilde{t}_{j}^{-1} \tilde{t}_{i}^{2}$ and $q$-commuting by $X_{i, 0}^{+}$)

$$
\left[\left[\left[X_{j, 0}^{+}, X_{i, 2}^{+}\right]_{q^{3}}, X_{i,-1}^{+}\right]_{q^{-1}}+\left(1+q^{-2}\right)\left[\left[X_{j, 0}^{+}, X_{i, 1}^{+}\right]_{q^{3}}, X_{i, 0}^{+}\right]_{q}, X_{i, 0}^{+}\right]_{q^{-3}}
$$

lie in $\mathcal{I}_{\text {const }}^{+}\left(X D, X 1_{i}, T 3\right)$; but

$$
\left[\left[\left[X_{j, 0}^{+}, X_{i, 2}^{+}\right]_{q^{3}}, X_{i,-1}^{+}\right]_{q^{-1}}, X_{i, 0}^{+}\right]_{q^{-3}}-q^{-2}\left[\left[\left[X_{j, 0}^{+}, X_{i, 2}^{+}\right]_{q^{3}}, X_{i, 0}^{+}\right]_{q^{-1}}, X_{i,-1}^{+}\right]_{q}
$$

belongs to $\mathcal{I}_{\text {const }}^{+}\left(X 1_{i}\right)$ by Lemma 11.2,

$$
q^{-2}\left[\left[\left[X_{j, 0}^{+}, X_{i, 2}^{+}\right]_{q^{3}}, X_{i, 0}^{+}\right]_{q^{-1}}, X_{i,-1}^{+}\right]_{q}+\frac{q^{-2}}{q^{2}+1}\left[\left[\left[X_{j, 0}^{+}, X_{i, 1}^{+}\right]_{q^{3}}, X_{i, 1}^{+}\right]_{q}, X_{i,-1}^{+}\right]_{q}
$$

belongs to $\mathcal{I}_{\text {const }}^{+}(T 3)$,

$$
\begin{aligned}
& {\left[\left[\left[X_{j, 0}^{+}, X_{i, 1}^{+}\right]_{q^{3}}, X_{i, 1}^{+}\right]_{q}, X_{i,-1}^{+}\right]_{q}} \\
& \quad-q^{2}\left[\left[\left[X_{j, 0}^{+}, X_{i, 1}^{+}\right]_{q^{3}}, X_{i,-1}^{+}\right]_{q^{-1}}, X_{i, 1}^{+}\right]_{q^{-1}}-\left(q^{2}-1\right)\left[\left[X_{j, 0}^{+}, X_{i, 1}^{+}\right]_{q^{3}},\left(X_{i, 0}^{+}\right)^{2}\right]
\end{aligned}
$$

belongs to $\mathcal{I}_{\text {const }}^{+}\left(X 1_{i}\right)$ again by Lemma 11.2 ,

$$
q^{2}\left[\left[\left[X_{j, 0}^{+}, X_{i, 1}^{+}\right]_{q^{3}}, X_{i,-1}^{+}\right]_{q^{-1}}, X_{i, 1}^{+}\right]_{q^{-1}}+\frac{q^{2}}{q^{2}+1}\left[\left[\left[X_{j, 0}^{+}, X_{i, 0}^{+}\right]_{q^{3}}, X_{i, 0}^{+}\right]_{q}, X_{i, 1}^{+}\right]_{q^{-1}}
$$

belongs to $\mathcal{I}_{\text {const }}^{+}(T 3)$ and

$$
\left[\left[\left[X_{j, 0}^{+}, X_{i, 0}^{+}\right]_{q^{3}}, X_{i, 0}^{+}\right]_{q}, X_{i, 1}^{+}\right]_{q^{-1}}-q^{-4}\left[\left[\left[X_{j, 0}^{+}, X_{i, 1}^{+}\right]_{q^{3}}, X_{i, 0}^{+}\right]_{q^{5}}, X_{i, 0}^{+}\right]_{q^{3}}
$$

belongs to $\mathcal{I}_{\text {const }}^{+}\left(X 1_{i}\right)$ (by Lemma 11.2). So we can conclude that 


$$
\begin{aligned}
\frac{q^{-4}}{\left(q^{2}+1\right)^{2}}\left[\left[\left[X_{j, 0}^{+}, X_{i, 1}^{+}\right]_{q^{3}}, X_{i, 0}^{+}\right]_{q^{5}}, X_{i, 0}^{+}\right]_{q^{3}}-\frac{1-q^{-2}}{q^{2}+1}\left[\left[X_{j, 0}^{+}, X_{i, 1}^{+}\right]_{q^{3}},\left(X_{i, 0}^{+}\right)^{2}\right] \\
+\left(1+q^{-2}\right)\left[\left[\left[X_{j, 0}^{+}, X_{i, 1}^{+}\right]_{q^{3}}, X_{i, 0}^{+}\right]_{q}, X_{i, 0}^{+}\right]_{q^{-3}} \\
=\frac{\left(q^{2}+1+q^{-2}\right)^{2}}{\left(q^{2}+1\right)^{2}}\left[\left[\left[X_{j, 0}^{+}, X_{i, 1}^{+}\right]_{q^{3}}, X_{i, 0}^{+}\right]_{q}, X_{i, 0}^{+}\right]_{q^{-1}}
\end{aligned}
$$

lies in $\mathcal{I}_{\text {const }}^{+}\left(X D, X 1_{i}, T 3\right)$.

Thus $\left[\left[\left[\left[X_{j, 0}^{+}, X_{i, 1}^{+}\right]_{q^{3}}, X_{i, 0}^{+}\right]_{q}, X_{i, 0}^{+}\right]_{q^{-1}}, X_{i,-1}^{+}\right]_{q^{3}} \in \mathcal{I}_{\text {const }}^{+}\left(X 1_{i}, T 3\right)$; but

$$
\begin{aligned}
{\left[\left[\left[\left[X_{j, 0}^{+}, X_{i, 1}^{+}\right]_{q^{3}}, X_{i, 0}^{+}\right]_{q}, X_{i, 0}^{+}\right]_{q^{-1}}, X_{i,-1}^{+}\right]_{q^{3}} } & \\
& -q^{4}\left[\left[\left[\left[X_{j, 0}^{+}, X_{i, 1}^{+}\right]_{q^{3}}, X_{i,-1}^{+}\right]_{q^{-1}}, X_{i, 0}^{+}\right]_{q^{-1}}, X_{i, 0}^{+}\right]_{q^{-3}}
\end{aligned}
$$

belongs to $\mathcal{I}_{\text {const }}^{ \pm}\left(X 1_{i}\right)$ and since

$$
\left(q^{2}+1\right)\left[\left[X_{j, 0}^{+}, X_{i, 1}^{+}\right]_{q^{3}}, X_{i,-1}^{+}\right]_{q^{-1}}+\left[\left[X_{j, 0}^{+}, X_{i, 0}^{+}\right]_{q^{3}}, X_{i, 0}^{+}\right]_{q} \in \mathcal{I}_{\text {const }}^{+}(T 3)
$$

it follows that

$$
\left[\left[\left[\left[X_{j, 0}^{+}, X_{i, 0}^{+}\right]_{q^{3}}, X_{i, 0}^{+}\right]_{q}, X_{i, 0}^{+}\right]_{q^{-1}}, X_{i, 0}^{+}\right]_{q^{-3}} \in \mathcal{I}_{\text {const }}^{+}(X 1, T 3),
$$

so that $\mathcal{I}_{0}^{+}\left(S_{(i, j)}\right) \subseteq \mathcal{I}_{\text {const }}^{+}(X 1, T 3)$. Hence $\mathcal{I}_{\text {const }}^{ \pm}\left(S_{(i, j)}\right) \subseteq \mathcal{I}_{\text {const }}^{ \pm}(X 1, T 3)$.

Corollary 11.7. $\mathcal{I}_{\text {const }}(S)=0$ in $\mathcal{U}_{q}^{\text {Dr }}$.

Proof. The claim is a straightforward consequence of Remark 11.1 and Propositions 11.3, 11.5, 11.6.

We are now able to prove that the quantum algebra $\mathcal{U}_{q}^{\text {fin }}$ of finite type is mapped in $\mathcal{U}_{q}^{\text {Dr }}$, which was not otherwise clear.

Definition 11.8. Let $\phi: \mathcal{U}_{q}^{\text {fin }} \rightarrow \mathcal{U}_{q}^{\text {Dr }}$ be the $\mathbb{C}(q)$-homomorphism given by

$$
K_{i}^{ \pm 1} \mapsto k_{i}^{ \pm 1}, \quad E_{i} \mapsto X_{i, 0}^{+}, \quad F_{i} \mapsto X_{i, 0}^{-} \quad\left(i \in I_{0}\right) .
$$

Remark 11.9. $\phi$ is well defined.

Proof. This is a straightforward consequence of Corollary 11.7 (and of $(C U K)$, $\left.(C K),\left(K X^{ \pm}\right),(X X E)\right)$.

We shall now complete the study of the ideal generated by the Serre relations.

Remark 11.10. $\mathcal{I}_{\text {const }}^{ \pm}(X D) \subseteq \mathcal{I}_{\text {const }}^{ \pm}(S)$. If $n>1$ then

$$
\mathcal{I}_{\text {const }}^{ \pm}(X 1), \mathcal{I}_{\text {const }}^{ \pm}(X 2), \mathcal{I}_{\text {const }}^{ \pm}\left(X 3^{1}\right), \mathcal{I}_{\text {const }}^{ \pm}\left(X 3^{-1}\right) \subseteq \mathcal{I}_{\text {const }}^{ \pm}(S) \text {. }
$$

Proof. That $\mathcal{I}_{\text {const }}^{ \pm}(X D) \subseteq \mathcal{I}_{\text {const }}^{ \pm}(S)$ follows from Proposition 10.4 and from Remark 11.1. 
That $\mathcal{I}_{\text {const }}^{ \pm}(X 1) \subseteq \mathcal{I}_{\text {const }}^{ \pm}(S)$ is a consequence of Lemma 10.5 and of Corollary 9.5 (see also Corollary 10.6).

Finally that $\mathcal{I}_{\text {const }}^{ \pm}\left(X 3^{1}\right) \subseteq \mathcal{I}_{\text {const }}^{ \pm}(S)$ follows again from Lemma 10.5 and from Corollary 9.5 , once one notices that $\left(X_{\tilde{n}}^{(k)}, i, j\right)=\left(A_{2 n}^{(2)}, 1,2\right)$ implies $q_{i}=q, b_{j i \tilde{d}_{i j}}$ $\neq 0$ and $b_{i i \tilde{d}_{i}}=[2]_{q}[3]_{q}$.

From this it follows that $\mathcal{I}_{\text {const }}^{ \pm}(X 2), \mathcal{I}_{\text {const }}^{ \pm}\left(X 3^{-1}\right) \subseteq \mathcal{I}_{\text {const }}^{ \pm}(S)$ (see Proposition 10.1).

Corollary 11.11. (i) $\mathcal{I}^{ \pm}(S)=\mathcal{I}_{\text {const }}^{ \pm}(S)$ (see Corollary 9.17(iii) and Remark 11.10);

(ii) $\mathcal{I}(S)=0$ in $\mathcal{U}_{q}^{\mathrm{Dr}}$ (see Corollary 11.7).

Remark 11.12. If $k>1$ and $X_{\tilde{n}} \neq A_{2 n}$ then $\mathcal{I}_{\text {const }}^{ \pm}(S k) \subseteq \mathcal{I}_{\text {const }}^{ \pm}(S) \Leftrightarrow \mathcal{I}_{\text {const }}^{ \pm}(T k)$ $\subseteq \mathcal{I}_{\text {const }}^{ \pm}(S)$.

Proof. Of course we can suppose $n>1$; then the claim depends on the fact that $\left(\mathcal{I}_{\text {const }}^{ \pm}(X 1), \mathcal{I}_{\text {const }}^{ \pm}(S k)\right)=\left(\mathcal{I}_{\text {const }}^{ \pm}(X 1), \mathcal{I}_{\text {const }}^{ \pm}(T k)\right)$ (see Remark 5.8) and that $\mathcal{I}_{\text {const }}^{ \pm}(X 1) \subseteq \mathcal{I}_{\text {const }}^{ \pm}(S)$ (see Remark 11.10)

Proposition 11.13. $\mathcal{I}_{\text {const }}^{ \pm}(S 2) \subseteq \mathcal{I}_{\text {const }}^{ \pm}(S)$.

Proof. Suppose that $k=2$ and let $i, j \in I_{0}$ be such that $a_{i j}=-2$. Then $\left[\left[\left[X_{j, 0}^{+}, X_{i, 0}^{+}\right]_{q^{2}}, X_{i, 0}^{+}\right], X_{i, 0}^{+}\right]_{q^{-2}}$ is an element of $\mathcal{I}_{\text {const }}^{+}(S)$, so that

$$
\begin{aligned}
\mathcal{I}_{\text {const }}^{+} & (S) \ni\left[\left[\left[\left[X_{j, 0}^{+}, X_{i, 0}^{+}\right]_{q^{2}}, X_{i, 0}^{+}\right], X_{i, 0}^{+}\right]_{q^{-2}}, X_{i, 1}^{-}\right] \\
= & {\left[\left[\left[X_{j, 0}^{+}, C^{-1} k_{i} H_{i, 1}\right]_{q^{2}}, X_{i, 0}^{+}\right], X_{i, 0}^{+}\right]_{q^{-2}}+\left[\left[\left[X_{j, 0}^{+}, X_{i, 0}^{+}\right]_{q^{2}}, C^{-1} k_{i} H_{i, 1}\right], X_{i, 0}^{+}\right]_{q^{-2}} } \\
& +\left[\left[\left[X_{j, 0}^{+}, X_{i, 0}^{+}\right]_{q^{2}}, X_{i, 0}^{+}\right], C^{-1} k_{i} H_{i, 1}\right]_{q^{-2}} \\
= & -C^{-1} k_{i}\left(q^{2}\left[\left[\left[H_{i, 1}, X_{j, 0}^{+}\right], X_{i, 0}^{+}\right]_{q^{-2}}, X_{i, 0}^{+}\right]_{q^{-4}}+\left[\left[\left[H_{i, 1}, X_{j, 0}^{+}\right], X_{i, 0}^{+}\right]_{q^{2}}, X_{i, 0}^{+}\right]_{q^{-4}}\right. \\
& +\left[\left[X_{j, 0}^{+},\left[H_{i, 1}, X_{i, 0}^{+}\right]\right]_{q^{2}}, X_{i, 0}^{+}\right]_{q^{-4}}+q^{-2}\left[\left[\left[H_{i, 1}, X_{j, 0}^{+}\right], X_{i, 0}^{+}\right]_{q^{2}}, X_{i, 0}^{+}\right] \\
& \left.+q^{-2}\left[\left[X_{j, 0}^{+},\left[H_{i, 1}, X_{i, 0}^{+}\right]\right]_{q^{2}}, X_{i, 0}^{+}\right]+q^{-2}\left[\left[X_{j, 0}^{+}, X_{i, 0}^{+}\right]_{q^{2}},\left[H_{i, 1}, X_{i, 0}^{+}\right]\right]\right) \\
= & -C^{-1} k_{i}\left(b _ { i j 1 } \left(q^{2}\left[\left[X_{j, 1}^{+}, X_{i, 0}^{+}\right]_{q^{-2}}, X_{i, 0}^{+}\right]_{q^{-4}}+\left[\left[X_{j, 1}^{+}, X_{i, 0}^{+}\right]_{q^{2}}, X_{i, 0}^{+}\right]_{q^{-4}}\right.\right. \\
& \left.+q^{-2}\left[\left[X_{j, 1}^{+}, X_{i, 0}^{+}\right]_{q^{2}}, X_{i, 0}^{+}\right]\right)+b_{i i 1}\left(\left[\left[X_{j, 0}^{+}, X_{i, 1}^{+}\right]_{q^{2}}, X_{i, 0}^{+}\right]_{q^{-4}}\right. \\
& \left.\left.+q^{-2}\left[\left[X_{j, 0}^{+}, X_{i, 1}^{+}\right]_{q^{2}}, X_{i, 0}^{+}\right]+q^{-2}\left[\left[X_{j, 0}^{+}, X_{i, 0}^{+}\right]_{q^{2}}, X_{i, 1}^{+}\right]\right)\right) \\
= & -C^{-1} k_{i}\left([3]_{q} b_{i j 1}\left(X_{j, 1}^{+}\left(X_{i, 0}^{+}\right)^{2}-\left(q^{-2}+1\right) X_{i, 0}^{+} X_{j, 1}^{+} X_{i, 0}^{+}+q^{-2}\left(X_{i, 0}^{+}\right)^{2} X_{j, 1}^{+}\right)\right. \\
& +[3]_{q} b_{i i 1}\left(q^{-2} X_{j, 0}^{+} X_{i, 1}^{+} X_{i, 0}^{+}-X_{i, 1}^{+} X_{j, 0}^{+} X_{i, 0}^{+}-q^{-2} X_{i, 0}^{+} X_{j, 0}^{+} X_{i, 1}^{+}+X_{i, 0}^{+} X_{i, 1}^{+} X_{j, 0}^{+}\right) \\
& \left.+b_{i i 1}\left(-q^{-4} X_{j, 0}^{+}\left[X_{i, 1}^{+}, X_{i, 0}^{+}\right]_{q^{2}}+\left[X_{i, 1}^{+}, X_{i, 0}^{+}\right]_{q^{2}} X_{j, 0}^{+}\right)\right) \\
= & -C^{-1} k_{i}\left([3]_{q} b_{i j 1}\left[\left[X_{j, 1}^{+}, X_{i, 0}^{+}\right]_{q^{-2}}, X_{i, 0}^{+}\right]\right. \\
& \left.+q^{-2}[3]_{q} b_{i i 1}\left[\left[X_{j, 0}^{+}, X_{i, 1}^{+}\right]_{q^{2}}, X_{i, 0}^{+}\right]+b_{i i 1}\left[\left[X_{i, 1}^{+}, X_{i, 0}^{+}\right]_{q^{2}}, X_{j, 0}^{+}\right]_{q^{-4}}\right) .
\end{aligned}
$$


Now notice that if $X_{\tilde{n}} \neq A_{2 n}$ we have $b_{i i 1} \neq 0,\left[X_{i, 1}^{+}, X_{i, 0}^{+}\right]_{q^{2}} \in \mathcal{I}_{\text {const }}^{+}(S)$ (see Remark 11.10) and $\tilde{d}_{j}=2$, hence $b_{i j 1}=0$; we can conclude that $\left[\left[X_{j, 0}^{+}, X_{i, 1}^{+}\right]_{q^{2}}, X_{i, 0}^{+}\right]$ is an element of $\mathcal{I}_{\text {const }}^{+}(S)$, so that $\mathcal{I}_{\text {const }}^{+}(T 2) \subseteq \mathcal{I}_{\text {const }}^{+}(S)$ (see Corollary 9.5), which, thanks to Remark 11.12, is equivalent to $\mathcal{I}_{\text {const }}^{+}(S 2) \subseteq \mathcal{I}_{\text {const }}^{+}(S)$.

On the other hand, if $X_{\tilde{n}}=A_{2 n}$ we have $b_{i i 1}=[2]_{q}[3]_{q}, \tilde{d}_{j}=1, b_{i j 1}=-[2]_{q}$ and $\left[X_{j, 1}^{+}, X_{i, 0}^{+}\right]_{q^{-2}}+\left[X_{i, 1}^{+}, X_{j, 0}^{+}\right]_{q^{-2}} \in \mathcal{I}_{\text {const }}^{+}(S)$ (see Remark 11.10); then

$$
\left(q^{2}+q^{-2}\right)\left[\left[X_{j, 0}^{+}, X_{i, 1}^{+}\right]_{q^{2}}, X_{i, 0}^{+}\right]+q^{2}\left[\left[X_{i, 1}^{+}, X_{i, 0}^{+}\right]_{q^{2}}, X_{j, 0}^{+}\right]_{q^{-4}}
$$

is an element of $\mathcal{I}_{\text {const }}^{+}(S)$, that is, $\mathcal{I}_{\text {const }}^{+}(S 2) \subseteq \mathcal{I}_{\text {const }}^{+}(S)$.

In both cases using Corollary 9.5 we get $\mathcal{I}_{\text {const }}^{ \pm}(S 2) \subseteq \mathcal{I}_{\text {const }}^{ \pm}(S)$.

Proposition 11.14. $\mathcal{I}_{\text {const }}^{ \pm}(T 3) \subseteq \mathcal{I}_{\text {const }}^{ \pm}(S)$.

Proof. Let $k=3\left(X_{\tilde{n}}^{(k)}=D_{4}^{(3)}\right)$ and $i, j \in I_{0}$ be such that $a_{i j}=-3(i=1, j=2)$. Then $\left[\left[\left[\left[X_{j, 0}^{+}, X_{i, 0}^{+}\right]_{q^{3}}, X_{i, 0}^{+}\right]_{q}, X_{i, 0}^{+}\right]_{q^{-1}}, X_{i, 0}^{+}\right]_{q^{-3}}$ is an element of $\mathcal{I}_{\text {const }}^{+}(S)$, so that, recalling that $b_{i j 1}=0$ and $b_{i i 1}=[2]_{q}$,

$$
\begin{aligned}
\mathcal{I}_{\text {const }}^{+}(S) & \ni\left[\left[\left[\left[\left[X_{j, 0}^{+}, X_{i, 0}^{+}\right]_{q^{3}}, X_{i, 0}^{+}\right]_{q}, X_{i, 0}^{+}\right]_{q^{-1}}, X_{i, 0}^{+}\right]_{q^{-3}}, X_{i, 1}^{-}\right] \\
= & {\left[\left[\left[\left[X_{j, 0}^{+}, C^{-1} k_{i} H_{i, 1}\right]_{q^{3}}, X_{i, 0}^{+}\right]_{q}, X_{i, 0}^{+}\right]_{q^{-1}}, X_{i, 0}^{+}\right]_{q^{-3}} } \\
& +\left[\left[\left[\left[X_{j, 0}^{+}, X_{i, 0}^{+}\right]_{q^{3}}, C^{-1} k_{i} H_{i, 1}\right]_{q}, X_{i, 0}^{+}\right]_{q^{-1}}, X_{i, 0}^{+}\right]_{q^{-3}} \\
& +\left[\left[\left[\left[X_{j, 0}^{+}, X_{i, 0}^{+}\right]_{q^{3}}, X_{i, 0}^{+}\right]_{q}, C^{-1} k_{i} H_{i, 1}\right]_{q^{-1}}, X_{i, 0}^{+}\right]_{q^{-3}} \\
& +\left[\left[\left[\left[X_{j, 0}^{+}, X_{i, 0}^{+}\right]_{q^{3}}, X_{i, 0}^{+}\right]_{q}, X_{i, 0}^{+}\right]_{q^{-1}}, C^{-1} k_{i} H_{i, 1}\right]_{q^{-3}} \\
= & -C^{-1} k_{i}\left(q^{3}\left[\left[\left[\left[H_{i, 1}, X_{j, 0}^{+}\right], X_{i, 0}^{+}\right]_{q^{-1}}, X_{i, 0}^{+}\right]_{q^{-3}}, X_{i, 0}^{+}\right]_{q^{-5}}\right. \\
& +q\left[\left[\left[H_{i, 1},\left[X_{j, 0}^{+}, X_{i, 0}^{+}\right]_{q^{3}}\right], X_{i, 0}^{+}\right]_{q^{-3}}, X_{i, 0}^{+}\right]_{q^{-5}} \\
& +q^{-1}\left[\left[H_{i, 1},\left[\left[X_{j, 0}^{+}, X_{i, 0}^{+}\right]_{q^{3}}, X_{i, 0}^{+}\right]_{q}\right], X_{i, 0}^{+}\right]_{q^{-5}} \\
& \left.+q^{-3}\left[H_{i, 1},\left[\left[\left[X_{j, 0}^{+}, X_{i, 0}^{+}\right]_{q^{3}}, X_{i, 0}^{+}\right]_{q}, X_{i, 0}^{+}\right]_{q^{-1}}\right]\right) \\
= & -[2]_{q} C^{-1} k_{i}\left(q\left[\left[\left[X_{j, 0}^{+}, X_{i, 1}^{+}\right]_{q^{3}}, X_{i, 0}^{+}\right]_{q^{-3}}, X_{i, 0}^{+}\right]_{q^{-5}}\right. \\
& +q^{-1}\left[\left[\left[X_{j, 0}^{+}, X_{i, 1}^{+}\right]_{q^{3}}, X_{i, 0}^{+}\right]_{q}, X_{i, 0}^{+}\right]_{q^{-5}} \\
& \left.+q^{-1}\left[\left[\left[X_{j, 0}^{+}, X_{i, 0}^{+}\right]_{q^{3}}, X_{i, 1}^{+}\right]_{q}, X_{i, 0}^{+}\right]_{q^{-5}}+q^{-3}\left[\left[\left[X_{j, 0}^{+}, X_{i, 1}^{+}\right]_{q^{3}}, X_{i, 0}^{+}\right]_{q}, X_{i, 0}^{+}\right]_{q^{-1}}\right) \\
& \left.\left.+q^{-3}\left[\left[\left[X_{j, 0}^{+}, X_{i, 0}^{+}\right]_{q^{3}}, X_{i, 1}^{+}\right]_{q}, X_{i, 0}^{+}\right]_{q^{-1}}\right)+q^{-3}\left[\left[\left[X_{j, 0}^{+}, X_{i, 0}^{+}\right]_{q^{3}}, X_{i, 0}^{+}\right]_{q}, X_{i, 1}^{+}\right]_{q^{-1}}\right)
\end{aligned}
$$

then, thanks to Remarks 11.10 and 11.2, we infer that

$$
\begin{array}{r}
q\left[\left[\left[X_{j, 0}^{+}, X_{i, 1}^{+}\right]_{q^{3}}, X_{i, 0}^{+}\right]_{q^{-3}}, X_{i, 0}^{+}\right]_{q^{-5}}+q^{-1}\left[\left[\left[X_{j, 0}^{+}, X_{i, 1}^{+}\right]_{q^{3}}, X_{i, 0}^{+}\right]_{q}, X_{i, 0}^{+}\right]_{q^{-5}} \\
\left.+q^{-3}\left[\left[\left[X_{j, 0}^{+}, X_{i, 1}^{+}\right]_{q^{3}}, X_{i, 0}^{+}\right]_{q^{5}}, X_{i, 0}^{+}\right]_{q^{-5}}+q^{-3}\left[\left[\left[X_{j, 0}^{+}, X_{i, 1}^{+}\right]_{q^{3}}, X_{i, 0}^{+}\right]_{q}, X_{i, 0}^{+}\right]_{q^{-1}}\right) \\
\left.+q^{-5}\left[\left[\left[X_{j, 0}^{+}, X_{i, 1}^{+}\right]_{q^{3}}, X_{i, 0}^{+}\right]_{q^{5}}, X_{i, 0}^{+}\right]_{q^{-1}}\right)+q^{-7}\left[\left[\left[X_{j, 0}^{+}, X_{i, 0}^{+}\right]_{q^{3}}, X_{i, 0}^{+}\right]_{q^{5}}, X_{i, 1}^{+}\right]_{q^{3}} \\
=q^{-3}\left(q^{2}+q^{-2}\right)[3]_{q}\left[\left[\left[X_{j, 0}^{+}, X_{i, 1}^{+}\right]_{q^{3}}, X_{i, 0}^{+}\right]_{q}, X_{i, 0}^{+}\right]_{q^{-1}}
\end{array}
$$


belongs to $\mathcal{I}_{\text {const }}^{+}(S)$; then so does

$$
\begin{aligned}
& {\left[\left[\left[\left[X_{j, 0}^{+}, X_{i, 1}^{+}\right]_{q^{3}}, X_{i, 0}^{+}\right]_{q^{-1}}, X_{i, 0}^{+}\right]_{q}, X_{i,-1}^{-}\right] } \\
&= {\left[\left[\left[X_{j, 0}^{+}, \frac{C k_{i}-C^{-1} k_{i}^{-1}}{q-q^{-1}}\right]_{q^{3}}, X_{i, 0}^{+}\right]_{q^{-1}}, X_{i, 0}^{+}\right]_{q} } \\
&+\left[\left[\left[X_{j, 0}^{+}, X_{i, 1}^{+}\right]_{q^{3}}, k_{i}^{-1} H_{i,-1}\right]_{q^{-1}}, X_{i, 0}^{+}\right]_{q}+\left[\left[\left[X_{j, 0}^{+}, X_{i, 1}^{+}\right]_{q^{3}}, X_{i, 0}^{+}\right]_{q^{-1}}, k_{i}^{-1} H_{i,-1}\right]_{q} \\
&= k_{i}^{-1}\left([3]_{q} C^{-1}\left[\left[X_{j, 0}^{+}, X_{i, 0}^{+}\right]_{q}, X_{i, 0}^{+}\right]_{q}\right. \\
&-q^{-1}\left[\left[\left[H_{i,-1},\left[X_{j, 0}^{+}, X_{i, 1}^{+}\right]_{q^{3}}\right], X_{i, 0}^{+}\right]_{q^{3}}-q\left[H_{i,-1},\left[\left[X_{j, 0}^{+}, X_{i, 1}^{+}\right]_{q^{3}}, X_{i, 0}^{+}\right]_{q^{-1}}\right]\right) \\
&= C^{-1} k_{i}^{-1}\left([3]_{q}\left[\left[X_{j, 0}^{+}, X_{i, 0}^{+}\right]_{q^{3}}, X_{i, 0}^{+}\right]_{q}-q^{-1}[2]_{q}\left[\left[X_{j, 0}^{+}, X_{i, 0}^{+}\right]_{q^{3}}, X_{i, 0}^{+}\right]_{q^{3}}\right. \\
&\left.-q[2]_{q}\left[\left[X_{j, 0}^{+}, X_{i, 0}^{+}\right]_{q^{3}}, X_{i, 0}^{+}\right]_{q^{-1}}-q[2]_{q}\left[\left[X_{j, 0}^{+}, X_{i, 1}^{+}\right]_{q^{3}}, X_{i,-1}^{+}\right]_{q^{-1}}\right) \\
&=-C^{-1} k_{i}^{-1}\left(\left[\left[X_{j, 0}^{+}, X_{i, 0}^{+}\right]_{q^{3}}, X_{i, 0}^{+}\right]_{q}+q[2]_{q}\left[\left[X_{j, 0}^{+}, X_{i, 1}^{+}\right]_{q^{3}}, X_{i,-1}^{+}\right]_{q^{-1}}\right) ;
\end{aligned}
$$

hence $\mathcal{I}_{0}^{+}(T 3) \subseteq \mathcal{I}_{\text {const }}^{+}(S)$ and, using Corollary $9.5, \mathcal{I}_{\text {const }}^{ \pm}(T 3) \subseteq \mathcal{I}_{\text {const }}^{ \pm}(S)$.

Corollary 11.15. $\mathcal{I}_{\text {const }}^{ \pm}(S 3) \subseteq \mathcal{I}_{\text {const }}^{ \pm}(S)$.

Proof. This follows from Remark 11.12 and from Proposition 11.14.

Corollary 11.16. If $n>1$ then $\mathcal{I}_{\text {const }}^{ \pm}\left(X D, X 1, X 2, X 3^{ \pm 1}, S U L, S 2, S 3\right)=$ $\mathcal{I}_{\text {const }}^{ \pm}(S)$.

Proof. This follows from Corollary 11.7, Remarks 11.1 and 11.10, Proposition 11.13 and Corollary 11.15.

Remark 11.17. In $\mathcal{U}_{q}^{\operatorname{Dr}}\left(D_{4}^{(3)}\right)$ we have $\left[\left[\left[X_{j, 0}^{+}, X_{i, 1}^{+}\right]_{q^{3}}, X_{i, 0}^{+}\right]_{q}, X_{i, 0}^{+}\right]_{q^{-1}}=0$.

Proof. See the proof of Proposition 11.14.

Theorem 11.18. (i) $\mathcal{U}_{q}^{\mathrm{Dr}}\left(A_{1}^{(1)}\right)=\tilde{\mathcal{U}}_{q}^{\mathrm{Dr}}\left(A_{1}^{(1)}\right) / \mathcal{I}_{\text {const }}(X 1)$;

(ii) $\mathcal{U}_{q}^{\operatorname{Dr}}\left(A_{2}^{(2)}\right)=\tilde{\mathcal{U}}_{q}^{\operatorname{Dr}}\left(A_{2}^{(2)}\right) / \mathcal{I}_{\text {const }}\left(X 3^{1}\right)$;

(iii) $\mathcal{U}_{q}^{\operatorname{Dr}}\left(X_{\tilde{n}}^{(k)}\right)=\tilde{\mathcal{U}}_{q}^{\operatorname{Dr}}\left(X_{\tilde{n}}^{(k)}\right) / \mathcal{I}_{\text {const }}(S)$ if $n>1$ (that is, $\left.X_{\tilde{n}}^{(k)} \neq A_{1}^{(1)}, A_{2}^{(2)}\right)$.

Proof. The claims follow from Theorem 10.8 and Corollary 11.16.

Corollary 11.19. Let $U$ be a $\mathbb{C}(q)$-algebra, $t_{i}^{(U)}\left(i \in I_{0}\right)$ be $\mathbb{C}(q)$-automorphisms of $U, \Omega^{(U)}$ be a $\mathbb{C}$-antilinear antiautomorphism of $U$, and $\tilde{f}: \tilde{\mathcal{U}}_{q}^{\mathrm{Dr}}\left(X_{\tilde{n}}^{(k)}\right) \rightarrow U$ be a homomorphism of $\mathbb{C}(q)$-algebras such that $\tilde{f} \circ \tilde{t}_{i}=t_{i}^{(U)} \circ \tilde{f}$ for all $i \in I_{0}$ and $\tilde{f} \circ \tilde{\Omega}=\Omega^{(U)} \circ \tilde{f} . I f:$

(i) $\tilde{f}\left(\mathcal{I}_{0}^{+}(X 1)\right)=0$ in case $X_{\tilde{n}}^{(k)}=A_{1}^{(1)}$;

(ii) $\tilde{f}\left(\mathcal{I}_{0}^{+}\left(X 3^{1}\right)\right)=0$ in case $X_{\tilde{n}}^{(k)}=A_{2}^{(2)}$;

(iii) $\tilde{f}\left(\mathcal{I}_{0}^{+}(S)\right)=0$ in case $X_{\tilde{n}}^{(k)} \neq A_{1}^{(1)}, A_{2}^{(2)}$, 
then $\tilde{f}$ induces $f: \mathcal{U}_{q}^{\operatorname{Dr}}\left(X_{\tilde{n}}^{(k)}\right) \rightarrow U$ and we have $f \circ t_{i}=t_{i}^{(U)} \circ f$ for all $i \in I_{0}$, and $f \circ \Omega=\Omega^{(U)} \circ f$.

Proof. Since the hypotheses imply that $\operatorname{ker}(\tilde{f})$ is a $t_{i}$-stable (for all $i \in I_{0}$ ), $\tilde{\Omega}$-stable ideal of $\mathcal{U}_{q}^{\operatorname{Dr}}\left(X_{\tilde{n}}^{(k)}\right)$, the claim is an immediate consequence of Theorem 11.18 and of Corollary 9.5.

Remark 11.20. It is useful to compare the results of this section with those of $\S 10$. The simplification of the relations given in $\S 10$ (Theorem 10.8 and Corollary 10.9) provides a minimal set of relations of lowest "degree" (where the degree of $X_{i_{1}, r_{1}}$. $\ldots X_{i_{h}, r_{h}}$ is meant to be $\left.h\right)$; this minimality can be often useful, in spite of the more complicated appearance of relations like $\left(S 2^{ \pm}\right)$compared with the simple and familiar Serre relations. On the other hand the advantage of the Serre relations is evident in all those cases, like the application of Theorem 11.18 and Corollary 11.19 given in $\S 12$, when the Serre relations play a central role; in this connection recall that the Serre relations are the minimal degree relations defining the positive part of $\mathcal{U}_{q}^{\text {fin }}$ (see Definition 11.8 and Remark 11.9, and recall [L]).

\section{§12. The homomorphism $\psi$ from $\mathcal{U}_{q}^{\text {Dr }}$ onto $\mathcal{U}_{q}^{\mathrm{DJ}}$}

This section is devoted to exhibiting a homomorphism $\psi: \mathcal{U}_{q}^{\mathrm{Dr}} \rightarrow \mathcal{U}_{q}^{\mathrm{DJ}}$ and proving that it is surjective.

Notation 12.1. In the following, $o: I_{0} \rightarrow\{ \pm 1\}$ will be a map such that:

(a) $a_{i j} \neq 0 \Rightarrow o(i) o(j)=-1$ (see [Be] for the untwisted case);

(b) in the twisted case different from $A_{2 n}^{(2)}, a_{i j}=-2 \Rightarrow o(i)=1$.

Remark 12.2. A map $o$ as in Notation 12.1 exists and is:

(i) determined up to sign in the untwisted case and in cases $A_{2 n}^{(2)}$ and $D_{4}^{(3)}$;

(ii) uniquely determined in cases $A_{2 n-1}^{(2)}$ and $E_{6}^{(2)}$.

Definition 12.3. Let $\tilde{\psi}=\tilde{\psi}_{X_{\tilde{n}}^{(k)}}: \tilde{\mathcal{U}}_{q}^{\mathrm{Dr}}\left(X_{\tilde{n}}^{(k)}\right) \rightarrow \mathcal{U}_{q}^{\mathrm{DJ}}\left(X_{\tilde{n}}^{(k)}\right)$ be the $\mathbb{C}(q)$-algebra homomorphism defined on the generators as follows:

$$
\begin{gathered}
C^{ \pm 1} \mapsto K_{\delta}^{ \pm 1}, \quad k_{i}^{ \pm 1} \mapsto K_{i}^{ \pm 1} \quad\left(i \in I_{0}\right), \\
X_{i, \tilde{d}_{i} r}^{+} \mapsto o(i)^{r} T_{\lambda_{i}}^{-r}\left(E_{i}\right), \quad X_{i, \tilde{d}_{i} r}^{-} \mapsto o(i)^{r} T_{\lambda_{i}}^{r}\left(F_{i}\right) \quad\left(i \in I_{0}, r \in \mathbb{Z}\right), \\
H_{i, \tilde{d}_{i} r} \mapsto\left\{\begin{array}{ll}
o(i)^{r} E_{\left(\tilde{d}_{i} r \delta, i\right)} & \text { if } r>0 \\
o(i)^{r} F_{\left(-\tilde{d}_{i} r \delta, i\right)} & \text { if } r<0
\end{array} \quad\left(i \in I_{0}, r \in \mathbb{Z} \backslash\{0\}\right) .\right.
\end{gathered}
$$


Proposition 12.4. (i) $\tilde{\psi}$ is well defined;

(ii) $\tilde{\psi} \circ \tilde{\Omega}=\Omega \circ \tilde{\psi}$;

(iii) $\tilde{\psi} \circ \tilde{t}_{i}=T_{\lambda_{i}} \circ \tilde{\psi}$ for all $i \in I_{0}$;

(iv) $\tilde{\psi} \circ \tilde{\phi}_{i}=\varphi_{i} \circ \tilde{\psi}$ for all $i \in I_{0}$;

(v) $\tilde{\psi} \circ \tilde{\Theta}=\left(\Omega \Xi T_{1}\right) \circ \tilde{\psi}$ in cases $A_{1}^{(1)}$ and $A_{2}^{(2)}$.

Proof. (i) The relations $\left(Z X^{ \pm}\right),(C U K),(C K)$ and $\left(K X^{ \pm}\right)$are obviously preserved by $\tilde{\psi}$; also $(X X)$ (see [Be] and [Da]) and $\left(H X L^{ \pm}\right)$hold in $\mathcal{U}_{q}$ : it is enough to notice that for all $i, j \in I_{0}$ and $r \in \mathbb{Z}$ such that $\max \left\{\tilde{d}_{i}, \tilde{d}_{j}\right\} \mid r$ we have $b_{i j r}=(o(i) o(j))^{\tilde{r}} x_{i j r}$ where $\tilde{r}=r / \max \left\{\tilde{d}_{i}, \tilde{d}_{j}\right\}$ and

$x_{i j r}= \begin{cases}(o(i) o(j))^{r} \frac{\left[r a_{i j}\right]_{q_{i}}}{r} & \text { if } k=1, \text { or } X_{\tilde{n}}^{(k)}=A_{2 n}^{(2)} \text { and }(i, j) \neq(1,1), \\ \frac{[2 r]_{q}}{r}\left(q^{2 r}+(-1)^{r-1}+q^{-2 r}\right) & \text { if }\left(X_{\tilde{n}}^{(k)}, i, j\right)=\left(A_{2 n}^{(2)}, 1,1\right), \\ (o(i) o(j))^{\tilde{r}} \frac{\left[r a_{i j}^{s}\right]_{q}}{\tilde{r}\left[d_{i}\right]_{q}} & \text { otherwise, }\end{cases}$ with $a_{i j}^{s}=\max \left\{a_{i j}, a_{j i}\right\}$ (see [Da]).

(ii)-(v) are trivial.

Theorem 12.5. Let $X_{\tilde{n}}^{(k)}$ be different from $A_{1}^{(1)}$ and $A_{2}^{(2)}$. Then $\tilde{\psi}$ induces

$$
\psi=\psi_{X_{\tilde{n}}^{(k)}}: \mathcal{U}_{q}^{\mathrm{Dr}}\left(X_{\tilde{n}}^{(k)}\right) \rightarrow \mathcal{U}_{q}^{\mathrm{DJ}}\left(X_{\tilde{n}}^{(k)}\right) .
$$

Proof. Thanks to Corollary 11.19(iii) and Proposition 12.4(i)-(iii) it is enough to prove that $\tilde{\psi}\left(\mathcal{I}_{0}^{+}(S)\right)=0$; but this is obvious since $\tilde{\psi}\left(\mathcal{I}_{0}^{+}(S)\right)$ is the ideal generated by the ("positive") Serre relations.

In order to prove that $\psi$ is well defined also in the remaining cases we propose two different arguments: a direct one, requiring just some simple commutation relations in $\mathcal{U}_{q}\left(A_{1}^{(1)}\right)$ and $\mathcal{U}_{q}\left(A_{2}^{(2)}\right)$ (see Lemma 12.6); and an argument using the injections $\varphi_{i}$ (see 3.7 ).

Lemma 12.6. In $\mathcal{U}_{q}\left(A_{1}^{(1)}\right)$ we have

(i) $E_{\delta+\alpha_{1}} E_{1}=q^{2} E_{1} E_{\delta+\alpha_{1}}$.

In $\mathcal{U}_{q}\left(A_{2}^{(2)}\right)$ we have:

(ii) $E_{\delta+\alpha_{1}} E_{1}-q^{2} E_{1} E_{\delta+\alpha_{1}}=-[4]_{q} E_{\delta+2 \alpha_{1}}$;

(iii) $E_{\delta+2 \alpha_{1}} E_{1}=q^{4} E_{1} E_{\delta+2 \alpha_{1}}$;

(iv) $q^{-3} E_{\delta+\alpha-1} E_{1}^{2}-\left(q+q^{-1}\right) E_{1} E_{\delta+\alpha-1} E_{1}+q^{3} E_{1}^{2} E_{\delta+\alpha-1}=0$. 
Proof. (i) is an immediate application of the Levendorskii-Soibelman formula (see [LS] and [Da]); for (ii) see [Da]; (iii) is an immediate application of the Levendorskii-Soibelman formula (see [LS] and [Da]); and (iv) follows from (ii) and (iii).

Theorem 12.7. $\tilde{\psi}$ induces $\psi=\psi_{X_{\tilde{n}}^{(k)}}: \mathcal{U}_{q}^{\mathrm{Dr}}\left(X_{\tilde{n}}^{(k)}\right) \rightarrow \mathcal{U}_{q}^{\mathrm{DJ}}\left(X_{\tilde{n}}^{(k)}\right)$.

Proof. Thanks to Corollary 11.19(i)\&(ii), Proposition 12.4 and Theorem 12.5 it is enough to notice that $\tilde{\psi}\left(\mathcal{I}_{0}^{+}(X 1)\right)=0$ in case $A_{1}^{(1)}$ and $\tilde{\psi}\left(\mathcal{I}_{0}^{+}\left(X 3^{1}\right)\right)=0$ in case $A_{2}^{(2)}$, which are immediate consequences of Lemma $12.6(\mathrm{i}) \&(\mathrm{iv})$.

Another proof:

Let $h=1,2, X_{\tilde{n}}^{(k)}=A_{4}^{(2)}, i=\left\{\begin{array}{ll}2 & \text { if } h=1 \\ 1 & \text { if } h=2\end{array}\right.$ and consider the following welldefined diagram:

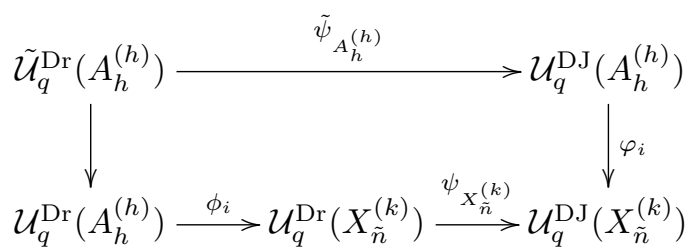

Without loss of generality we can suppose this diagram is commutative, by choos$\operatorname{ing} o_{A_{h}^{(h)}}: 1 \mapsto o_{X_{\tilde{n}}^{(k)}}(i)$.

Then $\tilde{\psi}_{A_{h}^{(h)}}$ factors through $\mathcal{U}_{q}^{\operatorname{Dr}}\left(A_{h}^{(h)}\right)$ (that is, $\psi_{A_{h}^{(h)}}$ is well defined) because $\varphi_{i}$ is injective (see Remark 3.7).

Remark 12.8. (i) $\psi \circ \Omega=\Omega \circ \psi$;

(ii) $\psi \circ t_{i}=T_{\lambda_{i}} \circ \psi$ for all $i \in I_{0}$;

(iii) $\psi \circ \phi_{i}=\varphi_{i} \circ \psi$ for all $i \in I_{0}$;

(iv) $\psi \circ \Theta=\left(\Omega \Xi T_{1}\right) \circ \psi$ in cases $A_{1}^{(1)}$ and $A_{2}^{(2)}$;

(v) $\psi \circ \phi=\varphi$.

Proof. (i)-(iv) follow from Proposition 12.4 and Theorem 12.7, while (v) follows from Remark 11.9 and Theorem 12.7.

Corollary 12.9. $\phi$ is injective.

Proof. This follows from Remark 12.8(v), since $\varphi$ is injective (see Remark 3.7).

We now turn to the surjectivity of $\psi$.

Remark 12.10. By the definition of $\psi$ it is obvious that $E_{i}, F_{i}, K_{i}^{ \pm 1}$ are in the image of $\psi$ for all $i \in I_{0}$. Moreover, since $K_{\delta}^{ \pm 1}$ is in the image of $\psi$, also $K_{0}^{ \pm 1}$ is. But 
by Remark $12.8, \operatorname{Im}(\psi)$ is $\Omega$-stable, so it contains $E_{0}$ if and only if it contains $F_{0}$. Thus it is enough to prove that $E_{0} \in \operatorname{Im}(\psi)$.

In the next theorem it will be used that for $i \in I_{0}, \operatorname{Im}(\psi)$ is a $T_{\lambda_{i}}$-stable subalgebra of $\mathcal{U}_{q}^{\mathrm{DJ}}$ containing $E_{j}, F_{j}, K_{\tilde{j}}^{ \pm 1}\left(j \in I_{0}, \tilde{j} \in I\right)$ (see Remark 12.8); in particular $\mathcal{U}_{q, \pm \alpha}^{\mathrm{DJ}, \pm} \subseteq \operatorname{Im}(\psi)$ for all $\alpha=\sum_{i \in I_{0}} m_{i} \alpha_{i}$.

Theorem 12.11. $\psi: \mathcal{U}_{q}^{\mathrm{Dr}} \rightarrow \mathcal{U}_{q}^{\mathrm{DJ}}$ is surjective.

Proof. Let $\theta=\delta-\alpha_{0}=\sum_{i \in I_{0}} r_{i} \alpha_{i}$. Note that there exists $i \in I_{0}$ such that either $\tilde{d}_{i}=r_{i}=1$ (recall that $\theta$ is a root) or $\tilde{d}_{i}=1, r_{i}=2, a_{i 0} \neq 0$ (so that in particular $\alpha_{0}+\alpha_{i}$ and $\theta-\alpha_{i}$ are roots). Choose such an $i \in I_{0}$ and let $\tilde{\theta}=\theta-\left(r_{i}-1\right) \alpha_{i}$; notice that $\tilde{\theta}$ is a root.

Let $\lambda_{i}=\tau_{i} s_{i_{1}} \cdot \ldots \cdot s_{i_{N}}\left(\right.$ with $\left(l\left(\lambda_{i}\right)=N, \tau_{i} \in \mathcal{T}\right)$; then $\lambda_{i}(\tilde{\theta})=\tilde{\theta}-\delta<0$, so that there exists $h$ such that $s_{i_{N}} \cdot \ldots \cdot s_{i_{h+1}}\left(\alpha_{i_{h}}\right)=\tilde{\theta}$, and we deduce that $f=T_{i_{N}}^{-1} \cdot \ldots \cdot T_{i_{r+1}}^{-1}\left(F_{i_{r}}\right) \in \mathcal{U}_{q,-\tilde{\theta}}^{\mathrm{DJ},-} \subseteq \operatorname{Im}(\psi)$.

Since $\operatorname{Im}(\psi)$ is $T_{\lambda_{i}}$-stable we infer that $T_{\lambda_{i}}(f)=-\tau_{i} T_{i_{1}} \cdot \ldots \cdot T_{i_{r-1}}\left(K_{i_{r}}^{-1} E_{i_{r}}\right)=$ $-K_{\delta-\tilde{\theta}}^{-1} e$ belongs to $\operatorname{Im}(\psi)$, hence so does $e$ with $e \in \mathcal{U}_{q, \delta-\tilde{\theta}}^{\mathrm{DJ},+}$

If $r_{i}=1$ then $\delta-\tilde{\theta}=\alpha_{0}$ and the claim follows $\left(e=E_{0} \in \operatorname{Im}(\psi)\right)$.

If $r_{i}=2$ then $e \in \mathcal{U}_{q, \alpha_{0}+\alpha_{i}}^{\mathrm{DJ},+}$; note that if we are not in case $A_{2 n}^{(2)}$, since $l\left(s_{i} \lambda_{i}\right)=$ $l\left(\lambda_{i}\right)+1$ and $a_{i 0}=-1, T_{i}(e) \in \mathcal{U}_{q, \alpha_{0}}^{\mathrm{DJ},+}$, so that $e=T_{i}^{-1}\left(E_{0}\right)$; on the other hand in case $A_{2 n}^{(2)}$, since $l\left(s_{0} \lambda_{i}\right)=l\left(\lambda_{i}\right)-1$ and $a_{0 i}=-1$, we have $T_{0}^{-1}(e) \in \mathcal{U}_{q, \alpha_{i}}^{\mathrm{DJ},+}$, so that $e=T_{0}\left(E_{i}\right)$. In both cases $e=-\left[E_{0}, E_{i}\right]_{q_{i}^{a}}$. Commuting $e$ with $F_{i}(\in \operatorname{Im}(\psi))$ we get $\operatorname{Im}(\psi) \ni\left[-\left[E_{0}, E_{i}\right]_{q_{i} a_{i 0}}, F_{i}\right]=q_{i}^{a_{i 0}}\left[\left[E_{i}, F_{i}\right], E_{0}\right]_{q_{i}^{-a_{i 0}}}=\left[a_{i 0}\right]_{q_{i}} K_{i} E_{0}$, which concludes the proof.

Proving that $\psi$ is injective requires further analysis

\section{$\S 13$. Appendix: notation}

In this appendix, in order to make it easier for the reader to follow the exposition, most of the notation defined in the paper is collected, with the indication where it is introduced and possibly characterized.

The present list includes neither the notation related to the definition and the structure of the Drinfeld--Jimbo presentation of the quantum algebras, since they are all given synthetically in $\S 3$ where they can be easily consulted, nor the notation introduced in Definition 4.2, because there is no reference to them outside $\S 4$.

Also the relations listed in Proposition 4.25 are not redefined in this appendix, but for some of them other descriptions proposed and used throughout the paper are here recalled. 
Dynkin diagrams, root and weight lattices:

$\tilde{\Gamma}=$ (indecomposable) Dynkin diagram of finite type $\quad \S 1$

$\tilde{I}=$ set of vertices of $\tilde{\Gamma} \quad \S 1$

$\tilde{n}=\# \tilde{I} \quad \S 1$

$\tilde{A}=$ Cartan matrix of $\tilde{\Gamma} \quad \S 1$

$\chi=$ automorphism of $\tilde{\Gamma} \quad \S 1$

$k=o(\chi) \quad \S 1$

$I_{0}=\tilde{I} / \chi \quad \S 1$

$n=\# I_{0}$

${ }^{-}: \quad \tilde{I} \rightarrow I_{0}$ natural projection $\quad \S 1$

$\sim: \quad I_{0} \rightarrow \tilde{I}$ section $\left(a_{i j} \neq 0 \Rightarrow \tilde{a}_{\tilde{i}, \tilde{j}} \neq 0\right) \quad 4.4(4.10)$

$I=I_{0} \cup\{0\} \quad \S 1$

$\Gamma=$ Dynkin diagram of affine type with set of vertices $I \quad \S 1$

$\Gamma_{0}=$ Dynkin subdiagram of $\Gamma$ with set of vertices $I_{0} \quad \S 1$

$A=\left(a_{i j}\right)_{i, j \in I}$ Cartan matrix of $\Gamma \quad \S 1$

$A_{0}=\left(a_{i j}\right)_{i, j \in I_{0}}$ Cartan matrix of $\Gamma_{0} \quad \S 1$

$d_{i}: \quad \min \left\{d_{i} \mid i \in I\right\}=1, \operatorname{diag}\left(d_{i} \mid i \in I\right) A$ symmetric $\quad \S 2$

$\tilde{d}_{i}= \begin{cases}1 & \text { if } k=1 \text { or } X_{\tilde{n}}^{(k)}=A_{2 n}^{(2)} \\ d_{i} & \text { otherwise }\end{cases}$

$\tilde{d}_{i, j}=\max \left\{\tilde{d}_{i}, \tilde{d}_{j}\right\} \quad 4.8$

$\tilde{d}=\max \left\{\tilde{d}_{i} \mid i \in I_{0}\right\} \quad 4.1$

$\tilde{Q}=\mathbb{Z}^{\tilde{I}}=\bigoplus_{i^{\prime} \in \tilde{I}} \mathbb{Z} \tilde{\alpha}_{i^{\prime}}$

$\left(\tilde{\alpha}_{i^{\prime}} \mid \tilde{\alpha}_{j^{\prime}}\right): \quad W$-invariant, $\left(\tilde{\alpha}_{i^{\prime}} \mid \tilde{\alpha}_{i^{\prime}}\right)=2 k / \tilde{d}$ if $\tilde{\alpha}_{i^{\prime}}$ is short $\quad 4.1$

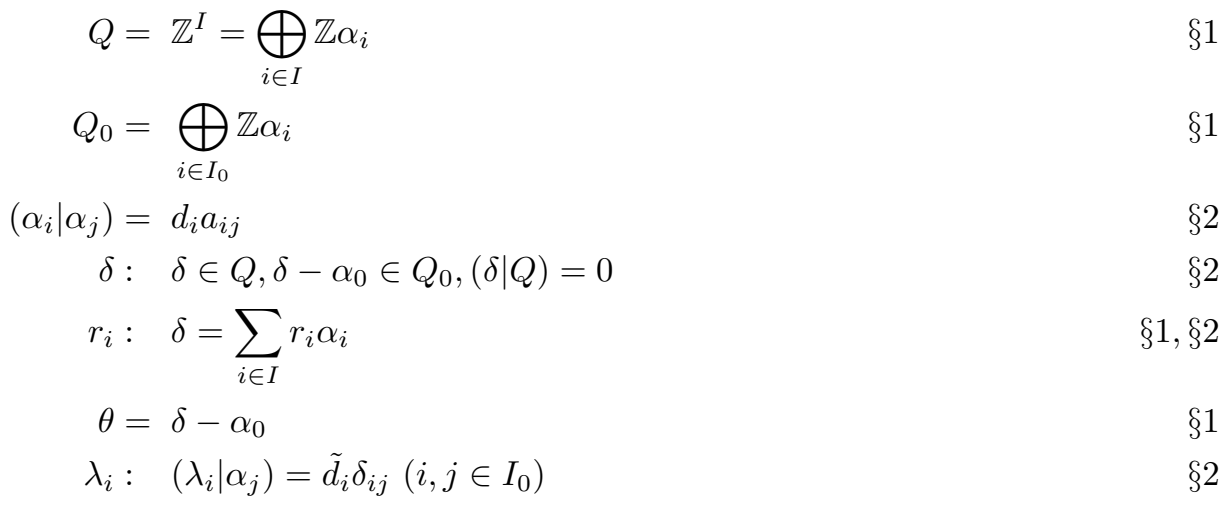




\section{Other notation:}

$$
\begin{aligned}
& \omega=\text { primitive } k^{\text {th }} \text { root of } 1 \\
& q_{i}=q^{d_{i}} \\
& I_{\mathbb{Z}}=\left\{(i, r) \in I_{0} \times \mathbb{Z}\left|\tilde{d}_{i}\right| r\right\} \\
& \varepsilon= \pm 1 \\
& b_{i j r}= \begin{cases}0 & \text { if } \tilde{d}_{i, j} \nmid r \\
{[2 r]_{q}\left(q^{2 r}+(-1)^{r-1}+q^{-2 r}\right) / r} & \text { if }\left(X_{\tilde{n}}^{(k)}, i, j\right)=\left(A_{2 n}^{(2)}, 1,1\right) \quad 4.14 \\
{\left[\tilde{r} a_{i j}\right]_{q_{i}} / \tilde{r}} & \text { otherwise, with } \tilde{r}=r / \tilde{d}_{i, j}\end{cases} \\
& {[a, b]_{u}=a b-u b a} \\
& \mathbb{1}=\mathbb{1}_{l}=(1, \ldots, 1) \in \mathbb{Z}^{l} \\
& \left\{e_{1}, \ldots, e_{l}\right\}=\text { canonical basis of } \mathbb{Z}^{l} \\
& \overline{\left(r_{1}, \ldots, r_{l}\right)}=\left(r_{l}, \ldots, r_{1}\right) \\
& o: \quad I_{0} \rightarrow\{ \pm 1\}, a_{i j} \neq 0 \Rightarrow o(i) o(j)=-1, \\
& \text { if } k \neq 1 \text { and } X_{\tilde{n}}^{(k)} \neq A_{2 n}^{(2)}, a_{i j}=-2 \Rightarrow o(i)=1
\end{aligned}
$$

\section{Generators of $\mathbb{C}(q)$-algebras:}

$$
\begin{aligned}
& \mathcal{G}=\left\{\mathcal{C}^{ \pm 1}, \mathcal{K}_{i^{\prime}}^{ \pm 1}, \mathcal{X}_{i^{\prime}, r}^{ \pm}, \mathcal{H}_{i^{\prime}, s} \mid i^{\prime} \in \tilde{I}, r, s \in \mathbb{Z}, s \neq 0\right\} \\
& G=\left\{C^{ \pm 1}, K_{i}^{ \pm 1}, X_{i, r}^{ \pm}, H_{i, s} \mid i \in I_{0}, r, s \in \mathbb{Z}, s \neq 0\right\} \\
& G^{\prime}=\left\{C^{ \pm 1}, K_{i}^{ \pm 1}, X_{i, r}^{ \pm}, H_{i, s} \mid i \in I_{0}, r, s \in \tilde{d}_{i} \mathbb{Z}, s \neq 0\right\} \\
& \text { 4.6, } 4.26 \\
& \bar{G}=\left\{C^{ \pm 1}, K_{i}^{ \pm 1}, X_{i, r}^{ \pm} \mid i \in I_{0}, r \in \tilde{d}_{i} \mathbb{Z}\right\} \\
& G^{+}=\left\{X_{i, r}^{+} \mid(i, r) \in I_{0} \times \mathbb{Z}\right\} \\
& G^{-}=\left\{X_{i, r}^{-} \mid(i, r) \in I_{0} \times \mathbb{Z}\right\} \\
& G^{\prime,+}=\left\{X_{i, r}^{+} \mid(i, r) \in I_{\mathbb{Z}}\right\} \\
& G^{\prime,-}=\left\{X_{i, r}^{-} \mid(i, r) \in I_{\mathbb{Z}}\right\}
\end{aligned}
$$

Relations in $\mathbb{C}(q)$-algebras:

$$
\begin{aligned}
(\mathcal{D R})= & (\mathcal{Z}, \mathcal{Z} \mathcal{X}, \mathcal{C}, \mathcal{K} \mathcal{K}, \mathcal{K} \mathcal{X}, \mathcal{K} \mathcal{H}, \mathcal{X} \mathcal{X}, \mathcal{H}, \mathcal{H} \mathcal{H} \\
& \left.\mathcal{X} \mathcal{F} \mathcal{G}, \mathcal{X} 3^{\varepsilon}, \mathcal{S}, \mathcal{X P}\right) \\
(D R)= & (Z X, Z H, C U K, C K, K X, K H, X X, H X, H H, \\
& \left.X D, X 1, X 2, X 3^{\varepsilon}, S U L, S 2, S 3\right) \\
& \left(Z X^{ \pm}\right),(Z H)
\end{aligned}
$$




$$
\begin{aligned}
& \left(H X^{ \pm}\right): \quad\left[H_{i, r}, X_{j, s}^{ \pm}\right]= \begin{cases}0 & \text { if } \tilde{d}_{j} \nmid r \\
\pm b_{i j r} C^{\frac{r \mp|r|}{2}} X_{j, r+s}^{ \pm} & \text {if } \tilde{d}_{j} \mid r\end{cases} \\
& \left(X D^{ \pm}\right): \quad M_{(2)}^{ \pm}\left(\left(i, \tilde{d}_{i} r\right),\left(j, \tilde{d}_{j} s\right)\right)=0 \\
& \left(X 1^{ \pm}\right): \quad \sum_{\sigma \in \mathcal{S}_{2}} \sigma \cdot M_{i}^{ \pm}\left(\tilde{d}_{i} r\right)=0 \\
& \left(X 2^{ \pm}\right): \quad \sum_{\sigma \in \mathcal{S}_{2}} \sigma \cdot M_{(2,2)}^{ \pm}(r)=0 \\
& \left(X 3^{\varepsilon, \pm}\right): \quad \sum_{\sigma \in \mathcal{S}_{3}} \sigma \cdot M_{(3)}^{\varepsilon, \pm}(r)=0 \\
& \left(S(U L)^{ \pm}\right): \quad \sum_{\sigma \in \mathcal{S}_{1-a_{i j}}} \sigma \cdot X_{i, j ; 1-a_{i j} ; 1}^{ \pm}(r ; s)=0 \\
& \sum_{\sigma \in \mathcal{S}_{1-a_{i j}}} \sigma \cdot M_{i, j ; 1-a_{i j} ; 1}^{ \pm}(r ; s)=0 \\
& \sum_{\sigma \in \mathcal{S}_{1-a_{i j}}} \sigma \cdot M_{i, j ; 1-a_{i j} ; 1}^{ \pm}\left(\tilde{d}_{i} r ; \tilde{d}_{j} s\right)=0 \\
& \sum_{\sigma \in \mathcal{S}_{1-a_{i j}}} \sigma \cdot X_{i, j ; 1-a_{i j} ; 1}^{ \pm}\left(\tilde{d}_{i} r ; \tilde{d}_{j} s\right)=0 \\
& \left(S 2^{+}\right): \quad \sum_{\sigma \in \mathcal{S}_{2}} \sigma \cdot\left(\left(q^{2}+q^{-2}\right)\left[\left[X_{j, s}^{+}, X_{i, r_{1}+1}^{+}\right]_{q^{2}}, X_{i, r_{2}}^{+}\right]\right. \\
& \left.+q^{2}\left[\left[X_{i, r_{1}+1}^{+}, X_{i, r_{2}}^{+}\right]_{q^{2}}, X_{j, s}^{+}\right]_{q^{-4}}\right)=0 \\
& \sum_{\sigma \in \mathcal{S}_{2}} \sigma \cdot\left(\left(q^{2}+q^{-2}\right)\left[\left[X_{j, s}^{+}, X_{i, r_{1}}^{+}\right]_{q^{-2}}, X_{i, r_{2}+1}^{+}\right]\right. \\
& \left.+\left[X_{j, s}^{+},\left[X_{i, r_{2}+1}^{+}, X_{i, r_{1}}^{+}\right]_{q^{2}}\right]_{q^{-4}}\right)=0 \\
& \sum_{\sigma \in \mathcal{S}_{2}} \sigma \cdot\left(\left[\left[X_{j, s}^{+}, X_{i, r_{1}+1}^{+}\right]_{q^{-2}}, X_{i, r_{2}}^{+}\right]\right. \\
& \left.-q^{2}\left[X_{i, r_{1}+1}^{+},\left[X_{j, s}^{+}, X_{i, r_{2}}^{+}\right]_{q^{-2}}\right]_{q^{-4}}\right)=0 \\
& \left(S 3^{ \pm}\right): \quad \sum_{\sigma \in \mathcal{S}_{2}} \sigma \cdot\left(\left(q^{2}+q^{-4}\right)\left[\left[X_{j, s}^{ \pm}, X_{i, r_{1} \pm 2}^{ \pm}\right]_{q^{3}}, X_{i, r_{2}}^{ \pm}\right]_{q^{-1}}\right. \\
& +\left(1-q^{-2}+q^{-4}\right)\left[\left[X_{j, s}^{ \pm}, X_{i, r_{1} \pm 1}^{ \pm}\right]_{q^{3}}, X_{i, r_{2} \pm 1}^{ \pm}\right]_{q} \\
& \left.+q^{2}\left[\left[X_{i, r_{1} \pm 2}^{ \pm}, X_{i, r_{2}}^{ \pm}\right]_{q^{2}}+\left[X_{i, r_{2} \pm 1}^{ \pm}, X_{i, r_{1} \pm 1}^{ \pm}\right]_{q^{2}}, X_{j, s}^{ \pm}\right]_{q^{-6}}\right)=0 \\
& \left(S k^{ \pm}\right): \quad \sum_{\sigma \in \mathcal{S}_{2}} \sigma \cdot \sum_{\substack{u, v \geq 0 \\
u+v=-1-a_{i j}}} q^{v-u} X_{i, j ; 2 ;-a_{i j}}^{ \pm}\left(r_{1} \pm v, r_{2} \pm u ; s\right)=0 \\
& \sum_{\sigma \in \mathcal{S}_{2}} \sigma \cdot X_{[k]}^{ \pm}(r ; \tilde{d} s)=0
\end{aligned}
$$




$$
\begin{aligned}
\left(T 2^{ \pm}\right): & \sum_{\sigma \in \mathcal{S}_{2}} \sigma \cdot\left[\left[X_{j, s}^{ \pm}, X_{i, r_{1} \pm 1}^{ \pm}\right]_{q^{2}}, X_{i, r_{2}}^{ \pm}\right]=0 \\
& \sum_{\sigma \in \mathcal{S}_{2}} \sigma \cdot M_{[2]}^{ \pm}(r ; 2 s)=0 \\
\left(T 3^{ \pm}\right): & \sum_{\sigma \in \mathcal{S}_{2}} \sigma \cdot\left(\left(q^{2}+1\right)\left[\left[X_{j, s}^{ \pm}, X_{i, r_{1} \pm 2}^{ \pm}\right]_{q^{3}}, X_{i, r_{2}}^{ \pm}\right]_{q^{-1}}\right. \\
& \left.+\left[\left[X_{j, s}^{ \pm}, X_{i, r_{1} \pm 1}^{ \pm}\right]_{q^{3}}, X_{i, r_{2} \pm 1}^{ \pm}\right]_{q}\right)=0 \\
& \sum_{\sigma \in \mathcal{S}_{2}} \sigma \cdot M_{[3]}^{ \pm}(r ; 3 s)=0 \\
\left(H X L^{ \pm}\right): & {\left[H_{i, r}, X_{j, s}^{ \pm}\right]= \pm b_{i j r} C^{\frac{r \mp|r|}{2}} X_{j, r+s}^{ \pm} \quad\left(\tilde{d}_{i} \leq|r| \leq \tilde{d}_{i j}\right) } \\
(X X D): & {\left[X_{i, r}^{+}, X_{j, s}^{-}\right]=0 \quad(i \neq j) } \\
(X X E): & {\left[X_{i, r}^{+}, X_{i,-r}^{-}\right]=\frac{C^{r} k_{i}-C^{-r} k_{i}^{-1}}{q_{i}-q_{i}^{-1}} } \\
\left(X X H^{+}\right): & {\left[X_{i, r}^{+}, X_{i, s}^{-}\right]=\frac{C^{-s} k_{i} \tilde{H}_{i, r+s}^{+}}{q_{i}-q_{i}^{-1}} \quad(r+s>0) } \\
\left(X X H^{-}\right): & {\left[X_{i, r}^{+}, X_{i, s}^{-}\right]=-\frac{C^{-r} \tilde{H}_{i, r+s}^{-} k_{i}^{-1}}{q_{i}-q_{i}^{-1}} \quad(r+s<0) } \\
\mathbb{C}(q) \text {-algebras: } & 5.14 \\
\mathcal{U}_{q}= & \text { Drinfeld and Jimbo quantum algebra } \\
\mathcal{U}_{q}^{\text {DJ }=} & \mathcal{U}_{q}(\Gamma) \\
\mathcal{U}_{q}^{\text {fin }}= & \mathcal{U}_{q}\left(\Gamma_{0}\right) \\
\mathcal{U}_{q}^{\text {Dr }}= & (\mathcal{G} \mid \mathcal{D} \mathcal{R}) \\
\mathcal{U}_{q}^{\text {Dr }}= & (G \mid D R) \\
\tilde{\mathcal{U}}_{q}^{\text {Dr }}= & \left(G \mid Z X^{ \pm}, C U K, C K, K X^{ \pm}, X X, H X L^{ \pm}\right) \\
\overline{\mathcal{U}}_{q}^{\text {Dr }}= & \left(\bar{G} \mid Z X^{ \pm}, C U K, C K\right) \\
\mathcal{F}_{q}^{ \pm}= & \left(G^{ \pm} \mid Z X^{ \pm}\right)=\left(G^{\prime \prime \pm}\right)
\end{aligned}
$$

Elements in $\mathbb{C}(q)$-algebras:

$$
\begin{aligned}
& \tilde{H}_{i, \pm r}^{ \pm}\left(H_{i, r}\right): \sum_{r \in \mathbb{Z}} \tilde{H}_{i, \pm r}^{ \pm} u^{r}=\exp \left( \pm\left(q_{i}-q_{i}^{-1}\right) \sum_{r>0} H_{i, \pm r} u^{r}\right) \\
& \tilde{H}_{i, \pm r}^{ \pm}= \begin{cases}\left(q_{i}-q_{i}^{-1}\right) k_{i}^{-1}\left[X_{i, r}^{+}, X_{i, 0}^{-}\right] & \text {if } r, \pm r>0 \\
\left(q_{i}-q_{i}^{-1}\right)\left[X_{i,-r}^{-}, X_{i, 0}^{+}\right] k_{i} & \text { if } r>0, \pm r<0 \\
1 & \text { if } r=0 \\
0 & \text { if } r<0,\end{cases}
\end{aligned}
$$




$$
\begin{aligned}
& X_{i, j ; l ; a}(r ; s)= \sum_{u=0}^{l}(-1)^{u}\left[\begin{array}{l}
l \\
u
\end{array}\right]_{q_{i}^{a}} X_{i, r_{1}}^{ \pm} \cdot \ldots \cdot X_{i, r_{u}}^{ \pm} X_{j, s}^{ \pm} X_{i, r_{u+1}}^{ \pm} \cdot \ldots \cdot X_{i, r_{l}}^{ \pm} \\
& M_{i, j ; l ; a}(r ; s)= \begin{cases}X_{j, s}^{ \pm} \\
{\left[M_{i, j ; l-1 ; a}^{ \pm}\left(r_{1}, \ldots, r_{l-1} ; s\right), X_{i, r_{l}}^{ \pm}\right]_{q_{i}} a_{i j}-2 a(l-1)} & \text { if } l=0\end{cases} \\
& M_{2}^{ \pm}((i, r),(j, s))=\left[X_{i, r \pm \tilde{d}_{i j}}^{ \pm}, X_{j, s}^{ \pm}\right]_{q_{i} a_{i j}}+\left[X_{j, s \pm \tilde{d}_{i j}}^{ \pm}, X_{i, r}^{ \pm}\right]_{q_{j} a_{j i}} \\
& M_{i}^{ \pm}(r)=\left[X_{i, r_{1} \pm \tilde{d}_{i}}^{ \pm}, X_{i, r_{2}}^{ \pm}\right]_{q_{i}^{2}} \\
& M_{(2,2)}^{ \pm}(r)=\left[X_{1, r_{1} \pm 2}^{ \pm}, X_{1, r_{2}}^{ \pm}\right]_{q^{2}}-q^{4}\left[X_{1, r_{1} \pm 1}^{ \pm}, X_{1, r_{2} \pm 1}^{ \pm}\right]_{q^{-6}} \\
& M_{(3)}^{\varepsilon, \pm}(r)= {\left[\left[X_{1, r_{1} \pm \varepsilon}^{ \pm}, X_{1, r_{2}}^{ \pm}\right]_{q^{2 \varepsilon}}, X_{1, r_{3}}^{ \pm}\right]_{q^{4 \varepsilon}} } \\
& X_{[k]}^{ \pm}(r ; s)=\sum_{u, v \geq 0} q^{v-u} X_{i, j ; 2 ; k}^{ \pm}\left(r_{1} \pm v, r_{2} \pm u ; s\right) \\
& M_{[2]}^{ \pm}(r ; s)= M_{i, j ; 2 ; 1}^{ \pm}\left(r_{1} \pm 1, r_{2} ; s\right) \\
& M_{[3]}^{ \pm}(r ; s)=\left(q^{2}+1\right) M_{i, j ; 2 ; 2}^{ \pm}\left(r_{1} \pm 2, r_{2} ; s\right)+M_{i, j ; 2 ; 1}^{ \pm}\left(r_{1} \pm 1, r_{2} \pm 1 ; s\right) \\
& k_{\alpha}: k_{m \delta+\sum_{i \in I_{0}} m_{i} \alpha_{i}}=C^{m} \prod_{i \in I_{0}} k_{i}^{m_{i}}
\end{aligned}
$$

\section{Relations and ideals:}

(a) given the relations

$$
S_{\zeta}(r, s)=0 \quad\left(\zeta \in \mathcal{Z}, r \in \mathbb{Z}^{l}, s \in \mathbb{Z}^{\tilde{l}}\right)
$$

denote by $\left(R_{\zeta}\right)(\zeta \in \mathcal{Z})$ the relations

$$
S_{\zeta}(r, s)=0 \quad\left(r \in \mathbb{Z}^{l}, s \in \mathbb{Z}^{\tilde{l}}\right) ;
$$

(b) given the relations

$$
S_{\zeta}^{ \pm}(r, s)=0\left(\zeta \in \mathcal{Z}, \quad r \in \mathbb{Z}^{l}, s \in \mathbb{Z}^{\tilde{l}}\right)
$$

denote by $(R)$ the relations

$$
S_{\zeta^{\prime}}(r, s)=0 \quad\left(\zeta^{\prime} \in \mathcal{Z} \times\{ \pm\}, r \in \mathbb{Z}^{l}, s \in \mathbb{Z}^{\tilde{l}}\right)
$$

where $S_{(\zeta, \pm)}=S_{\zeta}^{ \pm}$

(c) given relations $(R)$ as in (a), denote by $\mathcal{I}(R)$ the ideal

$$
\mathcal{I}(R)=\left(S_{\zeta}(r, s) \mid \zeta \in \mathcal{Z}, r \in \mathbb{Z}^{l}, s \in \mathbb{Z}^{\tilde{l}}\right),
$$

by $\mathcal{I}_{\text {const }}(R)$ the ideal

$$
\mathcal{I}_{\text {const }}(R)=\left(S_{\zeta}\left(r \mathbb{1}_{l}, s\right) \mid \zeta \in \mathcal{Z}, r \in \mathbb{Z}, s \in \mathbb{Z}^{\tilde{l}}\right),
$$


by $\mathcal{I}_{0}(R)$ the ideal

$$
\mathcal{I}_{0}(R)=\left(S_{\zeta}(\underline{0}) \mid \zeta \in \mathcal{Z}\right)
$$

and, if $\zeta \in \mathcal{Z}, r \in \mathbb{Z}^{l}, s \in \mathbb{Z}^{\tilde{l}}$, by $\mathcal{I}_{(r, s)}\left(R_{\zeta}\right)$ the ideal

$$
\mathcal{I}_{(r, s)}\left(R_{\zeta}\right)=\left(S_{\zeta}\left(r \mathbb{1}_{l}, s\right)\right)
$$

(d) given relations $\left(R^{ \pm}\right)$as in (a), denote by $\mathcal{I}_{*}^{ \pm}(R)$ the ideals

$$
\mathcal{I}_{*}^{+}(R)=\mathcal{I}_{*}\left(R^{+}\right), \quad \mathcal{I}_{*}^{-}(R)=\mathcal{I}_{*}\left(R^{-}\right) \quad(* \in\{\emptyset, \text { const }, 0\}) ;
$$

(e) given a family of relations $\left({ }^{(h)} R\right)$ as in (a) denote by $\mathcal{I}_{*}\left({ }^{(1)} R, \ldots,{ }^{(m)} R\right)$ the ideals

$$
\mathcal{I}_{*}\left({ }^{(1)} R, \ldots,{ }^{(m)} R\right)=\left(\mathcal{I}_{*}\left({ }^{(1)} R\right), \ldots, \mathcal{I}_{*}\left({ }^{(m)} R\right)\right) \quad(* \in\{\emptyset, \text { const }, 0\}) . \quad 5.1,9.1
$$

\section{(Anti)homomorphisms:}

$$
\begin{array}{rlr}
\bar{\Omega}, \tilde{\Omega}, \Omega: & q \mapsto q^{-1}, C^{ \pm 1} \mapsto C^{\mp 1}, k_{i}^{ \pm 1} \mapsto k_{i}^{\mp 1}, X_{i, r}^{ \pm} \mapsto X_{i,-r}^{\mp} & 6.15,7.6,8.2 \\
\Theta_{\mathcal{F}}^{+}: & q \mapsto q^{-1}, X_{i, r}^{+} \mapsto X_{i,-r}^{+} & 6.15 \\
\Theta_{\mathcal{F}}^{-}: & q \mapsto q^{-1}, X_{i, r}^{-} \mapsto X_{i,-r}^{-} & 6.15 \\
\bar{\Theta}, \tilde{\Theta}, \Theta: & q \mapsto q^{-1}, C^{ \pm 1} \mapsto C^{ \pm 1}, k_{i}^{ \pm 1} \mapsto k_{i}^{\mp 1}, & 6.15,7.13,8.4 \\
& X_{i, r}^{+} \mapsto-X_{i,-r}^{+} k_{i} C^{-r}, X_{i, r}^{-} \mapsto-k_{i}^{-1} C^{-r} X_{i,-r}^{-} & \\
\bar{t}_{i}, \tilde{t}_{i}, t_{i}: & C^{ \pm 1} \mapsto C^{ \pm 1}, k_{j}^{ \pm 1} \mapsto\left(k_{j} C^{-\delta_{i j} \tilde{d}_{i}}\right)^{ \pm 1}, X_{j, r}^{ \pm} \mapsto X_{j, r \mp \delta_{i j} \tilde{d}_{i}}^{ \pm} & 6.15,7.10,8.6 \\
\bar{\phi}_{i}, \tilde{\phi}_{i}, \phi_{i}: & q \mapsto q_{i}, C^{ \pm 1} \mapsto C^{ \pm \tilde{d}_{i}}, k^{ \pm 1} \mapsto k_{i}^{ \pm 1}, X_{r}^{ \pm} \mapsto X_{i, \tilde{d}_{i} r}^{ \pm} & 6.15,7.2 \\
\phi: & K_{i}^{ \pm 1} \mapsto k_{i}^{ \pm 1}, E_{i} \mapsto X_{i, 0}^{+}, F_{i} \mapsto X_{i, 0}^{-}\left(i \in I_{0}\right) & 11.8 \\
\tilde{\psi}, \psi: C^{ \pm 1} \mapsto K_{\delta}^{ \pm 1}, k_{i}^{ \pm 1} \mapsto K_{i}^{ \pm 1}, & 12.3,12.5,12.7 \\
& X_{\tilde{d}_{i} r}^{+} \mapsto o(i)^{r} T_{\lambda_{i}}^{-r}\left(E_{i}\right), X_{\tilde{d}_{i} r}^{-} \mapsto o(i)^{r} T_{\lambda_{i}}^{r}\left(F_{i}\right) &
\end{array}
$$

\section{References}

[A] T. Akasaka, An integral PBW basis of the quantum affine algebra of type $A_{2}^{(2)}$, Publ. RIMS Kyoto Univ. 38 (2002), 803-894. Zbl 1038.17005 MR 1917164

[Be] J. Beck, Braid group action and quantum affine algebras, Comm. Math. Phys. 165 (1994), 555-568. Zbl 0807.17013 MR 1301623

[Bo] N. Bourbaki, Groupes et algèbres de Lie 4, 5, 6, Hermann, Paris, 1968. Zbl 0186.33001 MR 0240238

[CP1] V. Chari and A. Pressley, Quantum affine algebras, Comm. Math. Phys. 142 (1991), 261-283. Zbl 0739.17004 MR 1137064

[CP2] _ Quantum affine algebras and their representations, in Representations of groups (Banff, AB, 1994), CMS Conf. Proc. 16, Amer. Math. Soc., Providence, RI, 1995, 59-78. Zbl 0855.17009 MR 1357195 
[CP3] , Twisted quantum affine algebras, Comm. Math. Phys. 196 (1998), 461-476. Zbl 0915.17013 MR 1645027

[CP4] _ Yangians, integrable quantum systems and Dorey's rule, Comm. Math. Phys. 181 (1996), 265-302. Zbl 0869.17015 MR 1414834

[Da] I. Damiani, The R-matrix for (twisted) affine quantum algebras, in Representations and quantizations (Shanghai, 1998), China High. Educ. Press, Beijing, 2000, 89-144. Zbl 1007.17012 MR 1802170

[Dr1] V. G. Drinfel'd, A new realization of Yangians and of quantum affine algebras, Dokl. Akad. Nauk SSSR 296 (1987), 13-17 (in Russian). Zbl 0667.16004 MR 0914215

[Dr2] Hopf algebras and the quantum Yang-Baxter equation, Dokl. Akad. Nauk SSSR 283 (1985), 1060-1064 (in Russian). Z Zbl 0588.17015 MR 0802128

[FH] E. Frenkel and D. Hernandez, Langlands duality for finite-dimensional representations of quantum affine algebras, Lett. Math. Phys. 96 (2011), 217-261. Zbl 1222.17014 MR 2788912

[GK] O. Gabber and V. G. Kac, On defining relations of certain infinite-dimensional Lie algebras, Bull. Amer. Math. Soc. (N.S.) 5 (1981), 185-189. Zbl 0474.17007 MR 0621889

[H] D. Hernandez, Kirillov-Reshetikhin conjecture: the general case, Int. Math. Res. Notices 2010, 149-193. Zbl pre05665748 MR 2576287

[IM] N. Iwahori and H. Matsumoto, On some Bruhat decomposition and the structure of the Hecke rings of p-adic Chevalley groups, Inst. Hautes Études Sci. Publ. Math. 25 (1965), 5-48. Zbl 0228.20015 MR 0185016

[Jm] M. Jimbo, A $q$-difference analogue of $U(\mathfrak{g})$ and the Yang-Baxter equation, Lett. Math. Phys. 10 (1985), 63-69. Zbl 0587.17004 MR 0797001

[Jn] N. H. Jing, On Drinfeld realization of quantum affine algebras, in The Monster and Lie algebras (Columbus, OH, 1996), Ohio State Univ. Math. Res. Inst. Publ. 7, de Gruyter, Berlin, 1998, 195-206. Zbl 0983.17013 MR 1650669

[JZ1] N. H. Jing and H. L. Zhang, Addendum to 'Drinfeld realization of twisted quantum affine algebras' , Comm. Algebra 38 (2010), 3484-3488. Zbl 1219.17009 MR 2724232

[JZ2] , Drinfeld realization of twisted quantum affine algebras, Comm. Algebra 35 (2007), 3683-3698. Zbl 1168.17012 MR 2362678

[K] V. G. Kac, Infinite dimensional Lie algebras, Birkhäuser, Boston, 1983. Zbl 0537.17001 MR 0739850

[LS] S. Z. Levendorskii and Ya. S. Soibel'man, Some applications of the quantum Weyl groups, J. Geom. Phys. 7 (1990), 241-254. Z Zbl 0729.17009 MR 1120927

[L] G. Lusztig, Finite dimensional Hopf algebras arising from quantized universal enveloping algebras, J. Amer. Math. Soc. 3 (1990), 257-296. Zbl 0695.16006 MR 1013053

[M] H. Matsumoto, Générateurs et relations des groupes de Weyl généralisés, C. R. Acad. Sci. Paris 258 (1964), 3419-3422. Zbl 0128.25202 MR 0183818

[N] H. Nakajima, Quiver varieties and finite-dimensional representations of quantum affine algebras, J. Amer. Math. Soc. 14 (2001), 145-238. Zbl 0981.17016 MR 1808477 